\title{
SEGMENTAÇÃO DOS USUÁRIOS DE CARTÃO DE CRÉDITO POR MEIO DA ANÁLISE DE CESTO DE COMPRAS
}

Dissertação apresentada ao Programa de Pós-Graduação em Administração da Faculdade de Administração e Contabilidade da Universidade de São Paulo, para obtenção do título de mestre em Ciências.

Orientadora: Profa. Dra. Alessandra de Ávila Montini

\section{Versão Corrigida}

(versão original disponível na Faculdade de Economia, Administração e Contabilidade)

São Paulo 
Prof. Dr. João Grandino Rodas

Reitor da Universidade de São Paulo

Prof. Dr. Reinaldo Guerreiro

Diretor da Faculdade de Economia, Administração e Contabilidade

Prof. Dr. Adalberto Américo Fischmann

Chefe do Departamento de Administração

Prof. Dr. Lindolfo Galvão de Albuquerque Coordenador do Programa de Pós-Graduação em Administração 


\section{SEGMENTAÇÃO DOS USUÁRIOS DE CARTÃO DE CRÉDITO POR MEIO DA ANÁLISE DE CESTO DE COMPRAS}

Dissertação apresentada ao Programa de Pós-Graduação em Administração da Faculdade de Administração e Contabilidade da Universidade de São Paulo, para obtenção do título de mestre em Ciências.

Orientadora: Profa. Dra. Alessandra de Ávila Montini

\section{Versão Corrigida}

(versão original disponível na Faculdade de Economia, Administração e Contabilidade)

São Paulo 
FICHA CATALOGRÁFICA

Elaborada pela Seção de Processamento Técnico do SBD/FEA/USP

Tavares, Pedro Daniel

Segmentação dos usuários de cartão de crédito por meio da análise de cesto de compras / Pedro Daniel Tavares. -- São Paulo, 2011.

$145 \mathrm{p}$.

Dissertação (Mestrado) - Universidade de São Paulo, 2012.

Orientadora: Alessandra de Ávila Montini.

1. Segmentação de mercado 2. Cartão de crédito I. Universidade de São Paulo. Faculdade de Economia, Administração e Contabilidade II. Título.

$\mathrm{CDD}-658.802$ 


\section{DEDICATÓRIA}

Dedico à minha família que me apoia incondicionalmente e aceita minha dedicação aos estudos e ao João Pedro, que chegou há pouco, e deu muito mais sentido a tudo. 


\section{AGRADECIMENTOS}

Agradeço a todos que se preocuparam e colaboraram com o desenvolvimento desta pesquisa; à instituição financeira que me proporcionou a pesquisa; aos meus gestores que compreenderam e propiciaram que eu conciliasse os estudos com a atividade profissional; aos mestres que me orientaram e me conduziram na transposição de cada desafio; ao SAS que, por meio do programa Fellowship SAS, possibilitou a realização desta dissertação. 
RESUMO

Esta dissertação de mestrado tem como objetivo, elaborar um modelo de segmentação baseando-se no comportamento comprovado de consumo de clientes, valendo-se das técnicas de Análise de Associação e Análise de Cesto de Compras, aplicadas aos dados das faturas de cartão de crédito dos clientes.

A partir do modelo proposto, testou-se a previsibilidade das próximas transações dos clientes por meio de uma amostra de validação.

A motivação desta pesquisa provém de três pilares: Contexto Científico, Tecnológico e Mercadológico.

No Contexto Científico, apesar de já terem sido publicados artigos que associam a utilização do cartão de crédito a perfis de segmentação de clientes, não se encontram publicados estudos que relacionam dados da própria utilização do cartão como fonte de informação do cliente. A razão mais provável para isso é a dificuldade no levantamento dos dados fundamentais para este tipo de pesquisa. Com o apoio de uma grande instituição financeira, este trabalho está se tornando viável, sob o preceito da análise apenas sobre bases de clientes anônimos e que não transpareça informações estratégicas da instituição.

No contexto tecnológico, com a tecnologia de informação em crescente desenvolvimento, as operações feitas com cartão de crédito tem o processamento on-line em tempo real, promovendo a troca de informação entre o estabelecimento comercial e a instituição emissora do cartão de crédito no momento em que a cobrança é lançada e aceita pelo consumidor final. Isso possibilita que ações promocionais sejam realizadas em toda a cadeia de valor de cartões de crédito, gerando mais valor para os clientes e empresas.

No contexto mercadológico, o Brasil apresentou altas taxas de crescimento do mercado de cartões de crédito nas últimas décadas, substituindo os outros meios mais antigos de pagamento e de crediário. Especialmente no Brasil, observam-se compras pagas com o uso do cartão de crédito parceladas com e sem juros, o que contribui para a substituição de outras formas de crédito.

Como benefício deste trabalho, concluiu-se que a partir do conhecimento do consumo do cliente, pode-se aplicar a análise de cesto de compras para prever as próximas transações dos clientes, a fim de segmentar os clientes para estimulá-los a aderir a uma determinada oferta.

Palavras chave: Segmentação; Customer Relationship Management; Market Basket Analysis; Link Analysis; Cartão de Crédito. 


\begin{abstract}
The objective of this research is elaborating a Segmentation Model based on credit card client's behavior using Link Analysis and Market Basket Analysis techniques. The proposed model was used to testing the predictability of next client transactions through validation sample.
\end{abstract}

Scientific, technological and marketing scenarios are the three motivational pillars of this research.

On scientific context there were published studies that associate credit card use with segmentation profile of customer. However these studies do not establish relationship between data from own clients credit card utilization. One probably reason for this lack analysis into studies is the difficult collect of fundamental data. This research was feasible with the support of a great Brazilian financial group.

On technological context is observed a wide information technology development. Credit cards transactions have on-line processing. This scenario allows exchange information between market and credit card institution at the moment of final client transaction approval. This technology permits that actions be realized along credit card value chain based on transactions that have been made.

On marketing context, during the latest decades, Brazil has shown large growth rates on credit card beyond older ways of payment. In Brazil, is observed a wide utilization of credit cards in installment purchases contributing for the replacement of other ways of credits.

This research conclude that from the knowledge of client consume profile, using the Market Basket Analysis technique, it is possible to get a forecast of purchase transactions with the objective to stimulate the consumer in accept particular offer.

Keywords: Segmentation; Customer Relationship Management; Market Basket Analysis; Link Analysis; Credit Card. 


\section{SUMÁRIO}

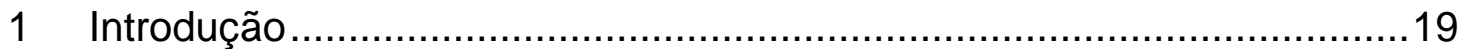

2 Objetivo

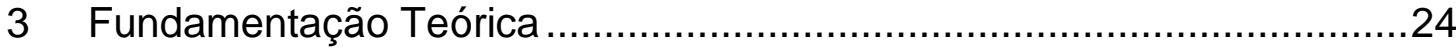

3.1.1 Análise de Associação (AA) …………………………….....24

3.1.2 Análise de Cesto de Compras (ACC) …………......................27

3.1.3 Tecnologia de informação e uso de cartão de crédito ....................28

3.1.4 Tecnologia de informação e a modelagem de grandes bases de dados 29

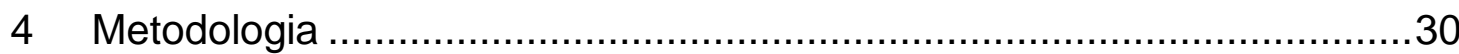

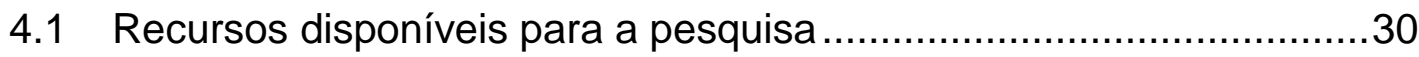

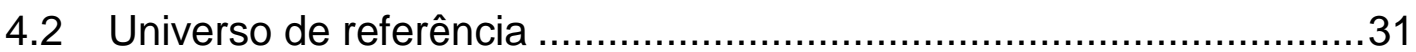

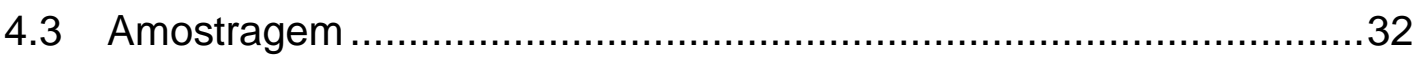

4.3.1 Definição da amostra de clientes ..............................................34

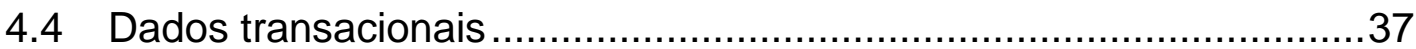

4.4.1 Definição dos produtos analisados.............................................38

4.5 Definição da amostra de validação .................................................40

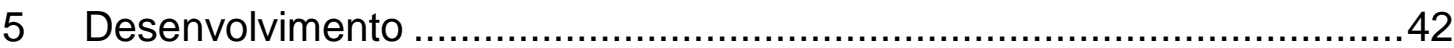

5.1 Análise inicial sobre a base transacional completa ...........................42

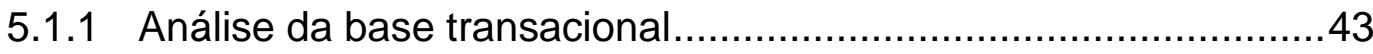

5.2 Aplicação da análise de associação à base de modelagem ...............46

5.2.1 Análise de associação das famílias de produtos nas compras mensais

5.2.2 Análise de associação dos produtos nas compras mensais 54

5.2.3 Análise de associação dos planos de pagamentos nas compras mensais 60

5.2.4 Análise de associação das cidades onde ocorreram as compras mensais 
5.2.5 Análise de associação das famílias de produtos nas compras anuais .69

5.2.6 Análise de associação dos produtos nas compras anuais .75

5.2.7 Análise de associação dos planos de pagamentos nas compras anuais .82

5.2.8 Análise de associação das cidades onde ocorreram as compras anuais .86

5.3 Aplicação da análise de cesto de compras a base de modelagem ....90

5.4 Método de definição das regras para a segmentação de clientes......95

5.5 Segmentação do cliente de cartão de crédito .................................100

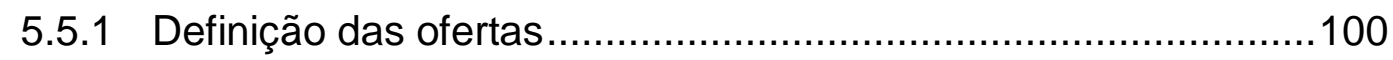

5.5.2 Seleção das regras de associação relevantes ...........................101

5.5.3 Algoritmo de segmentação de clientes (ASC) ...........................101

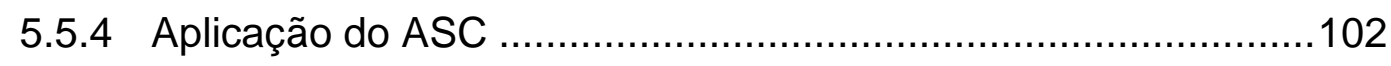

5.5.5 Transações da base de validação............................................104

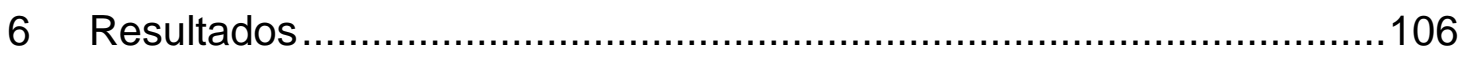

6.1 Eficiência na comunicação .........................................................106

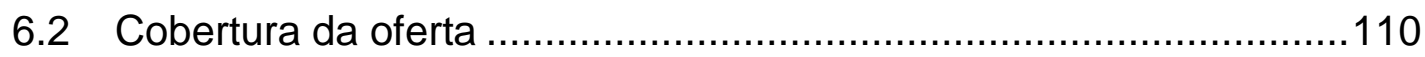

6.3 Equilíbrio entre eficiência e cobertura ………………………......111

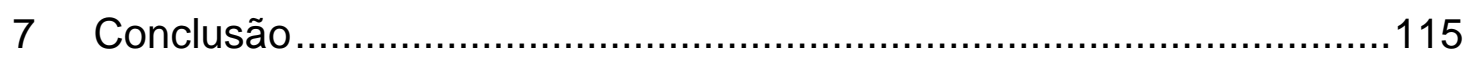

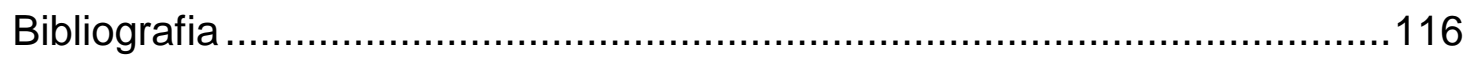

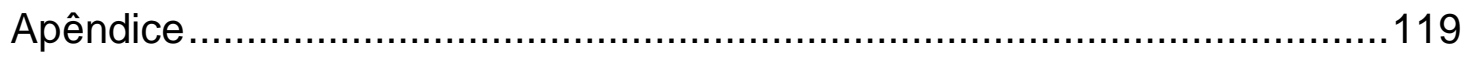




\section{LISTA DE ABREVIATURAS E SIGLAS}
AA - Análise de associações ou Link Analysis
ACC - Análise de cesto de compras ou Market Basket Analysis
ASC - Algoritmo de segmentação de clientes
CO - Região geográfica centro oeste do Brasil
ID - Código numérico identificador de cliente
NE - Região geográfica nordeste do Brasil
NO - Região geográfica norte do Brasil
NO/NE - Macro região geográfica que une norte e nordeste do Brasil
PID - Código numérico identificador de produto
SE - Região geográfica sudeste do Brasil
SE/CO - Macro região geográfica que une sudeste e centro oeste do Brasil
SU - Região geográfica sul do Brasil
UF - Unidade da federação 


\section{LISTA DE SÍMBOLOS}

$\mu_{\mathrm{h}}$ - Saldo médio dos clientes no h-ésimo estrato

A - Transação antecedente

C - Transação consequente

Conf - Confidence

$C v_{h}$ - Coeficiente de variação do saldo no h-ésimo estrato

$D^{2}$ - Relação entre a confiança e a margem de erro

Exp Conf - Expected Confidence

Lift - Lift

n - Quantidade de indivíduos na amostra

N - Quantidade de indivíduos no universo de referência

$\mathrm{N}_{\mathrm{h}}$ - Quantidade de indivíduos no h-ésimo estrato

$\mathrm{n}_{\mathrm{h}}$ - quantidade de indivíduos no h-ésimo estrato da amostra

$\mathrm{P}$ - Probabilidade

s - Desvio padrão da amostra

Sup - Support

$\sigma_{h}$ - Desvio padrão do saldo no h-ésimo estrato

$\sigma_{h}{ }^{2}-$ Variância do saldo no h-ésimo estrato

$\overline{\mathrm{x}}$ - Média da amostra 


\section{LISTA DE TABELAS}

Tabela 1: Dados de identificação dos clientes 31

Tabela 2: Dados das transações

Tabela 3: Universo de clientes e estatísticas descritivas do saldo por região 32

Tabela 4: Tamanho da amostra por região.

Tabela 5: Percentual, média, desvio padrão e coeficiente de variação por macro região

Tabela 6: Percentual, média, desvio padrão e coeficiente de variação por unidade da federação 35

Tabela 7: Percentual, média, desvio padrão e coeficiente de variação por estado civil .36

Tabela 8: Média, desvio padrão e coeficiente de variação por sexo .36

Tabela 9: Média, desvio padrão e coeficiente de variação por ano de início de relacionamento .36

Tabela 10: Média, desvio padrão e coeficiente de variação por década de nascimento do cliente .37

Tabela 11: Percentual de transações por família de produto. .39

Tabela 12: Critério para determinação dos PIDs de acordo com o valor das transações

Tabela 13: Total de transações, frequência relativa percentual, valores médios, medianos, máximos e planos de pagamentos médios, medianos e máximos por família de produtos. .45

Tabela 14: Regras de maior lift - família de produtos por mês. .53

Tabela 15: Regras de maior support - família de produtos por mês .53

Tabela 16: Regras de maior lift - produtos por mês .59

Tabela 17: Regras de maior support - produtos por mês. 60

Tabela 18: Regras de maior lift - planos de pagamento por mês .64

Tabela 19: Regras de maior support - planos de pagamento por mês 64

Tabela 20: Regras de associação do Rio de Janeiro 65

Tabela 21: Regras de maior lift - cidades por mês .69

Tabela 22: Regras de maior support - cidades por mês 69

Tabela 23: Regra 52 de família de produtos por ano .72

Tabela 24: Regras de maior lift - família de produtos por ano .75 
Tabela 25: Regras de maior support - família de produtos por ano .75

Tabela 26: Regras de maior lift - produtos por ano .81

Tabela 27: Regras de maior support - produtos por ano .82

Tabela 28: Regras de maior lift - planos de pagamento por ano .85

Tabela 29: Regras de maior support - planos de pagamento por ano .86

Tabela 30: Regras de maior lift - cidades por ano .90

Tabela 31: Regras de maior support-cidades por ano .90

Tabela 32: 20 primeiras regras ordenadas pelo support lift. .94

Tabela 33: Regras da ACC para o consequente joalheria e joalheria_1 .96

Tabela 34: Regras da AA para famílias de produtos com o consequente joalheria nas compras mensais .96

Tabela 35: Regras da AA para famílias de produtos com o consequente joalheria nas compras anuais

Tabela 36: Regras da AA para produtos com o consequente joalheria nas compras anuais .98

Tabela 37: Ofertas para a determinação dos segmentos de clientes 101

Tabela 38: Clientes por mês de observação na amostra de validação..........103

Tabela 39: Quantidade de clientes por segmento a cada mês observado ...103 Tabela 40: Clientes que realizaram as transações oferecidas.....................104

Tabela 41: Eficiência de comunicação sem segmentação 105

Tabela 42: Clientes que receberam a comunicação e realizaram a transação oferecida. .106

Tabela 43: Eficiência da comunicação com segmentação ........................107

Tabela 44: Redução de custo relativo com a segmentação ........................108

Tabela 45: Cobertura das ofertas ....................................................110

Tabela 46: Resumo dos resultados da aplicação do ASC .........................113

Tabela 47: Regras de associação da ACC utilizadas na segmentação.........129 Tabela 48: Regras de associação das AAs utilizadas na segmentação dos clientes. 


\section{LISTA DE GRÁFICOS}

Gráfico 1: Percentual de transações por família de produtos .43

Gráfico 2: Support versus confidence para as cem primeiras regras de associação das famílias de produtos por mês. .50

Gráfico 3: Lift, confidence, expected confidence e support de família de produtos por mês .51

Gráfico 4: Support versus confidence para as cem primeiras regras de associação de produtos por mês .57

Gráfico 5: Lift, confidence, expected confidence e support de produtos por mês .58

Gráfico 6: Support versus confidence para as cem primeiras regras de associação de planos de pagamentos por mês 62

Gráfico 7: Lift, confidence, expected confidence e support de planos de pagamentos por mês 63

Gráfico 8: Support versus confidence para as primeiras regras de associação de cidades por mês

Gráfico 9: Lift, confidence, expected confidence e support de cidades por mês

Gráfico 10: Support versus confidence para as cem primeiras regras de associação de famílias de produtos por ano. .73 Gráfico 11: Lift, confidence, expected confidence e support de famílias de produtos por ano .73

Gráfico 12: Support versus confidence para as cem primeiras regras de associação de produtos por ano 79

Gráfico 13: Lift, confidence, expected confidence e support de produtos por ano. .80

Gráfico 14: Support versus confidence para as cem primeiras regras de associação de planos de pagamentos por ano.

Gráfico 15: Lift, confidence, expected confidence e support de planos de pagamentos por ano

Gráfico 16: Support versus confidence para as cem primeiras regras de associação de cidades por ano .88 
Gráfico 17: Lift, confidence, expected confidence e support de cidades por ano 88

Gráfico 18: Análise de cesto de compras - 6.537 regras ordenadas por lift...92 Gráfico 19: Dispersão das regras em relação ao support e confidence .93 Gráfico 20: Redução de custo de comunicação relativo com segmentação.108 Gráfico 21: Ganho de eficiência de comunicação com segmentação 109 Gráfico 22: Cobertura da oferta segmentada 111 


\section{LISTA DE ILUSTRAÇÕES}

Figura 1: Representação do universo de transações por ramo de atividade ..25

Figura 2: Grafo do exemplo de análise de associação .27

Figura 3: Modelo de negócio de cartões de crédito .28

Figura 4: Faturas e transações dos clientes da amostra .38

Figura 5: Clientes, faturas e transações sem a amostra de validação 41

Figura 6: Utilização do cartão no período de estudo 42

Figura 7: Box-Plot do valor das transações por família de produto .44

Figura 8: Box-Plot do plano de pagamento por família de produto 44

Figura 9: Recorrência média de compras por família de produtos .46

Figura 10: Grafo de associações de famílias de produtos por mês 49

Figura 11: Matriz de regras de associação de família de produtos por mês...52

Figura 12: Grafo de associações de produtos por mês (parte 1) .55

Figura 13: Grafo de associações de produtos por mês (parte 2) 56

Figura 14: Matriz de regras de associação de produtos por mês .58

Figura 15: Grafo de associações de planos de pagamento por mês 61

Figura 16: Matriz de regras de associação de planos de pagamentos por mês

Figura 17: Grafo de associações das cidades por mês .66

Figura 18: Matriz de regras de associação de cidades por mês .68

Figura 19: Grafo de associações de famílias de produtos por ano .71

Figura 20: Matriz de regras de associação de família de produtos por ano....74

Figura 21: Grafo de associações de produtos por ano (parte1) .77

Figura 22: Grafo de associações de produtos por ano (parte 2) .78

Figura 23: Matriz de regras de associação de produtos por ano .80

Figura 24: Grafo de associações de planos de pagamento por ano .83

Figura 25: Matriz de regras de associação de planos de pagamentos por ano

Figura 26: Grafo de associações de cidades por ano .87

Figura 27: Matriz de regras de associação de cidades por ano. .89

Figura 28: Exemplo da taxonomia dos produtos. 


\section{INTRODUÇÃO}

A segmentação é fundamental para que uma empresa consiga se comunicar com seu público de forma eficiente. A formação e o tratamento de grupos homogêneos de clientes possibilita a oferta de produtos e serviços adequados às demandas dos consumidores e, como resultado do melhor atendimento, a fidelização e o valor percebido dos produtos e serviços aumentam.

A fidelização proporcionada por ações dirigidas aos clientes pode tornar a empresa mais eficiente, por meio da redução dos custos de aquisição e, em consequência, conquistar maior rentabilidade para os acionistas ou proprietários. Os benefícios da retenção dos clientes frente ao custo de aquisição de novos são substancialmente mensuráveis.

É sabido que obter novos clientes custa de três a cinco vezes mais do que manter os clientes existentes (HOFFMAN, BATESON, et al., 2009).

O tratamento individual pode ser considerado o limite da segmentação de clientes, no entanto, o que se pretende com a segmentação eficiente é discriminar grupos de clientes semelhantes. Assim, torna-se viável a customização da comunicação de produtos e serviços, de forma a impressionar os clientes com abordagens dirigidas de modo aparentemente individualizadas, mantendo custos relativamente baixos (BLACKWELL, MINIARD e ENGEL, 2002). Entretanto, aparentemente não é comum que as empresas mantenham cadastros atualizados capazes de refletir e reverberar a realidade de seus clientes a todo momento, bem como de sinalizar seu valor para a organização e seus hábitos.

Uma das filosofias básicas que guiam a abordagem da empresa ao mercado está no território do marketing de segmentos, aqui definido pela decisão de identificar os diferentes grupos constitutivos do mercado e desenvolver produtos específicos para atingir um público selecionado. Esta abordagem é considerada suficientemente útil para localizar as oportunidades de mercado e desenvolver compostos de marketing mais efetivos (KOTLER, 2000). 
O modelo tradicional de segmentação é baseado em atributos demográficos, atitudinais e psicográficos dos clientes e, desde os artigos pioneiros no assunto, a segmentação de clientes é vista como uma forma valiosa de reconhecimento dos atributos do mercado e ganho de eficiência na oferta de produtos para segmentos de mercados específicos.

A diferenciação e a segmentação do mercado são altamente relacionadas (SMITH, 1956). Mas a noção de que os segmentos podem ser discriminados a partir das atitudes dos clientes surgiu em 1974 com uma pesquisa de escolhas de atributos de produtos. A partir destas escolhas foi possível verificar alta correlação com a demografia dos clientes pesquisados, idade e renda (BLATTBERG e SEN, 1974).

Duas importantes publicações de 1978 testaram modelos e algoritmos de segmentação de clientes. Estes estudos seguiram a premissa de que, frente a mercados heterogêneos, a segmentação de clientes eleva a rentabilidade esperada dos produtos (WIND, 1978), e que a percepção das indústrias sobre as preferências dos clientes relativas a atributos de produtos de uso corriqueiros são informações preciosas para o posicionamento das empresas com seus clientes (BLATTBERG, BUESING, et al., 1978).

Um estudo de segmentação, que acompanhou o hábito de consumo dos clientes de um determinado produto alimentício por 78 semanas, conseguiu diferenciar os clientes fiéis a uma mesma marca dos clientes que não se mantiveram fiéis. Entre estes dois grupos de clientes, houve diferenças significativas quanto à sensibilidade a preços (KAMAKURA e RUSSEL, 1989), mas até então os estudos mostraram que a segmentação tem um grande potencial enquanto ferramenta para incrementar os negócios, com uma aplicação muito específica e simplificada.

As publicações recentes demonstram uma tendência à preocupação da aplicação das técnicas de segmentação para encontrar os clientes mais rentáveis da base de clientes das organizações a partir da modelagem do life time value (KIM, JUNG, et al., 2006), mas estas publicações não demonstram a aplicação destas técnicas nas companhias. Há possibilidade de exploração de meios alternativos de 
segmentação com o objetivo de agrupar os clientes a partir de dados facilmente coletáveis (LEE e PARK, 2005).

Alguns estudos já foram realizados por meio da associação da forma de utilização do cartão às características dos portadores, como é o caso do artigo "Credit Cards as Lifestyle Facilitator", que tratou de uma pesquisa que associou a utilização do cartão de crédito com o estilo de vida de cada um dos 28 americanos utilizadores de cartão de crédito observados.

Este artigo mostrou apenas que a frequência do uso do cartão de crédito pode discriminar o estilo de vida do usuário. A partir deste dado, é possível classificar grupos de clientes quanto ao risco de crédito e quanto ao relacionamento com a instituição financeira, além de propiciar a utilização destes agrupamentos na prática de fidelizar os clientes existentes (BERNTHAL, CROCKETT e ROSE, 2005).

O consumidor de cartão de crédito também foi estudado de acordo com fatores demográficos, demonstrando a previsibilidade na intensidade de utilização de acordo com o sexo, idade e renda (GARCIA, 1980).

Outra característica do comportamento de consumo por meio do cartão foi descrita no artigo "Time-Inconsistent Preferences and Consumer Self-Control" que relacionou a utilização do cartão como meio de obtenção de crédito em contraposição com a função de meio de pagamento. O comportamento de uso do cartão foi utilizado para prever a ocorrência de inadimplência neste estudo $(\mathrm{HOCH}$ e LOEWENSTEIN, 1991).

Já no artigo, "Differences in spending habits and credit use of college students" foi apresentada a modelagem de utilização de cartão de crédito baseado em informações obtidas por meio de questionários aplicados a estudantes universitários americanos (HAYHOE, LEACH, et al., 2000).

As informações tratadas pela indústria de cartões de crédito podem trazer um excelente modelo de segmentação de clientes, a partir de dados constantemente atualizados. Esta afirmação encontra respaldo na existência de bases de dados 
reais, suficientemente detalhadas a respeito do consumo diário de todos os clientes que fazem uso do cartão de crédito. A todo momento, as redes de cartões de crédito atualizam suas bases com os valores gastos pelos clientes, as lojas que tem visitado em cidades ou países por onde estão a circular no momento da aquisição dos bens.

Desta forma, a motivação para o desenvolvimento deste trabalho deve-se à busca da compreensão e aplicação dos métodos que explicam o agrupamento de indivíduos, a partir da observação do histórico de consumo. Procurou-se a utilização das técnicas estatísticas de AA (Análise de Associações) e ACC (Análise de Cesto de Compra) na obtenção da segmentação dos clientes de cartões de crédito. 


\section{OBJETIVO}

Esta dissertação tem como objetivo propor a elaboração e aplicação de um modelo de segmentação de clientes de cartão de crédito a partir da apuração de informações transacionais de consumo dos indivíduos estudados, por meio da aplicação das técnicas de Análise de Cesto de Compras (ACC) e Análise de Associações (AA) às bases.

O modelo de segmentação deve identificar, a partir das transações dos clientes, ofertas a serem comunicadas de forma eficiente, buscando incrementar o valor percebido dos produtos e serviços oferecidos aos clientes, associando a assertividade da oferta para o cliente com baixos custos de comunicação para a indústria de cartões de crédito. 


\section{FUNDAMENTAÇÃO TEÓRICA}

O agrupamento de indivíduos com características semelhantes possibilita a elaboração de modelos com o objetivo de projetar ações de uma grande quantidade de indivíduos. As técnicas de Análise de Associação (AA) e Análise de Cesto de Compras (ACC) serão aplicadas a dados históricos com o objetivo de projetar futuras ações.

\subsubsection{Análise de Associação (AA)}

A teoria da AA é construída sobre o campo matemático da teoria dos grafos e tem por finalidade detectar padrões de associação em um conjunto de ações (LINOFF e BERRY, 2004).

Para o desenvolvimento deste trabalho, as ações consideradas foram as compras realizadas e pagas por meio do cartão de crédito.

Os padrões de associações foram traduzidos em termos de regras, cujas análises da incidência de ações consequentes são dadas pelas ações antecedentes.

Ações antecedentes também são descritas na literatura como Left Hand Rule e ações consequentes são descritas como Right Hand Rule.

A Figura 1 representa o universo de transações realizadas pelos indivíduos observados, analisados do ponto de vista do ramo de atividade, cujas compras foram realizadas. 


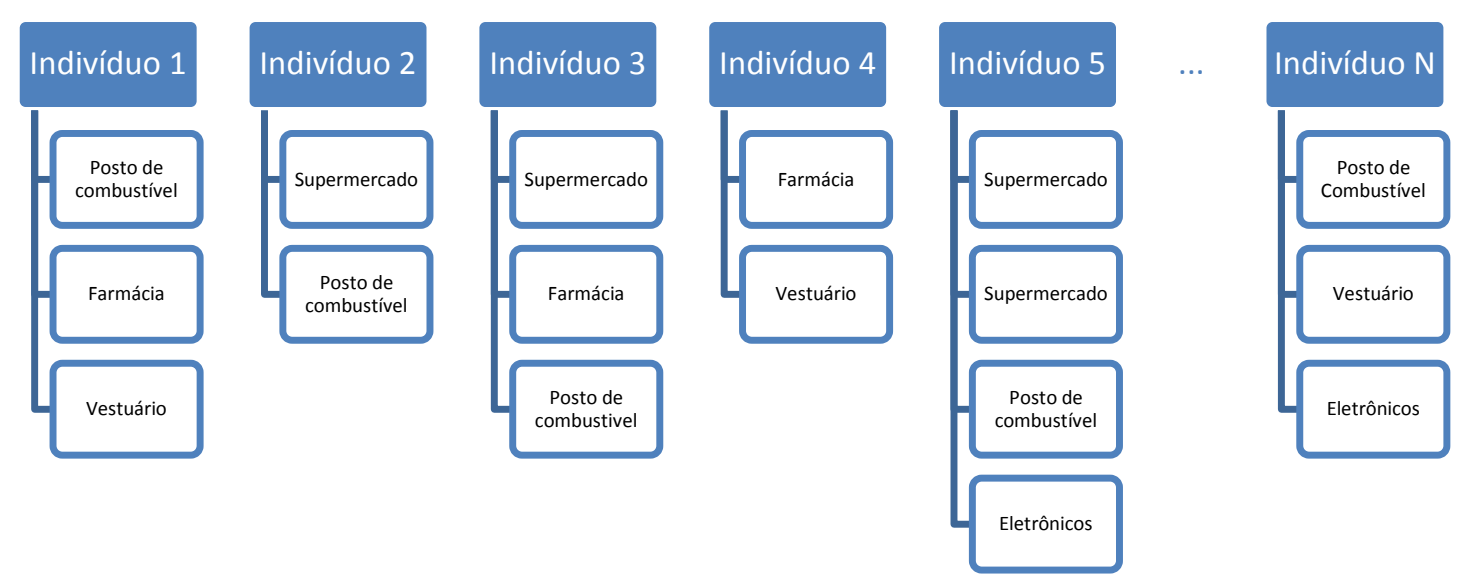

Figura 1: Representação do universo de transações por ramo de atividade

A AA utiliza quatro medidas básicas para determinar as regras de associações. Estas medidas são denominadas: support, confidence, expected confidence e lift.

Para a determinação das medidas básicas da $A A$, são levadas em consideração as informações sumarizadas das ações, em termos de antecedentes e consequentes, realizadas por todos os clientes.

Considere uma ação antecedente $(\mathrm{A})$ que provoca uma ação consequente (C).

O support é definido como a probabilidade de ocorrer $\mathrm{A}$ e $\mathrm{C}$ e é dado por:

$$
\text { Sup }=P(A \cap C) .
$$

O confidence, que está apresentado na Equação 2, representa a probabilidade de ocorrer uma transação consequente $(C)$, dado que houve uma transação antecedente $(A)$.

$$
\text { Conf }=P(C / A) \text {. }
$$

A expressão pode ser reescrita como indicado na Equação 3.

$$
P(C / A)=\frac{P(C \cap A)}{P(A)}
$$


O expected confidence, que está representado na Equação 4 trata-se da probabilidade de ocorrer a ação consequente independente das demais ações.

$$
\operatorname{Exp} \operatorname{Conf}=P(C) .
$$

O lift, representado na Equação 5 mede a verossimilhança da regra, ou seja, mede o aumento das chances do cliente realizar a compra consequente, dado que realizou a antecedente.

$$
\text { Lift }=\frac{\text { Conf }}{\text { Exp Conf }}=\frac{P(C / A)}{P(C)}=\frac{P(A \cap C)}{P(C) \cdot P(A)}
$$

A associação de fato existe quando o lift é diferente de um, ou seja, quanto maior o lift, mais positiva será a relação, caracterizando atração entre as ações. Quando o lift é inferior a um, configura-se a repulsão entre as ações.

A Figura 2 é um exemplo de representação em formato de grafo para a relação entre alguns eventos. Nela, as circunferências representam os ramos de atividade onde transações são realizadas. $O$ tamanho e a cor da circunferência demonstram a diferenciação das mais para as menos volumosas. As maiores e vermelhas são mais numerosas do que as menores e azuis. As setas que relacionam as circunferências representam a intensidade da associação entre as circunferências. As setas largas e vermelhas representam uma associação forte, de confidence elevado enquanto as azuis e estreitas, representam as associações de menor confidence. A cor roxa representa um valor de medida de associação entre o vermelho e o azul.

Como exemplo na figura, interpreta-se que as transações em supermercado são mais numerosas do que as dos demais ramos de atividade. Há uma forte associação entre as compras em farmácias, antecedentes a compras em supermercados. Compras em postos de combustível e eletrônicos são antecedentes de compras em supermercado, porém com uma intensidade de associação inferior à associação com farmácia e, finalmente vestuário e supermercado possuem 
associações mútuas, ou seja, ambos podem ser antecedentes ou consequentes do outro.

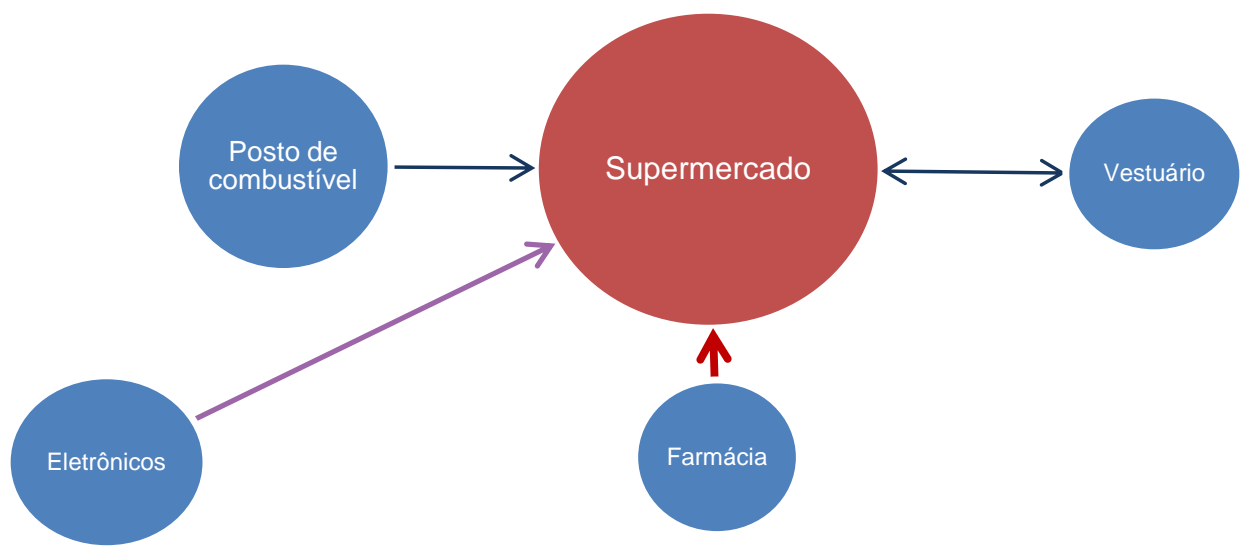

Figura 2: Grafo do exemplo de análise de associação

\subsubsection{Análise de Cesto de Compras (ACC)}

A ACC procura identificar as relações, a partir da AA, com o incremento da taxonomia ou classificação das transações em famílias.

Esta técnica de análise se demonstra aplicável ao problema de identificação de regras de associação entre as transações no cartão de crédito e indica as regras de associação das transações que formam o cesto de compras, com base nas medidas de support e confidence, de forma análoga a AA (BRIN, MOTWANI, et al., 1997).

Para a realização da ACC, são necessários três níveis de informações (LINOFF e BERRY, 2004):
a. Clientes;
b. Compras (sequência de compras);
c. Itens (classificados em famílias).

A análise de cesto de compras demanda bases transacionais grandes, para que as relações demonstrem-se com clareza. A grande dimensão das bases de estudo, torna imperativo o uso de algoritmos de reconhecimento das regras de 
associação de rápida execução e que comporte grandes quantidades de combinações (AGRAWAL e SRIKANT, 1994).

\subsubsection{Tecnologia de informação e uso de cartão de crédito}

Com a tecnologia de informação em pleno desenvolvimento, as operações feitas com cartão de crédito têm quase que em sua totalidade processamento em tempo real, ou seja, a troca de informação entre o estabelecimento comercial e a instituição emissora do cartão de crédito acontece no momento em que a cobrança é lançada e aceita pelo consumidor final (portador do cartão de crédito).

Esta troca de informação, atualmente se limita a uma positivação (aceitação) da cobrança por parte do portador do cartão. Convém destacar que outras informações - ofertas de produtos; convites a visitar outros estabelecimentos; quantas vezes o cliente realizou compras no mesmo estabelecimento - podem ser trocadas durante esta conexão entre o estabelecimento comercial e a instituição emissora do cartão.

O modelo de negócio do cartão de crédito nacional é composto por três instituições: o cliente, o lojista e a bandeira, sendo que os bancos fazem a intermediação com os clientes portadores de cartões bem como com os lojistas conforme ilustrado na Figura 3.

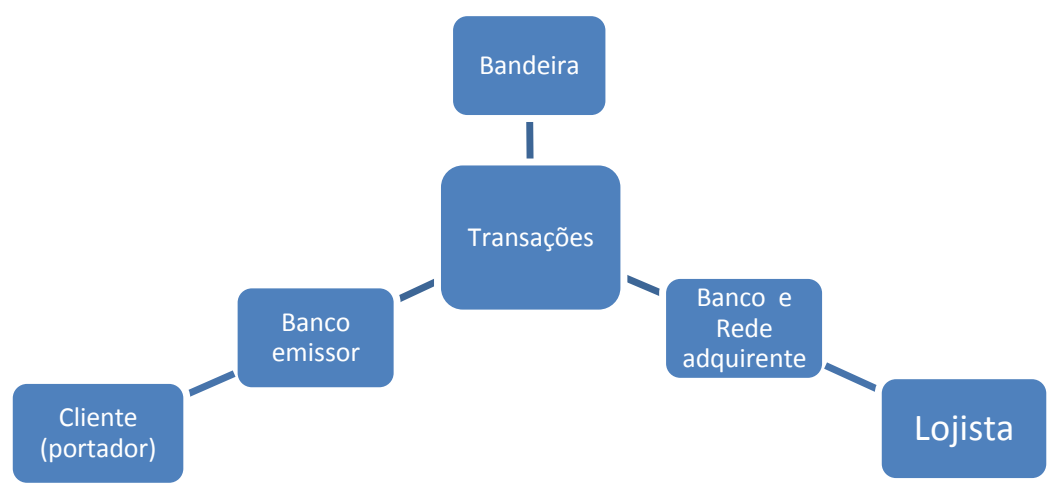

Figura 3: Modelo de negócio de cartões de crédito

Observando a Figura 3, a partir de uma transação, o fluxo da informação ocorre da seguinte maneira: 
O cliente apresenta o cartão a um lojista que, por meio de uma conexão telefônica ou por internet da rede adquirente, envia a informação da venda para a bandeira, que repassa a informação ao banco emissor do cartão. $O$ banco emissor do cartão verifica instantaneamente a condição de crédito do cliente e devolve a resposta à bandeira e, através da rede adquirente valida a operação junto ao lojista, emitindo uma autorização de pagamento.

\subsubsection{Tecnologia de informação e a modelagem de grandes bases de dados}

Com o fluxo de informações descrito no item anterior, os bancos adquirentes, bandeira e bancos emissores acumulam as informações de transações por cliente (ou por cartão) e a partir destas informações podem entender quais são as associações fortes ou fracas entre os tipos de transações realizadas pelos portadores de cartão de crédito.

Uma ferramenta estatística amplamente utilizada pelas instituições financeiras é o software SAS, um importante sistema para Business Intelligence. Este sistema é fundamental para a extração, manipulação e análise de bases de dados extensas de cartão de crédito. 


\section{METODOLOGIA}

\subsection{Recursos disponíveis para a pesquisa}

O levantamento das informações necessárias ao estudo é altamente controlado pela legislação de sigilo bancário e esse controle não facilita a execução deste tipo de pesquisa. No entanto, com o apoio de uma grande instituição do sistema financeiro brasileiro, esta pesquisa pode ser viabilizada, seguindo preceitos rigorosos do sigilo das informações de qualquer cliente, bem como da própria instituição.

Para tanto, foi elaborado um plano de análise em que os clientes são anônimos, diferenciados entre si a partir de um código aleatório. Desta forma, se mantém preservado o sigilo dos dados dos clientes utilizados na pesquisa e também a consistência das informações pesquisadas.

O conjunto de clientes analisado não é o total de clientes, mas uma amostra parcial de clientes desta instituição. As informações estratégicas da instituição financeira e do negócio utilizado na pesquisa se mantêm preservados.

Para a extração e manipulação dos dados, fez-se o uso do software SAS Enterprise Guide V.4.1 e o SAS Enterprise Miner V. 5.2, cuja licença pertence à mesma instituição financeira fornecedora das informações. Todo o tratamento dos dados até a geração das bases com identificadores aleatórios foi realizado de acordo com o sistema protegido da instituição financeira.

Para que fosse possível a utilização dos programas nas análises apresentadas nesta dissertação, o SAS, por meio de um programa de incentivo a pesquisa, denominado Fellowship SAS, forneceu licenças temporárias para a instalação em uma máquina. 


\subsection{Universo de referência}

A base analisada possui 652.749 clientes usuários de cartão de crédito que haviam contratado seus cartões de crédito até 30 dias antes do início das observações. Não é possível mencionarmos de qual instituição ou qual bandeira de cartões de crédito trata-se, para manter o sigilo das informações da instituição financeira que propiciou este estudo.

Como dados iniciais desta base, extraiu-se os dados cadastrais dos clientes, bem como a utilização inicial (saldo acumulado que traziam do período anterior ao primeiro mês do acompanhamento). Na Tabela 1 apresenta-se a disposição dos dados da base de clientes.

\begin{tabular}{ll}
\hline \multicolumn{1}{c}{ Descrição do Campo } & \multicolumn{1}{c}{ Tipo } \\
\hline Código do cliente & Numérico (9) \\
Região geográfica & Texto (2) \\
Cidade de cadastro & Texto (25) \\
Estado civil & Texto (25) \\
Sexo & Texto (10) \\
Data de cadastro & Data \\
Década de nascimento & Número (2) \\
Utilização inicial & Numérico (9) \\
\hline Tabela 1: Dados de identificação dos clientes &
\end{tabular}

Para a realização da pesquisa, a base de clientes foi observada entre janeiro e dezembro de 2009. Ao final dos 12 meses observou-se mais de 16 milhões de transações realizadas pelos 652.749 clientes.

Na Tabela 2 apresenta-se a disposição dos dados da base de transações.

\begin{tabular}{ll}
\hline \multicolumn{1}{c}{ Descrição do Campo } & \multicolumn{1}{c}{ Tipo } \\
\hline Código da transação & Numérico (9) \\
Código do cliente & Numérico (9) \\
Data da transação & Data \\
Valor da transação & Numérico (9) \\
Plano de pagamento & Numérico (2) \\
Ramo de atividade do ponto de venda & Texto (25) \\
Família de produto & Texto (16) \\
Cidade do ponto de venda & Texto (25) \\
\hline
\end{tabular}

Tabela 2: Dados das transações 
A Tabela 3 apresenta, em cada macro região, o total de clientes $\left(\mathrm{N}_{\mathrm{h}}\right)$, média da utilização inicial $\left(\mu_{h}\right)$, desvio padrão $\left(\sigma_{h}\right)$, variância $\left(\sigma_{h}{ }^{2}\right)$ e coeficiente de variação $\left(\mathrm{Cv}_{\mathrm{h}}\right)$.

O termo macro região é relacionado às regiões do Brasil. As regiões norte e nordeste são denotadas NO/NE, sudeste e centro oeste denotadas SE/CO e a região sul denotada SU.

O termo saldo corresponde ao valor da fatura que o cliente possuía antes da primeira observação (em dezembro de 2008), ou seja, este saldo representa o saldo inicial, antes da primeira utilização a ser contemplada pelo estudo.

\begin{tabular}{lccccc}
\hline Macro Região & $\mathrm{N}_{h}$ & $\mu_{h}$ & $\sigma_{h}$ & $\sigma_{h}{ }^{2}$ & $C v_{h}$ \\
\hline NO/NE & 225.945 & 308,68 & 403,17 & 162.554 & 131 \\
SE/CO & 253.800 & 119,30 & 265,20 & 70.330 & 222 \\
S & 173.004 & 149,82 & 252,00 & 63.505 & 168 \\
\hline
\end{tabular}

Tabela 3: Universo de clientes e estatísticas descritivas do saldo por região

Nota-se que a macro região NO/NE apresenta o maior saldo médio e o menor coeficiente de variação, enquanto que a macro região SE/CO apresenta o menor saldo médio e o maior coeficiente de variação.

Mesmo com toda a capacidade que o software de business intelligence nos oferece, o tratamento de mais de 16 milhões de transações exigiria uma capacidade de processamento muito elevada. Optou-se por extrair uma amostra da base de dados e aplicar as técnicas de associação à amostra selecionada.

\subsection{Amostragem}

Para gerar uma amostra de informações, já que os dados transacionais de clientes são muito volumosos, optou-se por trabalhar com uma amostra estratificada que represente este universo de 652.749 clientes. Os estratos definidos são as macro regiões geográficas dos clientes, demonstradas anteriormente na Tabela 3. 
A amostragem foi realizada por meio da técnica de amostragem por alocação de Neyman para a média.

O tamanho da amostra obtido com base na extração de uma amostra por meio da alocação de Neyman para a média é dada por:

$$
n=\frac{\left(\sum N_{h} \cdot \sigma_{h}\right)^{2}}{N^{2} \cdot D^{2}+\sum N_{h} \cdot \sigma_{h}^{2}}
$$

$\mathrm{Na}$ Equação 6, $\mathrm{n}$ corresponde à quantidade de clientes da amostra, $\mathrm{Nh}$ corresponde à quantidade de clientes no h-ésimo estrato da população, oh corresponde ao desvio padrão da utilização inicial no h-ésimo estrato da população, $N$ corresponde à quantidade de clientes da população, $\sigma_{h}{ }^{2}$ corresponde à variância do saldo no h-ésimo estrato da população, $\mathrm{D}^{2}=\frac{d^{2}}{z^{2}}, d=R \$ 3,00$ (margem de erro), $z=1,96$ ( $z$ é obtido por meio da distribuição normal padrão para uma confiança de $95 \%)$

O tamanho da amostra, para cada estrato $\left(n_{h}\right)$, obtido com base na extração de uma amostra pela alocação de Neyman para a média, é dado por:

$$
n_{h}=\frac{N_{h} \cdot \sigma_{h}}{\sum_{h}\left(N_{h} \cdot \sigma_{h}\right)} . n .
$$

Observa-se na Expressão 7, que o valor $n_{h}$ é proporcional a $N_{h} \sigma_{h}$. Por este motivo, a técnica de alocação de Neyman se torna mais adequada para a amostragem em questão, pois tal proporcionalidade faz com que a amostra reflita de forma mais clara a diferença entre as variâncias dos estratos.

Considerando a confiança de $95 \%$ e uma margem de erro de 3\%, obtém-se por meio da Expressão 6 e das informações apresentadas na Tabela 3, uma amostra de 38.357 clientes. 


\subsubsection{Definição da amostra de clientes}

Após a obtenção do tamanho total da amostra obtém-se, por meio da Expressão 7, o tamanho da amostra para cada região geográfica (ver Tabela 4).

\begin{tabular}{lc}
\hline Região & $\mathrm{n}_{\mathrm{h}}$ \\
\hline $\mathrm{NO} / \mathrm{NE}$ & 17.298 \\
$\mathrm{SE} / \mathrm{CO}$ & 12.781 \\
$\mathrm{~S}$ & 8.278 \\
\hline \multicolumn{2}{l}{ Tabela 4: Tamanho da amostra por região }
\end{tabular}

As Tabelas 5, 6, 7, 89 e 10 apresentam as características cadastrais da amostra de 38.357 clientes no momento inicial do estudo - com os respectivos percentuais, saldos médios $(\mu)$, desvios padrão $(\sigma)$ e coeficientes de variação $(C v)$ para cada categoria - a fim de representar, com clareza, as informações iniciais da amostra.

A média de utilização inicial da amostra é de $\mathrm{R} \$ 212,84$, com desvio padrão de $R \$ 339,99$, resultando em um coeficiente de variação de 160 .

Ao interpretar a Tabela 5 em comparação com a Tabela 3, percebe-se que a técnica demonstra-se eficiente, visto que as médias e os desvios padrão apresentam, na amostra e no universo de referência, valores próximos.

\begin{tabular}{lcccc}
\hline Macro região & Percentual & $\bar{x}$ & $\mathrm{~s}$ & $\mathrm{Cv}$ \\
\hline $\mathrm{N} / \mathrm{NE}$ & $45,1 \%$ & 311,42 & 398,35 & 128 \\
$\mathrm{SE} / \mathrm{CO}$ & $33,3 \%$ & 116,85 & 253,37 & 217 \\
$\mathrm{~S}$ & $21,6 \%$ & 155,05 & 258,94 & 167 \\
\hline $\begin{array}{l}\text { Tabela 5: Percentual, } \\
\text { macro região }\end{array}$
\end{tabular}

A Tabela 6 apresenta as medidas descritivas por unidade da federação. Para a clara distinção da concentração, esta tabela está ordenada do maior percentual para o menor. Observa-se que $63,67 \%$ dos clientes estão concentrados em cinco estados. 


\begin{tabular}{|c|c|c|c|c|}
\hline UF & Percentual & $\bar{x}$ & $\mathrm{~s}$ & $\mathrm{Cv}$ \\
\hline São Paulo & $22,65 \%$ & 124,29 & 247,40 & 199 \\
\hline Pernambuco & $12,41 \%$ & 350,64 & 417,90 & 119 \\
\hline Bahia & $11,20 \%$ & 305,86 & 386,93 & 127 \\
\hline Rio Grande do Sul & $10,67 \%$ & 161,21 & 254,18 & 158 \\
\hline Paraná & $6,74 \%$ & 152,55 & 283,00 & 186 \\
\hline Rio Grande do Norte & $4,52 \%$ & 296,34 & 373,20 & 126 \\
\hline Paraíba & $4,39 \%$ & 297,37 & 398,02 & 134 \\
\hline Santa Catarina & $4,17 \%$ & 143,35 & 228,21 & 159 \\
\hline Rio de Janeiro & $3,94 \%$ & 103,24 & 268,99 & 261 \\
\hline Ceará & $3,79 \%$ & 254,32 & 373,40 & 147 \\
\hline Alagoas & $3,16 \%$ & 361,03 & 407,91 & 113 \\
\hline Minas Gerais & $3,11 \%$ & 78,13 & 213,16 & 273 \\
\hline Maranhão & $2,50 \%$ & 272,40 & 405,75 & 149 \\
\hline Sergipe & $1,56 \%$ & 349,35 & 415,92 & 119 \\
\hline Piauí & $1,48 \%$ & 187,84 & 345,65 & 184 \\
\hline Distrito Federal & $1,12 \%$ & 145,67 & 330,66 & 227 \\
\hline Espírito Santo & $1,09 \%$ & 112,61 & 270,58 & 240 \\
\hline Goiás & $0,85 \%$ & 111,80 & 347,88 & 311 \\
\hline Mato Grosso do Sul & $0,53 \%$ & 84,10 & 155,55 & 185 \\
\hline Pará & $0,04 \%$ & 91,84 & 148,75 & 162 \\
\hline Mato Grosso & $0,02 \%$ & 103,52 & 167,08 & 161 \\
\hline Tocantins & $0,01 \%$ & 125,50 & 278,97 & 222 \\
\hline Amapá & $0,01 \%$ & 209,84 & 276,36 & 132 \\
\hline Rondônia & $0,01 \%$ & 109,57 & 160,23 & 146 \\
\hline Roraima & $0,01 \%$ & 400,86 & 518,22 & 129 \\
\hline Amazonas & $0,01 \%$ & 194,15 & 336,28 & 173 \\
\hline
\end{tabular}

Percebe-se na análise da Tabela 7, que apresenta as medidas descritivas por estado civil, pouca diferença entre os clientes de diferentes estados civis. Contudo o estado civil que apresenta maior saldo médio é o dos viúvos, com média de $R \$$ 235,44 . 


\begin{tabular}{lcccc}
\hline Estado Civil & Percentual & $\bar{x}$ & $\mathrm{~s}$ & Cv \\
\hline Casado & $50,8 \%$ & 211,28 & 346,20 & 164 \\
Solteiro & $34,2 \%$ & 214,04 & 330,08 & 154 \\
Viúvo & $5,9 \%$ & 235,44 & 361,05 & 153 \\
Divorciado & $5,0 \%$ & 214,35 & 357,03 & 167 \\
Outro & $4,3 \%$ & 188,95 & 287,88 & 152 \\
\hline
\end{tabular}

Tabela 7: Percentual, média, desvio padrão e coeficiente de variação por estado civil

A Tabela 8 apresenta as medidas descritivas por sexo. Nota-se que as mulheres apresentam a utilização inicial ligeiramente superior. Contudo, o coeficiente de variação dos homens é também pouco superior, indicando uma maior dispersão entre as médias de utilização dos homens frente a das mulheres.

\begin{tabular}{llccc}
\hline Sexo & Percentual & $\bar{x}$ & $\mathrm{~s}$ & Cv \\
\hline Feminino & $51,3 \%$ & 219,12 & 338,41 & 154 \\
Masculino & $48,7 \%$ & 206,23 & 341,53 & 166 \\
\hline
\end{tabular}

Tabela 8: Média, desvio padrão e coeficiente de variação por sexo

A Tabela 9 apresenta as medidas descritivas por início de relacionamento. Percebe-se que quanto mais antigo o relacionamento do cliente com o cartão, maior se apresenta a média da utilização inicial do cliente e que quanto mais recente o relacionamento, maior o coeficiente de variação.

\begin{tabular}{llccc}
\hline Início de relacionamento & Percentual & $\bar{x}$ & $\mathrm{~s}$ & $\mathrm{Cv}$ \\
\hline Até 2001 & $9,4 \%$ & 460,65 & 530,25 & 115 \\
2002 a 2004 & $15,8 \%$ & 352,76 & 424,6 & 120 \\
2005 a 2006 & $29,7 \%$ & 176,97 & 288,54 & 163 \\
2007 & $21,4 \%$ & 156,87 & 256,73 & 164 \\
2008 a 2009 & $23,6 \%$ & 115,81 & 200,59 & 173
\end{tabular}

Tabela 9: Média, desvio padrão e coeficiente de variação por ano de início de relacionamento

A Tabela 10 apresenta as medidas descritivas por década de nascimento dos clientes. Percebe-se que a maior utilização inicial média está entre os clientes nascidos na década de 60 . 


\begin{tabular}{llccc}
\hline Década de nascimento & Percentual & $\bar{x}$ & $\mathrm{~s}$ & $\mathrm{Cv}$ \\
\hline Até 50s & $34,4 \%$ & 218,56 & 361,47 & 165 \\
60 s & $22,9 \%$ & 236,82 & 366,15 & 155 \\
70 s & $26,2 \%$ & 220,21 & 332,34 & 151 \\
80s e após & $16,5 \%$ & 155,87 & 249,96 & 160
\end{tabular}

Tabela 10: Média, desvio padrão e coeficiente de variação por década de nascimento do cliente

\subsection{Dados transacionais}

Após a extração da amostra de clientes, agrupou-se à base de cadastro dos clientes as transações realizadas durante o período de 12 meses.

Cada cliente da amostra pode ter até 12 faturas mensais agrupando transações ocorridas dentro de um mesmo mês.

Para agrupar os clientes às suas transações, foi utilizada uma chave de identificação (ID), bem como a data de cada transação foi utilizada para agrupar as faturas mensais.

Como a análise foi feita sobre uma amostra da base de clientes, existe a possibilidade de clientes desta base terem usado o cartão em todos os meses do ano, mas também é possível que parte destes clientes tenha um comportamento de paralisação de sua utilização no meio do período, ou mesmo tenham passado os 12 meses sem sequer utilizar uma vez o cartão. Os clientes que não fizeram uso do cartão no período observado não serão considerados na modelagem.

A Figura 4 ilustra esta distribuição de clientes em relação a suas faturas e transações no período analisado. 


\section{Clientes na amostra 38.357}

Clientes com pelo menos uma Fatura 27.222

214.584 faturas 984.359 transações

Figura 4: Faturas e transações dos clientes da amostra

\subsubsection{Definição dos produtos analisados}

Para a aplicação das técnicas de AA e ACC, é necessária a definição dos produtos a serem analisados. Para tanto, a partir dos dados transacionais descritos na Tabela 2, criou-se uma nova coluna com um código numérico identificador de produto, denominada PID.

O PID possui três componentes básicos (informações oriundas de cada transação): plano de pagamento, família de produto e valor da transação.

Como definições, para o reconhecimento dos produtos e seus componentes, tem-se:

a) planos de pagamento correspondem à quantidade de parcelas em que a transação será paga pelo cliente. Pode-se ter a partir de uma prestação (compra à vista) até 24 prestações;

b) famílias de produto correspondem a dezessete agrupamentos de ramos de atividades diferentes de empresas que comercializam produtos e serviços;

A Tabela 11 apresenta as famílias de produtos, ordenadas pela frequência percentual na amostra de 984.359 transações realizadas nos 12 meses. 


\begin{tabular}{lc}
\hline Família de Produto & Frequência \\
\hline Supermercado & $24 \%$ \\
Vestuário & $15 \%$ \\
Lojas em geral & $11 \%$ \\
Farmácia & $10 \%$ \\
Postos de gasolina & $5 \%$ \\
Construção & $5 \%$ \\
Telecomunicações / Eletrônicos & $4 \%$ \\
Diversão & $4 \%$ \\
Veículos & $4 \%$ \\
Serviços & $3 \%$ \\
Alimentos & $3 \%$ \\
Perfumaria & $3 \%$ \\
Geral & $2 \%$ \\
Joalherias & $2 \%$ \\
Livraria & $2 \%$ \\
Móveis & $2 \%$ \\
Viagens & $1 \%$ \\
\hline Tabela 11: Percentual de transações por família de produto
\end{tabular}

c) valor da transação corresponde ao valor de cada compra realizada pelo cliente portador do cartão de crédito.

Para a definição da dimensão do valor do produto ao PID, avaliou-se que, para cada combinação entre família de produto e plano de pagamento, a distribuição dos valores das transações demonstram grandes variações. Concluiu-se, então, que valores fixos de faixas de valor não expressariam diferenças entre os produtos de famílias e planos de pagamentos diferentes.

Como solução para esta definição, determinou-se, 7 faixas de valor, encontradas a partir da utilização dos valores dos 3 quartis e dos desvios padrão dos valores das transações, para cada combinação entre família de produto e plano de pagamento, conforme a Tabela 12.

Para esta base de dados analisada, os desvios padrão do valor das transações superam a diferença entre o segundo e o terceiro quartil para todas as famílias de produtos e planos de pagamento. Por isso foi possível a determinação dos produtos conforme a Tabela 12. Recomenda-se, para trabalhos futuros utilizar apenas as medidas interquartis para determinar as faixas de preço. 


\begin{tabular}{cl}
\hline Faixa & Critério de faixa de valor de transação \\
\hline 1 & Até o valor do primeiro quartil das transações \\
2 & Do primeiro ao segundo quartil das transações \\
3 & Do segundo ao terceiro quartil das transações \\
4 & Do terceiro quartil até a mediana +1 desvio padrão das transações \\
5 & Da mediana +1 desvio padrão até a mediana +2 desvios padrão das transações \\
6 & Da mediana +2 desvios padrão até a mediana +3 desvios padrão das transações \\
7 & Valores superiores à mediana +3 desvios padrão das transações \\
\hline
\end{tabular}

Tabela 12: Critério para determinação dos PIDs de acordo com o valor das transações

A partir das informações: plano de pagamento, família de produto e valor da transação, discriminou-se 1.358 produtos diferentes, segundo a programação demonstrada no Anexo 1.

Cada PID tem uma descrição de conformidade com a seguinte regra: Família de produto _ Plano de pagamento _ Faixa de valor.

Para um maior esclarecimento, tomam-se dois exemplos de produtos:

Alimentos_1_F01:0|--12 - produto da família de produtos alimentos, pago em uma prestação (à vista), com valor de até $\mathrm{R} \$ 12,00$ - faixa 1 (até o valor do primeiro quartil).

Joalherias_6_F02:200|--298 - produto da família de produtos joalheria, pago em seis prestações, está na segunda faixa de valores de $R \$ 200,00$ até $R \$ 298,00$ (entre o primeiro e segundo quartil).

\subsection{Definição da amostra de validação}

Para a validação do modelo de segmentação proposto, reservou-se $20 \%$ das faturas extraídas na amostra. Esta separação foi feita de forma aleatória entre as 214.584 faturas dos 27.222 clientes que utilizaram o cartão no período analisado.

Sendo assim, reservou-se 42.917 faturas como não participantes da modelagem da segmentação transacional dos clientes de cartão de crédito. 
A Figura 5 retoma o conceito da base inicial do estudo e ilustra novamente a distribuição de clientes em relação a suas faturas no período analisado, já subtraídas as faturas reservadas para a amostra de validação. Extraída a amostra de validação, a base a ser utilizada na modelagem apresenta 26.767 clientes que utilizaram seus cartões em média 29,4 vezes, durante 6,4 meses do ano estudado, resultando em 4,6 utilizações por mês.

\section{Clientes na amostra 38.357}

Clientes com pelo menos uma Fatura 26.767

171.667 faturas 787.986 transações

Figura 5: Clientes, faturas e transações sem a amostra de validação 


\section{DESENVOLVIMENTO}

\subsection{Análise inicial sobre a base transacional completa}

Como ponto de partida das análises, considera-se a Figura 4 da Seção 4. Cumpre observar que dos 38.357 clientes da amostra estudada, 11.135 não tiveram qualquer uso dos seus cartões no período estudado; ou seja, embora contassem com o meio de pagamento e crédito disponíveis por meio destes cartões, utilizaram outros meios de pagamentos para realizar as suas compras.

É importante salientar que por representar a base de clientes, a amostra contém: a) clientes que fizeram uso do cartão durante todo período observado; b) clientes que iniciaram o seu uso no período estudado; c) clientes que pararam de utilizar no período estudado; d) clientes que iniciaram o uso e pararam de utilizar nestes 12 meses de estudo; e) clientes que embora com a possibilidade de utilização do cartão, não a fizeram durante o período observado (estes clientes, conforme dito anteriormente, não contribuirão com a modelagem).

A Figura 6 demonstra a classificação dos clientes da amostra a partir do ponto de vista da utilização do cartão nos 12 meses de estudo. Verifica-se que a maioria dos clientes não teve a utilização dos seus cartões durante todos os meses observados.

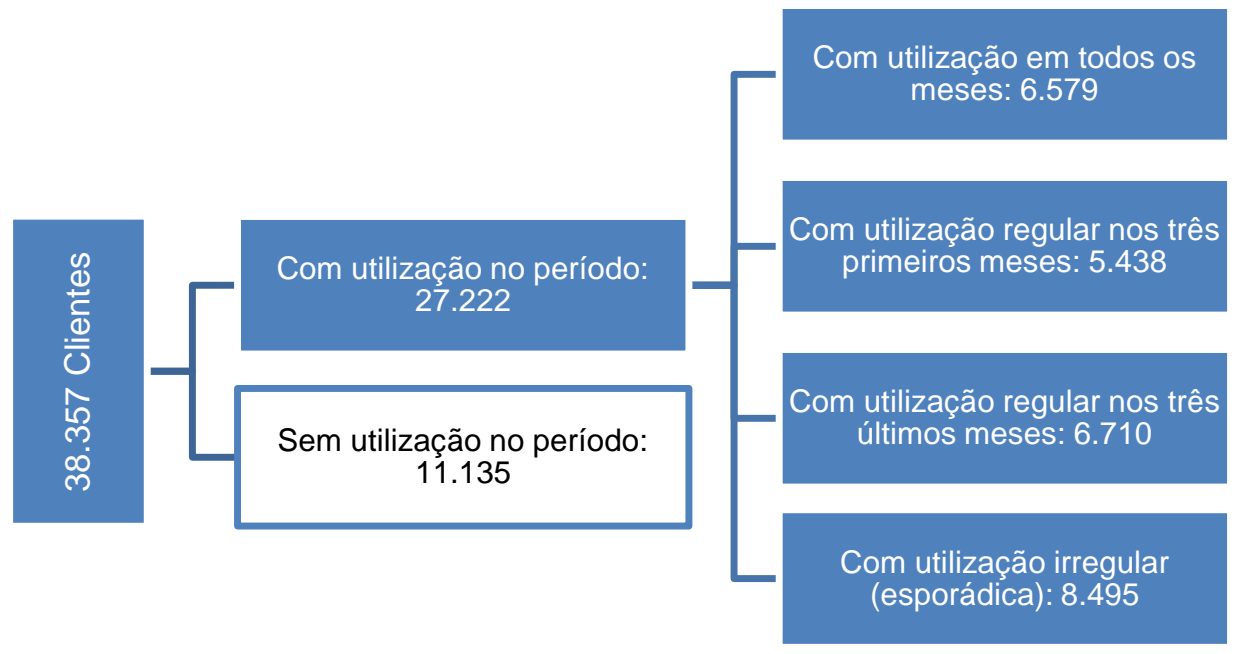

Figura 6: Utilização do cartão no período de estudo 
Considera-se regular, a utilização em três meses consecutivos no início ou no final do período.

Estes clientes com utilização irregular, não tiveram utilização sequencial no início ou no final do período, mas fizeram algum uso no do período. Desse modo, seus dados foram observados na modelagem da utilização.

Em resumo, a base estudada tem 27.222 clientes que utilizaram seus cartões em média 36,2 vezes, durante 7,9 meses do ano estudado, resultando em 4,6 utilizações por mês.

\subsubsection{Análise da base transacional}

O Gráfico 1 apresenta a distribuição percentual das 787.986 transações por família de produto. A família de produtos que mais se destaca, com $34 \%$ das transações é a supermercado, depois, entre $11 \%$ e 10\% tem-se vestuário, posto de gasolina e farmácia.

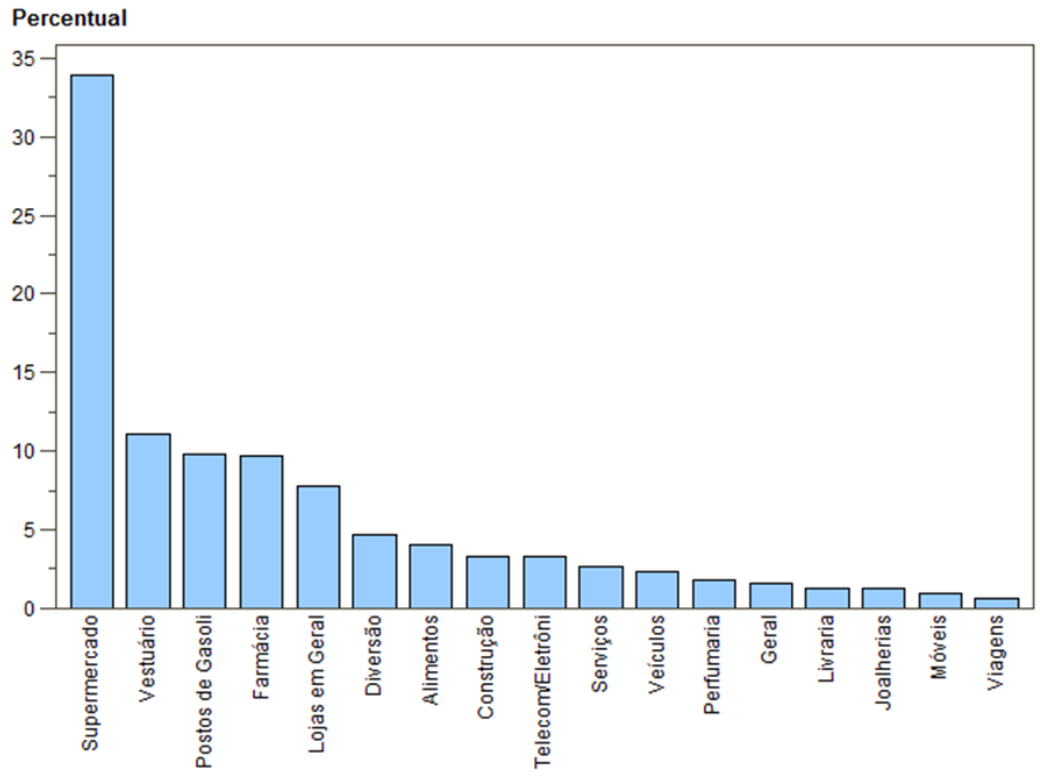

Gráfico 1: Percentual de transações por família de produtos

A Figura 7 apresenta o a dispersão dos valores das transações por família de produto. Cada família de produtos está representada por um box-plot. Nota- 
se que as famílias de produtos supermercado e lojas em geral apresentam muitos outliers.

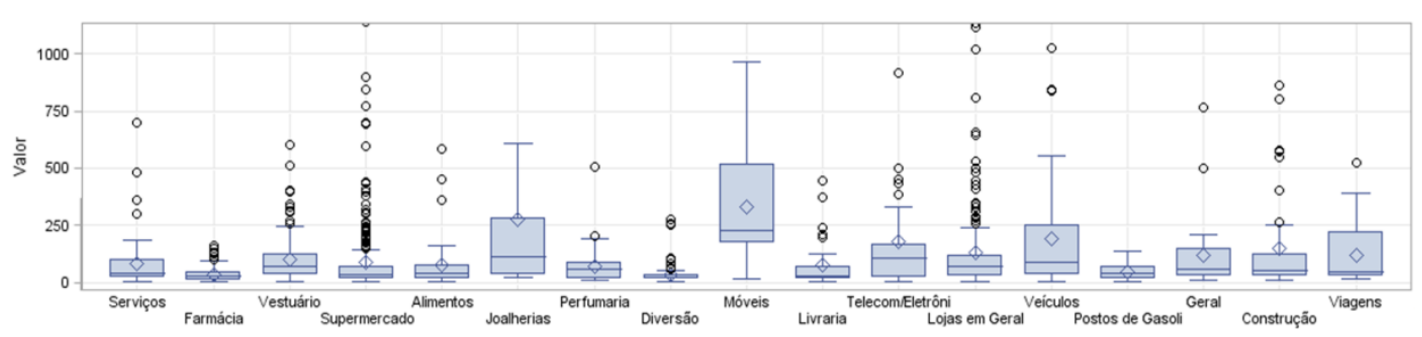

Figura 7: Box-Plot do valor das transações por família de produto

A Figura 8 apresenta a dispersão da quantidade de parcelas das transações observadas por família de produtos. Nota-se que os gastos em alimentos, diversão e posto de gasolina são pagos em poucas parcelas.

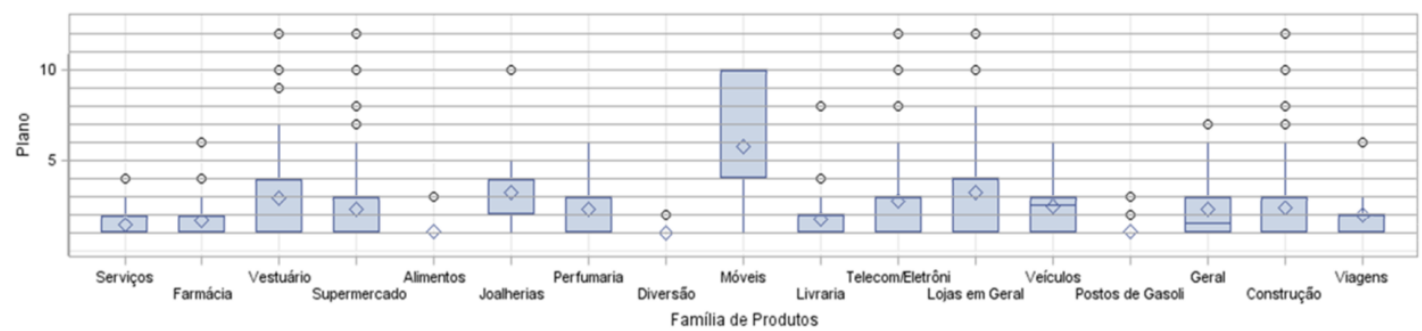

Figura 8: Box-Plot do plano de pagamento por família de produto

A Tabela 13 apresenta o total $(N)$ e a frequência relativa percentual das transações; os valores de transação médios, medianos, máximos e desvio padrão para cada família de produtos; os valores de plano de pagamento médios, medianos, máximos e desvio padrão. Percebe-se a concentração de transações nas famílias de produtos supermercado, vestuário, posto de gasolina e farmácia. A família de produtos móveis se destaca como a de maior valor médio por transação, e mais longo plano de pagamento médio. 


\begin{tabular}{|c|c|c|c|c|c|c|c|c|c|c|}
\hline & \multicolumn{2}{|c|}{ TRANSAÇÕES } & \multicolumn{4}{|c|}{ VALOR } & \multicolumn{4}{|c|}{ PLANO DE PAGAMENTO } \\
\hline & $\mathrm{N}$ & $\%$ & Média & Mediana & Máximo & $\begin{array}{l}\text { Desvio } \\
\text { Padrão }\end{array}$ & Média & Mediana & Máximo & $\begin{array}{l}\text { Desvio } \\
\text { Padrão }\end{array}$ \\
\hline Alimentos & 31.711 & 4,02 & 40 & 22 & 2.270 & 66 & 1,04 & 1 & 10 & 0,24 \\
\hline Construção & 26.071 & 3,31 & 151 & 61 & 6.845 & 256 & 2,26 & 1 & 12 & 1,91 \\
\hline Diversão & 37.181 & 4,72 & 29 & 20 & 3.956 & 45 & 1,04 & 1 & 10 & 0,35 \\
\hline Farmácia & 76.157 & 9,66 & 37 & 25 & 2.500 & 43 & 1,58 & 1 & 12 & 1,03 \\
\hline Geral & 12.536 & 1,59 & 87 & 36 & 4.900 & 184 & 1,82 & 1 & 12 & 1,67 \\
\hline Joalherias & 9.818 & 1,25 & 150 & 68 & 2.530 & 205 & 3,10 & 2 & 12 & 2,53 \\
\hline Livraria & 10.118 & 1,28 & 82 & 41 & 2.658 & 128 & 2,13 & 1 & 15 & 1,80 \\
\hline Lojas em geral & 60.993 & 7,74 & 98 & 47 & 6.030 & 176 & 2,61 & 2 & 15 & 2,39 \\
\hline Móveis & 7.473 & 0,95 & 313 & 184 & 3.890 & 379 & 4,87 & 4 & 12 & 3,61 \\
\hline Perfumaria & 14.266 & 1,81 & 74 & 44 & 3.106 & 112 & 1,99 & 2 & 10 & 1,29 \\
\hline Postos de gasolina & 76.863 & 9,75 & 37 & 30 & 1.041 & 33 & 1,05 & 1 & 10 & 0,27 \\
\hline Serviços & 20.481 & 2,60 & 89 & 40 & 3.000 & 151 & 1,65 & 1 & 12 & 1,46 \\
\hline Supermercado & 267.307 & 33,92 & 83 & 35 & 5.555 & 175 & 2,07 & 1 & 18 & 2,57 \\
\hline $\begin{array}{l}\text { Telecomunicações } \\
\text { / Eletrônicos }\end{array}$ & 25.809 & 3,28 & 153 & 45 & 3.480 & 273 & 3,04 & 1 & 12 & 3,21 \\
\hline Vestuário & 87.230 & 11,07 & 88 & 60 & 3.303 & 103 & 2,62 & 2 & 12 & 1,68 \\
\hline Veículos & 18.721 & 2,38 & 143 & 75 & 5.000 & 213 & 2,35 & 2 & 12 & 1,67 \\
\hline Viagens & 5.251 & 0,67 & 157 & 48 & 3.610 & 260 & 1,98 & 1 & 12 & 1,77 \\
\hline Total & 787.986 & 100,0 & 81 & 36 & 6.845 & 161 & 2,00 & 1 & 18 & 2,10 \\
\hline
\end{tabular}

Tabela 13: Total de transações, frequência relativa percentual, valores médios, medianos, máximos e planos de pagamentos médios, medianos e máximos por família de produtos.

A Figura 9 apresenta as recorrências de consumo no ano e no mês para cada uma das famílias de produtos estudadas. A recorrência é a quantidade de vezes que um cliente compra produtos da mesma família em um determinado período de observação.

As famílias de produtos apresentam recorrências diferentes entre as observações mensais ou anuais.

Observou-se na base de transações que cada cliente que realizou ao menos uma compra no ano em supermercado - família de produto com o maior volume de transações - comprou, em média, 12,3 vezes em supermercados no ano, utilizando o cartão estudado como meio de pagamento. Analisando-se a recorrência de compras no mesmo mês, os clientes com compras em supermercados, fizeram em média 2,7 compras desta família de produtos. 


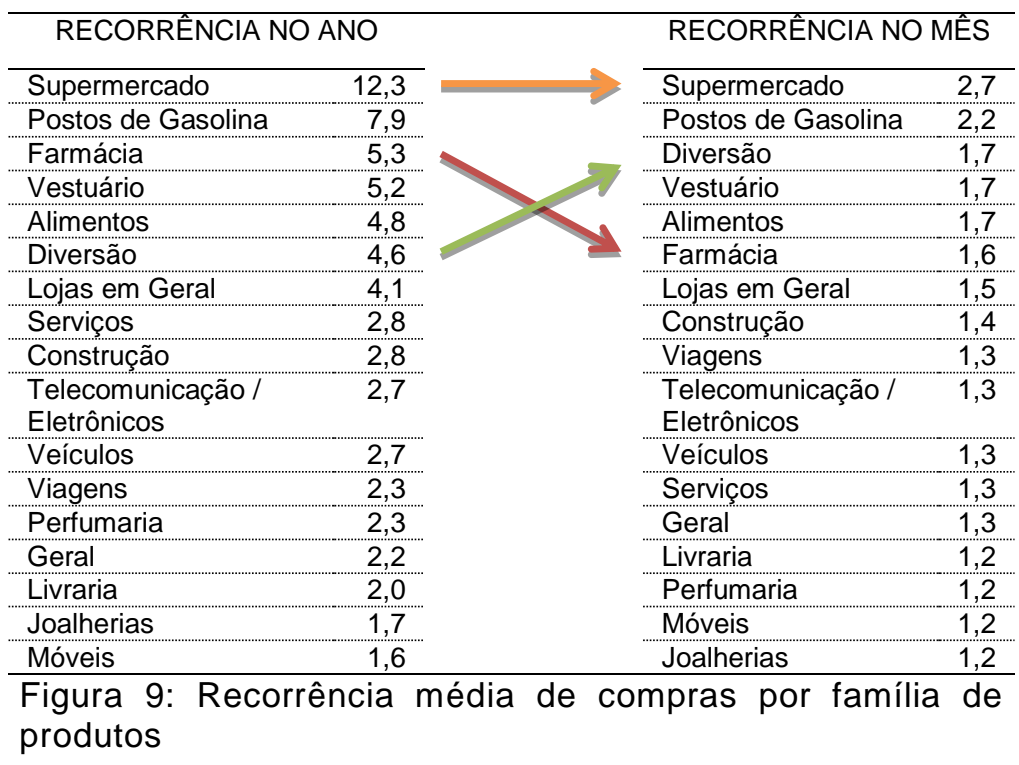

Nota-se que existem inversões de posição em alguns casos, o que pode sugerir a seguinte leitura: em média, quem compra em farmácias tem uma recorrência anual $(5,3)$ superior à recorrência de quem consome em diversão $(4,6)$. No entanto, a recorrência no mês em diversão é maior $(1,7)$ do que a recorrência mensal de compras em farmácia $(1,6)$.

A conclusão a que se pode atribuir para este fato é que, embora com frequência inferior em relação à farmácia, no intervalo de um ano, o cliente consuma diversão; no mês em que se consome diversão, consome mais vezes do que nos meses em que se consome farmácia.

Outras inversões também podem ser percebidas na figura, de modo a oferecerem dados importantes para a segmentação dos clientes.

\subsection{Aplicação da análise de associação à base de modelagem}

$\mathrm{Na}$ aplicação da AA é necessário realizar a análise gráfica e das regras de associação. Como resultado da aplicação tem-se um documento com as principais regras de relacionamento, demonstrado no Apêndice 2.

A seguir, apresentam-se oito blocos de análises realizadas separadamente. Cada um dos blocos demonstra a análise e um conjunto de 20 regras de 
associação, sendo as 10 de maior lift e as 10 de maior support, cuja tabela com as principais regras, é apresentada no Apêndice 2.

Primeiramente têm-se quatro análises cuja associação entre as transações se dá a cada mês.

Ressalta-se que foi considerada a relação entre as compras de produtos incluídas em uma mesma fatura de cartão de crédito de cada cliente e a base de faturas analisada possui 26.767 clientes, 171.667 faturas e 787.986 transações ou compras de produtos.

Em segundo lugar outras quatro análises tomando por base todas as compras dos clientes no ano, ou seja, 26.767 clientes com 787.986 transações.

\subsubsection{Análise de associação das famílias de produtos nas compras mensais}

As famílias de produtos, descritas na Tabela 11, são 17 agrupamentos de ramos de atividades do comércio de produtos e serviços que fazem parte da cadeia de valor do cartão de crédito.

A Figura 10 apresenta o resultado da aplicação da $A A$ às transações realizadas pelos clientes durante cada um dos meses do período.

O tamanho e a cor das circunferências - denominadas nós - representam a quantidade de transações associadas a cada família de produto. Percebe-se a variação de tamanho associada à variação de cor. O uso da cor vermelha está associado a valores altos e o uso do azul está associado a valores baixos. Sendo assim, uma circunferência vermelha representando uma família de produtos indica que a frequência de compras de produto desta família é maior do que a frequência de consumo dos produtos de uma família representada por uma circunferência em azul. 
Cada seta representa uma regra de associação. A largura e a cor da seta indicam o valor confidence, que é a probabilidade de ocorrer uma transação consequente, dado que houve uma transação antecedente. Uma seta larga e vermelha entre duas famílias de produtos significa que existe alta probabilidade do consumo de um produto da família indicada pela ponta da seta, dado o consumo antecedente do produto da família representada pela circunferência na origem da seta; ao passo que uma associação descrita por uma seta estreita e azul demonstra uma probabilidade menor.

Com o auxílio da Figura 10 pode-se verificar que famílias de produtos estratégicas concentram boa parte das transações associadas. A família de produtos posto de gasolina está associada fortemente como consequente da combinação veículos e diversão. Juntamente às categorias destacadas, alimentos, serviços e perfumaria, demonstram serem famílias de produtos estratégicas e possuem várias associações com outras famílias. 

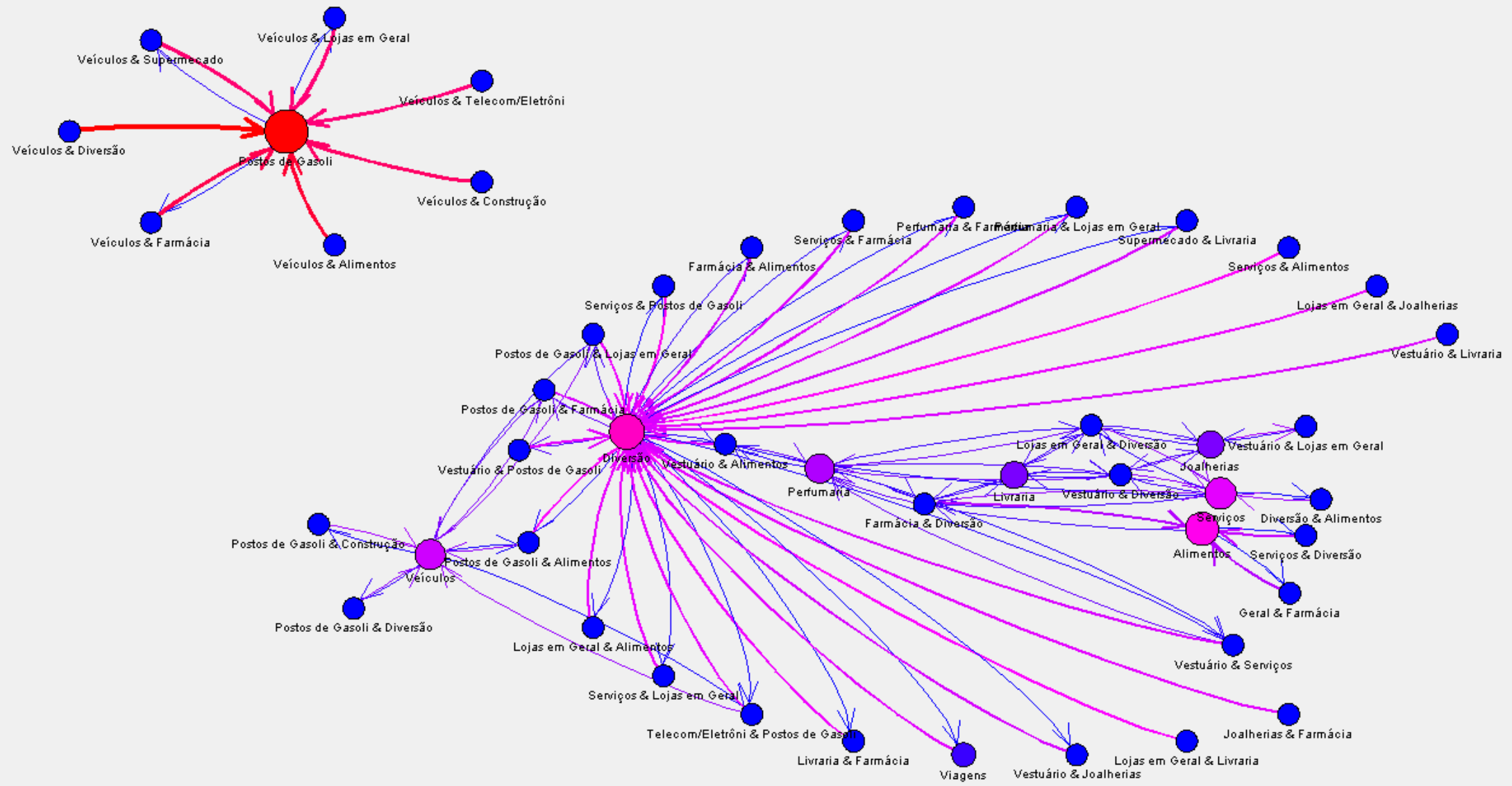

Figura 10: Grafo de associações de famílias de produtos por mês 
Para uma avaliação da aplicação da técnica da AA na base transacional de clientes, apresenta-se uma sequência de gráficos que podem auxiliar na percepção e nos destaques das regras que podem ser utilizadas para a segmentação dos clientes.

No Gráfico 2, é apresentado o support e o confidence das cem primeiras regras de associação de maior lift. Destaca-se neste gráfico a regra de número 74 , que tem o seguinte enunciado: Postos de Gasolina \& Farmácia ==> Diversão. Esta regra se destaca pelo alto valor atribuído ao support, frente às demais e significa que ao observar um cliente com transações em posto de gasolina e farmácia, espera-se encontrar 2,43 vezes mais clientes que compraram produtos da família diversão do que selecionando um cliente entre todos os observados. O valor do lift mencionado $(2,43)$, bem como as demais informações das principais regras utilizadas na modelagem constam na Tabela 48 do Apêndice 2.

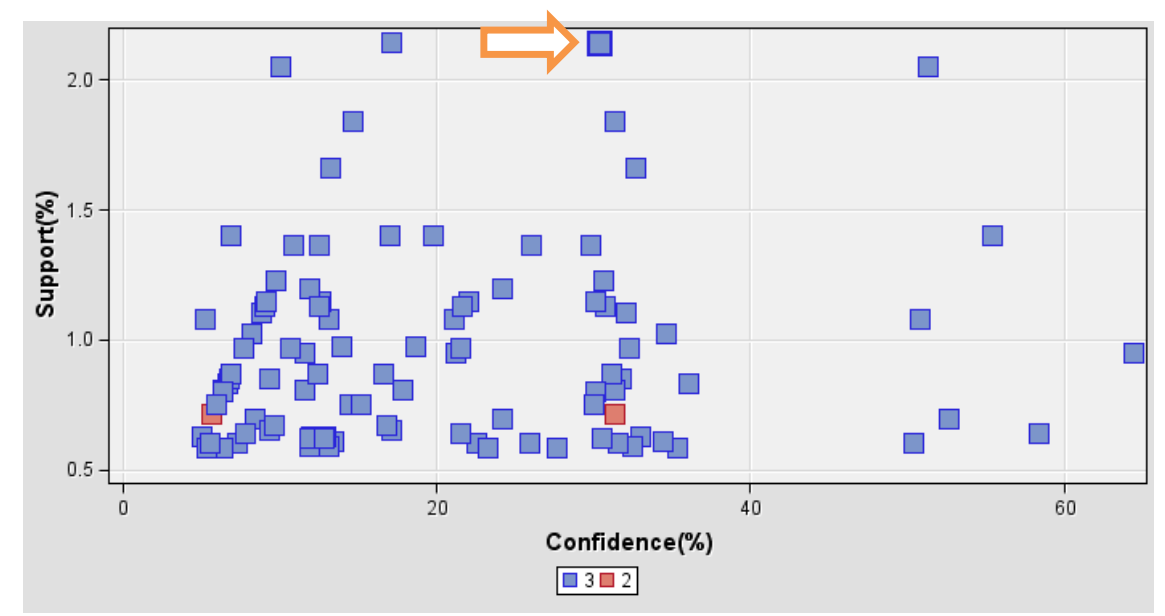

Gráfico 2: Support versus confidence para as cem primeiras regras de associação das famílias de produtos por mês.

O Gráfico 3 apresenta o confidence, expected confidence, lift e support. Para exemplificar a análise aplicada a todas as régras de associação, segue em destaque a regra (74).

É importante verificar que nas primeiras cem regras há uma variação pequena no lift, que tem como valor máximo 3,18 e mínimo 2,34. Contudo o conjunto total das regras de associação encontradas, com 1.210 regras tem o menor lift de 0,85 , para 
a regra Móveis ==> Supermercado. Esta regra indica que existe uma repulsão a compras em Supermercados para clientes que tiveram compras de Móveis.

O maior support encontrado foi de 15,27 para a regra Supermercado ==> Farmácia, indicando que $15,27 \%$ dos consumidores apresentaram esta combinação de transações. A regra de menor support, Serviços ==> Perfumaria \& Farmácia foi de 0,57 e, embora pouco presente nas transações observadas, apresenta lift de 2,28 .

As variações do confidence e do expected confidence, são efeitos menos importantes, já que são cálculos intermediários para a obtenção do lift.

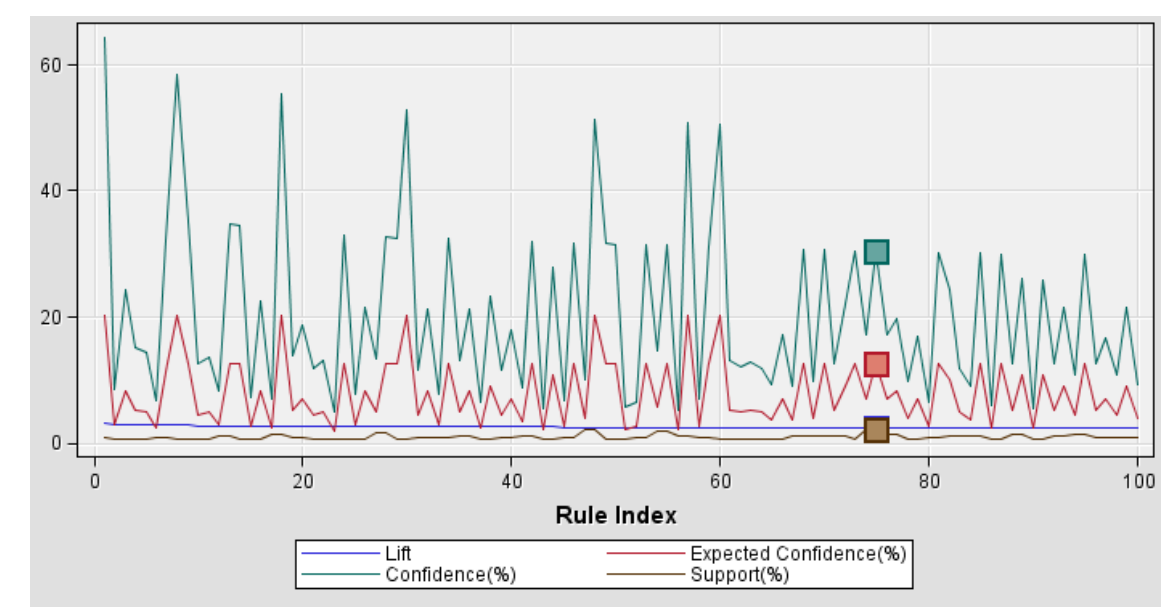

Gráfico 3: Lift, confidence, expected confidence e support de família de produtos por mês

Finalizando a seguência de gráficos que auxiliam na avaliação da aplicação da técnica de $A A$, apresenta-se uma matriz de regras de associação (Figura 11) cujos eixos representam os antecedentes e os consequentes, enquanto as cores, na escala de azul até vermelho, representam confidence, ainda com o destaque para a regra de número 74 (Posto de Gasolina \& Farmácia ==> Diversão). 


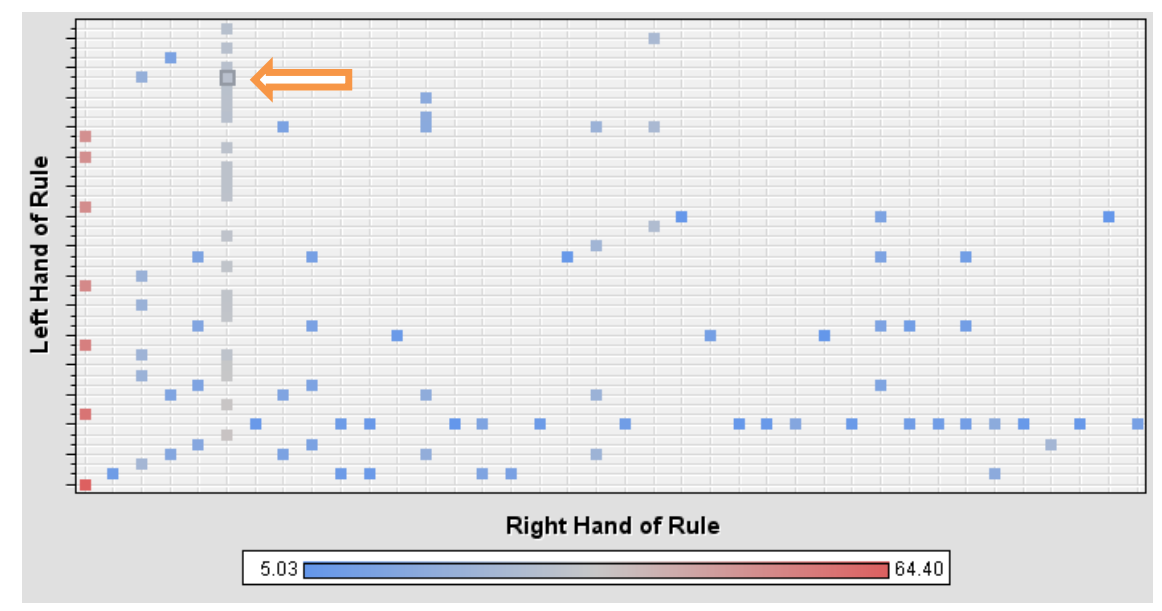

Figura 11: Matriz de regras de associação de família de produtos por mês

Analisando a matriz, percebe-se que muitas outras famílias de produtos ou combinações delas são associadas à diversão por meio da quantidade de pontos na mesma coluna. Além disso, nota-se que o indicador de força da associação (confidence) está na posição central da escala (com a cor cinza). A primeira coluna da matriz, do consequente Posto de Gasolina, tem as ligações menos numerosas, porém mais fortes, conforme demonstrado pela cor dos seus pontos.

Observando-se estas representações conclui-se que, ao testar a associação das famílias de produtos consumidos em um mesmo mês, distingue-se algumas regras que se são importantes para a segmentação dos clientes.

Nas Tabelas 14 e 15, apresentam as 10 regras de maior lift e de maior support desta análise. Estas regras de associação são utilizadas no modelo de segmentação de clientes, juntamente com os outros sete conjuntos de regras oriundos das análises. 


\begin{tabular}{|c|c|c|c|c|c|c|}
\hline $\begin{array}{c}\text { Expected } \\
\text { Confidence } \\
(\%)\end{array}$ & $\begin{array}{c}\text { Confidence } \\
(\%)\end{array}$ & $\begin{array}{c}\text { Support } \\
(\%)\end{array}$ & Lift & Transações & Regra & ID da Regra \\
\hline 20,25 & 64,40 & 0,95 & 3,18 & 1628,0 & $\begin{array}{c}\text { Veículos \& Diversão } \\
==>\text { Postos de } \\
\text { Gasolina }\end{array}$ & 1 \\
\hline 2,87 & 8,49 & 0,70 & 2,96 & 1194,0 & $\begin{array}{c}\text { Veículos }==>\text { Postos } \\
\text { de Gasolina \& } \\
\text { Construcão }\end{array}$ & 2 \\
\hline 8,19 & 24,25 & 0,70 & 2,96 & 1194,0 & $\begin{array}{c}\text { Postos de Gasolina } \\
\text { \& Construção ==> } \\
\text { Veículos }\end{array}$ & 3 \\
\hline 4,94 & 14,45 & 0,75 & 2,92 & 1287,0 & $\begin{array}{c}\text { Vestuário \& } \\
\text { Diversão ==> } \\
\text { Joalherias }\end{array}$ & 5 \\
\hline 5,19 & 15,17 & 0,75 & 2,92 & 1287,0 & $\begin{array}{c}\text { Joalherias }==> \\
\text { Vestuário \& } \\
\text { Diversão }\end{array}$ & 4 \\
\hline 12,50 & 36,10 & 0,83 & 2,89 & 1430,0 & $\begin{array}{c}\text { Serviços \& Postos } \\
\text { de Gasolina ==> } \\
\text { Diversão }\end{array}$ & 7 \\
\hline 2,31 & 6,66 & 0,83 & 2,89 & 1430,0 & $\begin{array}{l}\text { Diversão ==> } \\
\text { Serviços \& Postos } \\
\text { de Gasolina }\end{array}$ & 6 \\
\hline 20,25 & 58,43 & 0,64 & 2,89 & 1095,0 & $\begin{array}{c}\text { Veículos \& } \\
\text { Alimentos }==> \\
\text { Postos de Gasolina }\end{array}$ & 8 \\
\hline 12,50 & 35,33 & 0,58 & 2,83 & 998,00 & $\begin{array}{c}\text { Serviços \& } \\
\text { Alimentos ==> } \\
\text { Diversão }\end{array}$ & 9 \\
\hline 4,84 & 13,50 & 0,61 & 2,79 & 1041,0 & $\begin{array}{c}\text { Lojas em Geral \& } \\
\text { Diversão ==> } \\
\text { Livraria }\end{array}$ & 11 \\
\hline
\end{tabular}

Tabela 14: Regras de maior lift - família de produtos por mês

Observa-se na Tabela 15 que as regras com altos valores atribuídos ao support, demonstram o lift baixo e próximo a 1. Conclui-se então, que muitas regras encontradas na análise não devem ser consideradas no modelo de segmentação.

\begin{tabular}{|c|c|c|c|c|c|c|}
\hline $\begin{array}{c}\text { Expected } \\
\text { Confidence } \\
(\%)\end{array}$ & $\begin{array}{c}\text { Confidence } \\
(\%)\end{array}$ & $\begin{array}{c}\text { Support } \\
(\%)\end{array}$ & Lift & Transações & Regra & ID da Regra \\
\hline 27,23 & 26,89 & 15,27 & 0,99 & 26216 & $\begin{array}{c}\text { Supermercado }==> \\
\text { Farmácia }\end{array}$ & 1185 \\
\hline 56,78 & 56,08 & 15,27 & 0,99 & 26216 & $\begin{array}{l}\text { Farmácia ==> } \\
\text { Supermercado }\end{array}$ & 1186 \\
\hline 56,78 & 49,98 & 14,77 & 0,88 & 25356 & $\begin{array}{l}\text { Vestuário ==> } \\
\text { Supermercado }\end{array}$ & 1206 \\
\hline 29,55 & 26,01 & 14,77 & 0,88 & 25356 & $\begin{array}{c}\text { Supermercado } \\
\text { Vestuário }\end{array}==>$ & 1207 \\
\hline 23,13 & 21,24 & 12,06 & 0,92 & 20701 & $\begin{array}{c}\text { Supermercado }==> \\
\text { Lojas em Geral }\end{array}$ & 1201 \\
\hline 56,78 & 52,13 & 12,06 & 0,92 & 20701 & $\begin{array}{l}\text { Lojas em Geral ==> } \\
\text { Supermercado }\end{array}$ & 1202 \\
\hline 20,25 & 19,83 & 11,26 & 0,98 & 19332 & $\begin{array}{l}\text { Supermercado ==> } \\
\text { Postos de Gasolina }\end{array}$ & 1189 \\
\hline 56,78 & 55,61 & 11,26 & 0,98 & 19332 & $\begin{array}{l}\text { Postos de Gasolina } \\
==>\text { Supermercado }\end{array}$ & 1190 \\
\hline 27,23 & 34,39 & 10,16 & 1,26 & 17447 & $\begin{array}{c}\text { Vestuário ==> } \\
\text { Farmácia }\end{array}$ & 1034 \\
\hline 29,55 & 37,32 & 10,16 & 1,26 & 17447 & $\begin{array}{c}\text { Farmácia }==> \\
\text { Vestuário }\end{array}$ & 1035 \\
\hline
\end{tabular}

Tabela 15: Regras de maior support - família de produtos por mês 
5.2.2 Análise de associação dos produtos nas compras mensais

Os produtos, conforme descritos na Seção 4.4 .1 foram definidos a partir da família de produtos, do plano de pagamento e da faixa de valor do produto (conforme a Tabela 12).

A associação entre os produtos nas compras mensais demonstrou muitos casos em que os produtos se associam a outros produtos da mesma família. Com isso, percebe-se que há elevada recorrência do uso do cartão em estabelecimentos que comercializam os mesmos tipos de bens. Entretanto, algumas associações entre produtos de famílias diferentes também ocorrem, como na regra de número cinco, descrita pelo enunciado abaixo:

Alimentos_1_F01:0|-12 => Farmacia_1_F01:0|-11 \& Alimentos_1_F02:12|-21

As cem primeiras regras, do ponto de vista do lift, estão ilustradas nas Figuras 12 e 13. O grafo foi partido em duas figuras para possibilitar melhor visualização. Estas regras indicam produtos estratégicos: os de maior dimensão e quantidade de associações a outros produtos. 


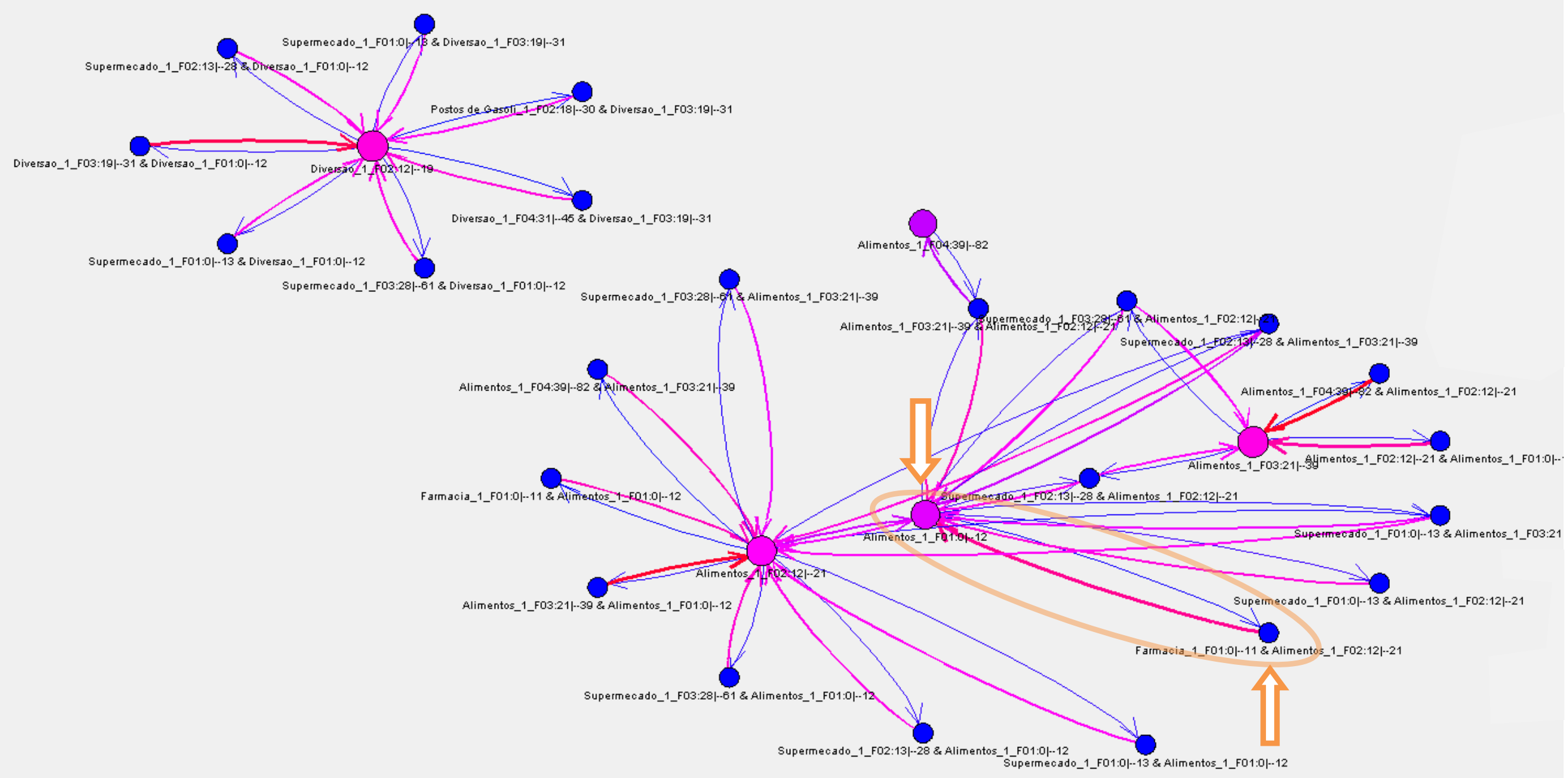

Figura 12: Grafo de associações de produtos por mês (parte 1) 


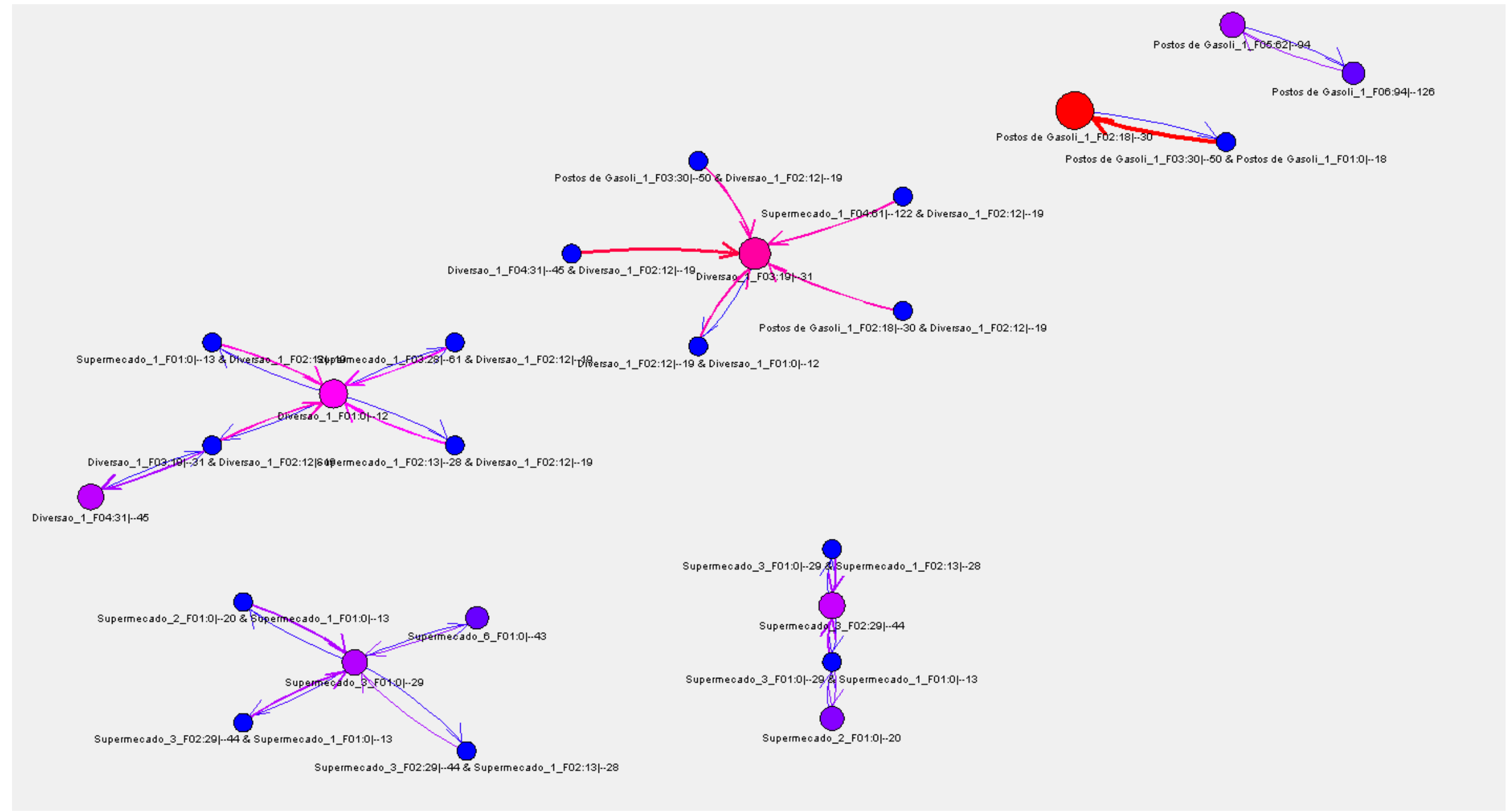

Figura 13: Grafo de associações de produtos por mês (parte 2) 
Nas Figuras 12 e 13 observam-se em destaque produtos das famílias diversão, alimentos, supermercado, e posto de gasolina. Na Figura 12, observa-se em destaque a regra de associação entre os produtos "Farmácia_1_F01:0|-11" em conjunto com "Alimentos_1_F02:12|-21", que indica o produto "Alimentos_1_F01:0|12" (regra número 6). A regra de associação no sentido contrário (regra número 5) é demonstrada por uma seta azul e estreita, o que significa que o confidence desta associação é menor do que a primeira descrita.

A regra cinco está marcada em destaque nos Gráficos 4, 5 e na Figura 14. Embora apresente baixo valor de support ou confidence, em relação às demais regras, possui um valor elevado atribuído ao lift.

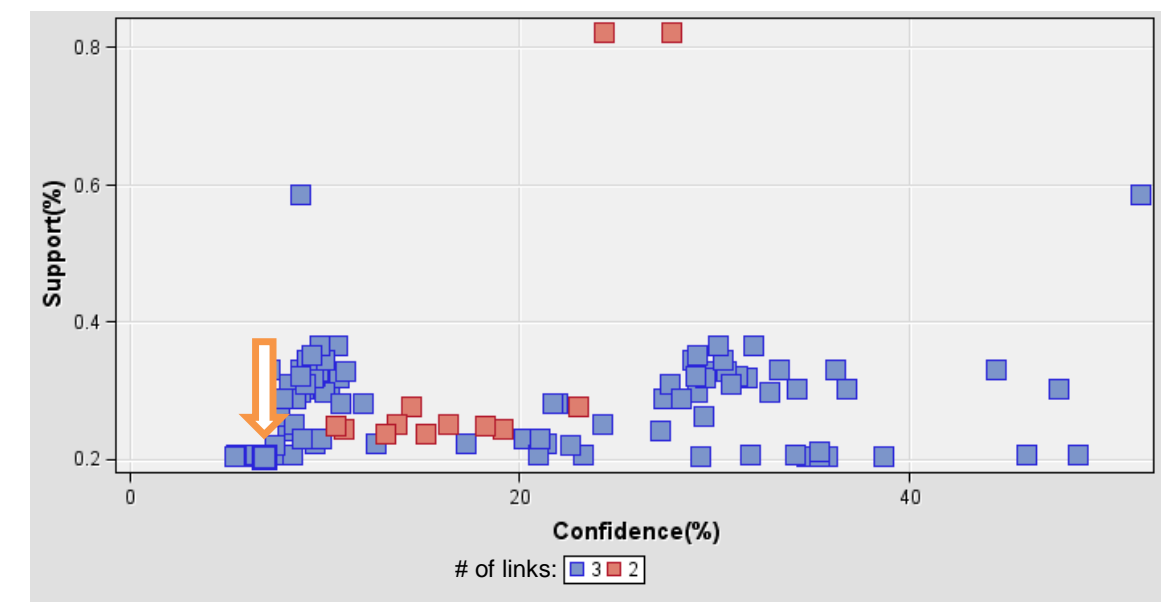

Gráfico 4: Support versus confidence para as cem primeiras regras de associação de produtos por mês

O Gráfico 5 apresenta o confidence, expected confidence, lift e support. Conclui-se que o lift das cem primeiras regras compreende valores elevados, o que indica que muitas regras, provenientes desta análise, serão importantes no modelo de segmentação. 


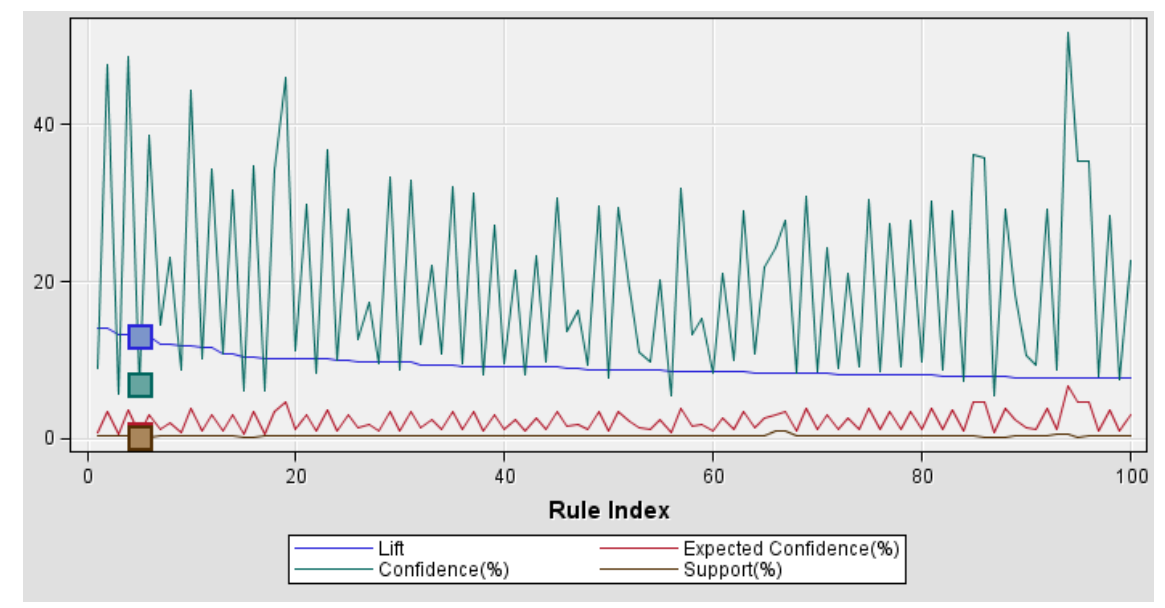

Gráfico 5: Lift, confidence, expected confidence e support de produtos por mês

A matriz de regras, apresentada na Figura 14, apresenta que apenas a associação descrita pela regra cinco tem como consumo consequente a combinação dos produtos "Farmacia_1_F01:0|-11" e "Alimentos_1_F02:12|-21", entre as cem primeiras regras. No entanto, o produto antecedente "Alimentos_1_F01:0|-12" é associado a outros sete produtos ou combinações de produtos consequentes.

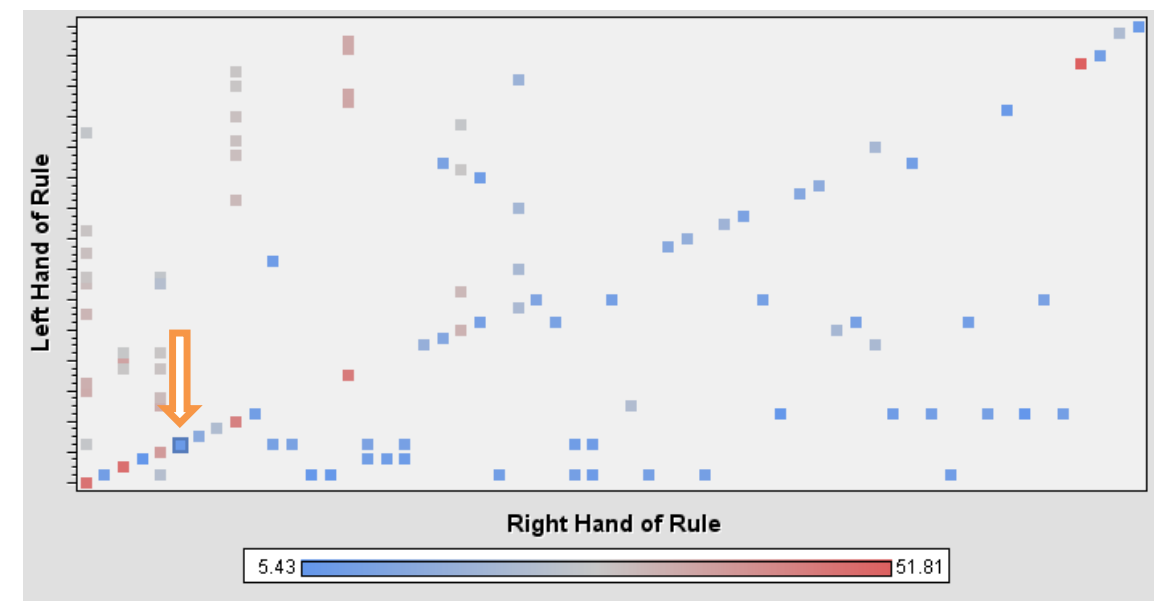

Figura 14: Matriz de regras de associação de produtos por mês

A Tabela 16 apresenta as dez regras de maior lift da análise. Observa-se que os valores do support são da ordem de $0,3 \%$ da base transacional. 


\begin{tabular}{|c|c|c|c|c|c|c|}
\hline $\begin{array}{c}\text { Expected } \\
\text { Confidence } \\
(\%)\end{array}$ & $\begin{array}{c}\text { Confidence } \\
(\%)\end{array}$ & $\begin{array}{c}\text { Support } \\
(\%)\end{array}$ & Lift & Transações & Regra & $\begin{array}{l}\text { ID da } \\
\text { Regra }\end{array}$ \\
\hline 3,38 & 47,66 & 0,30 & 14,09 & 520,00 & $\begin{array}{c}\text { Alimentos_1_F03:21|--39 \& } \\
\text { Alimentos_1_F01:0|--12 ==> } \\
\text { Alimentos_1 F02:12|--21 }\end{array}$ & 2 \\
\hline 0,64 & 8,96 & 0,30 & 14,09 & 520,00 & $\begin{array}{c}\text { Alimentos_1_F02:12|--21 } \\
==>\text { Alimentos_1_F03:21|-- } \\
\text { 39 \& Alimentos_1_F01:0|-- } \\
\text { 12 }\end{array}$ & 1 \\
\hline 3,66 & 48,63 & 0,21 & 13,27 & 354,00 & $\begin{array}{c}\text { Alimentos_1_F04:39|--82 \& } \\
\text { Alimentos_1_F02:12|--21 } \\
==>\text { Alimentos_1_F03:21|-- } \\
39\end{array}$ & 4 \\
\hline 0,42 & 5,63 & 0,21 & 13,27 & 354,00 & $\begin{array}{c}\text { Alimentos_1_F03:21|--39 } \\
==>\text { Alimentos_1_F04:39|-- } \\
82 \text { \& Alimentos_1_F02:12|-- } \\
\text { 21 }\end{array}$ & 3 \\
\hline 2,96 & 38,67 & 0,20 & 13,06 & 350,00 & $\begin{aligned} & \text { Farmacia_1_F01:0|--11 \& } \\
& \text { Alimentos_1_F02:12|--21 } \\
&==>\text { Alimentos_1_F01:0|--12 }\end{aligned}$ & 6 \\
\hline 0,53 & 6,88 & 0,20 & 13,06 & 350,00 & $\begin{array}{l}\text { Alimentos_1_F01:0|--12 ==> } \\
\text { Farmacia_1_F01:0|--11 \& } \\
\text { Alimentos_1_F02:12|--21 }\end{array}$ & 5 \\
\hline 1,20 & 14,49 & 0,28 & 12,03 & 475,00 & $\begin{array}{c}\text { Telecom/Eletr_1_F02:12|-- } \\
20==> \\
\text { Telecom/Eletr_1_F01:0|--12 }\end{array}$ & 7 \\
\hline 1,91 & 22,98 & 0,28 & 12,03 & 475,00 & $\begin{array}{c}\text { Telecom/Eletr_1_F01:0|--12 } \\
==> \\
\text { Telecom/Eletr_1_F02:12|-- } \\
20\end{array}$ & 8 \\
\hline 3,76 & 44,42 & 0,33 & 11,82 & 565,00 & $\begin{array}{c}\text { Diversao_1_F03:19|--31 \& } \\
\text { Diversao_1_F01:0|--12==> } \\
\text { Diversao_1_F02:12|--19 }\end{array}$ & 10 \\
\hline 0,74 & 8,76 & 0,33 & 11,82 & 565,00 & $\begin{array}{l}\text { Diversao_1_F02:12|-19 } \\
==>\text { Diversao_1_F03:19|-- } \\
\text { 31 \& Diversao_1_F01:0|--12 }\end{array}$ & 9 \\
\hline
\end{tabular}

Tabela 16: Regras de maior lift - produtos por mês

$\mathrm{Na}$ Tabela 17 observa-se regras com support elevado e valores de lift superiores ou próximos a 2 . A utilização destas regras resulta em ganhos de eficiência aplicada a maior quantidade de clientes do que as enunciadas na Tabela 16. 


\begin{tabular}{|c|c|c|c|c|c|c|}
\hline $\begin{array}{c}\text { Expected } \\
\text { Confidence } \\
(\%)\end{array}$ & $\begin{array}{c}\text { Confidence } \\
(\%)\end{array}$ & $\begin{array}{c}\text { Support } \\
(\%)\end{array}$ & Lift & Transações & Regra & $\begin{array}{l}\text { ID da } \\
\text { Regra }\end{array}$ \\
\hline 19,11 & 38,38 & 7,74 & 2,01 & 13282 & $\begin{array}{c}\text { Supermercado_1_F03:28|--61 } \\
==>\text { Supermercado_1_F02:13|- } \\
-28\end{array}$ & 1471 \\
\hline 20,16 & 40,49 & 7,74 & 2,01 & 13282 & $\begin{array}{c}\text { Supermercado_1_F02:13|--28 } \\
==>\text { Supermercado_1_F03:28|- } \\
-61\end{array}$ & 1472 \\
\hline 15,78 & 39,21 & 7,49 & 2,48 & 12862 & $\begin{array}{l}\text { Supermercado_1_F02:13|--28 } \\
==>\text { Supermercado_1_F01:0|-- } \\
13\end{array}$ & 992 \\
\hline 19,11 & 47,47 & 7,49 & 2,48 & 12862 & $\begin{array}{c}\text { Supermercado_1_F01:0|--13 } \\
==>\text { Supermercado_1_F02:13|- } \\
-28\end{array}$ & 993 \\
\hline 15,78 & 30,56 & 6,16 & 1,94 & 10577 & $\begin{array}{l}\text { Supermercado_1_F03:28|--61 } \\
==>\text { Supermercado_1_F01:0|-- } \\
13\end{array}$ & 1556 \\
\hline 20,16 & 39,04 & 6,16 & 1,94 & 10577 & $\begin{array}{c}\text { Supermercado_1_F01:0|--13 } \\
==>\text { Supermercado_1_F03:28|- } \\
-61\end{array}$ & 1557 \\
\hline 20,16 & 37,41 & 4,74 & 1,86 & 8133,0 & $\begin{array}{l}\text { Supermercado_1_F04:61|--122 } \\
==>\text { Supermercado_1_F03:28|- } \\
-61\end{array}$ & 1642 \\
\hline 12,66 & 23,50 & 4,74 & 1,86 & 8133,0 & $\begin{array}{c}\text { Supermercado_1_F03:28|--61 } \\
==>\text { Supermercado_1_F04:61|- } \\
-122\end{array}$ & 1643 \\
\hline 19,11 & 30,66 & 3,88 & 1,60 & 6666,0 & $\begin{array}{c}\text { Supermercado_1_F04:61|--122 } \\
==>\text { Supermercado_1_F02:13|- } \\
-28\end{array}$ & 1924 \\
\hline 12,66 & 20,32 & 3,88 & 1,60 & 6666,0 & $\begin{array}{c}\text { Supermercado_1_F02:13|--28 } \\
==>\text { Supermercado_1_F04:61|- } \\
-122\end{array}$ & 1925 \\
\hline
\end{tabular}

Tabela 17: Regras de maior support-produtos por mês

5.2.3 Análise de associação dos planos de pagamentos nas compras mensais

Ao analisar a associação dos planos de pagamento adotados pelos clientes, observa-se as duas primeiras regras, segundo o lift $(2,44)$ : "10 \& $3==>$ "; "6 ==> 10 \& 3". Embora as duas regras tenham o mesmo valor de lift, seus valores de confidence são bastante distantes.

A Figura 15 apresenta graficamente a relação das cem primeiras regras, segundo o lift para os planos de pagamentos adotados pelos clientes nas transações. As transações com maior volume e quantidade de associações são realizadas em duas ou três parcelas. 


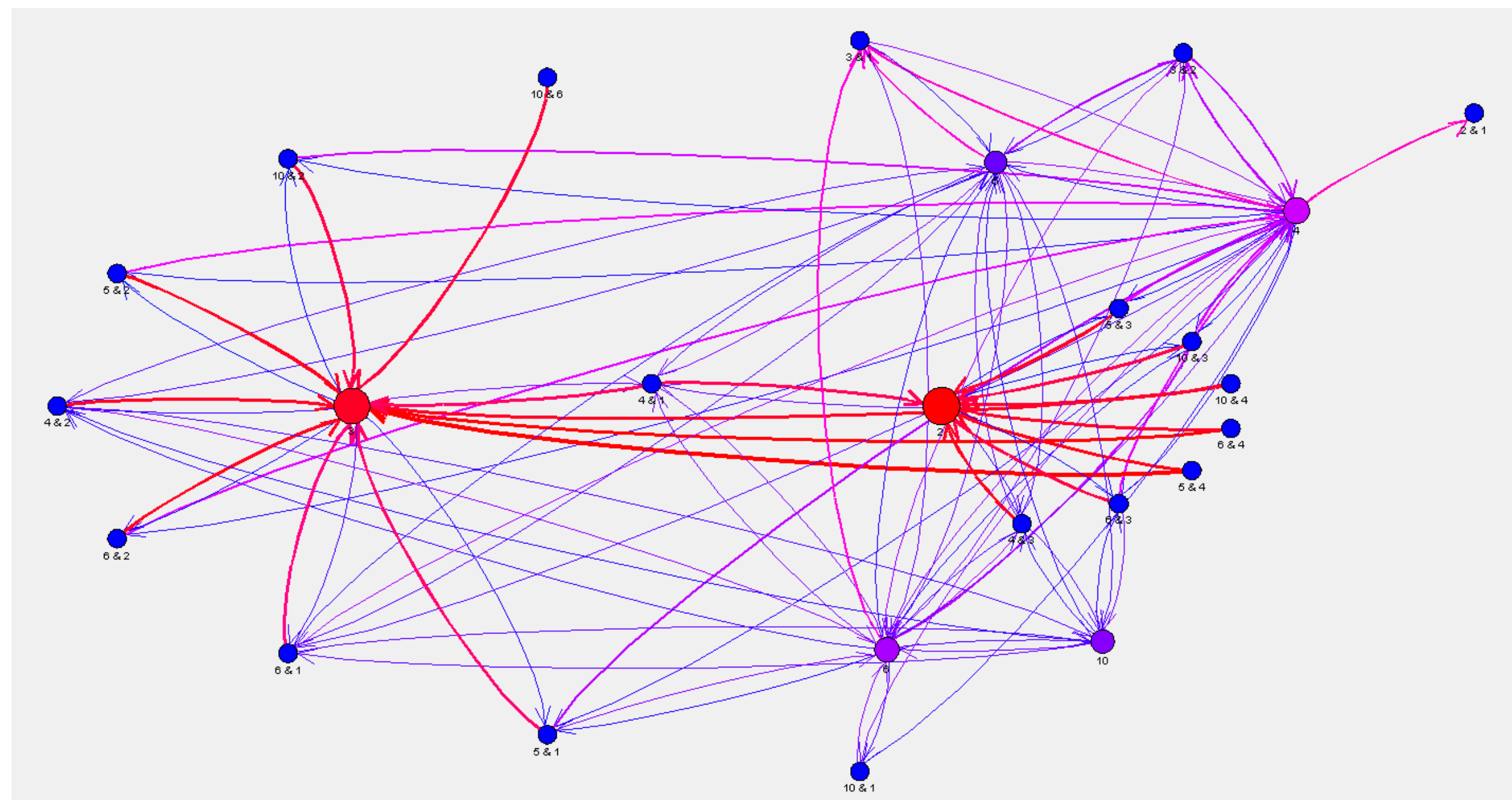

Figura 15: Grafo de associações de planos de pagamento por mês 
O Gráfico 6 apresenta o diagrama de support e confidence dispersão das cem primeiras regras de associação entre os planos de pagamento das transações realizadas a cada mês. A maior concentração das regras está no intervalo entre $5 \%$ e $30 \%$ de confidence, e abaixo dos $2 \%$ de support.

Nos Gráficos 6 e 7 e Figura 16, destaca-se a regra de maior lift $(2,44)$, representada por $10 \& 3==>6$.

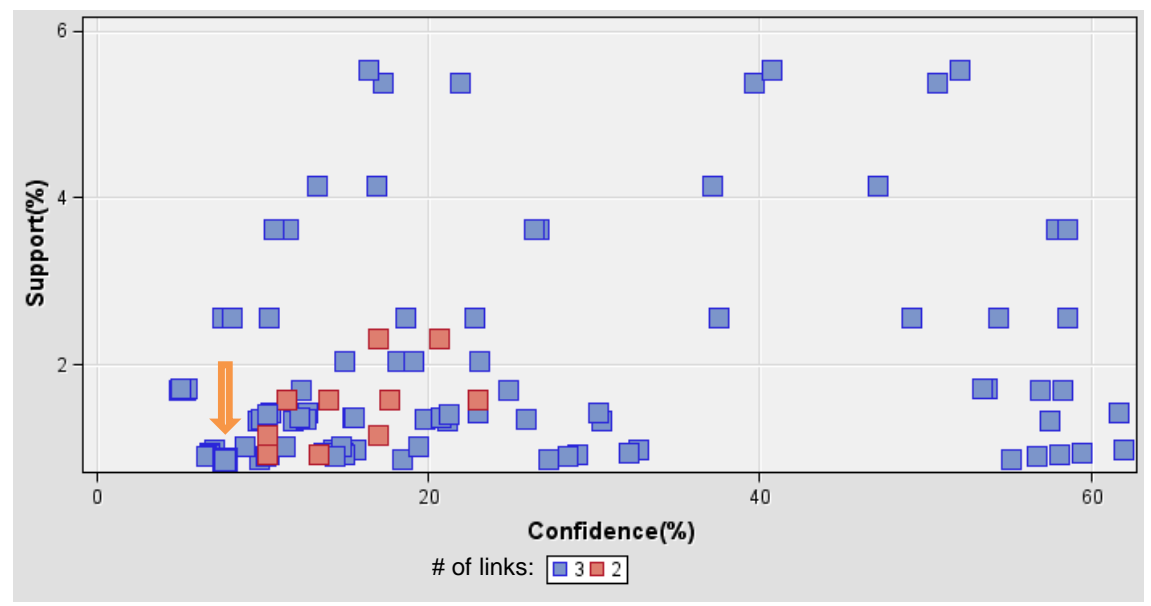

Gráfico 6: Support versus confidence para as cem primeiras regras de associação de planos de pagamentos por mês

O Gráfico 7 apresenta o confidence, expected confidence, lift e support. $\mathrm{Na}$ avaliação dos indicadores demonstrados, observa-se o lift praticamente constante com valores baixos, indica que o plano de pagamento agregará pouco no modelo de segmentação de clientes, uma vez que os valores encontrados de lift não são elevados. 


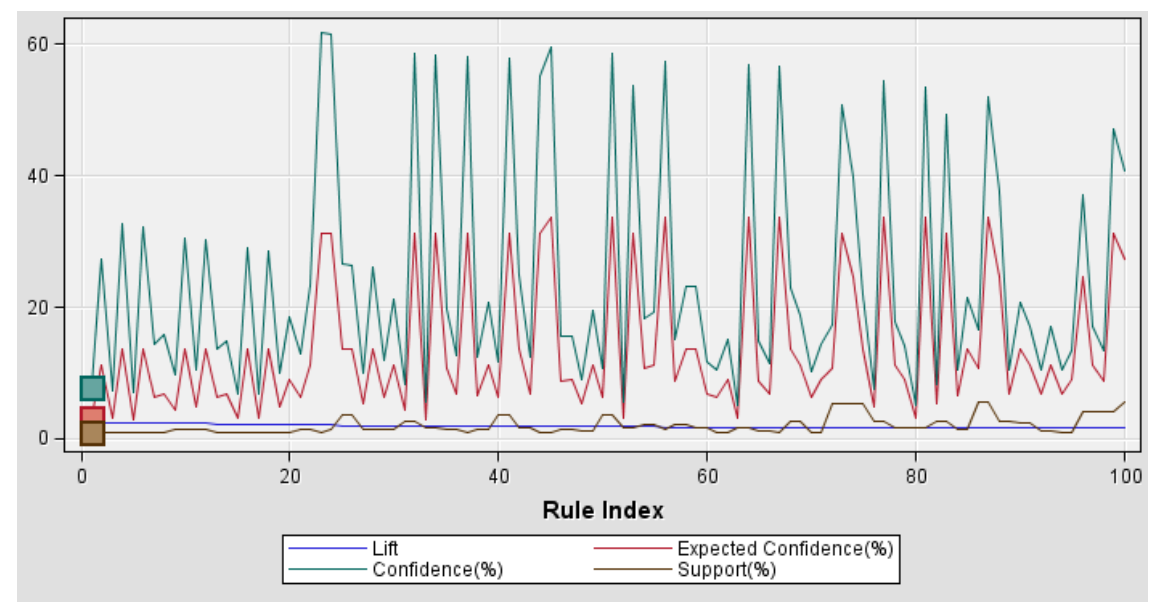

Gráfico 7: Lift, confidence, expected confidence e support de planos de pagamentos por mês

A Figura 16 apresenta a distribuição das regras encontradas na análise entre antecedentes e consequentes e, a partir dela percebe-se que poucas regras têm valores elevados de confidence, representando a força entre as associações.

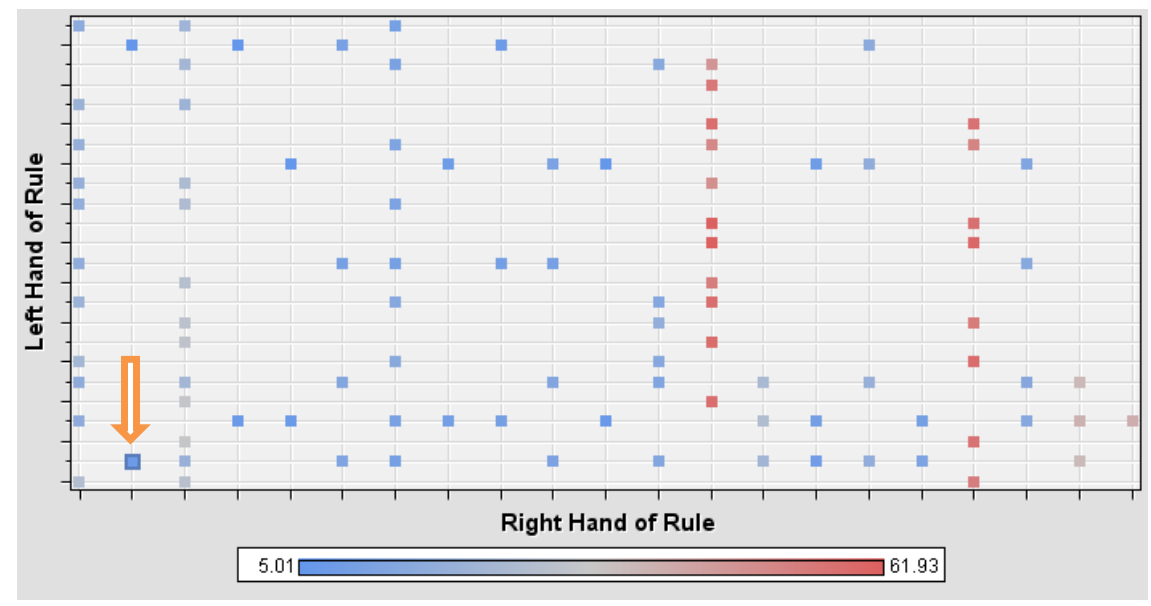

Figura 16: Matriz de regras de associação de planos de pagamentos por mês

A Tabela 18 explicita a diferença entre a primeira e a segunda regras enunciadas, sobretudo na ordem de grandeza do indicador confidence. A interpretação deste indicador para a regra de número 1 é de que $7,75 \%$ dos clientes que possuem transações realizadas em 6 parcelas possuem também transações realizadas em 10 e em 3 parcelas, enquanto que 27,24\% dos clientes que possuem transações realizadas em 10 e em 3 parcelas possuem também transações realizadas em 6 parcelas, ou seja, as relações entre os parcelamentos de transações ocorrem com diferentes intensidades. No entanto, nas duas regras, é 
possível verificar o lift de 2,44, que significa que dado o fator antecedente, as chances de se encontrar o fator consequente aumentam 2,44 vezes.

\begin{tabular}{ccccccc}
\hline $\begin{array}{c}\text { Expected } \\
\text { Confidence } \\
(\%)\end{array}$ & $\begin{array}{c}\text { Confidence } \\
(\%)\end{array}$ & $\begin{array}{c}\text { Support } \\
(\%)\end{array}$ & Lift & Transações & Regra & ID da Regra \\
\hline 11,14 & 27,24 & 0,86 & 2,44 & 1483,0 & $10 \& 3==>6$ & 2 \\
3,17 & 7,75 & 0,86 & 2,44 & 1483,0 & $6==>10 \& 3$ & 1 \\
13,54 & 32,67 & 0,97 & 2,41 & 1659,0 & $5 \& 3==>4$ & 4 \\
2,96 & 7,14 & 0,97 & 2,41 & 1659,0 & $4==>5 \& 3$ & 3 \\
13,54 & 32,17 & 0,93 & 2,38 & 1594,0 & $5 \& 2==>4$ & 6 \\
2,89 & 6,86 & 0,93 & 2,38 & 1594,0 & $4==>5 \& 2$ & 7 \\
6,17 & 14,26 & 0,97 & 2,31 & 1659,0 & $5==>4 \& 3$ & 8 \\
6,77 & 15,67 & 0,97 & 2,31 & 1659,0 & $4 \& 3==>5$ & 10 \\
13,54 & 30,44 & 1,32 & 2,25 & 2272,0 & $6 \& 2==>4$ & 4 \\
4,35 & 9,78 & 1,32 & 2,25 & 2272,0 & $4==>6 \& 2$ & 9 \\
\hline
\end{tabular}

Tabela 18: Regras de maior lift-planos de pagamento por mês

A Tabela 19 apresenta as dez regras de maior support, resultantes da AA sobre os dados de planos de pagamento. Observa-se que as regras com altos valores atribuídos ao support, demonstram o lift baixo e próximo a 1. Conclui-se então, que muitas regras encontradas na análise não serão consideradas no modelo de segmentação.

\begin{tabular}{|c|c|c|c|c|c|c|}
\hline $\begin{array}{c}\text { Expected } \\
\text { Confidence } \\
(\%)\end{array}$ & $\begin{array}{c}\text { Confidence } \\
(\%)\end{array}$ & $\begin{array}{c}\text { Support } \\
(\%)\end{array}$ & Lift & Transações & Regra & ID da Regra \\
\hline 82,35 & 80,92 & 27,22 & 0,98 & 46720 & $2==>1$ & 171 \\
\hline 33,63 & 33,05 & 27,22 & 0,98 & 46720 & $1==>2$ & 172 \\
\hline 82,35 & 78,70 & 24,53 & 0,96 & 42115 & $3==>1$ & 175 \\
\hline 31,17 & 29,79 & 24,53 & 0,96 & 42115 & $1==>3$ & 176 \\
\hline 33,63 & 43,84 & 13,67 & 1,30 & 23460 & $3==>2$ & 128 \\
\hline 31,17 & 40,63 & 13,67 & 1,30 & 23460 & $2==>3$ & 129 \\
\hline 33,63 & 49,00 & 12,02 & 1,46 & 20638 & $3 \& 1==>2$ & 107 \\
\hline 24,53 & 35,74 & 12,02 & 1,46 & 20638 & $2==>3 \& 1$ & 106 \\
\hline 27,22 & 38,57 & 12,02 & 1,42 & 20638 & $3==>2 \& 1$ & 110 \\
\hline 31,17 & 44,17 & 12,02 & 1,42 & 20638 & $2 \& 1==>3$ & 111 \\
\hline
\end{tabular}

Tabela 19: Regras de maior support-planos de pagamento por mês

5.2.4 Análise de associação das cidades onde ocorreram as compras mensais

A Figura 17 apresenta o grafo de associação para a análise das cidades onde ocorreram as compras dos clientes a cada mês. Observa-se a concentração das transações entre Salvador e Recife, seguido por São Paulo e Natal, e depois por João Pessoa, Maceió, Jaboatão dos Guararapes, Curitiba, Fortaleza, Porto Alegre e Rio de Janeiro. Esta concentração nas grandes cidades praticamente coincide com a concentração dos clientes nos estados, demonstrada na Tabela 6 e é devida a condição inicial da distribuição geográfica da amostra de clientes. 
Observa-se que as associações entre estas cidades são fracas ou inexistentes, por não apresentar ou apresentar setas azuis e estreitas entre elas. No entanto, encontram-se associações fortes destas cidades com outras cidades bastante próximas, ilustradas pelas setas largas e vermelhas.

Contudo, mesmo que não tenha a associação muito forte, destaca-se a cidade do Rio de Janeiro.

A associação do Rio de Janeiro com Salvador, Recife ou São Paulo é de repulsão, pois apresenta lift inferior a 1, como apresentado na quarta coluna da Tabela 20.

A Tabela 20 mostra os parâmetros de associação entre o Rio de Janeiro e as demais cidades. Nota-se que a expectativa de que um cliente qualquer tenha uma transação em São Paulo é de 5,92\%, 12,02\% para Salvador e 12,62\% para Recife. Dado um cliente com transações no Rio de Janeiro, estas expectativas caem para $5,36 \%, 7,55 \%$ e $7,77 \%$ respectivamente.

\begin{tabular}{|c|c|c|c|c|c|c|}
\hline $\begin{array}{c}\text { Expected } \\
\text { Confidence } \\
(\%)\end{array}$ & $\begin{array}{c}\text { Confidence } \\
(\%)\end{array}$ & $\begin{array}{c}\text { Support } \\
(\%)\end{array}$ & Lift & Transações & Regra & ID da Regra \\
\hline 5,92 & 5,36 & 0,13 & 0,91 & 227,00 & $\begin{array}{c}\text { Rio de Janeiro ==> } \\
\text { São Paulo }\end{array}$ & 63 \\
\hline 12,02 & 7,55 & 0,19 & 0,63 & 320,00 & $\begin{array}{c}\text { Rio de Janeiro }==> \\
\text { Salvador }\end{array}$ & 65 \\
\hline 12,62 & 7,77 & 0,19 & 0,62 & 329,00 & $\begin{array}{c}\text { Rio de Janeiro }==> \\
\text { Recife }\end{array}$ & 66 \\
\hline
\end{tabular}

Tabela 20: Regras de associação do Rio de Janeiro 

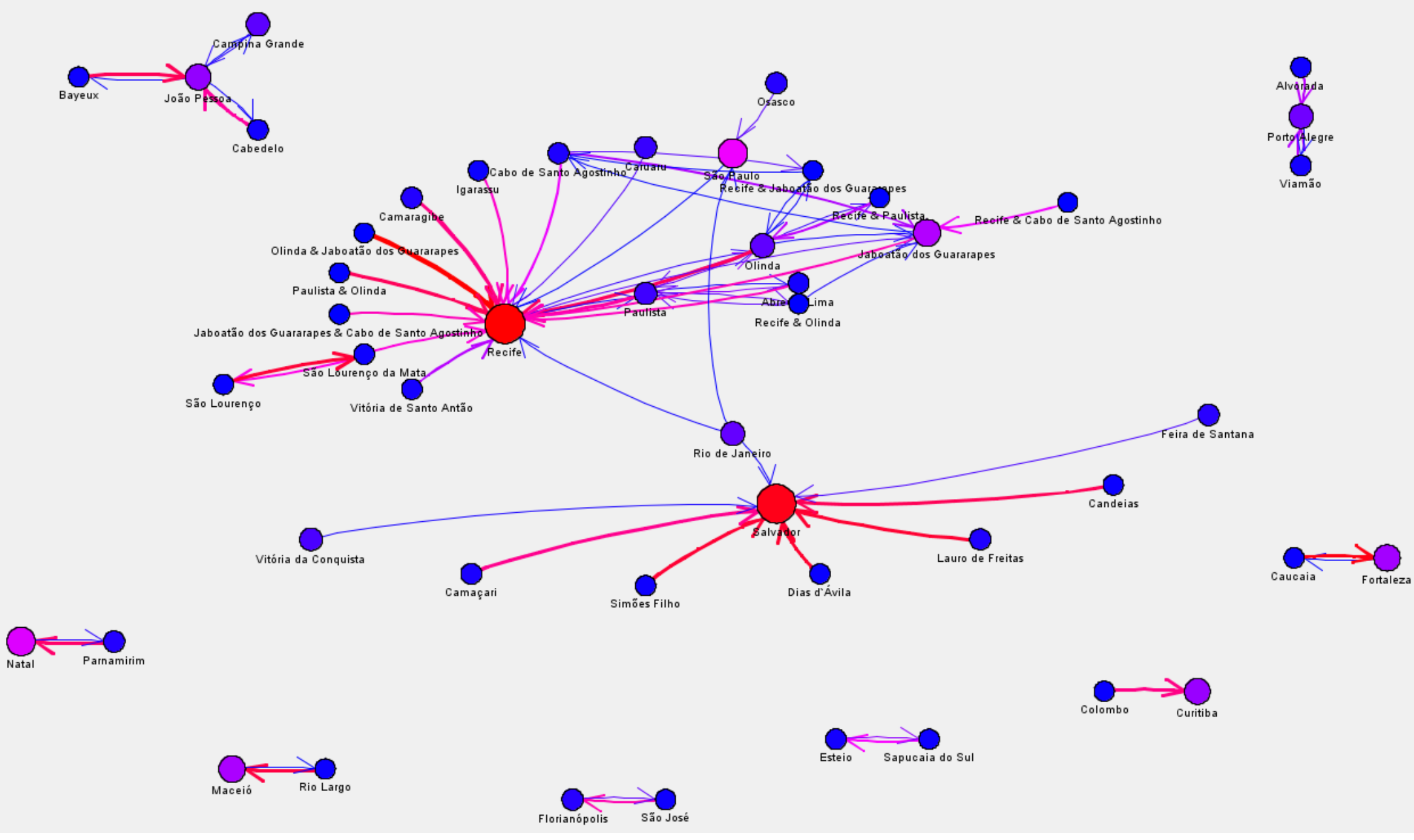

Figura 17: Grafo de associações das cidades por mês 
O Gráfico 8 apresenta a dispersão entre support e confidence das cem primeiras regras de associação de cidades. Support de $0,55 \%$ indica que apenas esta proporção de faturas contém tal combinação de cidades onde o cartão foi utilizado. No entanto, como o confidence é de $65 \%$, conclui-se que $65 \%$ das faturas cujas consignações apontarem transações realizadas em Camaragibe, constarão também transações realizadas em Recife.

As cidades Recife e Camaragibe se distanciam em aproximadamente 14 quilômetros e por ser a capital e mais desenvolvida, Recife atrai moradores de Camaragibe para o comércio. Entretanto, o contrário não ocorre.

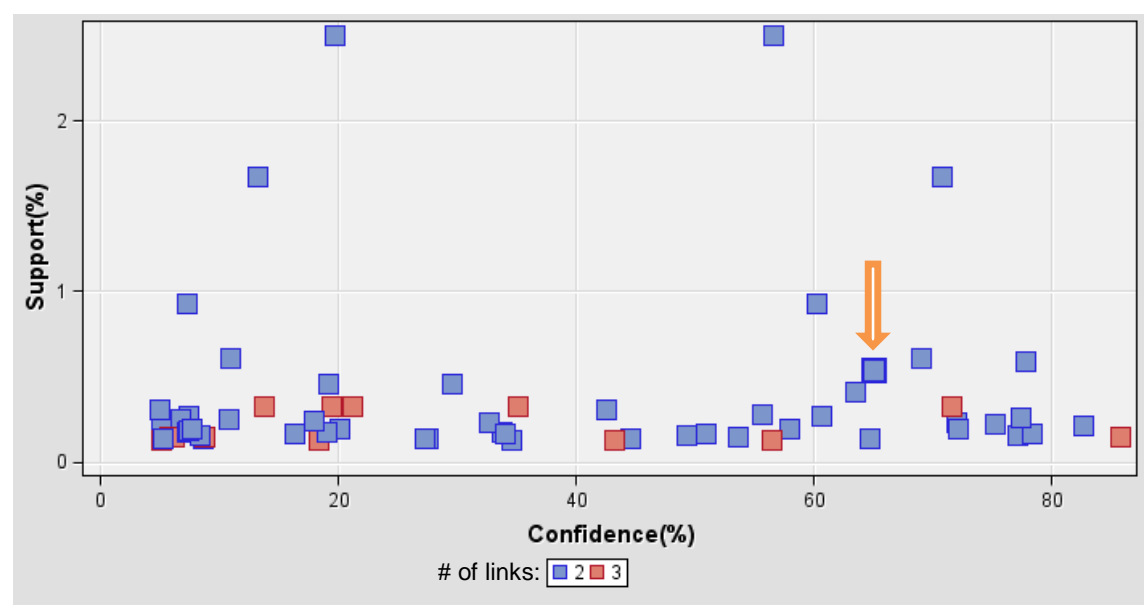

Gráfico 8: Support versus confidence para as primeiras regras de associação de cidades por mês

O Gráfico 9 apresenta o confidence, expected confidence, lift e support. Observa-se em destaque a regra 43 . O expected confidence (12,62\%) sinaliza que a expectativa de ter uma transação proveniente de Recife em qualquer fatura é de 12,62\%; mas dado que esta fatura possui transações originadas em Camaragibe, esta expectativa passará a traduzir $65 \%$ (confidence). 


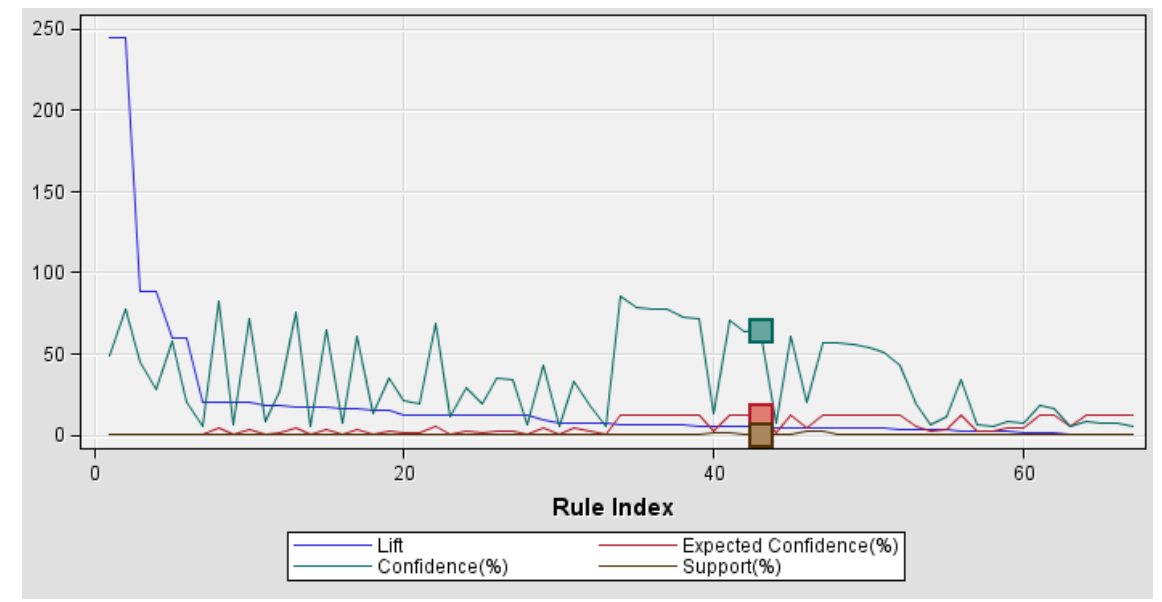

Gráfico 9: Lift, confidence, expected confidence e support de cidades por mês

A divisão do confidence pelo expected confidence resulta no lift de 5,15. A leitura do Gráfico 9 nos indica que pouco mais de 60 regras de associação foram encontradas na análise. Entretanto o valor elevado do lift de boa parte destas regras fará com que sejam relevantes no modelo de segmentação de clientes pelo perfil de consumo.

A Figura 18 apresenta todas as regras dispostas entre seus antecedentes e consequentes, percebe-se a escassez de regras provenientes desta análise.

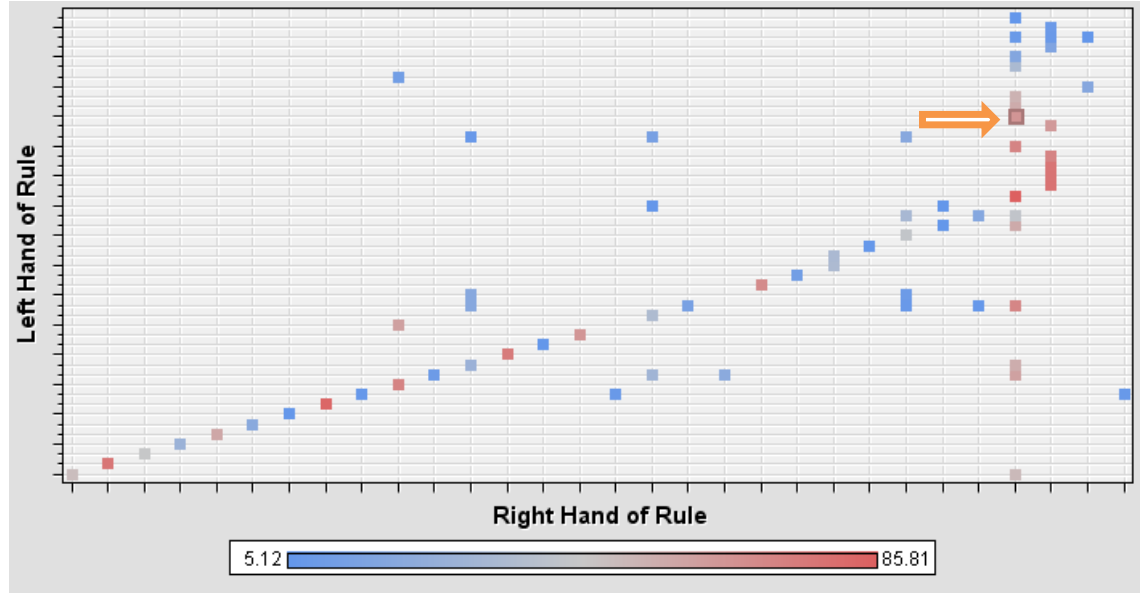

Figura 18: Matriz de regras de associação de cidades por mês

As análises referentes aos Gráficos 8, 9 e Figura 18, são ratificadas por exemplos encontrados nas Tabelas 21 e 22.

A Tabela 21 apresenta as 10 primeiras regras ordenadas pelo lift. 


\begin{tabular}{|c|c|c|c|c|c|c|}
\hline $\begin{array}{c}\text { Expected } \\
\text { Confidence } \\
(\%)\end{array}$ & $\begin{array}{c}\text { Confidence } \\
(\%)\end{array}$ & $\begin{array}{c}\text { Support } \\
(\%)\end{array}$ & Lift & Transações & Regra & ID da Regra \\
\hline 0,20 & 49,35 & 0,16 & 244,86 & 267,00 & $\begin{array}{l}\text { São Lourenço da } \\
\text { Mata ==> São } \\
\text { Lourenço }\end{array}$ & 1 \\
\hline 0,32 & 77,17 & 0,16 & 244,86 & 267,00 & $\begin{array}{c}\text { São Lourenço ==> } \\
\text { São Lourenço da } \\
\text { Mata }\end{array}$ & 2 \\
\hline 0,50 & 44,67 & 0,14 & 88,86 & 239,00 & $\begin{array}{c}\text { Sapucaia do Sul }==> \\
\text { Esteio }\end{array}$ & 3 \\
\hline 0,31 & 27,69 & 0,14 & 88,86 & 239,00 & $\begin{array}{c}\text { Esteio }==>\text { Sapucaia } \\
\text { do Sul }\end{array}$ & 4 \\
\hline 0,96 & 58,06 & 0,20 & 60,26 & 335,00 & $\begin{array}{l}\text { São José }==> \\
\text { Florianópolis }\end{array}$ & 5 \\
\hline 0,34 & 20,25 & 0,20 & 60,26 & 335,00 & $\begin{array}{c}\text { Florianópolis }==> \\
\text { São José }\end{array}$ & 6 \\
\hline 0,25 & 5,20 & 0,21 & 20,52 & 360,00 & $\begin{array}{c}\text { Fortaleza }==> \\
\text { Caucaia }\end{array}$ & 7 \\
\hline 4,03 & 82,76 & 0,21 & 20,52 & 360,00 & $\begin{array}{c}\text { Caucaia }==> \\
\text { Fortaleza }\end{array}$ & 8 \\
\hline 0,31 & 6,20 & 0,23 & 19,78 & 388,00 & $\begin{array}{c}\text { João Pessoa }==> \\
\text { Bayeux }\end{array}$ & 9 \\
\hline 3,65 & 72,12 & 0,23 & 19,78 & 388,00 & $\begin{array}{c}\text { Bayeux }==>\text { João } \\
\text { Pessoa }\end{array}$ & 10 \\
\hline
\end{tabular}

Tabela 21: Regras de maior lift - cidades por mês

A Tabela 22 apresenta as 10 primeiras regras segundo o support.

\begin{tabular}{|c|c|c|c|c|c|c|}
\hline $\begin{array}{c}\text { Expected } \\
\text { Confidence } \\
(\%)\end{array}$ & $\begin{array}{c}\text { Confidence } \\
(\%)\end{array}$ & $\begin{array}{l}\text { Support } \\
(\%)\end{array}$ & Lift & Transações & Regra & ID da Regra \\
\hline 4,41 & 19,84 & 2,50 & 4,49 & 4299,0 & $\begin{array}{c}\text { Recife }==>\text { Jaboatão } \\
\text { dos Guararapes }\end{array}$ & 46 \\
\hline 12,62 & 56,73 & 2,50 & 4,49 & 4299,0 & $\begin{array}{c}\text { Jaboatão dos } \\
\text { Guararapes ==> } \\
\text { Recife }\end{array}$ & 47 \\
\hline 2,36 & 13,25 & 1,67 & 5,61 & 2872,0 & Recife $==>$ Olinda & 40 \\
\hline 12,62 & 70,88 & 1,67 & 5,61 & 2872,0 & Olinda $==>$ Recife & 41 \\
\hline 1,54 & 7,34 & 0,93 & 4,78 & 1590,0 & Recife $==>$ Paulista & 44 \\
\hline 12,62 & 60,30 & 0,93 & 4,78 & 1590,0 & Paulista $==>$ Recife & 45 \\
\hline 5,47 & 69,07 & 0,60 & 12,63 & 1036,0 & $\begin{array}{c}\text { Parnamirim } \\
\text { Natal }\end{array}==>$ & 22 \\
\hline 0,87 & 11,04 & 0,60 & 12,63 & 1036,0 & $\begin{array}{c}\text { Natal }==> \\
\text { Parnamirim }\end{array}$ & 23 \\
\hline 12,02 & 77,97 & 0,59 & 6,49 & 1012,0 & $\begin{array}{c}\text { Lauro de Freitas ==> } \\
\text { Salvador }\end{array}$ & 36 \\
\hline 12,62 & 65,00 & 0,55 & 5,15 & 936,00 & $\begin{array}{c}\text { Camaragibe }==> \\
\text { Recife }\end{array}$ & 43 \\
\hline
\end{tabular}

Tabela 22: Regras de maior support-cidades por mês

\subsubsection{Análise de associação das famílias de produtos nas compras anuais}

A Figura 19 apresenta o resultado da aplicação da AA às transações realizadas pelos clientes durante período estudado (12 meses). 
$\mathrm{Na}$ análise das transações realizadas pelos 26.767 clientes, excluindo-se as faturas da amostra de validação, percebe-se que as associações são mais frequentes, ou seja, possuem um maior indicador de support.

Observa-se que as associações destacadas na análise mensal, por vezes serão substituídas por outras mais relevantes, o que será indicativo que os comportamentos se repetem entre as pessoas; contudo em períodos distintos.

Tanto na análise de transações mensais (Seção 5.2.1) quanto na Figura 19 (análise de transações anuais), duas constelações são apresentadas.

$\mathrm{Na}$ visão mensal, a maior constelação contém regras de associação entre diversão, veículos, alimentos e serviços; a menor destaca posto de gasolina.

$\mathrm{Na}$ visão anual, a maior constelação contém regras entre as famílias: geral, diversão, perfumaria, joalheria e livraria, indiretamente associadas a veículos, postos de gasolina e alimentos; a menor possui o centro em viagens.

Esta leitura mostra que embora dentro de um mês não se apresente muitas associações que possam oferecer previsão de gasto com viagem dos clientes, ao observar uma sequência maior de transações dos clientes, tem-se bons indicadores de expectativa de consumo desta família de produtos.

Os conjuntos de regras são diferentes ao observar um mês de compras de um cliente comparando a um ano de compras deste mesmo cliente. Considerando que em um ano, o cliente passa normalmente por um período curto de férias e um período longo de trabalho, encontra-se consumos similares entre diferentes clientes ao longo do ano, de acordo com os momentos em que estiveram ou não em férias. Como esta análise independe da ordem das transações para a criação das regras, ao se avaliar o período de 12 meses de todos os clientes as associações de consumo são expostas, mesmo que tenham ocorrido em períodos distribuídos durante $o$ ano. 


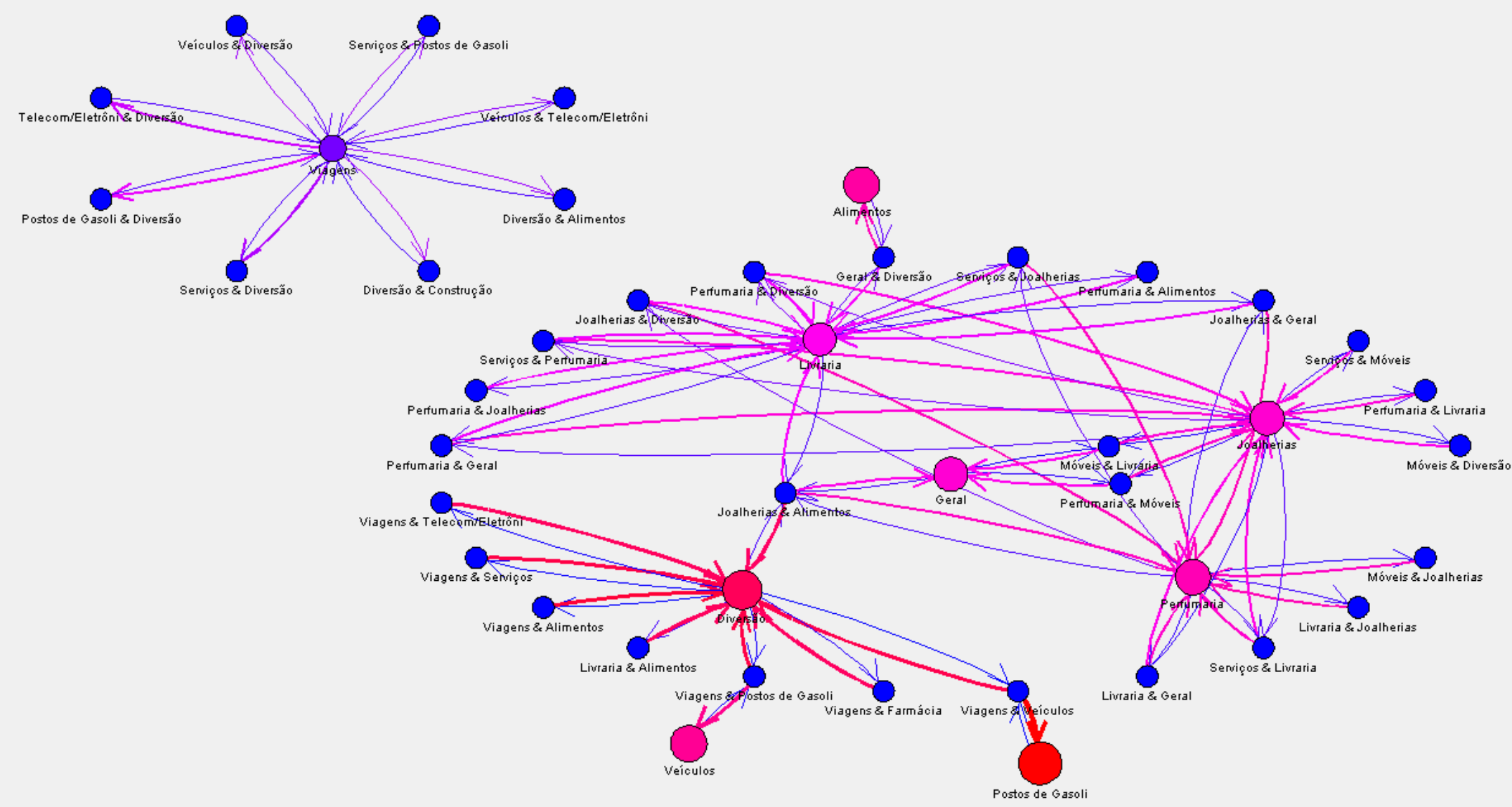

Figura 19: Grafo de associações de famílias de produtos por ano 
A regra destacada nos Gráficos 10, 11 e Figura 20 é a de número 52 (Livraria \& Alimentos $==>$ Diversão) que embora associe três famílias de produtos, consumidas durante o ano, tem elevado support de 5,47\% e acusa um lift de 2,29. A Tabela 23 indica todas as medidas dos indicadores desta regra.

\begin{tabular}{ccccccc}
\hline $\begin{array}{c}\text { Expected } \\
\text { Confidence } \\
(\%)\end{array}$ & $\begin{array}{c}\text { Confidence } \\
(\%)\end{array}$ & $\begin{array}{c}\text { Support } \\
(\%)\end{array}$ & Lift & Transações & Regra & ID da Regra \\
\hline 30,04 & 68,88 & 5,47 & 2,29 & 1465,0 & $\begin{array}{c}\text { Livraria \& Alimentos } \\
==>\text { Diversão }\end{array}$ & 52 \\
\hline
\end{tabular}

Tabela 23: Regra 52 de família de produtos por ano

Analisando-se apenas esta regra como oportunidade para a segmentação dos clientes de cartão de crédito, pode-se imaginar a seguinte situação:

Um parque de diversões aceita cartão de crédito no pagamento de seus ingressos e oferece uma condição especial de preço aos clientes da carteira de cartões de crédito analisada neste estudo.

É conhecida a informação de que 30,04\% dos clientes que utilizarem o cartão ao longo do ano terão algum gasto com este tipo de produto (8.041 clientes), mas para atingir todos estes clientes, seria necessário comunicar 38.357 clientes durante o ano, pois até o encerramento do ano, não se sabe se o cliente irá ou não utilizar o cartão. Supondo-se um retorno de comunicação de 100\%, ter-se-ia 4,77 comunicações por adesão.

O Gráfico 10 apresenta a dispersão entre support e confidence das cem primeiras regras de associação famílias de produtos, na visão anual de transações. 


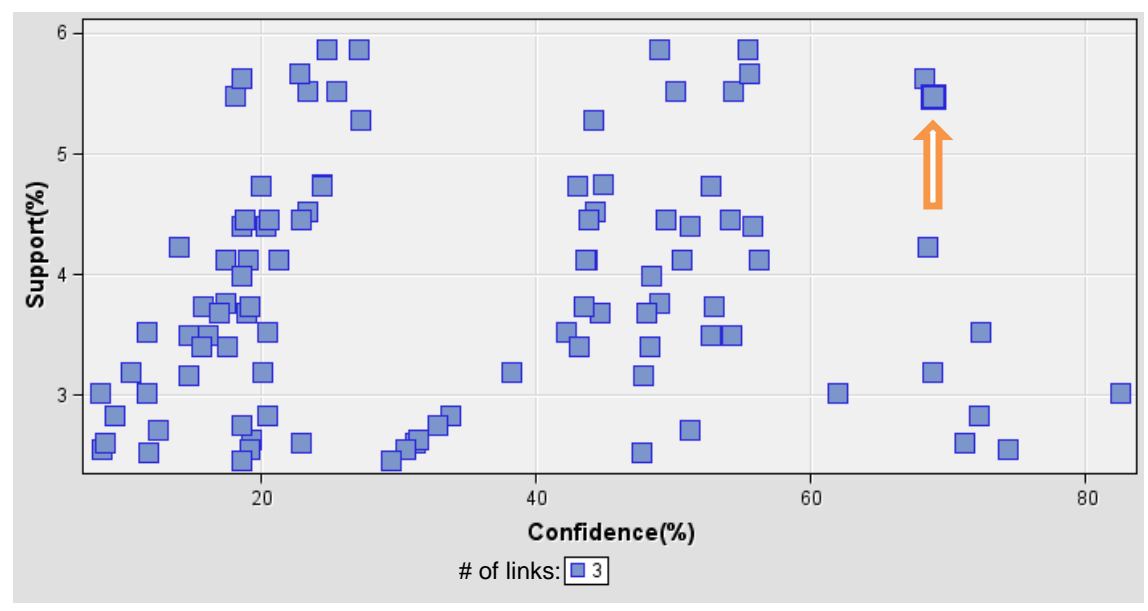

Gráfico 10: Support versus confidence para as cem primeiras regras de associação de famílias de produtos por ano

Utilizando a regra de associação para segmentar o público desta oferta, percebe-se que como $5,47 \%$ dos clientes possuem as transações que atendem a regra 52 , com confidence de $68,88 \%$. Esses dados nos permitem concluir que 7,94\% dos clientes preenchem a condição antecedente da regra (Livraria \& Alimentos), resultando em 2.125 clientes que viriam a ser sensibilizados pela oferta com o potencial de 1.465 clientes consumirem o produto; ou seja, supondo-se os mesmos $100 \%$ de retorno sobre a comunicação, ter-se-ia 1,45 comunicações por adesão.

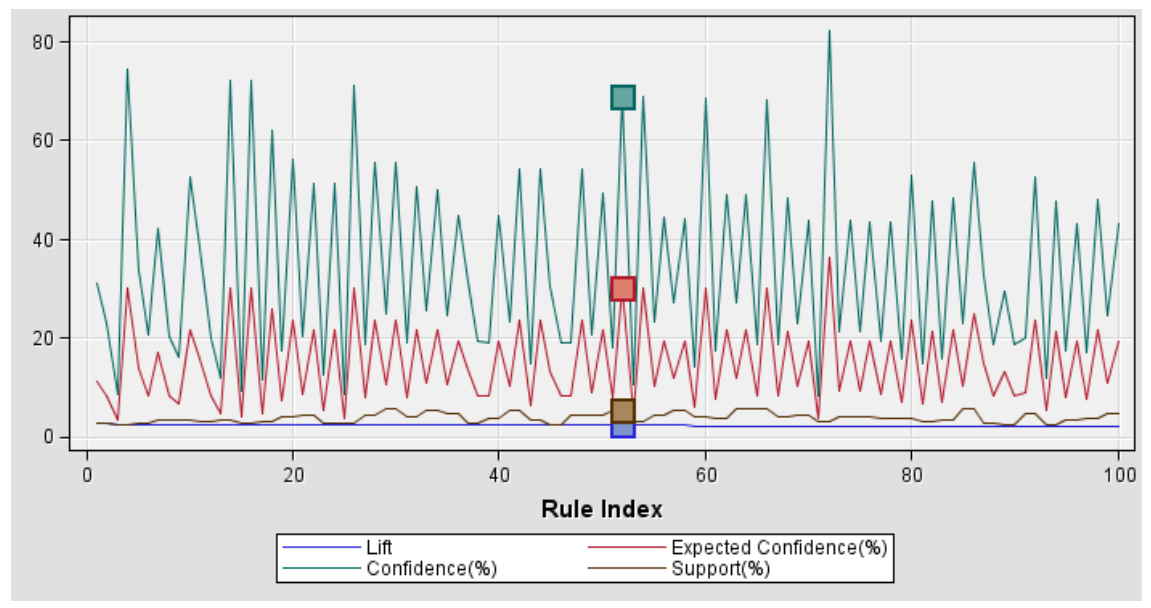

Gráfico 11: Lift, confidence, expected confidence e support de famílias de produtos por ano

A grande vantagem é que embora se tenha reduzido de 8.041 clientes potenciais para 1.465 clientes ( $18 \%$ do potencial), a comunicação teve seu custo reduzido para $5,5 \%$ do custo de comunicação (de 38.357 para 2.125 clientes sensibilizados). Considerando-se a redução do custo de comunicação, a oferta 
poderá ser mais atrativa, para gerar uma diferenciação expressiva para o retorno desta ação. Além disso, os clientes não segmentados para a recepção desta comunicação estarão segmentados em alguma outra regra, o que poderá favorecer maior eficiência para a seleção de ofertas comunicadas à carteira de clientes de cartões de crédito.

A Figura 20 apresenta a distribuição das regras encontradas na análise entre antecedentes e consequentes, bem como o confidence de cada associação por meio da escala de cores.

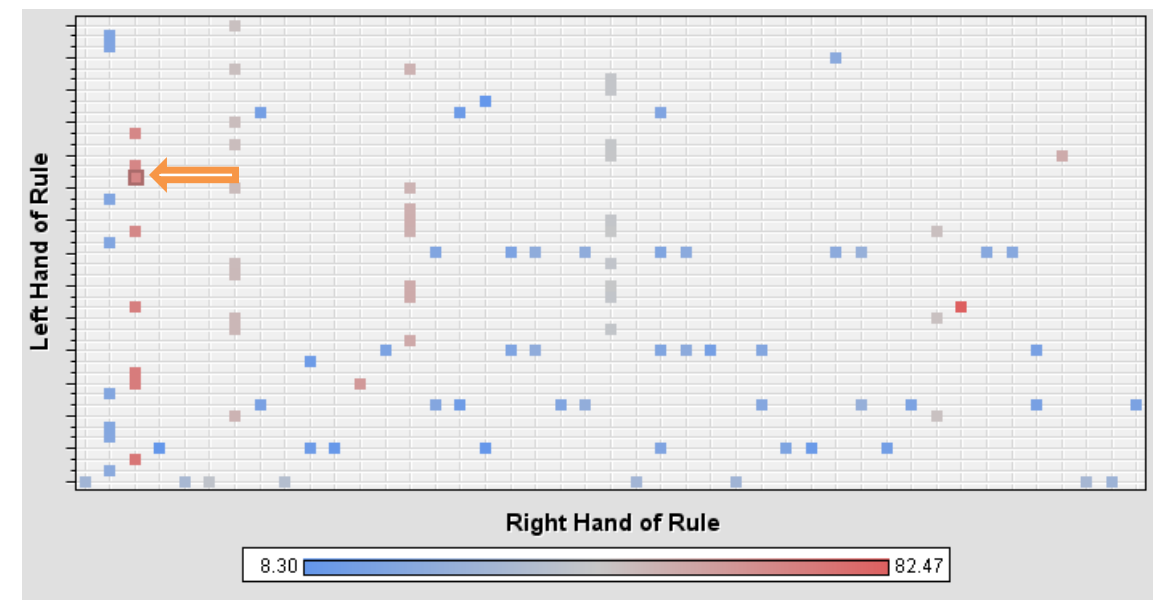

Figura 20: Matriz de regras de associação de família de produtos por ano

Interpretação semelhante à realizada a partir da regra 52 tem-se com todas as regras e a relação entre o ganho de eficiência na comunicação versus a vantagem comercial obtida pelo cliente do cartão de crédito servirá de guia para a obtenção do modelo de segmentação a ser utilizado na base de clientes.

$\mathrm{Na}$ Tabela 24, observa-se que entre as regras de alto lift, o support varia entre $3,53 \%$ e $2,55 \%$. 


\begin{tabular}{|c|c|c|c|c|c|c|}
\hline $\begin{array}{c}\text { Expected } \\
\text { Confidence } \\
(\%)\end{array}$ & $\begin{array}{c}\text { Confidence } \\
(\%)\end{array}$ & $\begin{array}{c}\text { Support } \\
(\%)\end{array}$ & Lift & Transações & Regra & ID da Regra \\
\hline 11,35 & 31,28 & 2,61 & 2,75 & 699,00 & $\begin{array}{c}\text { Viagens }==> \\
\text { Veículos \& Diversão }\end{array}$ & 1 \\
\hline 8,35 & 23,00 & 2,61 & 2,75 & 699,00 & $\begin{array}{l}\text { Veículos \& Diversão } \\
==>\text { Viagens }\end{array}$ & 2 \\
\hline 30,04 & 74,40 & 2,55 & 2,48 & 683,00 & $\begin{array}{l}\text { Viagens \& Alimentos } \\
\text { ==> Diversão }\end{array}$ & 4 \\
\hline 3,43 & 8,49 & 2,55 & 2,48 & 683,00 & $\begin{array}{c}\text { Diversão }==> \\
\text { Viagens \& Alimentos }\end{array}$ & 3 \\
\hline 13,77 & 33,87 & 2,83 & 2,46 & 757,00 & $\begin{array}{l}\text { Viagens }==> \\
\text { Serviços \& Diversão }\end{array}$ & 5 \\
\hline 8,35 & 20,54 & 2,83 & 2,46 & 757,00 & $\begin{array}{l}\text { Serviços \& Diversão } \\
\quad==>\text { Viagens }\end{array}$ & 6 \\
\hline 17,24 & 42,24 & 3,53 & 2,45 & 944,00 & $\begin{array}{c}\text { Viagens }==>\text { Postos } \\
\text { de Gasolina \& } \\
\text { Diversão }\end{array}$ & 7 \\
\hline 8,35 & 20,46 & 3,53 & 2,45 & 944,00 & $\begin{array}{c}\text { Postos de Gasolina } \\
\text { \& Diversão ==> } \\
\text { Viagens }\end{array}$ & 8 \\
\hline 21,58 & 52,80 & 3,49 & 2,45 & 935,00 & $\begin{array}{l}\text { Perfumaria \& Móveis } \\
==>\text { Joalherias }\end{array}$ & 10 \\
\hline 6,62 & 16,19 & 3,49 & 2,45 & 935,00 & $\begin{array}{c}\text { Joalherias }==> \\
\text { Perfumaria \& Móveis }\end{array}$ & 9 \\
\hline
\end{tabular}

Tabela 24: Regras de maior lift - família de produtos por ano

Observa-se na Tabela 25 que as regras com altos valores atribuídos ao support, demonstram o lift baixo e próximo a 1. Conclui-se então, que muitas regras encontradas na análise não serão consideradas no modelo de segmentação.

\begin{tabular}{|c|c|c|c|c|c|c|}
\hline $\begin{array}{c}\text { Expected } \\
\text { Confidence } \\
(\%)\end{array}$ & $\begin{array}{c}\text { Confidence } \\
(\%)\end{array}$ & $\begin{array}{c}\text { Support } \\
(\%)\end{array}$ & Lift & Transações & Regra & ID da Regra \\
\hline 81,23 & 82,25 & 51,47 & 1,01 & 13778 & $\begin{array}{l}\text { Vestuário }==> \\
\text { Supermercado }\end{array}$ & 3956 \\
\hline 62,58 & 63,37 & 51,47 & 1,01 & 13778 & $\begin{array}{c}\text { Supermercado } \\
\text { Vestuário }\end{array}==>$ & 3957 \\
\hline 81,23 & 83,14 & 46,68 & 1,02 & 12494 & $\begin{array}{l}\text { Lojas em Geral ==> } \\
\text { Supermercado }\end{array}$ & 3955 \\
\hline 56,14 & 57,46 & 46,68 & 1,02 & 12494 & $\begin{array}{c}\text { Supermercado }==> \\
\text { Lojas em Geral }\end{array}$ & 3954 \\
\hline 81,23 & 85,27 & 45,57 & 1,05 & 12199 & $\begin{array}{l}\text { Farmácia }==> \\
\text { Supermercado }\end{array}$ & 3945 \\
\hline 53,45 & 56,11 & 45,57 & 1,05 & 12199 & $\begin{array}{c}\text { Supermercado }==> \\
\text { Farmácia }\end{array}$ & 3944 \\
\hline 62,58 & 80,58 & 45,24 & 1,29 & 12110 & $\begin{array}{c}\text { Lojas em Geral }==> \\
\text { Vestuário }\end{array}$ & 3655 \\
\hline 56,14 & 72,29 & 45,24 & 1,29 & 12110 & $\begin{array}{c}\text { Vestuário }==>\text { Lojas } \\
\text { em Geral }\end{array}$ & 3654 \\
\hline 62,58 & 79,60 & 42,54 & 1,27 & 11388 & $\begin{array}{c}\text { Farmácia }==> \\
\text { Vestuário }\end{array}$ & 3669 \\
\hline 53,45 & 67,98 & 42,54 & 1,27 & 11388 & $\begin{array}{c}\text { Vestuário ==> } \\
\text { Farmácia }\end{array}$ & 3668 \\
\hline
\end{tabular}

Tabela 25: Regras de maior support - família de produtos por ano

5.2.6 Análise de associação dos produtos nas compras anuais

De acordo com a exposição da mesma análise comparativa entre as associações das compras mensais e anuais, percebe-se que ao analisarmos 
anualmente as cem primeiras associações na ordem do maior para o menor lift, algumas pequenas constelações se apresentam. 


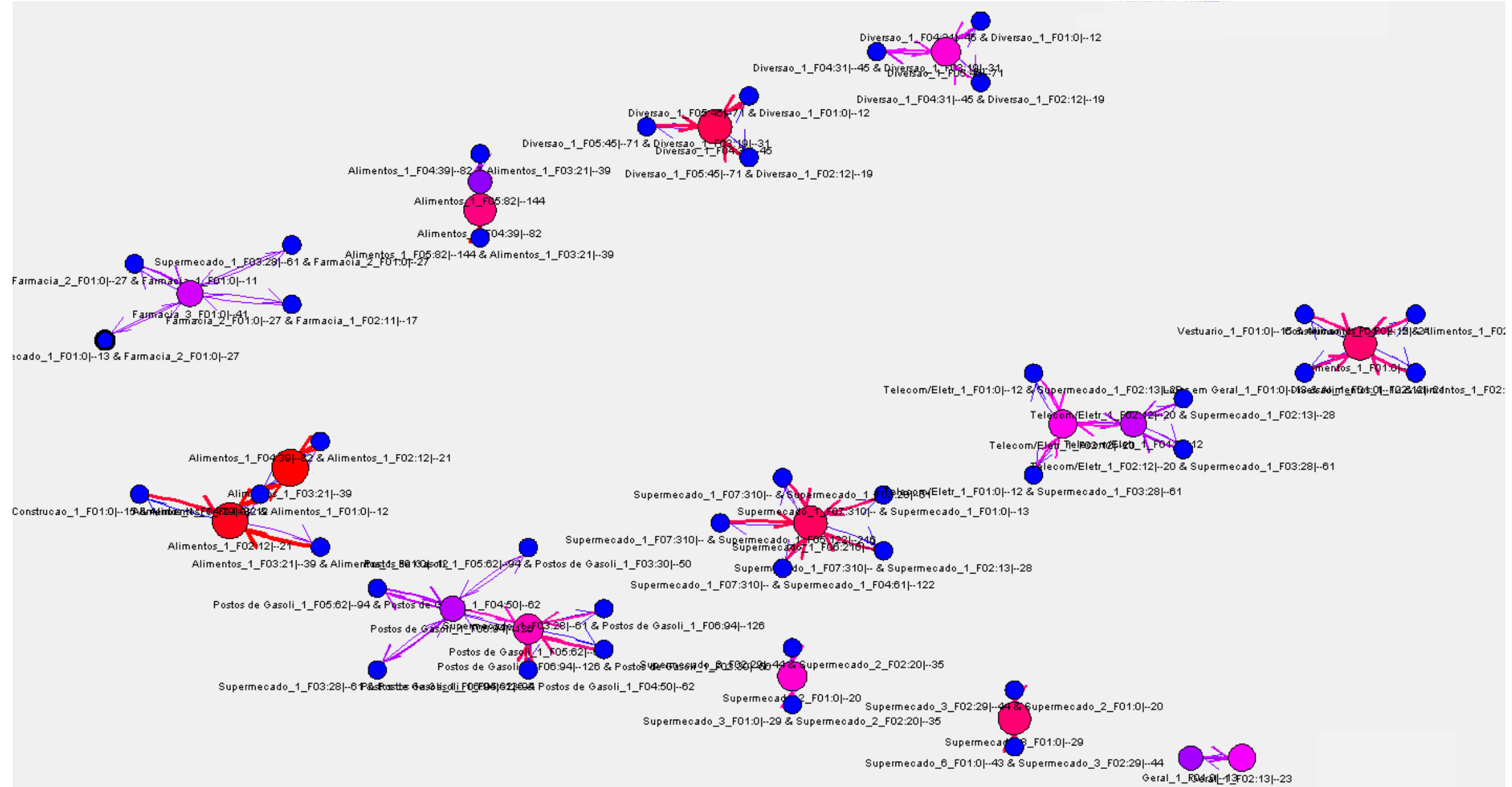

Figura 21: Grafo de associações de produtos por ano (parte1) 


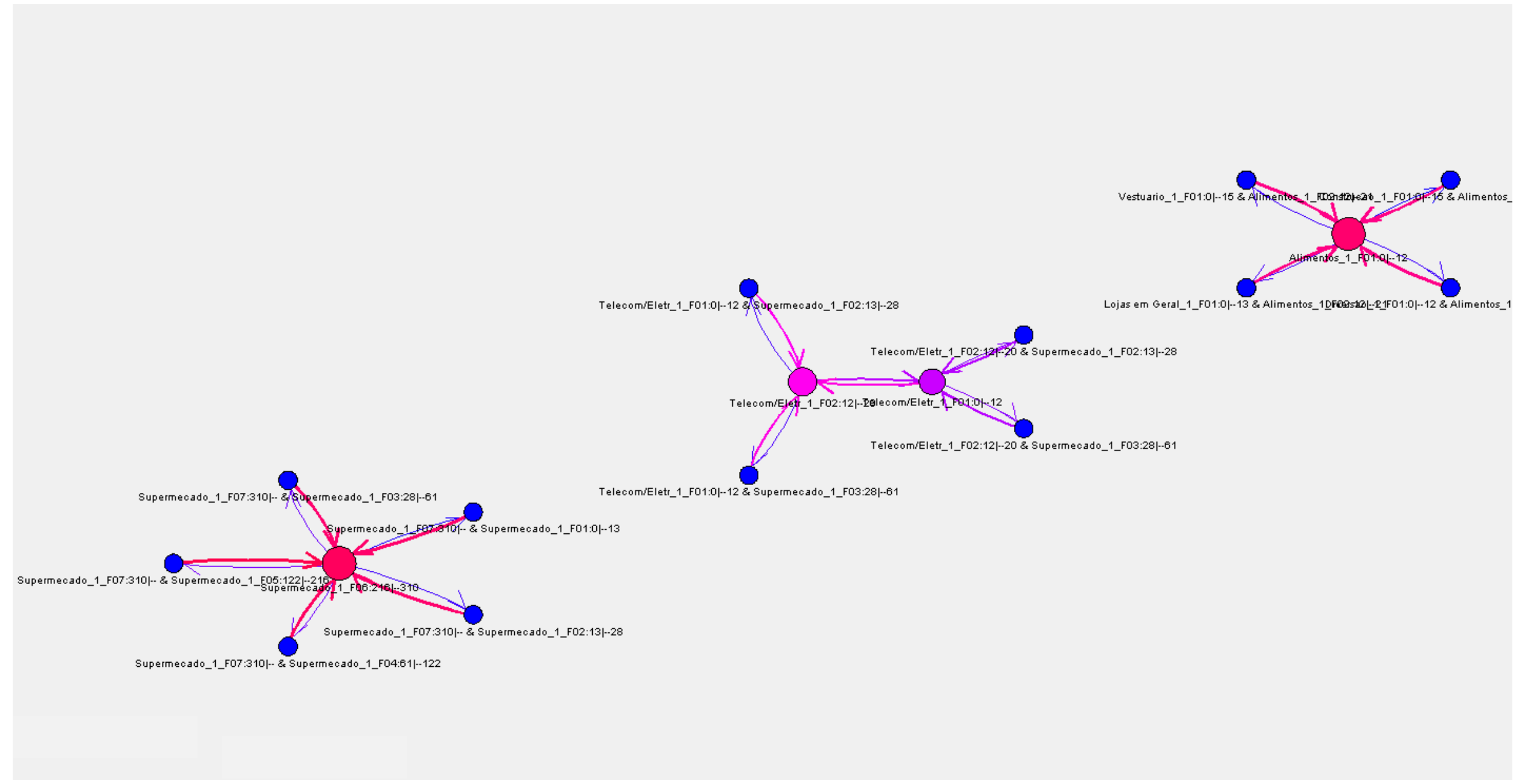

Figura 22: Grafo de associações de produtos por ano (parte 2) 
A regra de número 16 (Telecomunicações / Eletrônicos_1_F01:0|--12 \& Supermercado_1_F03:28|--61 ==> Telecomunicações / Eletrônicos_1_F02:12|--20), destacada nos Gráficos 12 e 13, a seguir, mostra a associação entre as aquisições de Produtos de Telecomunicações ou Eletrônicos de baixo valor (na primeira faixa de valores da família de produtos), juntamente com compras no Supermercado, na terceira faixa de valor (na terceira faixa de valores), que indicam o consumo da família de produtos Telecomunicações ou Eletrônicos em uma faixa mais elevada de valores (segunda faixa de valores da família de produtos).

Pode-se ilustrar esta associação com o consumo de uma recarga de celular de até $R \$ 12,00$, juntamente com uma compra de Supermercado quinzenal, para indicar uma nova compra de recarga de Celular no valor entre $R \$ 12,00$ e $R \$ 20,00$.

O Gráfico 12 apresenta a dispersão entre support e confidence das cem primeiras regras de associação de produtos na visão anual.

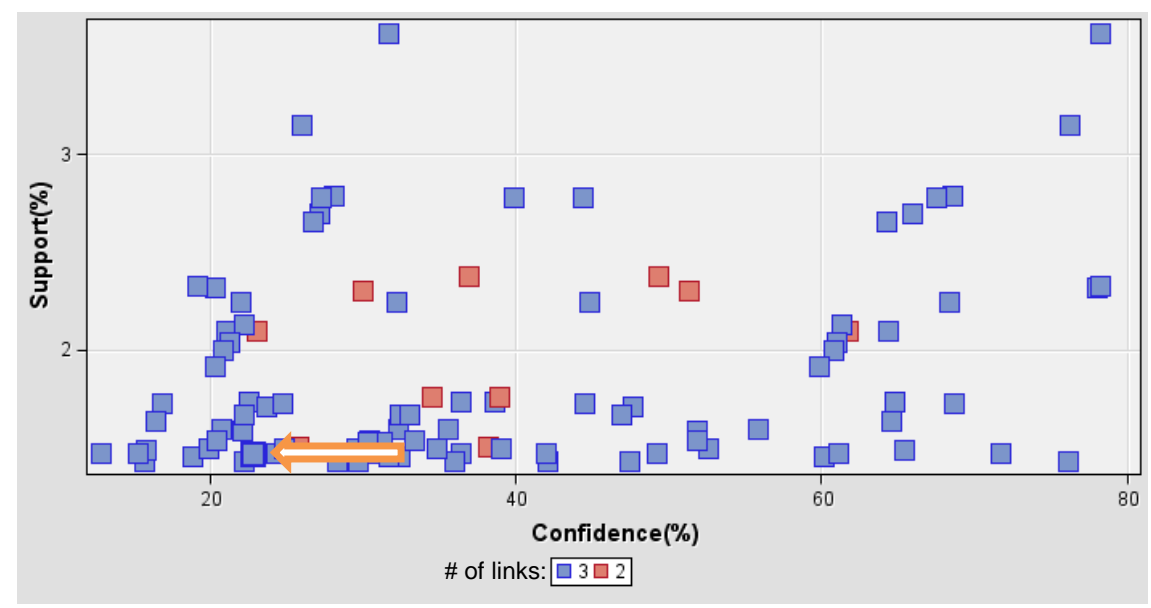

Gráfico 12: Support versus confidence para as cem primeiras regras de associação de produtos por ano

Percebe-se no Gráfico 13 que entre as cem primeiras regras de associação, apresentam-se altos valores para o lift, em média 6,9. Isto indica que uma quantidade muito grande de regras de associação, além das primeiras cem, será utilizada no modelo de segmentação. 


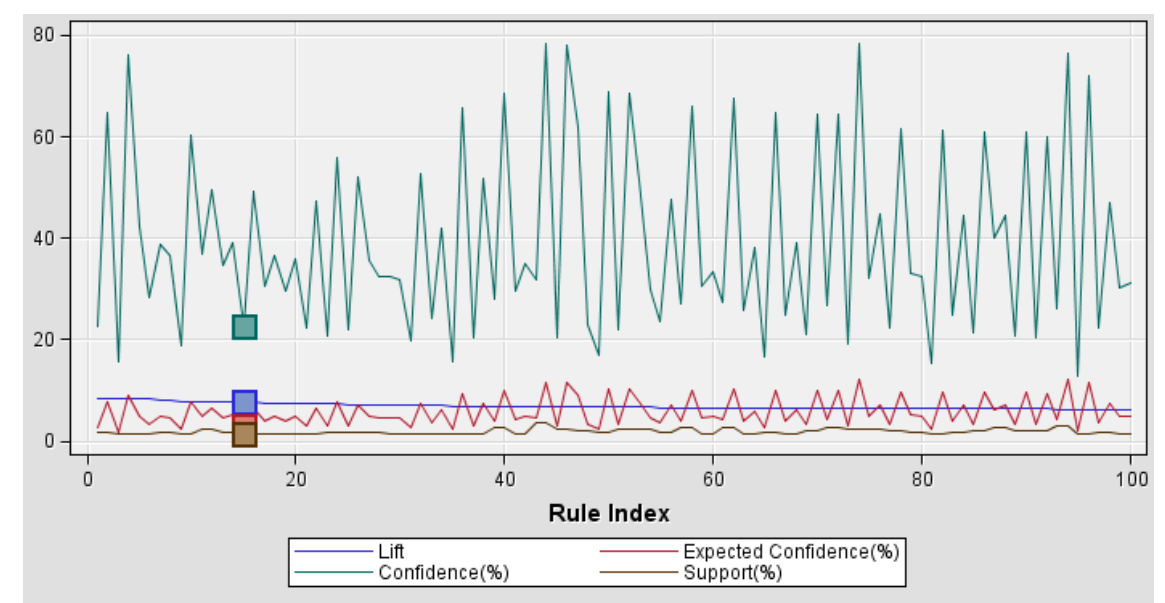

Gráfico 13: Lift, confidence, expected confidence e support de produtos por ano

A Figura 23 apresenta a distribuição das regras encontradas na análise entre antecedentes e consequentes, bem como o confidence de cada associação por meio da escala de cores.

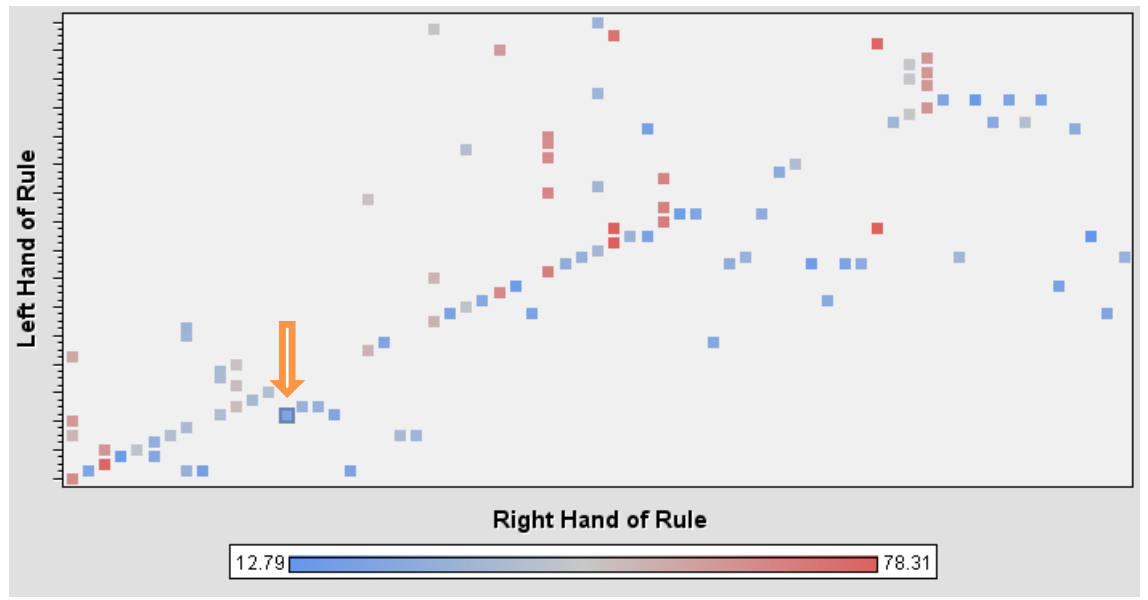

Figura 23: Matriz de regras de associação de produtos por ano

Notam-se na Tabela 26, regras com altos valores para o lift e support superior a $1 \%$, o que vem a ser um indicativo de que estas regras contribuirão com a eficiência do modelo de segmentação. 


\begin{tabular}{|c|c|c|c|c|c|c|}
\hline $\begin{array}{c}\text { Expected } \\
\text { Confidence } \\
(\%)\end{array}$ & $\begin{array}{c}\text { Confidence } \\
(\%)\end{array}$ & $\begin{array}{c}\text { Support } \\
(\%)\end{array}$ & Lift & Transações & Regra & $\begin{array}{l}\text { ID da } \\
\text { Regra }\end{array}$ \\
\hline 7,68 & 64,76 & 1,73 & 8,43 & 463,00 & $\begin{array}{c}\text { Postos de } \\
\text { Gasolina_1_F06:94|--126 \& } \\
\text { Postos de } \\
\text { Gasolina_1_F04:50|--62 } \\
==>\text { Postos de } \\
\text { Gasolina_1_F05:62|--94 }\end{array}$ & 2 \\
\hline 2,67 & 22,52 & 1,73 & 8,43 & 463,00 & $\begin{array}{c}\text { Postos de } \\
\text { Gasolina_1_F05:62|--94 } \\
==>\text { Postos de } \\
\text { Gasolina_1_F06:94|--126 \& } \\
\text { Postos de } \\
\text { Gasolina_1_F04:50|--62 }\end{array}$ & 1 \\
\hline 9,12 & 76,14 & 1,43 & 8,35 & 383,00 & $\begin{array}{l}\text { Alimentos_1_F05:82|--144 } \\
\text { \& Alimentos_1_F03:21|--39 } \\
==>\text { Alimentos_1_F04:39|-- } \\
82\end{array}$ & 4 \\
\hline 1,88 & 15,68 & 1,43 & 8,35 & 383,00 & $\begin{aligned} & \text { Alimentos_1_F04:39|--82 } \\
&==>\text { Alimentos_1_F05:82|-- } \\
& 144 \& \\
& \text { Alimentos_1_F03:21|--39 }\end{aligned}$ & 3 \\
\hline 5,05 & 42,09 & 1,43 & 8,33 & 383,00 & $\begin{array}{c}\text { Alimentos_1_F05:82|-144 } \\
==>\text { Alimentos_1_F04:39|-- } \\
82 \text { \& Alimentos_1_F03:21|- } \\
\text {-39 }\end{array}$ & 5 \\
\hline 3,40 & 28,33 & 1,43 & 8,33 & 383,00 & $\begin{array}{c}\text { Alimentos_1_F04:39|--82 \& } \\
\text { Alimentos_1_F03:21|--39 } \\
==>\text { Alimentos_1_F05:82|-- } \\
14 \overline{4}\end{array}$ & 6 \\
\hline 4,75 & 38,62 & 1,73 & 8,13 & 463,00 & $\begin{array}{c}\text { Postos de } \\
\text { Gasolina_1_F06:94|--126 } \\
==>\text { Postos de } \\
\text { Gasolina_1_F05:62|--94 \& } \\
\text { Postos de } \\
\text { Gasolina_1_F04:50|--62 }\end{array}$ & 7 \\
\hline 4,48 & 36,40 & 1,73 & 8,13 & 463,00 & $\begin{array}{c}\text { Postos de } \\
\text { Gasolina_1_F05:62|--94 \& } \\
\text { Postos de } \\
\text { Gasolina_1_F04:50|--62 } \\
\text { ==> Postos de } \\
\text { Gasolina_1_F06:94|--126 }\end{array}$ & 8 \\
\hline 7,68 & 60,16 & 1,45 & 7,83 & 388,00 & $\begin{array}{c}\text { Postos de } \\
\text { Gasolina_1_F06:94|--126 \& } \\
\text { Postos de } \\
\text { Gasolina_1_F03:30|--50 } \\
==>\text { Postos de } \\
\text { Gasolina_1_F05:62|--94 }\end{array}$ & 10 \\
\hline 2,41 & 18,87 & 1,45 & 7,83 & 388,00 & $\begin{array}{c}\text { Postos de } \\
\text { Gasolina_1_F05:62|--94 } \\
\text { ==>Postos de } \\
\text { Gasolina_1_F06:94|--126 \& } \\
\text { Postos de } \\
\text { Gasolina_1_F03:30|--50 }\end{array}$ & 9 \\
\hline
\end{tabular}

Tabela 26: Regras de maior lift - produtos por ano

$\mathrm{Na}$ Tabela 27 observam-se regras com support elevado e valores de lift superiores ou próximos a 3. A utilização destas regras resultará em ganhos de eficiência aplicada a maior quantidade de clientes do que as enunciadas na Tabela 26. 


\begin{tabular}{|c|c|c|c|c|c|c|}
\hline $\begin{array}{c}\text { Expected } \\
\text { Confidence } \\
(\%) \\
\end{array}$ & $\begin{array}{c}\text { Confidence } \\
(\%)\end{array}$ & $\begin{array}{c}\text { Support } \\
(\%)\end{array}$ & Lift & Transações & Regra & $\begin{array}{l}\text { ID da } \\
\text { Regra }\end{array}$ \\
\hline 19,93 & 61,19 & 10,88 & 3,07 & 2912,0 & $\begin{array}{c}\text { Postos de } \\
\text { Gasolina_1_F02:18|--30 ==> } \\
\text { Postos de } \\
\text { Gasolina_1_F03:30|--50 }\end{array}$ & 9020 \\
\hline 17,78 & 54,59 & 10,88 & 3,07 & 2912,0 & $\begin{array}{l}\text { Postos de } \\
\text { Gasolina_1_F03:30|--50 ==> } \\
\text { Postos de } \\
\text { Gasolina_1_F02:18|--30 }\end{array}$ & 9019 \\
\hline 17,78 & 64,35 & 9,32 & 3,62 & 2496,0 & $\begin{array}{l}\text { Postos de } \\
\text { Gasolina_1_F01:0|--18 ==> } \\
\text { Postos de } \\
\text { Gasolina 1_F02:18|--30 }\end{array}$ & 4216 \\
\hline 14,49 & 52,45 & 9,32 & 3,62 & 2496,0 & $\begin{array}{l}\text { Postos de } \\
\text { Gasolina_1_F02:18|--30 ==> } \\
\text { Postos de } \\
\text { Gasolina_1_F01:0|--18 }\end{array}$ & 4215 \\
\hline 19,99 & 60,27 & 8,36 & 3,02 & 2238,0 & $\begin{array}{l}\text { Supermercado_1_F01:0|--13 } \\
\text { \& Farmacia_1_F02:11|--17 } \\
==>\text { Farmacia_1_F01:0|--11 }\end{array}$ & 9840 \\
\hline 13,87 & 41,82 & 8,36 & 3,02 & 2238,0 & $\begin{array}{c}\text { Farmacia_1_F01:0|--11 ==> } \\
\text { Supermercado_1_F01:0|--13 } \\
\text { \& Farmacia_1_F02:11|--17 }\end{array}$ & 9839 \\
\hline 19,93 & 63,27 & 8,05 & 3,18 & 2155,0 & $\begin{array}{l}\text { Postos de } \\
\text { Gasolina_1_F04:50|--62 ==> } \\
\text { Postos de } \\
\text { Gasolina_1_F03:30|--50 }\end{array}$ & 7723 \\
\hline 12,72 & 40,40 & 8,05 & 3,18 & 2155,0 & $\begin{array}{l}\text { Postos de } \\
\text { Gasolina_1_F03:30|--50 ==> } \\
\text { Postos de } \\
\text { Gasolina_1_F04:50|--62 }\end{array}$ & 7724 \\
\hline 23,10 & 71,77 & 7,80 & 3,11 & 2087,0 & $\begin{array}{l}\text { Farmacia_1_F03:17|--27 \& } \\
\text { Farmacia_1_F01:0|--11==> } \\
\text { Farmacia_1_F02:11|--17 }\end{array}$ & 8510 \\
\hline 10,86 & 33,76 & 7,80 & 3,11 & 2087,0 & $\begin{array}{l}\text { Farmacia_1_F02:11|--17 } \\
==>\text { Farmacia_1_F03:17|-- } \\
27 \text { \& Farmacia_1_F01:0|--11 }\end{array}$ & 8509 \\
\hline
\end{tabular}

Tabela 27: Regras de maior support-produtos por ano

Para a construção do modelo de segmentação utilizou-se uma seleção das dez mil primeiras regras de associações entre o consumo dos produtos no ano.

5.2.7 Análise de associação dos planos de pagamentos nas compras anuais

A visão anual da associação entre os planos está ilustrada na Figura 24. 


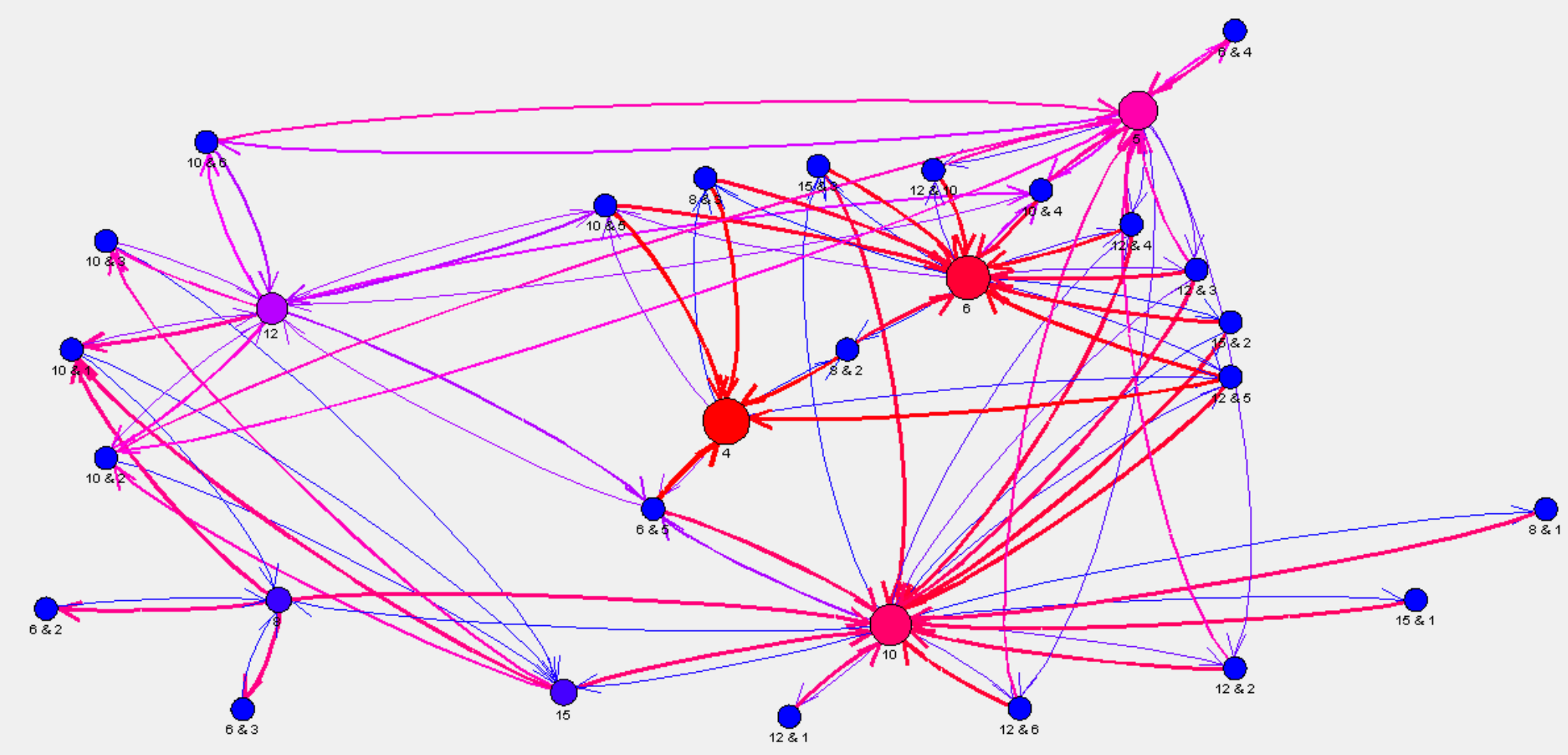

Figura 24: Grafo de associações de planos de pagamento por ano 
O Gráfico 14 apresenta a dispersão entre support e confidence das cem primeiras regras de associação do plano de pagamento na visão anual. Percebe-se um elevado número de regras com alto valor para o support e para o confidence. Contudo, o Gráfico 15, ratificado pelas Tabelas 28 e 29, demonstram regras, cujo maior lift compreende o valor de 1,98.

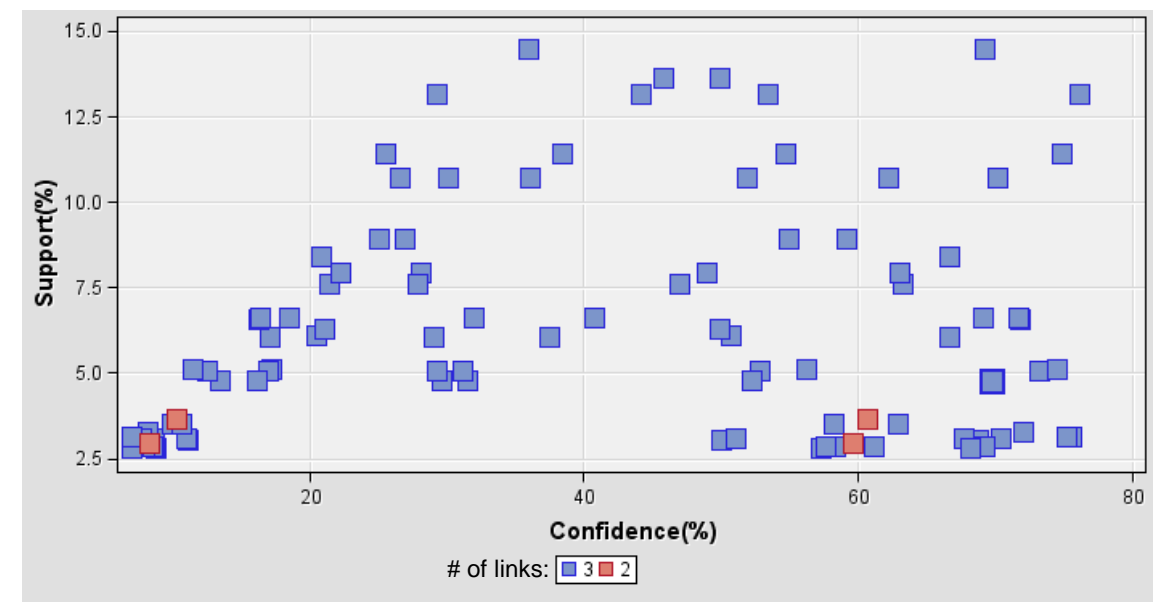

Gráfico 14: Support versus confidence para as cem primeiras regras de associação de planos de pagamentos por ano

O Gráfico 15 apresenta o confidence, expected confidence, lift e support. Na avaliação dos indicadores demonstrados, observa-se o lift praticamente constante com valores baixos, assim como na visão mensal da mesma análise e também é um indicativo que o plano de pagamento agregará pouco no modelo de segmentação de clientes, uma vez que os valores encontrados de lift não são elevados.

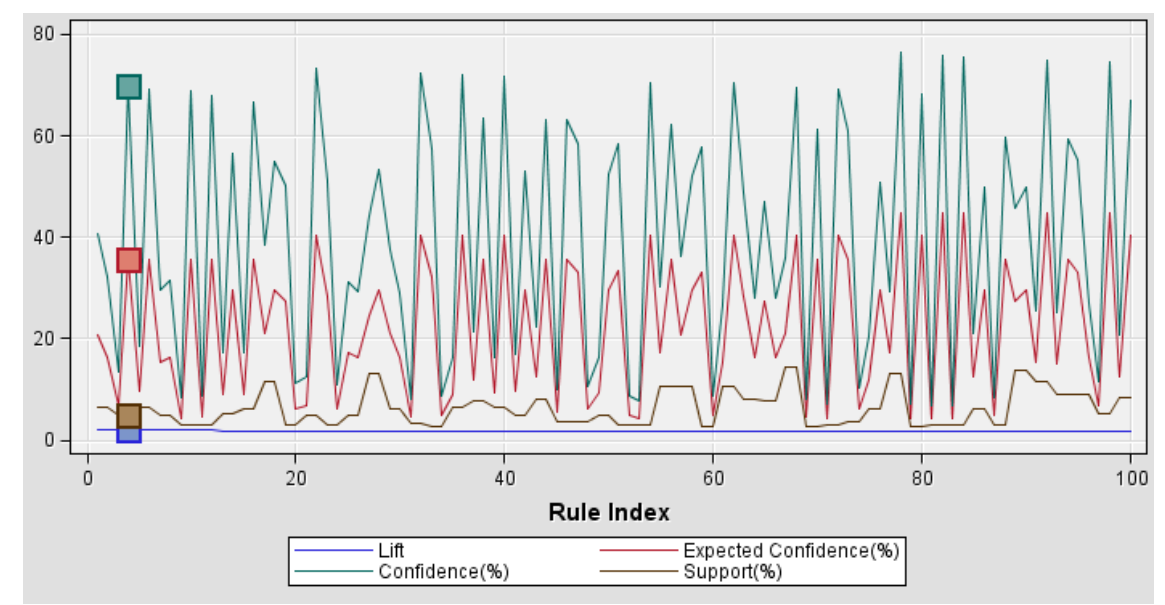

Gráfico 15: Lift, confidence, expected confidence e support de planos de pagamentos por ano 
Considerando-se os baixos ganhos que estas regras podem apresentar ao modelo de segmentação, descartou-se esta análise para a construção do modelo.

A Figura 25 apresenta a distribuição das regras encontradas na análise entre antecedentes e consequentes, bem como o confidence de cada associação por meio da escala de cores.

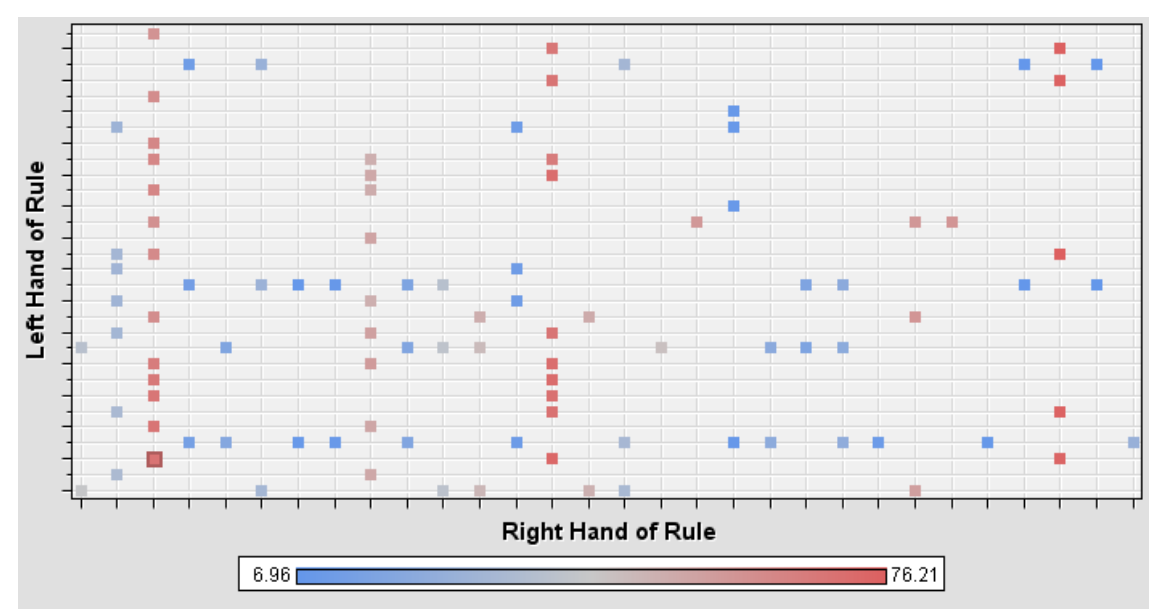

Figura 25: Matriz de regras de associação de planos de pagamentos por ano

As Tabelas 28 e 29 acusam todos os valores de lift inferiores a 2, demonstrando a não contribuição de tais regras de associação com o modelo de segmentação de clientes.

\begin{tabular}{ccccccc}
\hline $\begin{array}{c}\text { Expected } \\
\text { Confidence } \\
(\%)\end{array}$ & $\begin{array}{c}\text { Confidence } \\
(\%)\end{array}$ & $\begin{array}{c}\text { Support } \\
(\%)\end{array}$ & Lift & Transações & Regra & ID da Regra \\
\hline 20,62 & 40,73 & 6,59 & 1,98 & 1764,0 & $12==>10 \& 6$ & 1 \\
16,18 & 31,96 & 6,59 & 1,98 & 1764,0 & $10 \& 6==>12$ & 2 \\
35,52 & 69,80 & 4,80 & 1,97 & 1285,0 & $12 \& 5==>10$ & 4 \\
6,88 & 13,52 & 4,80 & 1,97 & 1285,0 & $10==>12 \& 5$ & 3 \\
35,52 & 69,12 & 6,59 & 1,95 & 1764,0 & $12 \& 6==>10$ & 6 \\
9,53 & 18,55 & 6,59 & 1,95 & 1764,0 & $10==>12 \& 6$ & 5 \\
15,25 & 29,67 & 4,80 & 1,95 & 1285,0 & $12==>10 \& 5$ & 7 \\
16,18 & 31,47 & 4,80 & 1,95 & 1285,0 & $10 \& 5==>12$ & 8 \\
35,52 & 68,76 & 3,03 & 1,94 & 810,00 & $15 \& 2==>10$ & 10 \\
4,40 & 8,52 & 3,03 & 1,94 & 810,00 & $10==>15 \& 2$ & 9 \\
\hline
\end{tabular}

Tabela 28: Regras de maior lift-planos de pagamento por ano 


\begin{tabular}{|c|c|c|c|c|c|c|}
\hline $\begin{array}{c}\text { Expected } \\
\text { Confidence } \\
(\%)\end{array}$ & $\begin{array}{c}\text { Confidence } \\
(\%)\end{array}$ & $\begin{array}{c}\text { Support } \\
(\%)\end{array}$ & Lift & Transações & Regra & ID da Regra \\
\hline 91,64 & 94,77 & 65,61 & 1,03 & 17563 & $3==>1$ & 526 \\
\hline 69,24 & 71,60 & 65,61 & 1,03 & 17563 & $1==>3$ & 527 \\
\hline 91,64 & 96,00 & 65,42 & 1,05 & 17512 & $2==>1$ & 518 \\
\hline 68,15 & 71,40 & 65,42 & 1,05 & 17512 & $1==>2$ & 519 \\
\hline 69,24 & 82,34 & 56,11 & 1,19 & 15020 & $2==>3$ & 422 \\
\hline 68,15 & 81,04 & 56,11 & 1,19 & 15020 & $3==>2$ & 421 \\
\hline 91,64 & 97,58 & 54,76 & 1,06 & 14657 & $3 \& 2==>1$ & 487 \\
\hline 69,24 & 83,70 & 54,76 & 1,21 & 14657 & $2 \& 1==>3$ & 413 \\
\hline 68,15 & 83,45 & 54,76 & 1,22 & 14657 & $3 \& 1==>2$ & 401 \\
\hline 65,61 & 80,35 & 54,76 & 1,22 & 14657 & $2==>3 \& 1$ & 400 \\
\hline
\end{tabular}

Tabela 29: Regras de maior support - planos de pagamento por ano

5.2.8 Análise de associação das cidades onde ocorreram as compras anuais

$\mathrm{Na}$ Figura 26, demonstram-se as associações entre as cidades onde ocorreram as transações no ano. Estas associações vêm reforçar a análise realizada na Seção 5.2.4 (visão mensal). 


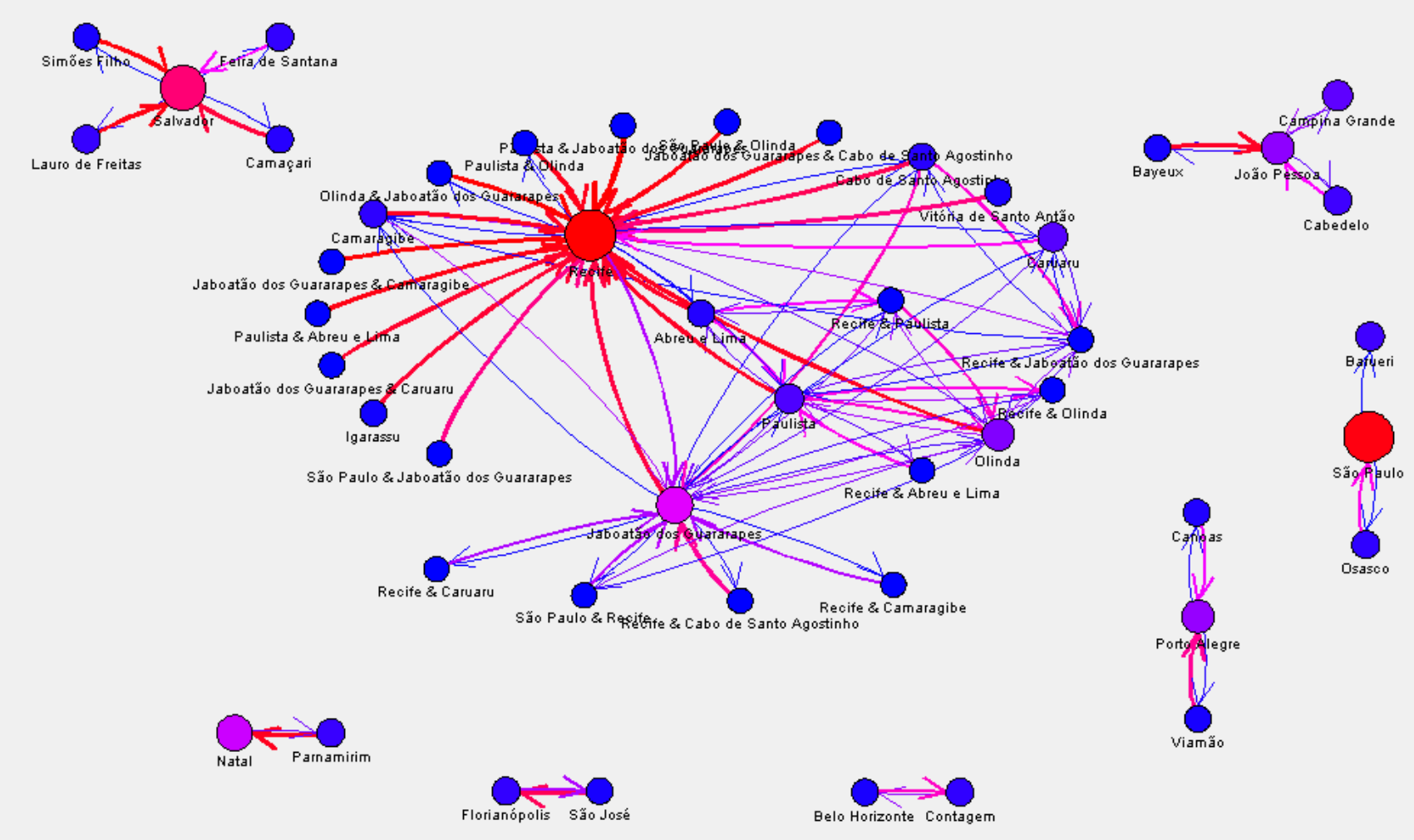

Figura 26: Grafo de associações de cidades por ano 
No Gráfico 16, é apresentado o support e o confidence das cem primeiras regras de associação de maior lift. Destaca-se neste gráfico a regra de número 24 , que tem o seguinte enunciado: Cabedelo $==>$ João Pessoa. Esta regra se destaca pelo alto valor atribuído ao lift $(12,21)$ e support $(0,97 \%)$.

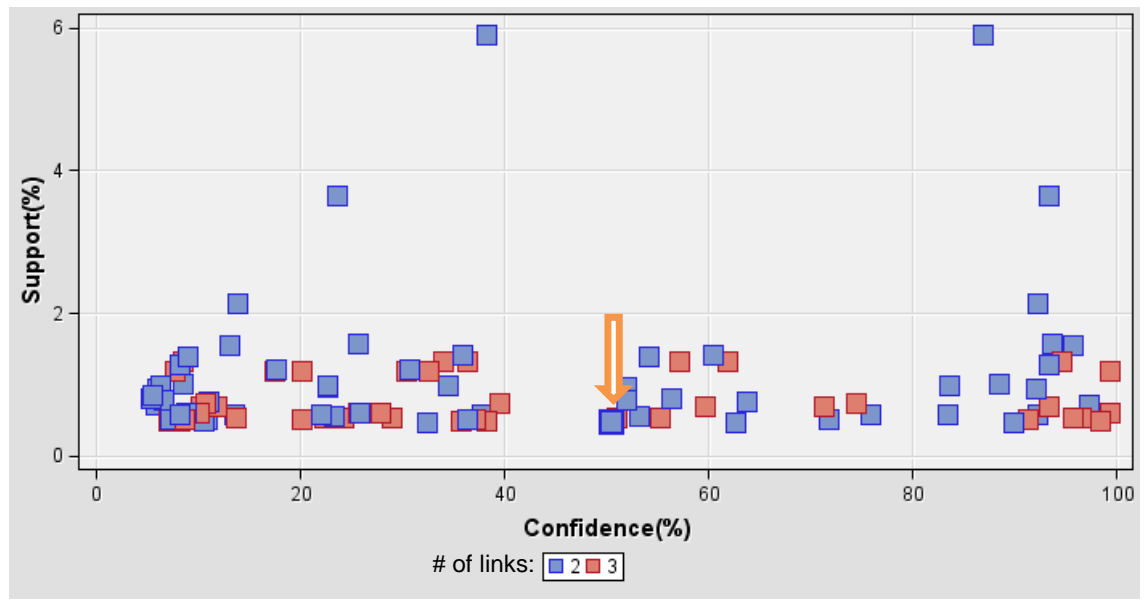

Gráfico 16: Support versus confidence para as cem primeiras regras de associação de cidades por ano

A avaliação dos indicadores demonstrados no Gráfico 17 indica que a análise da cidade onde a compra foi realizada resulta em ganho de eficiência no modelo de segmentação de clientes, uma vez que alguns valores encontrados de lift são elevados.

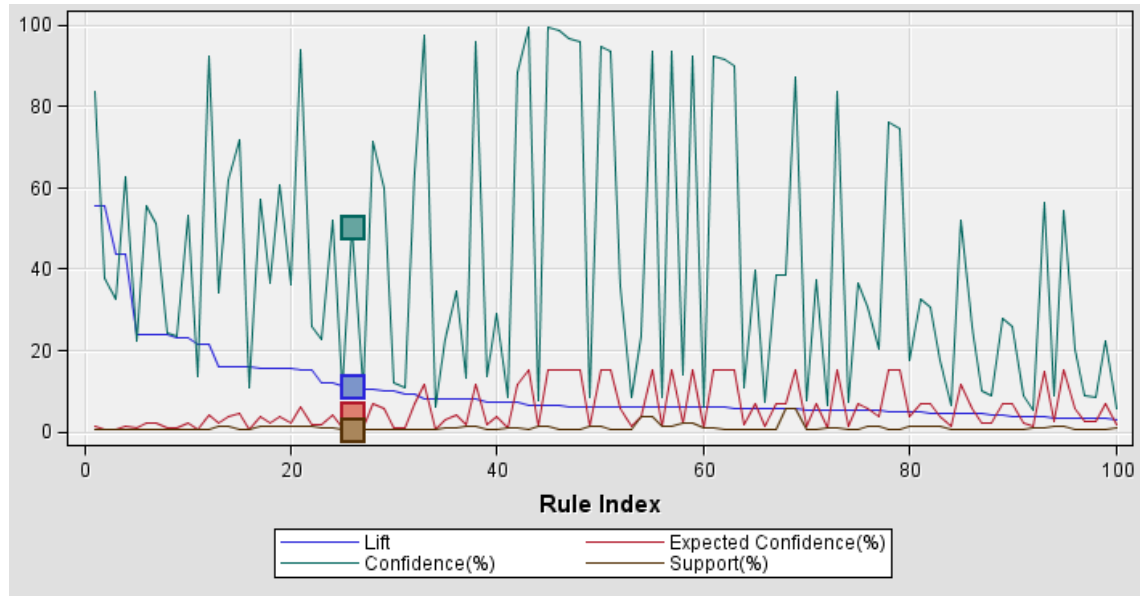

Gráfico 17: Lift, confidence, expected confidence e support de cidades por ano 
A Figura 27 apresenta a distribuição das regras encontradas na análise entre antecedentes e consequentes, bem como o confidence de cada associação por meio da escala de cores.

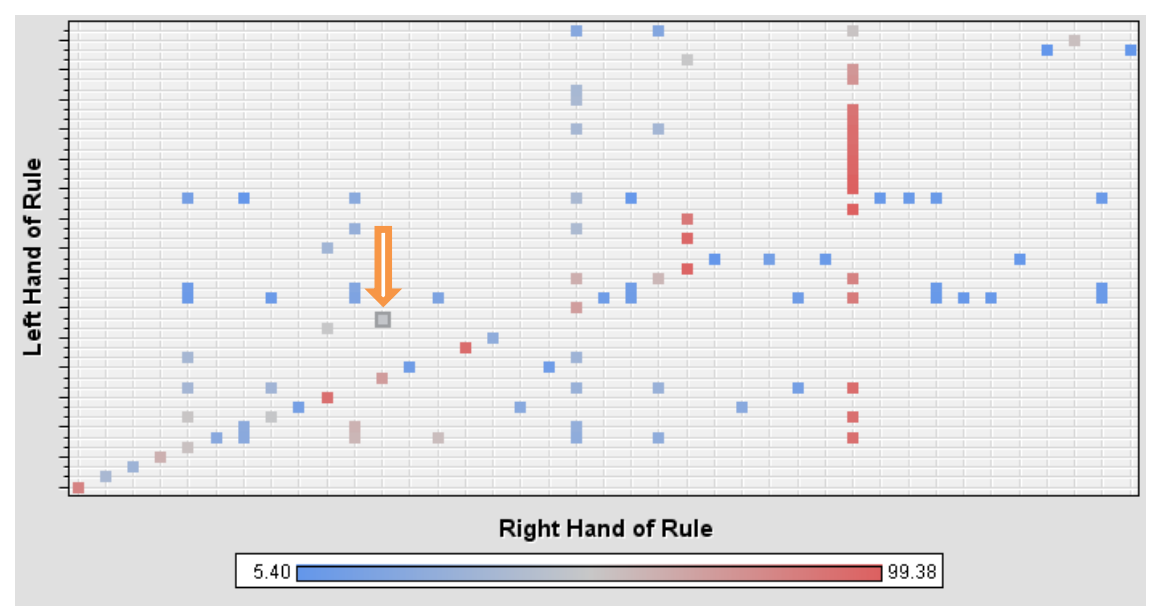

Figura 27: Matriz de regras de associação de cidades por ano

Embora algumas associações tenham o lift muito elevado (acima de 20), o número de ocorrências de tais associações é baixo. Estas informações simplificam o modelo de segmentação, pois agrupam os clientes segundo as cidades onde compram, mesmo que seja necessário o deslocamento no espaço, em relação à cidade de origem do cliente.

Verifica-se na Tabela 30, as regras com altos valores para o lift, contudo os valores de support são inferiores a 1\%, o que vem a ser um indicativo de que estas regras, embora se apliquem a poucos casos, contribuirão com a eficiência do modelo de segmentação. 


\begin{tabular}{|c|c|c|c|c|c|c|}
\hline $\begin{array}{c}\text { Expected } \\
\text { Confidence } \\
(\%)\end{array}$ & $\begin{array}{c}\text { Confidence } \\
(\%)\end{array}$ & $\begin{array}{c}\text { Support } \\
(\%)\end{array}$ & Lift & Transações & Regra & ID da Regra \\
\hline 1,51 & 83,61 & 0,57 & 55,39 & 153,00 & $\begin{array}{l}\text { São José }==> \\
\text { Florianópolis }\end{array}$ & 1 \\
\hline 0,68 & 37,87 & 0,57 & 55,39 & 153,00 & $\begin{array}{l}\text { Florianópolis ==> } \\
\text { São José }\end{array}$ & 2 \\
\hline 0,74 & 32,55 & 0,47 & 43,79 & 125,00 & $\begin{array}{c}\text { Contagem }==>\text { Belo } \\
\text { Horizonte }\end{array}$ & 3 \\
\hline 1,43 & 62,81 & 0,47 & 43,79 & 125,00 & $\begin{array}{c}\text { Belo Horizonte }==> \\
\text { Contagem }\end{array}$ & 4 \\
\hline 2,32 & 55,38 & 0,52 & 23,91 & 139,00 & $\begin{array}{c}\text { Recife \& Abreu e } \\
\text { Lima }==>\text { Paulista }\end{array}$ & 6 \\
\hline 0,94 & 22,42 & 0,52 & 23,91 & 139,00 & $\begin{array}{c}\text { Paulista }==>\text { Recife } \\
\text { \& Abreu e Lima }\end{array}$ & 5 \\
\hline 1,02 & 24,26 & 0,52 & 23,87 & 139,00 & $\begin{array}{l}\text { Recife \& Paulista } \\
==>\text { Abreu e Lima }\end{array}$ & 8 \\
\hline 2,14 & 51,10 & 0,52 & 23,87 & 139,00 & $\begin{array}{l}\text { Abreu e Lima ==> } \\
\text { Recife \& Paulista }\end{array}$ & 7 \\
\hline 1,02 & 23,39 & 0,54 & 23,01 & 145,00 & $\begin{array}{c}\text { Paulista }==>\text { Abreu } \\
\text { e Lima }\end{array}$ & 9 \\
\hline 2,32 & 53,31 & 0,54 & 23,01 & 145,00 & $\begin{array}{c}\text { Abreu e Lima }==> \\
\text { Paulista }\end{array}$ & 10 \\
\hline
\end{tabular}

Tabela 30: Regras de maior lift - cidades por ano

$\mathrm{Na}$ Tabela 31 observam-se regras com support elevado e valores de lift elevados. A utilização destas regras resultará em ganhos de eficiência aplicada a maior quantidade de clientes do que as enunciadas na Tabela 30.

\begin{tabular}{|c|c|c|c|c|c|c|}
\hline $\begin{array}{c}\text { Expected } \\
\text { Confidence } \\
(\%)\end{array}$ & $\begin{array}{c}\text { Confidence } \\
(\%)\end{array}$ & $\begin{array}{c}\text { Support } \\
(\%)\end{array}$ & Lift & Transações & Regra & ID da Regra \\
\hline 6,77 & 38,40 & 5,89 & 5,67 & 1577,0 & $\begin{array}{c}\text { Recife }==>\text { Jaboatão } \\
\text { dos Guararapes }\end{array}$ & 68 \\
\hline 15,34 & 87,03 & 5,89 & 5,67 & 1577,0 & $\begin{array}{c}\text { Jaboatão dos } \\
\text { Guararapes ==> } \\
\text { Recife }\end{array}$ & 69 \\
\hline 3,89 & 23,69 & 3,64 & 6,09 & 973,00 & Recife $==>$ Olinda & 54 \\
\hline 15,34 & 93,47 & 3,64 & 6,09 & 973,00 & Olinda $==>$ Recife & 55 \\
\hline 2,32 & 13,95 & 2,14 & 6,02 & 573,00 & Recife $==>$ Paulista & 58 \\
\hline 15,34 & 92,42 & 2,14 & 6,02 & 573,00 & Paulista $==>$ Recife & 59 \\
\hline 15,34 & 12,44 & 1,85 & 0,81 & 495,00 & $\begin{array}{c}\text { São Paulo ==> } \\
\text { Recife }\end{array}$ & 121 \\
\hline 14,87 & 12,05 & 1,85 & 0,81 & 495,00 & $\begin{array}{c}\text { Recife }==>\text { São } \\
\text { Paulo }\end{array}$ & 122 \\
\hline 6,12 & 93,76 & 1,57 & 15,33 & 421,00 & $\begin{array}{c}\text { Parnamirim } \\
\text { Natal }\end{array}==>$ & 21 \\
\hline 1,68 & 25,72 & 1,57 & 15,33 & 421,00 & $\begin{array}{c}\text { Natal }==> \\
\text { Parnamirim }\end{array}$ & 22 \\
\hline
\end{tabular}

Tabela 31: Regras de maior support - cidades por ano

\subsection{Aplicação da análise de cesto de compras a base de modelagem}

A aplicação da ACC conta com a base de transações utilizada na aplicação da $A A$, além de uma base de taxonomia, na qual os produtos são agrupados em 
suas famílias. Este agrupamento resulta no levantamento de regras de associação, em qualquer nível da hierarquia definida para cada um dos produtos.

Na Figura 28, observa-se um exemplo da classificação de cada produto em sua família.

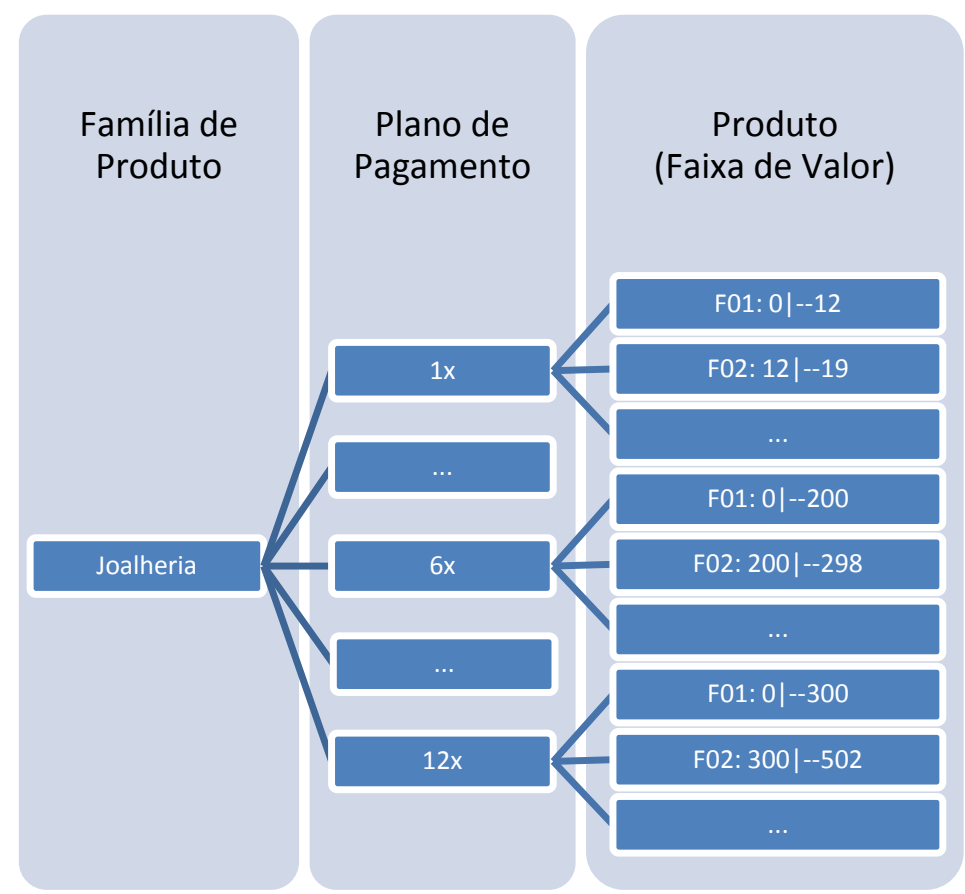

Figura 28: Exemplo da taxonomia dos produtos

Além de utilizar a classificação das famílias de produtos, a nova análise introduz uma medida às associações. Esta medida, denominada support lift, indica quais são as regras mais interessantes, baseada no desvio do support esperado (support do produto em seu nível hierárquico imediatamente superior).

O algoritmo aplicado de ACC foi aplicado sobre base de compras de produtos agrupadas em faturas mensais do cartão de crédito de cada cliente. A base de faturas analisada possui 26.767 clientes, 171.667 faturas e 787.986 transações ou compras de produtos.

A aplicação do algoritmo resultou em uma seleção de 6.537 regras de associação. 
O Gráfico 18 mostra o conjunto de regras levantadas a partir da análise, ordenadas pelo lift, no mesmo conceito já descrito na Seção de AA.

Percebe-se que a regra de maior lift identificada, o apresenta superior a 40. $\mathrm{O}$ lift se mantém bastante elevado e superior a 2 até a regra de número 2.582. Estas serão as regras consideradas no modelo de segmentação.

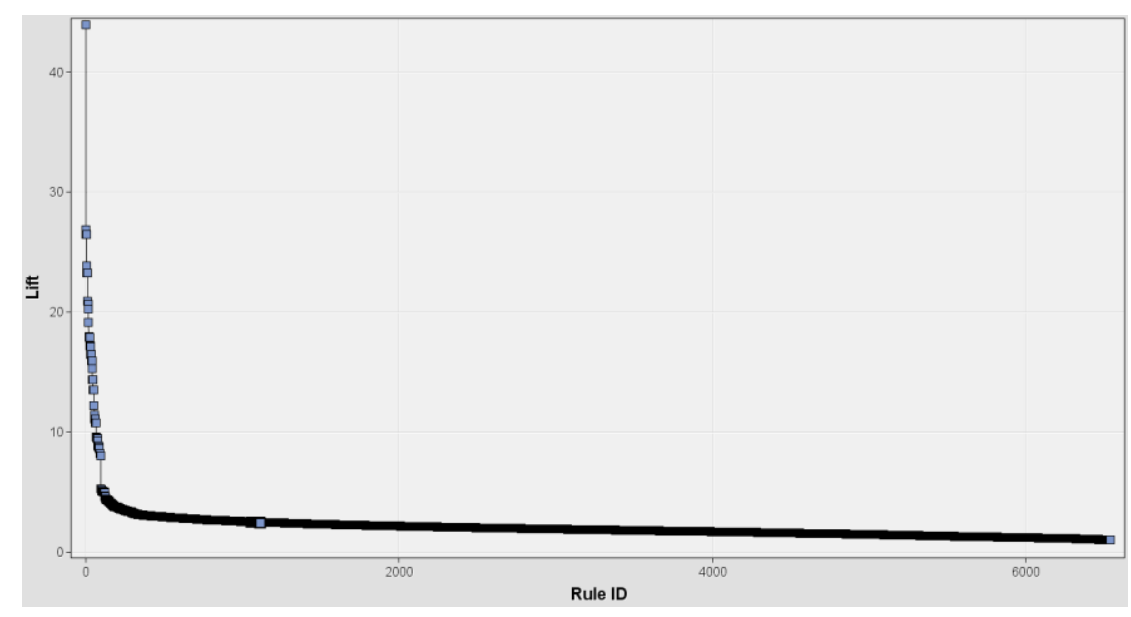

Gráfico 18: Análise de cesto de compras - 6.537 regras ordenadas por lift

O Gráfico 19 ilustra a dispersão das regras, em relação à representatividade das transações (support) e a força da associação (confidence).

Observa-se uma grande quantidade de pontos representando regras com elevados valores de confidence elevado (acima de 40), além de regras com valores de support acima de $5 \%$, ou seja, regras que podem incrementar a eficiência da comunicação atingindo até $5 \%$ dos clientes, o que é considerado elevado. 


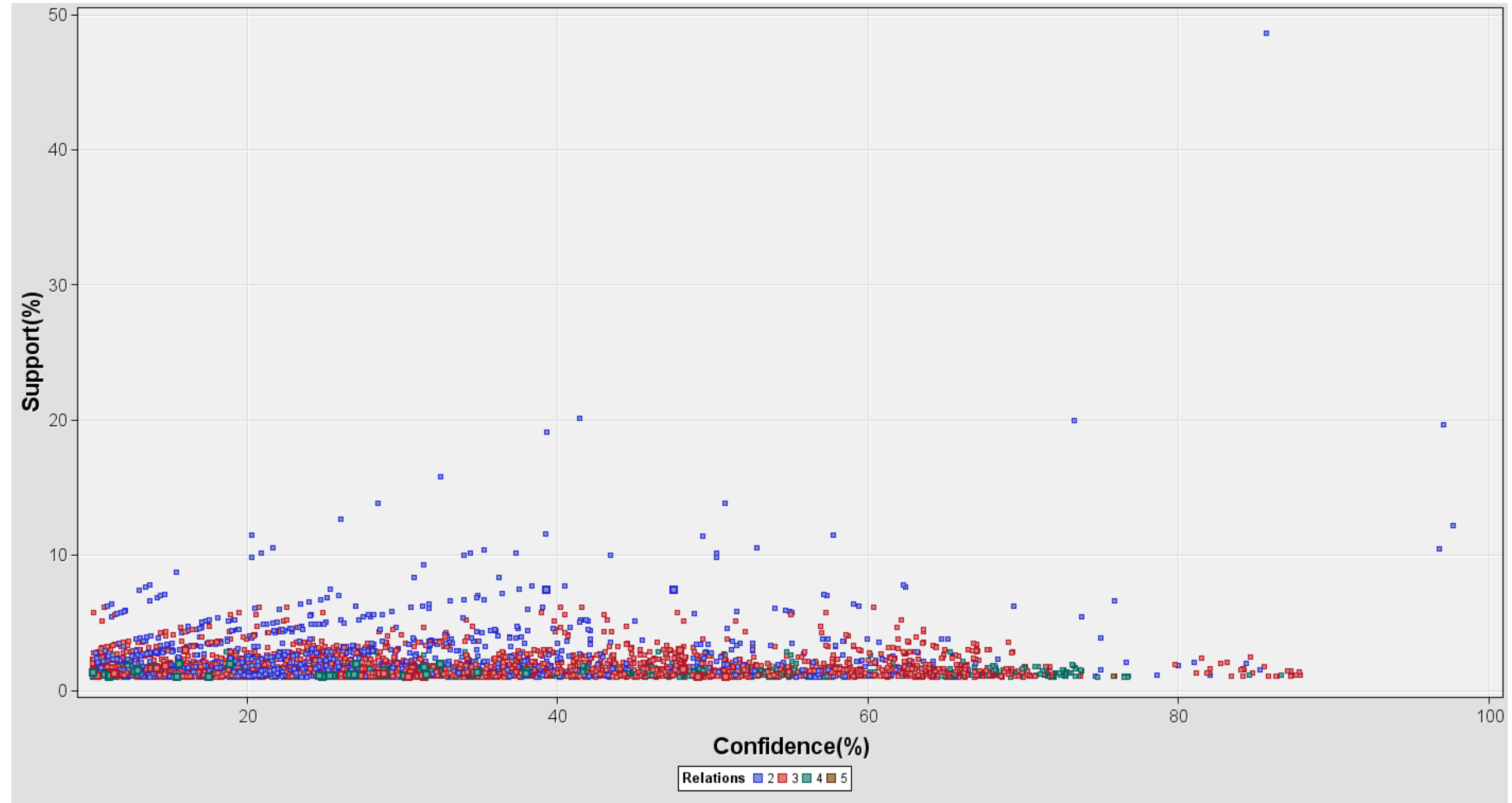

Gráfico 19: Dispersão das regras em relação ao support e confidence 
A Tabela 32 mostra as 20 regras de maior support lift entre as 6.537 regras encontradas na análise. É importante observar que, ao ordenar as regras por este critério, agrupam-se as regras referentes aos mesmos produtos; ou seja, aproximam-se as combinações possíveis decorrentes da ordem de consumo dos produtos. No entanto, nota-se diferenças entre os lifts das regras sobre os mesmos produtos, o que ratifica que a ordem de consumo dos produtos deve induzir ao modelo de segmentação.

\begin{tabular}{|c|c|c|c|c|c|}
\hline Quantidade & $\begin{array}{l}\text { Support } \\
(\%)\end{array}$ & $\begin{array}{l}\text { Support } \\
\text { Lift (\%) }\end{array}$ & Lift & Regra & $\begin{array}{l}\text { ID da } \\
\text { Regra }\end{array}$ \\
\hline 83.500 & 48,64 & 6,70 & 1,76 & Supermercado $==>$ Supermercado $\_1$ & 3.706 \\
\hline 19.587 & 11,40 & 1,95 & 4,32 & Lojas em Geral ==> Lojas em Geral_1 & 139 \\
\hline 2.000 & 1,165 & 1,95 & 3,74 & $\begin{array}{l}\text { Farmacia_1_F01:0|--11 \& Supermercado_1_F02:13|-- } \\
28==>\text { Supermercado_1_F01:0|--13 }\end{array}$ & 200 \\
\hline 2.000 & 1,165 & 1,95 & 3,03 & $\begin{array}{l}\text { Farmacia_1_F01:0|--11 \& Supermercado_1_F01:0|-- } \\
13==>\text { Supermercado_1_F02:13|--28 }\end{array}$ & 396 \\
\hline 2.000 & 1,165 & 1,95 & 2,65 & $\begin{array}{l}\text { Farmacia_1_F01:0|--11 = => Supermercado_1_F01:0|-- } \\
13 \text { \& Supermercado_1_F02:13|--28 }\end{array}$ & 823 \\
\hline 2.000 & 1,165 & 1,95 & 2,65 & $\begin{array}{l}\text { Supermercado_1_F01:0|--13 \& } \\
\text { Supermercado_1_F02:13|--28 ==> } \\
\text { Farmacia_1_F01:0|--11 }\end{array}$ & 824 \\
\hline 34.276 & 19,96 & 1,93 & 3,67 & Farmácia $==>$ Farmacia 1 & 214 \\
\hline 34.608 & 20,15 & 1,90 & 2,05 & Supermercado 1 ==> Supermercado 1 F03:28|--61 & 2.380 \\
\hline 32.804 & 19,10 & 1,75 & 2,05 & Supermercado 1 ==> Supermercado 1 F02:13|--28 & 2.379 \\
\hline 2.394 & 1,39 & 1,60 & 4,35 & $\begin{array}{l}\text { Supermercado_1_F01:0|--13 \& } \\
\text { Supermercado_1_F03:28|--61 ==> Farmacia_1 \& } \\
\text { Supermercado_1_F02:13|--28 }\end{array}$ & 133 \\
\hline 2.394 & 1,39 & 1,60 & 4,35 & $\begin{array}{l}\text { Farmacia_1 \& Supermercado_1_F02:13|--28 ==> } \\
\text { Supermercado_1_F01:0|--13 \& } \\
\text { Supermercado_1_F03:28|--61 }\end{array}$ & 134 \\
\hline 2.394 & 1,39 & 1,60 & 3,85 & $\begin{array}{l}\text { Farmacia_1 \& Supermercado_1_F01:0|--13==> } \\
\text { Supermercado_1_F02:13|--28 \& } \\
\text { Supermercado_1_F03:28|--61 }\end{array}$ & 181 \\
\hline 2.394 & 1,39 & 1,60 & 3,85 & $\begin{array}{l}\text { Supermercado_1_F02:13|--28 \& } \\
\text { Supermercado_1_F03:28|--61 ==> Farmacia_1 \& } \\
\text { Supermercado_1_F01:0|--13 }\end{array}$ & 182 \\
\hline 2.394 & 1,39 & 1,60 & 3,78 & $\begin{array}{l}\text { Farmacia_1 \& Supermercado_1_F03:28|--61 ==> } \\
\text { Supermercado_1_F01:0|--13 \& } \\
\text { Supermercado_1_F02:13|--28 }\end{array}$ & 185 \\
\hline 2.394 & 1,39 & 1,60 & 3,78 & $\begin{array}{l}\text { Supermercado_1_F01:0|--13 \& } \\
\text { Supermercado_1_F02:13|--28 ==> Farmacia_1 \& } \\
\text { Supermercado_1_F03:28|--61 }\end{array}$ & 186 \\
\hline 2.394 & 1,39 & 1,60 & 3,67 & $\begin{array}{l}\text { Farmacia_1 \& Supermercado_1_F02:13|--28 \& } \\
\text { Supermercado_1_F03:28|--61_==> } \\
\text { Supermercado_1_F01:0|--13 }\end{array}$ & 209 \\
\hline 2.394 & 1,39 & 1,60 & 3,53 & $\begin{array}{l}\text { Farmacia_1 \& Supermercado_1_F01:0|--13 \& } \\
\text { Supermercado_1_F03:28|--61 ==> } \\
\text { Supermercado_1_F02:13|--28 }\end{array}$ & 241 \\
\hline 2.394 & 1,39 & 1,60 & 2,70 & $\begin{array}{l}\text { Farmacia_1 \& Supermercado_1_F01:0|--13 \& } \\
\text { Supermercado_1_F02:13|--28==> } \\
\text { Supermercado_1_F03:28|--61 }\end{array}$ & 767 \\
\hline 2.394 & 1,39 & 1,60 & 1,82 & $\begin{array}{l}\text { Supermercado_1_F01:0|--13 \& } \\
\text { Supermercado_1_F02:13|--28 \& } \\
\text { Supermercado_1_F03:28|--61 ==> Farmacia_1 }\end{array}$ & 3.390 \\
\hline 1.727 & 1,00 & 1,53 & 5,02 & $\begin{array}{l}\text { Supermercado_1_F01:0|--13 \& } \\
\text { Supermercado_1_F03:28|--61 ==> } \\
\text { Supermercado_1_F02:13|--28 \& Supermercado_3 }\end{array}$ & 116 \\
\hline
\end{tabular}

Tabela 32: 20 primeiras regras ordenadas pelo support lift 
Analisando o conjunto total de 6.537 regras encontradas a partir da ACC, afere-se 759 antecedentes e 280 consequentes. Tanto os antecedentes, quanto os consequentes, podem ser combinações de diferentes níveis da taxonomia.

\subsection{Método de definição das regras para a segmentação de clientes}

Para a modelagem da segmentação dos clientes de cartão de credito a partir das bases transacionais, utilizou-se: as regras extraídas da ACC; as associações simples entre famílias de produtos, produtos, planos de pagamento e cidades de consumo, resultantes da AA.

Parte-se das transações consequentes que coincidem com a oferta que se deseja comunicar aos clientes, para então se ter a seleção das compras antecedentes mais frequentes, indicativas da formação um conjunto de regras de segmentação de clientes de acordo com o uso do cartão de crédito. O critério fundamental para a utilização das regras na segmentação é que o lift calculado para a regra seja superior a 2 .

Realizou-se, até o final desta seção, passo a passo, a seleção das regras para a segmentação do público para o recebimento de uma oferta exemplo.

A oferta utilizada como exemplo é de uma rede de lojas de joias, com abrangência nacional, de produtos com valores e prazos de pagamentos variados, a ser feita no mês de Julho. Para essa finalidade, utilizou-se os dados dos meses de janeiro a junho para a segmentação do público.

É sabido, pela da análise da Tabela 13, que as compras em joalherias representam $1,25 \%$ das transações ocorridas no ano. Comunicando todos os clientes (38.357) sobre a oferta, o potencial esperado de transações de um mês é de 818 compras, que indica um resultado de uma transação a cada 47 comunicações; ou seja, uma eficiência esperada de $2,1 \%$. 
Sabe-se também que na base de modelagem, no mês de Julho, foram realizadas 752 compras em Joalherias, por 677 clientes diferentes. O desafio é escolher a melhor regra para encontrar uma grande quantidade destes clientes que compraram em joalherias em Julho, com dados registrados até o mês anterior.

Primeiramente analisou-se as relações de transações antecedentes que resultaram em consequentes da família de produtos Joalheria na ACC (Tabela 33).

\begin{tabular}{|c|c|c|c|c|c|}
\hline $\begin{array}{l}\text { Consequente } \\
\text { Antecedente }\end{array}$ & ID da regra & Lift. & Support Lift & Confidence (\%) & Support (\%) \\
\hline \multicolumn{6}{|l|}{ Joalherias } \\
\hline Lojas em Geral \& Vestuário & 1.287 & 2,41 & 0,00 & 11,93 & 1,20 \\
\hline Farmácia \& Vestuário & 1.570 & 2,30 & 0,00 & 11,34 & 1,15 \\
\hline Vestuario_2 & 2.269 & 2,09 & 0,22 & 10,32 & 1,08 \\
\hline Supermercado_1 \& & 2.311 & 2,08 & 0,05 & 10,27 & 1,34 \\
\hline \multicolumn{5}{|l|}{ Vestuário } & 1,49 \\
\hline \multicolumn{6}{|l|}{ Joalherias_1 } \\
\hline Joalherias & 17 & 20,24 & $-0,62$ & 37,75 & 1,87 \\
\hline
\end{tabular}

Tabela 33: Regras da ACC para o consequente joalheria e joalheria_1

Em segundo lugar, realizou-se a mesma verificação com as regras $A A$, para todas as regras encontradas nas análises apresentadas nas Seções 5.2.1 até 5.2.8.

Da análise de associação das famílias de produtos nas compras mensais, extraíram-se as regras listadas na Tabela 34.

\begin{tabular}{|c|c|c|c|c|}
\hline $\begin{array}{l}\text { Consequente } \\
\text { Antecedente }\end{array}$ & ID da regra & Lift & $\begin{array}{c}\text { Confidence } \\
\text { (\%) }\end{array}$ & $\begin{array}{l}\text { Support } \\
\text { (\%) }\end{array}$ \\
\hline \multicolumn{5}{|l|}{ Joalherias } \\
\hline Vestuário \& Diversão & 5 & 2,92 & 14,45 & 0,75 \\
\hline Lojas em Geral \& Diversão & 22 & 2,66 & 13,13 & 0,59 \\
\hline Vestuário \& Lojas em Geral & 83 & 2,41 & 11,93 & 1,2 \\
\hline Farmácia \& Diversão & 107 & 2,33 & 11,51 & 0,6 \\
\hline Vestuário \& Farmácia & 121 & 2,3 & 11,34 & 1,15 \\
\hline Lojas em Geral \& Farmácia & 173 & 2,17 & 10,7 & 0,9 \\
\hline Vestuário \& Postos de Gasolina & 207 & 2,12 & 10,45 & 0,61 \\
\hline Supermercado \& Diversão & 251 & 2,05 & 10,11 & 0,79 \\
\hline Vestuário \& Supermercado & 263 & 2,04 & 10,06 & 1,49 \\
\hline Perfumaria & 275 & 2,02 & 9,98 & 0,69 \\
\hline
\end{tabular}


Para acrescentar alguma observação que pode ter sido prejudicada pelo período mensal de análise, verificou-se a regra de associação levando-se em consideração transações do ano, com lift também superior a 2 demonstradas na Tabela 35, que contribuirão com os critérios de segmentação dos clientes para a recepção da oferta da Joalheria do exemplo.

\begin{tabular}{|c|c|c|c|c|}
\hline $\begin{array}{l}\text { Consequente } \\
\text { Antecedente }\end{array}$ & ID da regra & Lift & $\begin{array}{l}\text { Confidence } \\
(\%)\end{array}$ & $\begin{array}{l}\text { Support } \\
\text { (\%) }\end{array}$ \\
\hline \multicolumn{5}{|l|}{ Joalherias } \\
\hline Perfumaria \& Móveis & 10 & 2,45 & 52,8 & 3,49 \\
\hline Perfumaria \& Geral & 22 & 2,38 & 51,24 & 4,39 \\
\hline Móveis \& Livraria & 24 & 2,37 & 51,24 & 2,71 \\
\hline Perfumaria \& Livraria & 32 & 2,35 & 50,67 & 4,12 \\
\hline Serviços \& Perfumaria & 34 & 2,33 & 50,17 & 5,51 \\
\hline Serviços \& Livraria & 50 & 2,29 & 49,5 & 4,45 \\
\hline Serviços \& Móveis & 62 & 2,27 & 49,05 & 3,76 \\
\hline Perfumaria \& Diversão & 64 & 2,27 & 49,02 & 5,86 \\
\hline Livraria \& Geral & 84 & 2,24 & 48,33 & 3,4 \\
\hline Móveis \& Diversão & 98 & 2,23 & 48,05 & 3,68 \\
\hline Serviços \& Geral & 104 & 2,22 & 47,94 & 4,7 \\
\hline Veículos \& Perfumaria & 112 & 2,21 & 47,65 & 3,59 \\
\hline Móveis \& Geral & 114 & 2,21 & 47,64 & 3,01 \\
\hline Perfumaria \& Alimentos & 140 & 2,19 & 47,27 & 4,46 \\
\hline $\begin{array}{l}\text { Telecomunicação / Eletrônicosco \& } \\
\text { Perfumaria }\end{array}$ & 164 & 2,18 & 46,95 & 5,92 \\
\hline Geral \& Diversão & 194 & 2,16 & 46,54 & 4,74 \\
\hline Livraria \& Diversão & 192 & 2,16 & 46,54 & 4,74 \\
\hline Livraria \& Alimentos & 220 & 2,15 & 46,31 & 3,68 \\
\hline Serviços \& Diversão & 282 & 2,12 & 45,7 & 6,29 \\
\hline Perfumaria \& Construção & 280 & 2,12 & 45,73 & 5,18 \\
\hline $\begin{array}{l}\text { Telecomunicação / Eletrônicosco \& } \\
\text { Livraria }\end{array}$ & 296 & 2,11 & 45,59 & 4,86 \\
\hline Móveis \& Alimentos & 344 & 2,09 & 45,01 & 2,93 \\
\hline Postos de Gasolina \& Perfumaria & 354 & 2,08 & 44,92 & 4,92 \\
\hline Perfumaria \& Lojas em Geral & 376 & 2,07 & 44,74 & 8,53 \\
\hline Perfumaria \& Farmácia & 380 & 2,07 & 44,71 & 8,22 \\
\hline Geral \& Alimentos & 448 & 2,05 & 44,15 & 3,99 \\
\hline $\begin{array}{l}\text { Telecomunicação / Eletrônicosco \& } \\
\text { Serviços }\end{array}$ & 436 & 2,05 & 44,22 & 6,5 \\
\hline Serviços \& Alimentos & 490 & 2,03 & 43,72 & 4,99 \\
\hline $\begin{array}{l}\text { Telecomunicação / Eletrônicosco \& } \\
\text { Geral }\end{array}$ & 494 & 2,03 & 43,71 & 5,03 \\
\hline Livraria \& Construção & 508 & 2,02 & 43,57 & 4,23 \\
\hline Livraria \& Farmácia & 510 & 2,02 & 43,56 & 6,51 \\
\hline $\begin{array}{l}\text { Telecomunicação / Eletrônicosco \& } \\
\text { Móveis }\end{array}$ & 542 & 2 & 43,22 & 4,21 \\
\hline
\end{tabular}


O terceiro, o quarto e o quinto passos a seguir são equivalentes ao segundo para a associação dos produtos, prazos e cidades onde as transações deverão ocorrer.

Sendo assim, baseado nas Seções 5.2.2 e 5.2.6, concluiu-se que não existem regras de associação que cumpram os requisitos mínimos nas transações mensais. Entretanto, nas transações anuais, foi encontrada uma regra de associação, conforme está descrita na Tabela 36.

\begin{tabular}{|c|c|c|c|c|}
\hline $\begin{array}{l}\text { Consequente } \\
\text { Antecedente }\end{array}$ & ID da regra & Lift & $\begin{array}{c}\text { Confidence } \\
\text { (\%) }\end{array}$ & $\begin{array}{c}\text { Support } \\
\text { (\%) }\end{array}$ \\
\hline \multicolumn{5}{|l|}{ Joalherias_1_F02:12|--19 } \\
\hline Vestuario_1_F02:15|--24 & 8747 & 3,09 & 8,54 & 1,45 \\
\hline
\end{tabular}

Como a oferta não possui um prazo específico de pagamento e é de abrangência nacional, as análises do quarto e quinto passos não representam enriquecimento para a regra de segmentação do público, na condição de oferta deste exemplo.

Finalmente, a regra de segmentação de clientes da oferta de uma rede de lojas de joias com abrangência nacional, de produtos com valores e prazos de pagamentos variados, está descrita na Expressão lógica 8. 
[(Lojas em Geral E Vestuário)

OU (Farmácia E Vestuário

OU (Vestuario 2

OU (Supermercado $1 \mathrm{E}$ Vestuário

OU (Supermercado E Vestuário

OU (Joalherias)]

OU clientes que tenham comprado no mês

[(Vestuário E Diversão

OU (Lojas em Geral E Diversão)

OU (Vestuário E Lojas em Geral)

OU (Farmácia E Diversão)

OU (Vestuário E Farmácia)

OU (Lojas em Geral E Farmácia)

OU (Vestuário E Postos de Gasolina)

OU (Supermercado E Diversão)

OU (Vestuário E Supermercado)

OU (Perfumaria)]

OU clientes que tenham comprado no ano

[(Perfumaria E Móveis)

OU (Perfumaria E Geral)

OU (Móveis E Livraria)

OU (Perfumaria E Livraria)

OU (Serviços E Perfumaria)

OU (Serviços E Livraria)

OU (Serviços E Móveis)

OU (Perfumaria E Diversão)

OU (Livraria E Geral)

OU (Móveis E Diversão)

OU (Serviços E Geral)

OU (Veículos E Perfumaria)

OU (Móveis E Geral)

OU (Perfumaria E Alimentos)

OU (Telecomunicação / Eletrônicosco E Perfumaria)

OU (Geral E Diversão)

OU (Livraria E Diversão)

OU (Livraria E Alimentos)

OU (Serviços E Diversão)

OU (Perfumaria E Construção)

OU (Telecomunicação / Eletrônicosco E Livraria)

OU (Móveis E Alimentos)

OU (Postos de Gasolina E Perfumaria)

OU (Perfumaria E Lojas em Geral)

OU (Perfumaria E Farmácia)

OU (Geral E Alimentos)

OU (Telecomunicação / Eletrônicosco E Serviços)

OU (Serviços E Alimentos)

OU (Telecomunicação / Eletrônicosco E Geral)

OU (Livraria E Construção)

OU (Livraria E Farmácia)

OU (Telecomunicação / Eletrônicosco E Móveis)]

OU clientes que tenham comprado no ano

[(Vestuario_1_F02:15|--24)]

Para saber quanto este segmento de clientes incrementa a eficiência inicial de uma ação de comunicação para toda a base, calculada anteriormente em 2,1\%, fazse necessária a seleção dos clientes, de acordo com a Expressão 8.

O grupo de clientes selecionados, conforme a regra indicada na expressão 8 , contém 11.394 clientes e ao conferir quantos deles realizaram compras em Joalherias no mês de Julho, observou-se que 506 compraram, ou seja, mediante a abordagem de $29,7 \%$ do público, sensibilizaria-se $74,7 \%$ dos clientes que realizariam as compras em Joalherias, resultando em uma eficiência de 4,4\%, e um lift de 2,09. 
A evidência desta demonstração simples ainda não coloca à prova o modelo de segmentação dos clientes de cartão de crédito por perfil de consumo, pois todas estas compras e clientes encontrados foram utilizados para a geração das regras de associação. No entanto, nas próximas seções, apresenta-se a criação do modelo de segmentação dos clientes cujos testes realizar-se-ão sobre uma amostra de validação que não influenciou na geração das regras.

\subsection{Segmentação do cliente de cartão de crédito}

De posse das análises relatadas na Seção 5.2.2, conclui-se que existe concentração no consumo de poucos produtos que por vezes são associados ao consumo de uma maior diversidade de produtos.

A concentração se torna clara ao observar que poucos produtos, famílias de produtos, planos de pagamentos e cidades de consumo se destacam no tamanho e cor, ao fazer a análise gráfica de cada uma das análises da seção (Figuras 10, 12 , $13,15,17,19,21,22$ e 26$)$.

\subsubsection{Definição das ofertas}

A partir da análise gráfica das associações, escolheu-se produtos de seis das dezessete famílias de produtos, para o desenho de uma oferta dirigida aos clientes nas simulações para aplicação do modelo de segmentação.

As ofertas a serem comunicadas de forma simulada à base de clientes estão relacionadas na Tabela 37. A partir destas ofertas determinou-se, para cada mês observado, seis segmentos de clientes, de forma a cada um receber a comunicação de uma oferta. 


\begin{tabular}{|c|c|c|c|c|}
\hline $\begin{array}{l}\text { Número da } \\
\text { Oferta } \\
\text { (Segmento) }\end{array}$ & Descrição da oferta & $\begin{array}{l}\text { Família de } \\
\text { produtos }\end{array}$ & $\begin{array}{l}\text { Plano de } \\
\text { Pagamento }\end{array}$ & $\begin{array}{l}\text { Região da } \\
\text { Oferta }\end{array}$ \\
\hline 1 & $\begin{array}{l}\text { Lavagem automotiva por } \mathrm{R} \$ \\
25,00\end{array}$ & $\begin{array}{l}\text { Posto de } \\
\text { Gasolina }\end{array}$ & À vista & Salvador \\
\hline 2 & $\begin{array}{l}\text { Compra pela internet de } \\
\text { perfumes importados por } R \$ \\
100,00\end{array}$ & Perfumaria & $\begin{array}{l}\text { De } 3 x \text { a } 6 x \\
\text { sem juros }\end{array}$ & Brasil \\
\hline 3 & $\begin{array}{l}\text { Jantar para um casal por } \mathrm{R} \$ \\
38,00\end{array}$ & Alimentos & A vista & Olinda \\
\hline 4 & $\begin{array}{l}\text { Par de brincos banhado a } \\
\text { ouro por } \mathrm{R} \$ 75,00\end{array}$ & Joalheria & $\begin{array}{l}\text { Até } 12 \mathrm{x} \text { sem } \\
\text { juros }\end{array}$ & Brasil \\
\hline 5 & $\begin{array}{l}\text { Lavagem de vestido por } \mathrm{R} \$ \\
45,00\end{array}$ & Serviços & A vista & São Paulo \\
\hline 6 & $\begin{array}{l}\text { Compra pela internet de } \\
\text { seriados em DVD por } R \$ \\
90,00\end{array}$ & Livraria & $\begin{array}{l}\text { Até } 12 x \text { sem } \\
\text { juros }\end{array}$ & Brasil \\
\hline
\end{tabular}

Tabela 37: Ofertas para a determinação dos segmentos de clientes

\subsubsection{Seleção das regras de associação relevantes}

Considerando-se a mesma regra utilizada na exemplificação do método para seleção das regras para a seleção do público e posterior realização da comunicação da oferta (Seção 5.4), inicia-se a busca das regras a partir das transações consequentes que coincidem com a oferta que se quer comunicar aos clientes.

O segundo passo é a seleção das compras antecedentes mais frequentes, e a formação de um conjunto de regras de seleção de clientes. O critério fundamental para a utilização das regras na segmentação é que o lift calculado para a regra seja superior a 2.

\subsubsection{Algoritmo de segmentação de clientes (ASC)}

Uma vez definidas as ofertas a se comunicar aos clientes (Tabela 37), um conjunto de expressões lógicas semelhantes à de número (8) determinarão quais são os clientes que farão a composição de cada segmento.

Com base nas seis ofertas a serem comunicadas, realizou-se a separação das regras para a segmentação, resultando em: 1.010 regras apontadas pela ACC descrita na Seção 5.3; 2070 regras apontadas pelas sete análises descritas nas 
Seções 5.2.1; 5.2.2; 5.2.3; 5.2.4; 5.2.5; 5.2 .6 e 5.2.8. A análise descrita na Seção 5.2.7 não contribuiu com nenhuma regra, pois todas as regras de associação encontradas demonstravam-se pouco eficientes com o maior lift inferior a 2.

Na Expressão 9, demonstra-se o ASC de cartão de crédito baseado nas transações dos clientes. Os agrupamentos de clientes se darão a partir da execução do algoritmo, fazendo o uso das regras demonstradas nas Tabelas 47 e 48 do Apêndice 2.

$\{[$ Clientes que tenham comprado no mês

(Regras de produtos e taxonomia da ACC)

OU clientes que tenham comprado no mês

(Regras de família de produto da AA no mês anterior)

OU clientes que tenham comprado no mês

(Regras de produto da AA no mês anterior)]

E clientes que tenham comprado no mês

(Regras de plano de pagamento da AA no mês anterior)

E clientes que tenham comprado no mês

(Regras de cidades de realização da compra da AA no mês anterior)\}

OU

$\{[$ Clientes que tenham comprado nos últimos 12 meses

(Regras de família de produto da AA no mês anterior)

OU clientes que tenham comprado no nos últimos 12 meses

(Regras de produto da AA no mês anterior)]

E clientes que tenham comprado no nos últimos 12 meses

(Regras de cidades de realização da compra da AA no mês anterior)\}

\subsubsection{Aplicação do ASC}

A base de clientes que terá a aplicação do ASC é a base de clientes que possuem faturas na amostra de validação. A amostra de validação, cujas transações não foram utilizadas nas análises descritas nas Seções 5.2 e 5.3, é uma base de 42.917 faturas de 20.509 clientes e compreendem 196.373 compras realizadas no período do estudo. 
Observa-se na Tabela 38 a quantidade de clientes da base de validação para cada mês, a partir do terceiro mês do período estudado.

\begin{tabular}{cc}
\hline Mês observado & Clientes \\
\hline 3 & 3.600 \\
4 & 3.642 \\
5 & 3.651 \\
6 & 3.480 \\
7 & 3.559 \\
8 & 3.532 \\
9 & 3.358 \\
10 & 3.611 \\
11 & 3.465 \\
12 & 4.038
\end{tabular}

Tabela 38: Clientes por mês de observação na amostra de validação

O ASC será aplicado sobre a base das compras dos clientes, realizadas antes das transações da amostra de validação.

Para cada conjunto de clientes observados, tem-se a segmentação para comunicação das ofertas e, como resultado, apresentam-se seis segmentos de clientes para cada mês de observação. Cada segmento se caracteriza pelo número da oferta selecionada para os clientes, presente na Tabela 37.

\begin{tabular}{ccccccc}
\hline $\begin{array}{c}\text { Mês } \\
\text { Observado }\end{array}$ & 1 & 2 & 3 & 4 & 5 & 6 \\
\hline 3 & 136 & 2.252 & 647 & 1.882 & 261 & 2.060 \\
4 & 156 & 2.642 & 766 & 2.272 & 326 & 2.460 \\
5 & 184 & 2.875 & 772 & 2.510 & 311 & 2.703 \\
6 & 182 & 2.849 & 864 & 2.547 & 385 & 2.718 \\
7 & 191 & 2.998 & 925 & 2.662 & 364 & 2.859 \\
8 & 224 & 3.060 & 956 & 2.754 & 420 & 2.937 \\
9 & 214 & 3.002 & 934 & 2.729 & 391 & 2.892 \\
10 & 230 & 3.255 & 990 & 2.968 & 421 & 3.136 \\
11 & 240 & 3.127 & 1.024 & 2.899 & 461 & 3.034 \\
12 & 285 & 3.647 & 1.215 & 3.377 & 565 & 3.542 \\
\hline
\end{tabular}

Tabela 39: Quantidade de clientes por segmento a cada mês observado 
Para cada segmento e mês observados, a quantidade de clientes está expressa na Tabela 39 na qual se apurou 99.688 comunicações como o total resultante da segmentação para os seis produtos selecionados.

Esta tabela servirá de base para a apuração dos ganhos de eficiência na comunicação das ofertas a estes clientes.

5.5.5 Transações da base de validação.

O levantamento das quantidades de clientes que realizaram as transações relativas às ofertas 1 a 6 se demonstra na Tabela 40 e totaliza 919 transações.

Segmentar a base de clientes antes que estas transações ocorram a fim de encaminhar o menor número possível de comunicações é o objetivo desta pesquisa, cujos resultados serão apresentados na próxima seção.

\begin{tabular}{ccccccc}
\hline $\begin{array}{c}\text { Mês } \\
\text { Observado }\end{array}$ & 1 & 2 & 3 & 4 & 5 & 6 \\
\hline 3 & 17 & 13 & 1 & 34 & 1 & 23 \\
4 & 17 & 13 & 2 & 33 & 3 & 22 \\
5 & 13 & 19 & 3 & 29 & 3 & 12 \\
6 & 25 & 19 & 1 & 22 & 0 & 22 \\
7 & 19 & 8 & 1 & 34 & 2 & 11 \\
8 & 24 & 22 & 1 & 21 & 2 & 16 \\
9 & 17 & 18 & 1 & 35 & 1 & 7 \\
10 & 26 & 24 & 0 & 28 & 2 & 13 \\
11 & 18 & 22 & 3 & 39 & 1 & 16 \\
12 & 19 & 27 & 4 & 66 & 4 & 20 \\
\hline
\end{tabular}

Tabela 40: Clientes que realizaram as transações oferecidas

Sem a aplicação da segmentação de clientes, a comunicação de todas as ofertas seria direcionada a todo o público, o que totalizaria 257.502 mensagens oferecendo um produto cada aos clientes.

Para se ter a dimensão inicial da eficiência de comunicação, calculou-se a quantidade de comunicações para cada cliente que realizou a compra referente à 
oferta de cada segmento. Os valores da eficiência da comunicação, levando-se em consideração as transações da amostra de validação, encontram-se na Tabela 41.

\begin{tabular}{cclllll}
\hline $\begin{array}{c}\text { Mês } \\
\text { Observado }\end{array}$ & 1 & 2 & 3 & 4 & 5 & 6 \\
\hline 3 & 207 & 270 & 3.512 & 103 & 3.512 & 153 \\
4 & 204 & 267 & 1.735 & 105 & 1.156 & 158 \\
5 & 277 & 189 & 1.200 & 124 & 1.200 & 300 \\
6 & 146 & 192 & 3.642 & 166 & - & 166 \\
7 & 192 & 456 & 3.651 & 107 & 1.826 & 332 \\
8 & 145 & 158 & 3.480 & 166 & 1.740 & 218 \\
9 & 209 & 198 & 3.559 & 102 & 3.559 & 508 \\
10 & 136 & 147 & - & 126 & 1.766 & 272 \\
11 & 187 & 153 & 1.119 & 86 & 3.358 & 210 \\
12 & 190 & 134 & 903 & 55 & 903 & 181 \\
\hline
\end{tabular}

Tabela 41: Eficiência de comunicação sem segmentação

Comunicar a todos os clientes sobre todos os produtos resultaria em uma comunicação com resultado positivo a cada 280 comunicações, aproximadamente. 


\section{RESULTADOS}

Os resultados obtidos por meio do modelo de segmentação de clientes por perfil transacional são demonstrados a partir do cálculo de ganho de eficiência na comunicação. Para cada segmento testado são expressas duas medidas: a eficiência da comunicação e a cobertura da oferta.

Como resultado da intersecção dos conjuntos de clientes segmentados a cada mês com os clientes que, de fato, realizaram as transações esperadas, tem-se 691 transações. Apresentam-se, na Tabela 42, as quantidades de transações por segmento (descritos na Tabela 37) e por mês de observação.

\begin{tabular}{ccccccc}
\hline Mês & \multicolumn{7}{c}{ Segmentos } \\
\cline { 2 - 7 } Observado & 1 & 2 & 3 & 4 & 5 & 6 \\
\hline 3 & 2 & 12 & 1 & 24 & 0 & 15 \\
4 & 4 & 10 & 2 & 24 & 0 & 16 \\
5 & 4 & 14 & 3 & 26 & 1 & 11 \\
6 & 8 & 16 & 1 & 20 & 0 & 20 \\
7 & 8 & 7 & 1 & 29 & 0 & 9 \\
8 & 11 & 22 & 1 & 20 & 0 & 14 \\
9 & 5 & 18 & 1 & 31 & 0 & 7 \\
10 & 7 & 24 & 0 & 27 & 0 & 12 \\
11 & 7 & 22 & 3 & 36 & 0 & 16 \\
12 & 9 & 27 & 4 & 60 & 0 & 19 \\
\hline Tabela 42: Clientes & que receberam a comunicação & e realizaram a \\
transação oferecida. & \multicolumn{7}{c}{}
\end{tabular}

\subsection{Eficiência na comunicação}

A partir da simulação do envio de 99.680 comunicações de ofertas para os clientes - demonstrado na Seção 5.5.4 - que teria apurado 691 ofertas bem sucedidas - demonstrado na Seção 6 - conclui-se que o ASC teve um resultado positivo a cada 144 comunicações, aproximadamente.

Ao comparar a situação inicial, demonstrada na Seção 5.5.5 - na qual havia a necessidade de se realizar 280 comunicações para atingir um cliente que viesse a 
efetuar uma transação esperadas - com a aplicação do ASC a quantidade de comunicações para cada transação esperada reduziu em $48,6 \%$.

Ao verificar a eficiência simulada da comunicação aos clientes dos seis segmentos, percebe-se que alguns segmentos apresentaram elevada eficiência.

A Tabela 43 apresenta a graduação de tom indicando com verde escuro, as combinações de segmento e mês observado com maior ganho de eficiência na comunicação. As células da tabela com preenchimento "-" (missing), representam combinações de segmento e mês de observação nos quais nenhuma comunicação atingiu clientes que efetuaram as transações esperadas.

\begin{tabular}{ccccccc}
\hline $\begin{array}{c}\text { Mês } \\
\text { Observado }\end{array}$ & 1 & 2 & 3 & 4 & 5 & 6 \\
\hline 3 & 68 & 188 & 647 & 78 & - & 137 \\
4 & 39 & 264 & 383 & 95 & - & 154 \\
5 & 46 & 205 & 257 & 97 & 311 & 246 \\
6 & 23 & 178 & 864 & 127 & - & 136 \\
7 & 24 & 428 & 925 & 92 & - & 318 \\
8 & 20 & 139 & 956 & 138 & - & 210 \\
9 & 43 & 167 & 934 & 88 & - & 413 \\
10 & 33 & 136 & - & 110 & - & 261 \\
11 & 34 & 142 & 341 & 81 & - & 190 \\
12 & 32 & 135 & 304 & 56 & - & 186 \\
\hline
\end{tabular}

Tabela 43: Eficiência da comunicação com segmentação

Ao relativizar os dados de comunicação com os dados da eficiência da comunicação sem segmentação (Tabela 41), ratificam-se os destaques atribuídos à Tabela 43. Pode-se observar reduções de custo com comunicação entre 60\% e $80 \%$ para os segmentos de oferta 1 (Lavagem automotiva) e 3 (Jantar para um casal) enquanto os demais segmentos de oferta tiveram a redução de custo relativa inferior a $20 \%$ na maioria dos casos.

A Tabela 44 exibe doze situações, das quais 9 para a oferta do segmento 5 (Lavagem de vestido), nas quais se apresentou aumento do custo de comunicação 
relativo, ou seja, uma proporção maior de comunicações por retorno bem sucedido foi encaminhada ao cliente.

\begin{tabular}{ccccccc}
\hline Mês & \multicolumn{7}{c}{ Segmentos } \\
\cline { 2 - 7 } Observado & 1 & 2 & 3 & 4 & 5 & 6 \\
\hline 3 & $67 \%$ & $31 \%$ & $82 \%$ & $24 \%$ & - & $10 \%$ \\
4 & $81 \%$ & $1 \%$ & $78 \%$ & $10 \%$ & - & $2 \%$ \\
5 & $83 \%$ & $-8 \%$ & $79 \%$ & $22 \%$ & $74 \%$ & $18 \%$ \\
6 & $84 \%$ & $7 \%$ & $76 \%$ & $23 \%$ & - & $18 \%$ \\
7 & $88 \%$ & $6 \%$ & $75 \%$ & $15 \%$ & - & $4 \%$ \\
8 & $86 \%$ & $12 \%$ & $73 \%$ & $17 \%$ & - & $4 \%$ \\
9 & $80 \%$ & $16 \%$ & $74 \%$ & $13 \%$ & - & $19 \%$ \\
10 & $76 \%$ & $8 \%$ & - & $13 \%$ & - & $4 \%$ \\
11 & $82 \%$ & $7 \%$ & $70 \%$ & $6 \%$ & - & $10 \%$ \\
12 & $83 \%$ & $-1 \%$ & $66 \%$ & $-3 \%$ & - & $-3 \%$ \\
\hline
\end{tabular}

Tabela 44: Redução de custo relativo com a segmentação

No entanto com exceção do segmento de número 5 , os demais segmentos apresentaram redução relativa de custo de comunicação, levando em consideração o período total observado.

O Gráfico 20 demonstra a redução de custo relativo de comunicação, uma vez realizada a segmentação segundo o ASC.

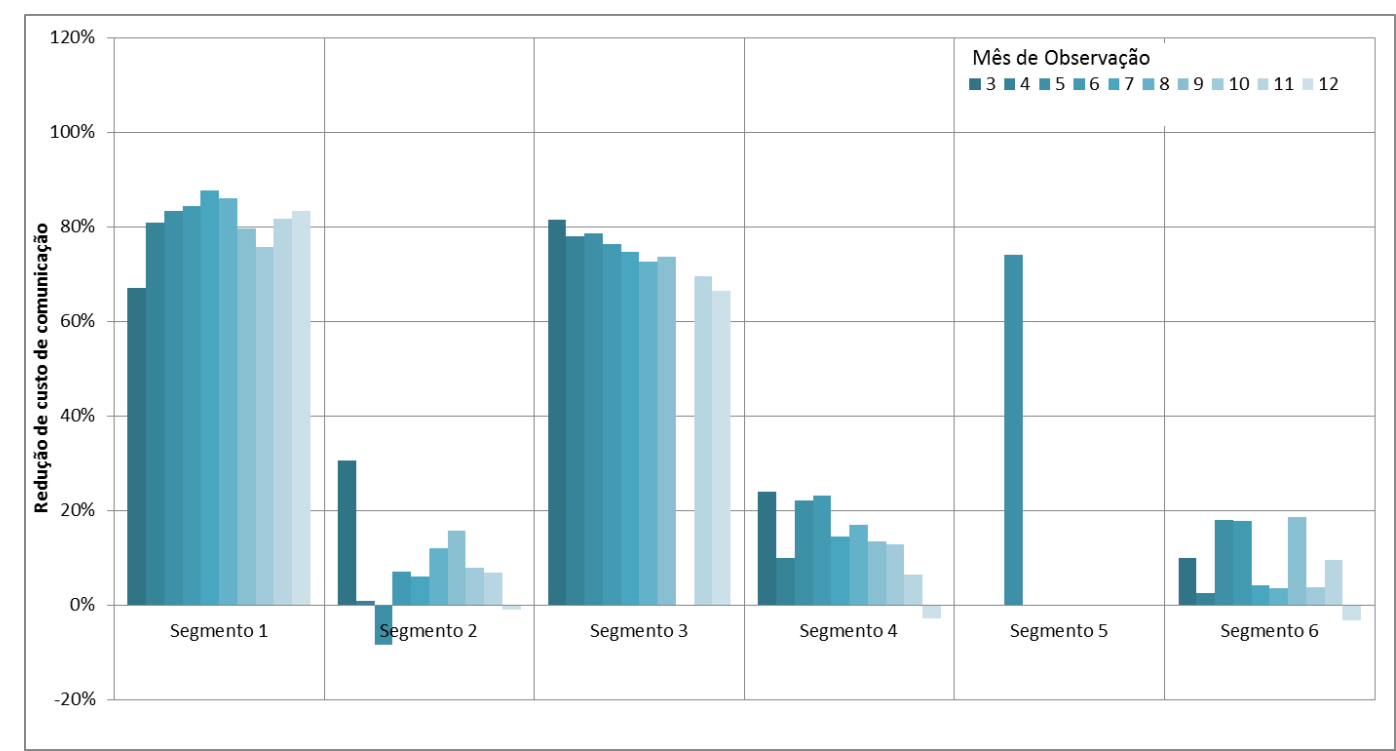

Gráfico 20: Redução de custo de comunicação relativo com segmentação 
Apresentam-se no Gráfico 21 os ganhos de eficiência da aplicação do ASC para cada segmento a cada mês do período estudado.

A partir da análise do gráfico, percebe-se elevados ganhos provenientes da aplicação do ASC para os segmentos 1, 3 e 5 - sendo o último pontual em apenas um mês do estudo - enquanto que nos segmentos 2, 4 e 6 este ganho é reduzido.

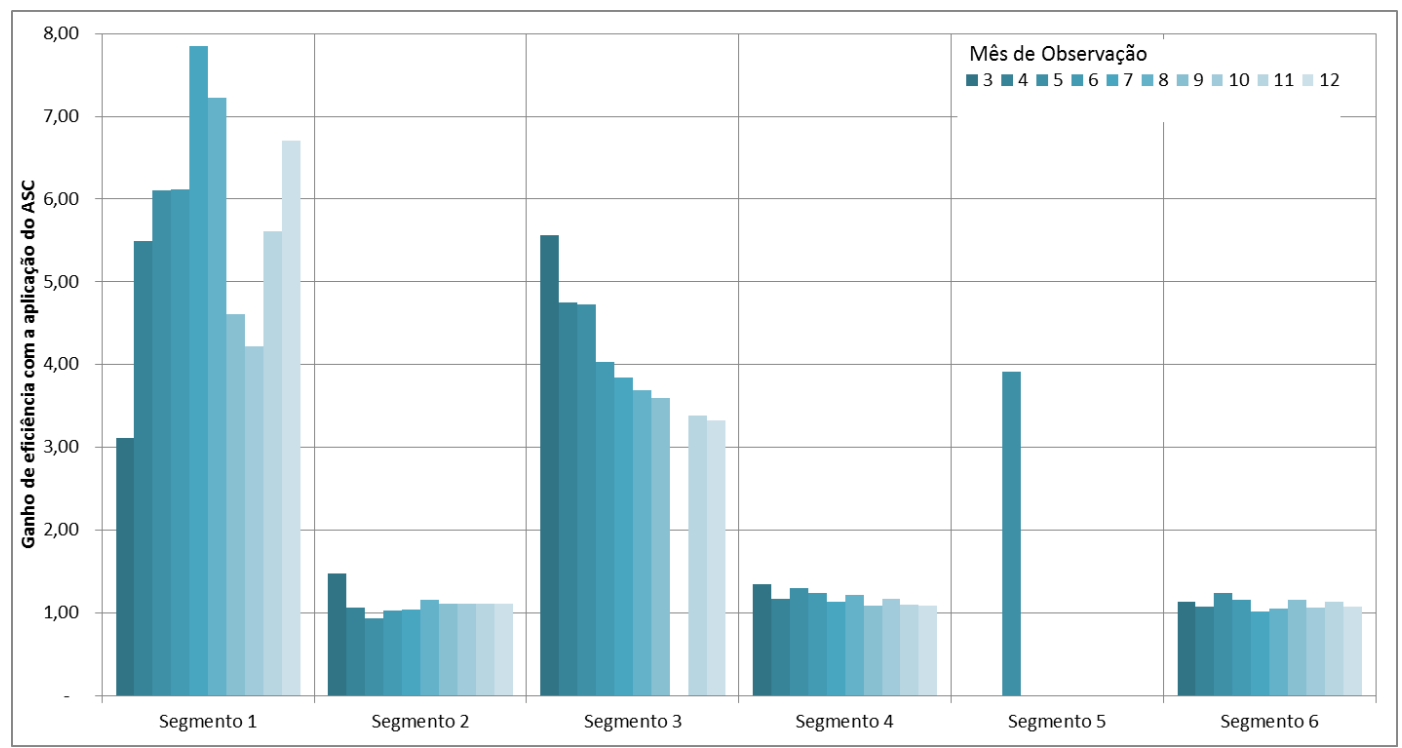

Gráfico 21: Ganho de eficiência de comunicação com segmentação

Embora o lift de todas as regras que compõem a expressão lógica de cada segmento seja no mínimo 2, percebe-se que o ganho de eficiência dos segmentos 2 , 4 e 6 são pouco superiores a 1, apresentando-se inferior a 1 em alguns casos: mês 5 no segmento 2; mês 10 no segmento 3 ; meses $3,4,6,7,8,9,10,11,12$ no segmento 5 .

A razão provável para os casos cujos ganhos de eficiência se apresentaram inferior a 1 provém do fato da base de transações que fora utilizada na investigação das regras de segmentação ser diferente da amostra de validação. Desta forma, a simulação representa a realidade.

A alteração do critério de seleção das regras de composição da expressão lógica de cada segmento para aperfeiçoar o processo de segmentação não está prevista neste trabalho, mas pode perfeitamente ser abordada em pesquisas futuras. 


\subsection{Cobertura da oferta}

Ao segmentar o público, de acordo com as transações realizadas para ganhar eficiência no custo de comunicação, também se opta por atingir apenas parte dos clientes potenciais com a comunicação dirigida.

A Tabela 45 apresenta a cobertura de cada uma das ofertas ao longo do período de observação do estudo. A ênfase é dada pela coloração verde. Quanto mais escura, maior parte - ou mesmo o total - das compras dos produtos oferecidos foi realizada por clientes que compunham o segmento de oferta.

\begin{tabular}{ccccccc}
\hline $\begin{array}{c}\text { Mês } \\
\text { Observado }\end{array}$ & 1 & 2 & 3 & 4 & 5 & 6 \\
\hline 3 & $12 \%$ & $92 \%$ & $100 \%$ & $71 \%$ & $0 \%$ & $65 \%$ \\
4 & $24 \%$ & $77 \%$ & $100 \%$ & $73 \%$ & $0 \%$ & $73 \%$ \\
5 & $31 \%$ & $74 \%$ & $100 \%$ & $90 \%$ & $33 \%$ & $92 \%$ \\
6 & $32 \%$ & $84 \%$ & $100 \%$ & $91 \%$ & $0 \%$ & $91 \%$ \\
7 & $42 \%$ & $88 \%$ & $100 \%$ & $85 \%$ & $0 \%$ & $82 \%$ \\
8 & $46 \%$ & $100 \%$ & $100 \%$ & $95 \%$ & $0 \%$ & $88 \%$ \\
9 & $29 \%$ & $100 \%$ & $100 \%$ & $89 \%$ & $0 \%$ & $100 \%$ \\
10 & $27 \%$ & $100 \%$ & $0 \%$ & $96 \%$ & $0 \%$ & $92 \%$ \\
11 & $39 \%$ & $100 \%$ & $100 \%$ & $92 \%$ & $0 \%$ & $100 \%$ \\
12 & $47 \%$ & $100 \%$ & $100 \%$ & $91 \%$ & $0 \%$ & $95 \%$ \\
\hline
\end{tabular}

Tabela 45: Cobertura das ofertas

O Gráfico 22, que apresenta a cobertura da oferta por segmento, por mês observado, demonstra a diferença nos níveis de cobertura atingidos para cada segmento.

Os segmentos de números 2, 3, 4 e 6, tiveram uma cobertura de oferta bastante elevada, atingindo, por vezes, $100 \%$ dos clientes que realizaram a transação. Entretanto, os segmentos de oferta 1 e 5 apresentaram a cobertura de oferta baixa, atingindo, nos melhores meses patamares inferiores a $50 \%$ das transações no segmento 1 e $33 \%$ apenas em um mês para o segmento 5 . 


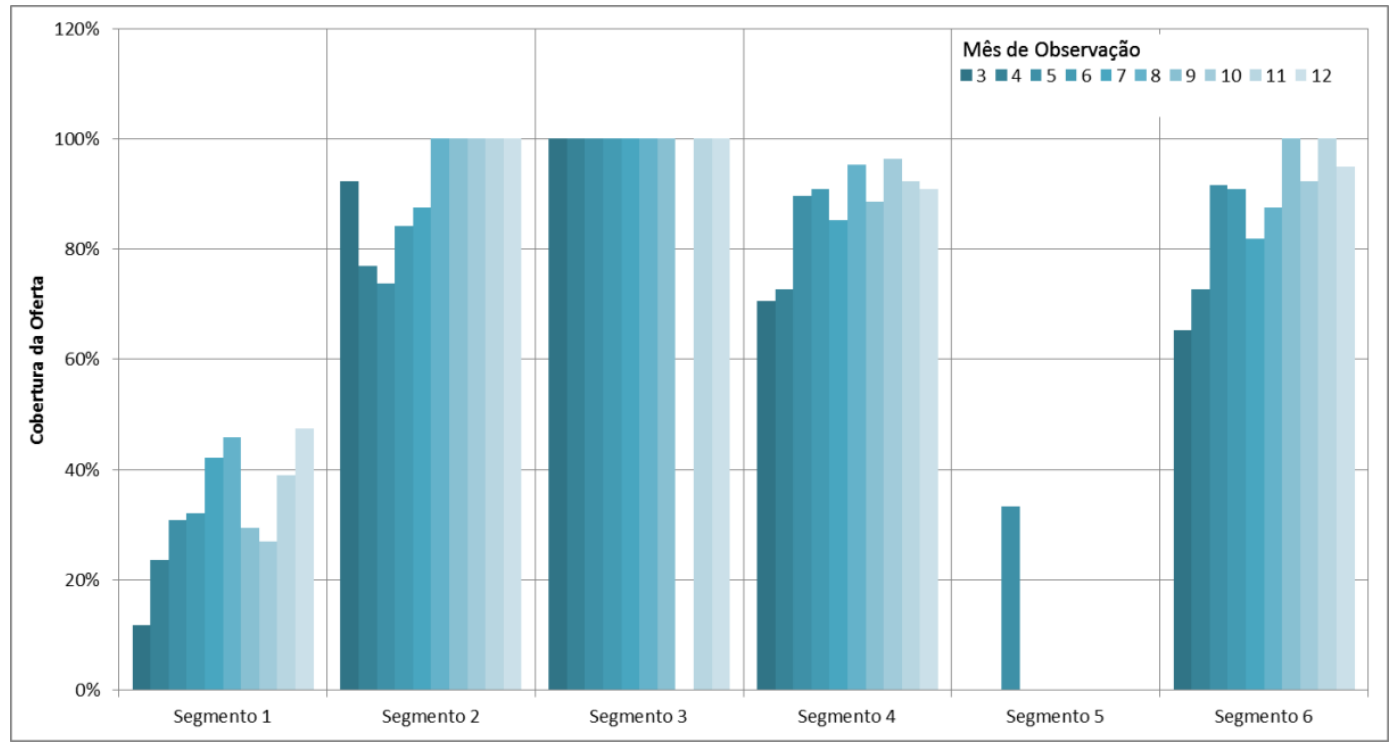

Gráfico 22: Cobertura da oferta segmentada

O segmento de oferta 5 não demonstrou cobertura que justifique a sua existência, considerando o ASC estudado.

\subsection{Equilíbrio entre eficiência e cobertura}

As medidas de eficiência e cobertura das ofertas são úteis na busca do equilíbrio que permita a redução de custo com comunicação, sem que as vendas sejam significativamente reduzidas.

Ao analisar o segmento 1, nota-se que, com a aplicação do ASC sobre a base de clientes, apenas 2.042 clientes ao longo do ano foram selecionados para receber a oferta $(4,8 \%$ do total). 65 clientes selecionados realizaram a compra esperada, resultando em uma eficiência de 31,4 comunicações para cada sucesso. O total de vendas de lavagens automotivas no período e região foi de 195, resultando em uma cobertura de oferta de 33,3\% e um ganho de eficiência de 7 vezes.

$\mathrm{Na}$ análise do segmento 2, nota-se que, 29.707 clientes foram selecionados para receber a oferta (69,2\% do total), atingindo 172 comunicações bem sucedidas (eficiência de 172,7 comunicações por sucesso e 1,3 vezes de ganho). O total de vendas de perfumes pela internet no período foi de, 185 , resultando em $93,0 \%$ de cobertura de oferta. 
A aplicação do ASC para o segmento de número 3 apresentou $100 \%$ de cobertura, ou seja, os 17 clientes que consumiram jantares em Olinda, receberam a comunicação na simulação da pesquisa. A eficiência deste segmento foi de 534,9 comunicações por sucesso (ganho de 4,7 vezes) e atingiu $21,19 \%$ dos clientes potenciais (9.093).

Ao analisar o segmento 4, 26.600 clientes ao longo do ano foram selecionados para receber a oferta $(62,0 \%$ do total). 297 clientes selecionados realizaram a compra nas joalherias do produto oferecido, resultando em uma eficiência de 89,6 comunicações para cada sucesso cujo ganho representa 1,4 vezes. O total de vendas destes brincos no período foi de 341 , resultando em uma cobertura de oferta de $87,1 \%$.

O segmento 5 ilustra o caso extremo, onde a oferta foi muito restrita. Com a aplicação do ASC, apenas 3.905 clientes $(9,1 \%$ do total) foram selecionados para receber a oferta e destes, apenas 1 realizou a compra (eficiência de 3.905 comunicações para uma comunicação de sucesso) resultando em perda de eficiência de 0,6 vezes. Outras 18 transações do produto em questão ocorreram, mas não foram capturadas pelos segmentos comunicados, resultando em uma cobertura de $5,3 \%$.

Por fim, a aplicação do ASC para o segmento de número 6, com a oferta de vendas de DVDs pela internet, demonstrou $85,8 \%$ de cobertura, com eficiência de 203,9 comunicações por sucesso (ganho de 1,3 vezes), atingindo $66,0 \%$ dos clientes potenciais $(28.341)$.

A Tabela 46 apresenta o resumo dos resultados da aplicação do ASC aos clientes estudados, com destaque em verde para os acertos da segmentação e em vermelho para os erros.

A terceira coluna da tabela divide os clientes entre os que compraram e não compraram os produtos oferecidos a cada um dos segmentos. A quarta e a quinta colunas da tabela representam as quantidades de transações alvo da segmentação que ocorreram, respectivamente, dentro ou fora de seus segmentos. 
Desta forma pode-se avaliar para quais segmentos a oferta foi mais efetiva, ao utilizar o modelo de comunicação segmentada pelo ASC.

\begin{tabular}{|c|c|c|c|c|c|c|c|c|}
\hline Oferta & $\begin{array}{l}\text { Descrição } \\
\text { da oferta }\end{array}$ & Retorno & $\begin{array}{l}\frac{\pi}{ \pm} \\
\frac{1}{0} \\
\frac{0}{0} \\
0 \\
0 \\
0 \\
0 \\
\frac{1}{0} \\
0 \\
\frac{1}{0} \\
0 \\
0 \\
0 \\
Z\end{array}$ & 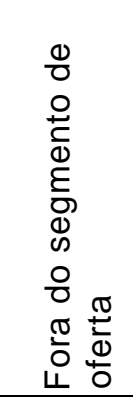 & 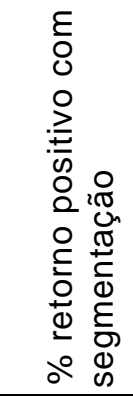 & 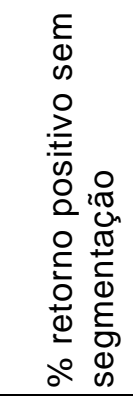 & 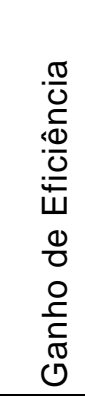 & $\begin{array}{l}\frac{\pi}{3} \\
\frac{\pi}{0} \\
\frac{0}{0} \\
0\end{array}$ \\
\hline \multirow[b]{2}{*}{1} & \multirow{2}{*}{$\begin{array}{l}\text { Lavagem } \\
\text { automotiva } \\
\text { por } \mathrm{R} \$ \\
25,00\end{array}$} & Comprou & 65 & 130 & \multirow[b]{2}{*}{$3,18 \%$} & \multirow[b]{2}{*}{$0,45 \%$} & \multirow[b]{2}{*}{7,0} & \multirow[b]{2}{*}{$33,3 \%$} \\
\hline & & $\begin{array}{l}\text { Não } \\
\text { Comprou }\end{array}$ & 1.977 & 40.745 & & & & \\
\hline \multirow[b]{2}{*}{2} & \multirow{2}{*}{$\begin{array}{l}\text { Compra } \\
\text { pela internet } \\
\text { de perfumes } \\
\text { importados } \\
\text { por } R \$ \\
100,00\end{array}$} & Comprou & 172 & 13 & \multirow[b]{2}{*}{$0,58 \%$} & \multirow[b]{2}{*}{$0,43 \%$} & \multirow[b]{2}{*}{1,3} & \multirow[b]{2}{*}{$93,0 \%$} \\
\hline & & $\begin{array}{l}\text { Não } \\
\text { Comprou }\end{array}$ & 29.535 & 13.197 & & & & \\
\hline \multirow[b]{2}{*}{3} & \multirow{2}{*}{$\begin{array}{l}\text { Jantar para } \\
\text { um casal } \\
\text { por } R \$ \\
38,00\end{array}$} & Comprou & 17 & - & \multirow[b]{2}{*}{$0,19 \%$} & \multirow[b]{2}{*}{$0,04 \%$} & \multirow[b]{2}{*}{4,7} & \multirow[b]{2}{*}{$100,0 \%$} \\
\hline & & $\begin{array}{l}\text { Não } \\
\text { Comprou }\end{array}$ & 9.076 & 33.824 & & & & \\
\hline \multirow[b]{2}{*}{4} & \multirow{2}{*}{$\begin{array}{l}\text { Par de } \\
\text { brincos } \\
\text { banhado a } \\
\text { ouro por } \mathrm{R} \$ \\
75,00\end{array}$} & Comprou & 297 & 44 & \multirow[b]{2}{*}{$1,12 \%$} & \multirow[b]{2}{*}{$0,79 \%$} & \multirow[b]{2}{*}{1,4} & \multirow[b]{2}{*}{$87,1 \%$} \\
\hline & & $\begin{array}{l}\text { Não } \\
\text { Comprou }\end{array}$ & 26.303 & 16.273 & & & & \\
\hline \multirow[b]{2}{*}{5} & \multirow{2}{*}{$\begin{array}{l}\text { Lavagem de } \\
\text { vestido por } \\
\mathrm{R} \$ 45,00\end{array}$} & Comprou & 1 & 18 & \multirow[b]{2}{*}{$0,03 \%$} & \multirow[b]{2}{*}{$0,04 \%$} & \multirow[b]{2}{*}{0,6} & \multirow[b]{2}{*}{$5,3 \%$} \\
\hline & & $\begin{array}{l}\text { Não } \\
\text { Comprou }\end{array}$ & 3.904 & 38.994 & & & & \\
\hline \multirow[b]{2}{*}{6} & \multirow{2}{*}{$\begin{array}{l}\text { Compra } \\
\text { pela internet } \\
\text { de seriados } \\
\text { em DVD por } \\
R \$ 90,00\end{array}$} & Comprou & 139 & 23 & \multirow[b]{2}{*}{$0,49 \%$} & \multirow[b]{2}{*}{$0,38 \%$} & \multirow[b]{2}{*}{1,3} & \multirow[b]{2}{*}{$85,8 \%$} \\
\hline & & $\begin{array}{l}\text { Não } \\
\text { Comprou }\end{array}$ & 28.202 & 14.553 & & & & \\
\hline
\end{tabular}

Tabela 46: Resumo dos resultados da aplicação do ASC

Os valores dos percentuais de retorno positivos representam o percentual de clientes que aceitaram a oferta de determinada comunicação segmentada ou para todos os clientes. A partir da relação do retorno positivo com e sem segmentação de público, apura-se o ganho de eficiência. 
A cobertura da oferta, já discutida na Seção 6.2 representa o percentual de clientes que consumiram os produtos oferecidos e que participavam dos segmentos de oferta.

Como conclusão da interpretação desta tabela, tem-se que apenas 0 segmento de número 5 apresentou redução da eficiência, encarecendo a comunicação. Desta forma, conclui-se que para a maioria dos casos que se queira segmentar, o ASC contribui com a determinação das ofertas apresentando ganhos de eficiência para a empresa que dele se utilizar. 


\section{CONCLUSÃO}

Como principal objetivo da segmentação, a assertividade no relacionamento com o cliente usuário de cartão de crédito pode ser incrementada baseando-se no histórico de transações realizadas.

A pesquisa indica que a aplicação de análise de cesto de compras e a análise de associação às bases transacionais pode aumentar a eficiência da comunicação com o cliente. Em termos práticos, este ganho de eficiência tende a se reverter em investimentos para retroalimentar a indústria de cartões de crédito. Parte destes ganhos pode ser aplicada na manutenção dos clientes, materializando-se por meio de ofertas de consumo mais competitivas.

Por meio das técnicas de análise de cesto de compras e análise de associação houve a elaboração e aplicação de modelos de segmentação de clientes de cartão de crédito.

Os modelos identificaram cinco classes de ofertas a serem comunicadas de forma efetiva, apresentando ganhos na eficiência da comunicação destas ofertas.

Não está no escopo desta esta pesquisa estimar se estes ganhos são suficientes para causar alguma mudança significativa no mercado de cartões de crédito, mas os resultados aqui exibidos vêm ao encontro da intensão da pesquisa.

Dado que o tratamento de grupos homogêneos de clientes possibilita a oferta de produtos e serviços adequados às demandas dos consumidores, o caminho da segmentação do cliente de cartão de crédito a partir dos dados transacionais se demonstra efetivo. 


\section{BIBLIOGRAFIA}

AGRAWAL, R.; SRIKANT, R. Fast Algorithms for Mining Associations Rules. IBM Almaden Research Center, p. 3-16, 1994.

BERNTHAL, M. J.; CROCKETT, D.; ROSE, R. L. Credit Cards as Lifestile Facilitator. Journal of Consumer Research, v. 32, 2005.

BLACKWELL, R. D.; MINIARD, P. W.; ENGEL, J. F. Consumer Behavior. 9ed. ed. Ohio: South-Western Thomson Learning, 2002.

BLATTBERG, R. C.; SEN, S. K. Market Segmentation Using Models of Multidimensional Purchasing Behavior. The Journal of Marketing, v. 38, p. 17-28, Oct 1974.

BLATTBERG, R. et al. Identifying the Deal Prone Segment. Journal of Marketing Research, v. 15, n. American Marketing Association, p. 369-377, Aug 1978.

BRIN, S. et al. Dynamic Itemset Counting and Implication Rules for MArket Basket Data. Stanford University, p. 1-10, 1997.

GARCIA, G. Credit Cards: An Interdisciplinary Survey. Journal of Consumer Research, v. vol 6, 1980.

GROVER, R.; SRINIVASAN, V. A Simultaneous Approach to Market Segmentation and Market Structuring. Journal of Marketing Research, v. 24, p. 139-153, May 1987.

GUHA, S.; RASTOGI, R.; SHIM, K. ROCK: A Robust Clustering Algorithm for Categorical Attributes. Information Systems, v. 25, n. 5, p. 345-366, 2000.

HAYHOE, C. R. et al. Differences in spending habits and credit use of college students. The Journal of Consumer Affairs., v. vol 34, 2000. 
HOCH, S. J.; LOEWENSTEIN, G. F. Time-Inconsistent Preferences and Consumer Self-Control. Journal of Consumer Research., v. Vol. 17., 1991.

HOCK, D. Nascimento da era caórdica. 1. ed. São Paulo: Cultrix, 2000.

HOFFMAN, K. D. et al. Princípios de Marketing de Serviços. São Paulo: CENGAGE Learning, 2009. tradução da $3^{\underline{a}}$ ed. Americana.

KAMAKURA, W. A.; RUSSEL, G. J. A Probabilistic Choice Model for Market Segmentation and Elasticity Structu. Journal of Marketing Research, v. 26, p. 379390, Nov 1989.

KIM, S. Y. et al. Customer segmentation and strategy development based on customer lifetime value: A case study. Expert systems with application, v. 31, p. 101-107, 2006.

KOTLER, P. Administração de Marketing. 10. ed. São Paulo: Prentice Hall, 2000.

LEE, J. H.; PARK, S. C. Intelligent profitable customers segmentation system based on business intelligence tools. Expert systems with application, v. 29, p. 145-152, 2005.

LEVINE, D. M.; BERENSON, M. L.; STEPHAN, D. Estatística: Teoria e Aplicações. Rio de Janeiro: LTC, 1988.

LINOFF, G. S.; BERRY, M. J. A. Data Mining Techniques for Marketing, Sales and Customer Relationship Management. 2. ed. Hoboken: John Wiley \& Sons, 2011.

LOHR, S. L. Sampling: Design and Analysis. Pacific Grove: Duxbury Press, 1999. 494 p. 
LOVELOCK, C.; WRIGHT, L. Serviços: Marketing e Gestão. São Paulo: Saraiva, 2002.

O'BRIEN, J. A. Sistemas de Informação e as Decisões Gerenciais na Era da Internet. Tradução de Cid Knipel Moreira. 1. ed. São Paulo: Saraiva, 2003. tradução da 9 ed americana.

SEVERINO, A. J. Metodologia do Trabalho Científico. 9. ed. São Paulo: Cortez, 1983.

SMITH, W. R. Product Differentiation and Market Segmentation as Alternative Marketing Strategies. The Journal of Marketing, v. 21, p. 3-8, Jul 1956.

SOUZA, L. A. D. Sistema de pagamentos brasileiro: nova estrutura e seus impactos econômicos. 1. ed. São Paulo: Saraiva, 2001.

WIND, Y. Issues and Advances in Segmentation Research. Journal of Marketing Research, v. 15, p. 317-337, Aug 1978. 


\section{APÊNDICE}

\section{Apêndice 1: Programação do PID}

if DESC_MACRORAMO = 'Alimentos' and PLANO $=1$ and VALOR $>=0$ and VALOR $<12$ then PID $=1$;else */Alimentos_1_F01:00|-12/* if DESC_MACRORAMO = 'Construção' and PLANO $=1$ and VALOR $>=0$ and VALOR $<15$ then PID $=2$;else */Construção 1 -F01:0|--15 if DESC_MACRORAMO = 'Diversão' and PLANO $=1$ and VALOR $>=0$ and VALOR $<12$ then PID $=3$; else */Diversão_1_F01:01--12/* if DESC_MACRORAMO = 'Farmácia' and PLANO $=1$ and VALOR $>=0$ and VALOR $<11$ then PID= 4 ;else */Farmácia_1_F01:0|--11 if DESC_MACRORAMO $=$ 'Geral' and PLANO $=1$ and VALOR $>=0$ and VALOR $<13$ then PID $=5$;else */Geral_1_FO1:0|--13/* if DESC_MACRORAMO $=$ 'Joalherias' and PLANO $=1$ and VALOR $>=0$ and VALOR $<12$ then PID $=6$;else */Joalherias_1_F01:0|--12/

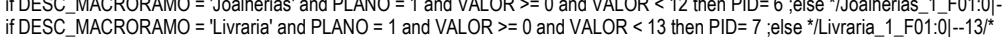
if DESC_MACRORAMO $=$ 'Lojas em Geral' and PLANO $=1$ and VALOR $>=0$ and VALOR $<13$ then PID $=8$; else */Lojas em Geral 1 F F01:0|--13/*

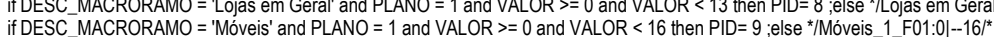

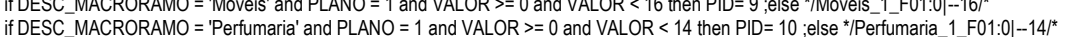
if DESC_MACRORAMO = 'Postos de Gasolina' and PIANO $=1$ and VALOR $>=0$ an $V A L O R<18$ then PID $=11$; else */Postos de Gasolina 1_F01:01--18/" if DESC_MACRORAMO $=$ 'Postos de Gasolina' and PLANO $=1$ and VALOR $>=0$ and VALOR $<18$ then PID $=11 ;$;else */Postos de Gasoln
if DESC_MACRORAMO $=$ 'Serviços' and PLANO $=1$ and VALOR $>=0$ and VALOR $<16$ then PID $=12 ;$;else */Serviços_1 F01:0|--16/*

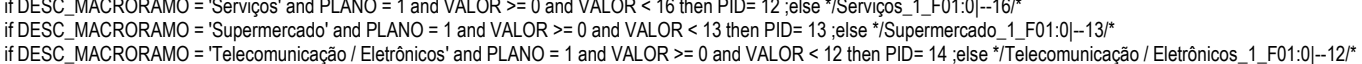
if DESC_MACRORAMO $=$ 'Telecomunicação / Eletrônicos' and PLANO $=1$ and VALOR $>=0$ and VALOR $<12$ then PID $=14 ;$;else */Telecomu
if DESC_MACRORAMO $=$ 'Vestuário' and PLANO $=1$ and VALOR $>=0$ and VALOR $<15$ then PID $=15 ;$;else */Vestuário_1_F01:0|-15/* if DESC_MACRORAMO = 'Veículos' and PLANO $=1$ and VALOR $>=0$ and VALOR $<16$ then PID $=16$;else * $/$ veiculos_1_FO1:0|--16/* if DESC_MACRORAMO $=$ 'Viagens' and PLANO $=1$ and VALOR $>=0$ and VALOR $<20$ then PID $=17$; else */Viagens_1_F01:0| $-\left.20\right|^{*}$ if DESC_MACRORAMO $=$ 'Alimentos' and PLANO $=2$ and VALOR $>=0$ and VALOR $<50$ then PID $=18 ;$;else */Alimentos_2_F01:0|--50/* if DESC_MACRORAMO = 'Construção' and PLANO $=2$ and VALOR $>=0$ and VALOR $<50$ then PID $=19$;else */Construçãa_2_F01:0|-50/* if DESC_MACRORAMO = 'Diversão' and PLANO $=2$ and VALOR $>=0$ and VALOR $<47$ then PID $=20$;else */Diversão_2_F01:00|-47/* if DESC_MACRORAMO $=$ 'Farmácia' and PLANO $=2$ and VALOR $>=0$ and VALOR $<27$ then PID $=21$;else */Farmácia_2_F01:0|--27/ if DESC_MACRORAMO = 'Geral' and PLANO $=2$ and VALOR $>=0$ and VALOR $<40$ then PID $=22$;else */Geral 2 F01:0|--40/*

if DESC_MACRORAMO = 'Joalherias' and PLANO $=2$ and VALOR $>=0$ and VALOR $<37$ then PID= 23 ;else */Joalherias 2 F01:0|-37/

if DESC_MACRORAMO = 'Livraria' and PLANO $=2$ and VALOR $>=0$ and VALOR $<38$ then PID= 24 ;else */Livraria 2 F01:0|--38/*

if DESC_MACRORAMO = 'Lojas em Geral' and PLANO $=2$ and VALOR $>=0$ and VALOR $<33$ then PID $=25$;else */Lojas em Geral 2 2 F01:0|-33/*

if DESC_MACRORAMO $=$ 'Móveis' and PLANO $=2$ and VALOR $>=0$ and VALOR $<43$ then PID $=26 ;$;else */Móveis_2_F01:00|--43/*

if DESC_MACRORAMO = 'Perfumaria' and PLANO $=2$ and VALOR $>=0$ and VALOR $<35$ then PID $=27$; else */Perfumaria_2_F01:0|-35/*

if DESC_MACRORAMO $=$ 'Postos de Gasolina' and PLANO $=2$ and VALOR $>=0$ and VALOR $<34$ then PID $=28$;else */Postos de Gasolina_2 F F 1:01--34/*

if DESC_MACRORAMO $=$ 'Serviços' and PLANO $=2$ and VALOR $>=0$ and VALOR $<45$ then PID $=29$;else */Serviços_2_F01:0|--45/*

if DESC_MACRORAMO $=$ 'ServiçOS' and PLANO $=2$ and VALOR $>=0$ and VALOR $<45$ then PID $=29 ;$;else */ServiçOS_2_F01:0|--45/*
if DESC_MACRORAMO $=$ 'Supermercado' and PLANO $=2$ and VALOR $>=0$ and VALOR $<20$ then PID $=30$;else $* /$ Supermercado 2 F01:01-20/*

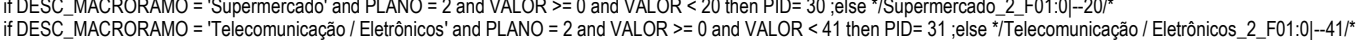

if DESC_MACRORAMO $=$ 'Telecomunicação / Eletrônicos' and PLANO $=2$ and VALOR $>=0$ and VALOR $<41$ then PID $=31 ;$;else * $/$ Telecom
if DESC_MACRORAMO $=$ 'Vestuário' and PLANO $=2$ and VALOR $>=0$ and VALOR $<35$ then PID $=32 ;$;else * $/$ Vestuário_2_F01:0|--35/*

if DESC_MACRORAMO $=$ 'Veiculos' and PLANO $=2$ and VALOR $>=0$ and VALOR $<50$ then PID $=33$;else * $*$ Veículos_2_F01:0|--50/

if DESC_MACRORAMO = Vlagens' and PLANO $=2$ and VALOR $>=0$ and VALOR $<71$ then PID $=34$, else "Niagens_2_F01:0|-- $-11 /{ }^{*}$

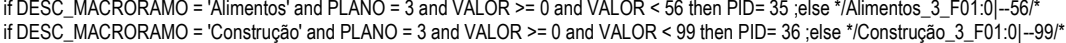

if DESC_MACRORAMO = 'Diversão' and PLANO $=3$ and VALOR $>=0$ and VALOR $<75$ then PID $=37$;else */Diversão_3_F01:0|--75/*

if DESC_MACRORAMO = 'Farmácia' and PLANO $=3$ and VALOR $>=0$ and VALOR $<41$ then PID $=38$;else */Farmácia_3_F01:0|--41/*

if DESC_MACRORAMO $=$ 'Geral' and PLANO $=3$ and VALOR $>=0$ and VALOR $<71$ then PID $=39 ;$;else $* /$ Geral_3_F01:0|--71/ ${ }^{*}$

if DESC_MACRORAMO $=$ 'Joalherias' and PLANO $=3$ and VALOR $>=0$ and VALOR $<68$ then PID $=40$; ;lse */Joalherias_3_F01:00|-68/"

if DESC_MACRORAMO = 'Livraria' and PLANO $=3$ and VALOR $>=0$ and VALOR $<63$ then PID $=41$;else */Livraria_3_F01:0|--63/*

if DESC_MACRORAMO = 'Lojas em Geral' and PLANO $=3$ and VALOR $>=0$ and VALOR $<55$ then PID $=42$; else */Lojas em Geral_3_F01:0|--55/*

if DESC_MACRORAMO = 'Móveis' and PLANO $=3$ and VALOR $>=0$ and VALOR $<86$ then PID $=43$;else */Móveis_ 3 F01:01--86/"

if DESC_MACRORAMO = 'Perfumaria' and PLANO $=3$ and VALOR $>=0$ and VALOR $<67$ then PID $=44$;else */Perfumaria_3_F01:0|--67/*

if DESC _MACRORAMO = 'Postos de Gasolina' and PLANO $=3$ and VALOR $>=0$ and VALOR $<40$ then PID $=45$;else */Postos de Gasolina 3 F01:0|--40/*

if DESC_MACRORAMO = 'Serviços' and PLANO $=3$ and VALOR $>=0$ and VALOR $<85$ then PID= 46 ;else */Serviços 3 F01:0|--85/*

if DESC_MACRORAMO = 'Supermercado' and PLANO $=3$ and VALOR $>=0$ and VALOR $<29$ then PID $=47$;else */Supermercado_3_F01:0|-29/*

if DESC MACRORAMO = 'Telecomunicação / Eletrônicos' and PLANO $=3$ and VALOR $>=0$ and VALOR $<79$ then PID $=48$.else */Telecomunicação/Eletrônicos 3 F01.0|--79/* if DESC_MACRORAMO = 'Vestuário' and PLANO $=3$ and VALOR $>=0$ and VALOR $<59$ then PID $=49$;else */Vestuário_3_F01:0|-59/* if DESC_MACRORAMO $=$ 'Veículos' and PLANO $=3$ and VALOR $>=0$ and VALOR $<95$ then PID $=50$;else * $/$ Veiculos_3_F01:0|--95/* if DESC_MACRORAMO = Veiculos' and PLANO $=3$ and VALOR $>=0$ and VALOR $<95$ then PID $=50$, else "Neiculos_3_F01:0 if DESC_MACRORAMO $=$ 'Viagens' and PLANO $=3$ and VALOR $>=0$ and VALOR $<125$ then PID $=51$;else */Viagens_3_F01:0 -1 - $1255^{*}$

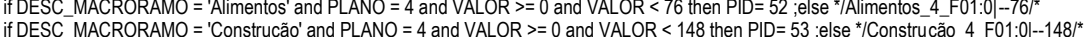

if DESC_MACRORAMO $=$ 'Diversão' and PLANO $=4$ and VALOR $>=0$ and VALOR $<96$ then PID $=54$;else */Diversão_4_F01:0|--96/*

if DESC_MACRORAMO $=$ 'Diversão' and PLANO $=4$ and VALOR $>=0$ and VALOR $<96$ then PID $=54$;else */Diversão_4_F01:00|-96/*
if DESC_MACRORAMO $=$ 'Farmácia' and PLANO $=4$ and VALOR $>=0$ and VALOR $<52$ then PID $=55$;else */Farmácia_4_F01:0|--52/ ${ }^{*}$

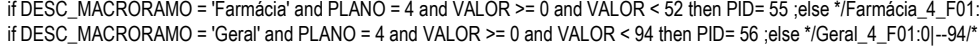

if DESC_MACRORAMO $=$ 'Geral' and PLANO $=4$ and VALOR $>=0$ and VALOR $<94$ then PID $=56$;else */Geral_4_FO1:0|--94/*
if DESC_MACRORAMO $=$ 'Joalherias' and PLANO $=4$ and VALOR $>=0$ and VALOR $<108$ then PID $=57 ;$;lse $/ /$ Joalherias_4_F01:0|-108/*

if DESC_MACRORAMO $=$ 'Joalherias' and PLANO $=4$ and VALOR $>=0$ and VALOR $<108$ then PID $=57$; else */Joalherias_4_F01:01-1
if DESC_MACRORAMO $=$ 'Livraria' and PLANO $=4$ and VALOR $>=0$ and VALOR $<87$ then PID $=58$;else */Livraria_4_F01:0|--87/ ${ }^{*}$

if DESC_MACRORAMO = 'Lojas em Geral' and PLANO $=4$ and VALOR $>=0$ and VALOR $<67$ then PID $=59$; else */Lojas em Geral_4_F01:0|- $67 /$ if DESC_MACRORAMO = 'Móveis' and PLANO $=4$ and VALOR $>=0$ and VALOR $<120$ then PID $=60$;else */Móveis_4_F01:0|-120/* if DESC_MACRORAMO = 'Perfumaria' and PLANO $=4$ and VALOR $>=0$ and VALOR $<99$ then PID $=61$; else */Perfumaria_4_F01:0|--99/* if DESC_MACRORAMO $=$ 'Postos de Gasolina' and PLANO $=4$ and VALOR $>=0$ and VALOR $<50$ then PID $=62$; else $* /$ Postos de Gasolina_4_F01:0|--50/" if DESC_MACRORAMO = 'Serviç̧os' and PLANO = 4 and VALOR $>=0$ and VALOR $<120$ then PID= 63 ;else */Serviç̧os_4_F01:0|-120/*

if DESC_MACRORAMO = 'Supermercado' and PLANO $=4$ and VALOR $>=0$ and VALOR $<39$ then PID= 64 ;else */Supermercado_4_F01:0|-39/*

if DESC MACRORAMO = 'Telecomunicação / Eletrônicos' and PLANO = 4 and VALOR $>=0$ and VALOR $<99$ then PID 65 ;else */Telecomunicação / Eletrônicos 4 _F01:0|--99/*

if DESC_MACRORAMO = 'Vestuário' and PLANO $=4$ and VALOR $>=0$ and VALOR $<80$ then PID = 66 ;else */Vestuário_4_F01:01--80/*

if DESC_MACRORAMO = 'Veículos' and PLANO $=4$ and VALOR $>=0$ and VALOR $<146$ then PID $=67$;else * Veiculos_4_F01:01--146/*

if DESC_MACRORAMO $=$ 'Viagens' and PLANO $=4$ and VALOR $>=0$ and VALOR $<217$ then PID $=68$;else */Viagens_4_F01:0| $-217 /^{*}$

if DESC_MACRORAMO = 'Alimentos' and PLANO $=5$ and VALOR $>=0$ and VALOR $<196$ then PID $=69$;else */Alimentos_5_F01:0|- $-196 /{ }^{*}$

if DESC_MACRORAMO = 'Construção' and PLANO $=5$ and VALOR $>=0$ and VALOR $<179$ then PID $=70$; else */Construção_5_F01:0|-179

if DESC_MACRORAMO = 'Diversão' and PLANO $=5$ and VALOR $>=0$ and VALOR $<131$ then PID $=71$;else */Diversão_5_F01:0|--131/*

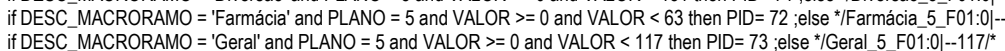

if DESC_MACRORAMO $=$ 'Geral' and PLANO $=5$ and VALOR $>=0$ and VALOR $<117$ then PID $=73$;else */Geral_5_FO1:0|--117/ $\left.\right|^{*}$
if DESC_MACRORAMO $=$ 'Joalherias' and PLANO $=5$ and VALOR $>=0$ and VALOR $<153$ then PID $=74$;else */Joalherias_5_F01:00|-153/*

if DESC_MACRORAMO $=$ 'Joalherias' and PLANO $=5$ and VALOR $>=0$ and VALOR $<153$ then PID $=74$;else */Joalherias_5_FO1:0|--
if DESC_MACRORAMO $=$ 'Livraria' and PLANO $=5$ and VALOR $>=0$ and VALOR $<110$ then PID $=75$;else $*$ Livraria_5_F01:0|--110/*

if DESC_MACRORAMO $=$ 'Livraria' and PLANO $=5$ and VALOR $>=0$ and VALOR $<110$ then PID $=75$;else */Livraria_5_F01:0|--110/*
if DESC_MACRORAMO $=$ 'Lojas em Geral' and PLANO $=5$ and VALOR $>=0$ and VALOR $<88$ then PID $=76$;else $* /$ Lojas em Geral_5_F01:0|--88/

if DESC_MACRORAMO $=$ 'Lojas em Geral' and PLANO $=5$ and VALOR $>=0$ and VALOR $<88$ then PID $=76$; else */Lojas em Geral_5_
if DESC_MACRORAMO $=$ 'Móveis' and PLANO $=5$ and VALOR $>=0$ and VALOR $<150$ then PID $=77 ;$;else */Móveis_5_F01:0|--150/*

if DESC_MACRORAMO $=$ 'Moveis' and PLANO $=5$ and VALOR $>=0$ and VALOR $<150$ then PID $=77 ;$;else */Móveis_5_F01:0|--150/*
if DESC_MACRORAMO $=$ 'Perfumaria' and PLANO $=5$ and VALOR $>=0$ and VALOR $<105$ then PID $=78$;else */Perfumaria_5_F01:0|-105/*

if DESC_MACRORAMO $=$ 'Perfumaria' and PLANO $=5$ and VALOR $>=0$ and VALOR $<105$ then PID $=78$;else */Perfumaria_5_F01:0|--105/*
if DESC_MACRORAMO $=$ 'Postos de Gasolina' and PLANO $=5$ and VALOR $>=0$ and VALOR $<30$ then PID $=79$; else $* /$ Postos de Gasolina_5_F01:01-30/

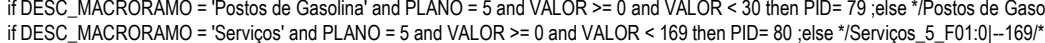

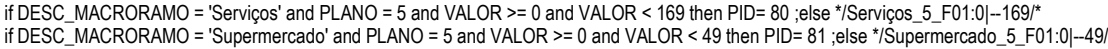

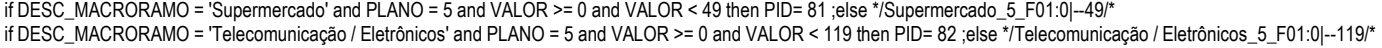
if DESC_MACRORAMO $=$ 'Vestuário' and PLANO $=5$ and VALOR $>=0$ and VALOR $<94$ then PID $=83$;else */Vestuário_5_F01:0| $-94{ }^{*}$

if DESC_MACRORAMO $=$ 'Veículos' and PLANO $=5$ and VALOR $>=0$ and VALOR $<193$ then PID $=84 ;$;else * *Veiculos_5 F01:0|--193/

if DESC MACRORAMO $=$ 'Viagens' and PLANO $=5$ and VALOR $>=0$ and VALOR $<248$ then PID $=85$; else */Viagens 5 F $01: 0 \mid-248 /{ }^{*}$

if DESC_MACRORAMO = 'Alimentos' and PLANO $=6$ and VALOR $>=0$ and VALOR $<89$ then PID= 86 ;else */Alimentos_6_F01:0|--89/*

if DESC_MACRORAMO = 'Construção' and PLANO $=6$ and VALOR $>=0$ and VALOR $<236$ then PID= 87 ;else */Construção_6_F01:0|-236/*

if DESC_MACRORAMO = 'Diversão' and PLANO $=6$ and VALOR $>=0$ and VALOR $<290$ then PID $=88$;else */Diversão_6_F01:0|--290/*

if DESC_MACRORAMO $=$ 'Farmácia' and PLANO $=6$ and VALOR $>=0$ and VALOR $<42$ then PID $=89$;else */Farmácia_ 6 F01:0|--42

if DESC MACRORAMO = 'Joalherias' and PLANO $=6$ and VALOR $>=0$ and VALOR $<200$ then PID $=91$; else */Joalherias 6 F01:0|--200/* 
if DESC_MACRORAMO $=$ 'Livraria' and PLANO $=6$ and VALOR $>=0$ and VALOR $<137$ then PID $=92$;else */Livraria_6_F01:0|- $-137 /{ }^{*}$ if DESC_MACRORAMO = 'Lojas em Geral' and PLANO $=6$ and VALOR $>=0$ and VALOR $<105$ then PID $=93$;else */Lojas em Geral_6_F01:0|- $105 /{ }^{*}$ if DESC_MACRORAMO = 'Móveis' and PLANO $=6$ and VALOR $>=0$ and VALOR $<208$ then PID $=94$;else */Móveis_6_F01:0|--208/ ${ }^{*}$ if DESC MACRORAMO = 'Perfumaria' and PLANO $=6$ and VALOR $>=0$ and VALOR $<140$ then PID $=95$.else */Perfumaria 6 F01:01--140/* if DESC_MACRORAMO = 'Postos de Gasolina' and PLANO $=6$ and VALOR $>=0$ and VALOR $<317$ then PID $=96$; else */Postos de Gasolina_6_F01:0|--317/*

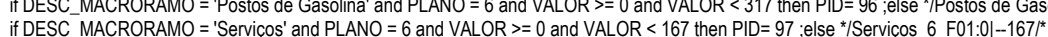
if DESC_MACRORAMO $=$ 'ServiçOS' and PLANO $=6$ and VALOR $>=0$ and VALOR $<167$ then PID $=97$;else */Serviços_6_F01:0|-167/*
if DESC_MACRORAMO $=$ 'Supermercado' and PLANO $=6$ and VALOR $>=0$ and VALOR $<43$ then PID $=98$;else */Supermercado 6 F01:0|--43/*

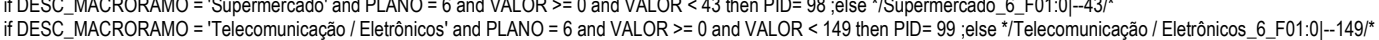

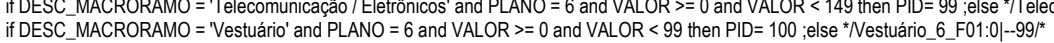

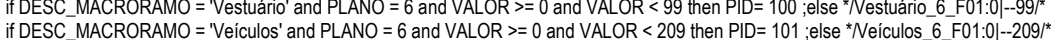

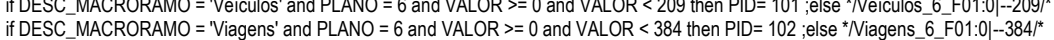
if DESC_MACRORAMO $=$ 'Viagens' and PLANO $=6$ and VALOR $>=0$ and VALOR $<384$ then PID $=102$;else *Viagens_6_F01:0|--384/*
if DESC_MACRORAMO $=$ 'Construção' and PLANO $=7$ and VALOR $>=0$ and VALOR $<250$ then PID $=103$; else */Construçẫo 7 F01:00|-250/* if DESC_MACRORAMO = 'Diversão' and PLANO $=7$ and VALOR $>=0$ and VALOR $<300$ then PID $=104$;else */Diversão_7_F01:0|-300/ if DESC_MACRORAMO = 'Farmácia' and PLANO $=7$ and VALOR $>=0$ and VALOR $<74$ then PID $=105$; else */Farmácia_7_F01:0|--74/* if DESC_MACRORAMO = 'Geral' and PLANO $=7$ and VALOR $>=0$ and VALOR $<79$ then PID $=106$;else */Geral_7_F01:0|--79/ if DESC_MACRORAMO $=$ 'Joalherias' and PLANO $=7$ and VALOR $>=0$ and VALOR $<220$ then PID $=107$; else */Joalherias_7_F01:0|--220/ if DESC_MACRORAMO = 'Livraria' and PLANO $=7$ and VALOR $>=0$ and VALOR $<156$ then PID $=108$;else */Livraria_7_F01:0|--156/* if DESC_MACRORAMO $=$ 'Lojas em Geral' and PLANO $=7$ and VALOR $>=0$ and VALOR $<97$ then PID $=109$;else */Lojas em Geral_7_F01:0|--97/ ${ }^{*}$ if DESC_MACRORAMO = 'Móveis' and PLANO $=7$ and VALOR $>=0$ and VALOR $<219$ then PID= 110 ;else */Móveis_7_F01:0|--219/* if DESC_MACRORAMO = 'Perfumaria' and PLANO $=7$ and VALOR $>=0$ and VALOR $<100$ then PID $=111$; else */Perfumaria_7_F01:0|--100/* if DESC_MACRORAMO = 'Serviços' and PLANO $=7$ and VALOR $>=0$ and VALOR $<79$ then PID $=112$; else */Servicos 7 F $01: 0 \mid-79 /^{*}$ if DESC_MACRORAMO $=$ 'Supermercado' and PLANO $=7$ and VALOR $>=0$ and VALOR $<70$ then PID $=113$;else */Supermercado_7_F01:00|-70/*

if DESC_MACRORAMO = 'Telecomunicação / Eletrônicos' and PLANO $=7$ and VALOR $>=0$ and VALOR $<183$ then PID $=114$;else */Telecomunicação / Eletrônicos_7_F01:0|--183/* if DESC_MACRORAMO $=$ 'Vestuário' and PLANO $=7$ and VALOR $>=0$ and VALOR $<97$ then PID $=115$; ; lse * $*$ Vestuário_7_FO1:0|--97/* if DESC_MACRORAMO $=$ 'Veículos' and PLANO $=7$ and VALOR $>=0$ and VALOR $<157$ then PID $=116$; ;lse * $*$ eiculos_7 7 F01:0|--157/ if DESC_MACRORAMO $=$ 'Construção' and PLANO $=8$ and VALOR $>=0$ and VALOR $<359$ then PID $=118$; else */Construção_ 8 F F01:0|--359/* if DESC_MACRORAMO $=$ 'Construção' and PLANO $=8$ and VALOR $>=0$ and VALOR $<359$ then PID $=118$;else */Construção_8_F01:0|--359*
if DESC_MACRORAMO $=$ 'Diversão' and PLANO $=8$ and VALOR $>=0$ and VALOR $<560$ then PID $=119 ;$;else */Diversão_8_F01:0|--560/* if DESC_MACRORAMO $=$ 'Diversão' and PLANO $=8$ and VALOR $>=0$ and VALOR $<560$ then PID $=119$;else */Diversão_8_F01:0|--560/*
if DESC_MACRORAMO $=$ 'Farmácia' and PLANO $=8$ and VALOR $>=0$ and VALOR $<81$ then PID $=120$;else */Farmácia 8 F01:0|--81/

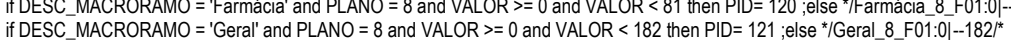
if DESC_MACRORAMO $=$ 'Geral' and PLANO $=8$ and VALOR $>=0$ and VALOR $<182$ then PID $=121$;else */Geral_8_F01:0|- $-182 /^{*}$
if DESC_MACRORAMO $=$ 'Joalherias' and PLANO $=8$ and VALOR $>=0$ and VALOR $<345$ then PID $=122$ else */Joalherias_8 8 F01:01--345/*

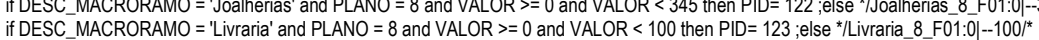
if DESC_MACRORAMO = 'Lojas em Geral' and PLANO $=8$ and VALOR $>=0$ and VALOR $<123$ then PID $=124$; ;else */Lojas em Geral_8_F01:0|--123/ if DESC_MACRORAMO = 'Móveis' and PLANO $=8$ and VALOR $>=0$ and VALOR $<256$ then PID= 125 ;else */Móveis_8_F01:0|--256/ if DESC_MACRORAMO = 'Perfumaria' and PLANO $=8$ and VALOR $>=0$ and VALOR $<218$ then PID $=126$;else */Perfumaria_8_F01:0|-218/* if DESC_MACRORAMO = 'Serviços' and PLANO $=8$ and VALOR $>=0$ and VALOR $<220$ then PID $=127$; else */Serviços_8_F01:0|-220/* if DESC_MACRORAMO = 'Supermercado' and PLANO $=8$ and VALOR $>=0$ and VALOR $<89$ then PID $=128$;else */Supermercado_8_F01:0|--89/* if DESC_MACRORAMO = 'Telecomunicação / Eletrônicos' and PLANO $=8$ and VALOR $>=0$ and VALOR $<209$ then PID $=129$;else */Telecomunicação / Eletrônicos_8_F01:0|--209/* if DESC_MACRORAMO = 'Vestuário' and PLANO $=8$ and VALOR $>=0$ and VALOR $<115$ then PID $=130$;else */Nestuário_8_F01:0|--115/* if DESC_MACRORAMO = 'Veículos' and PLANO $=8$ and VALOR $>=0$ and VALOR $<264$ then PID= 131 ;else * $/$ eículos_8_F01:0|--264/" if DESC_MACRORAMO = 'Viagens' and PLANO $=8$ and VALOR $>=0$ and VALOR $<408$ then PID $=132$;else * Viagens 8 F01:0|--408/* if DESC_MACRORAMO = 'Construçãa' and PLANO $=9$ and VALOR $>=0$ and VALOR $<416$ then PID $=133$; ;else */Construção_9_F01:0|- $416 /{ }^{*}$ if DESC_MACRORAMO = 'Diversão' and PLANO $=9$ and VALOR $>=0$ and VALOR $<337$ then PID $=134$;else */Diversão 9 F01:0|--337/* if DESC MACRORAMO = 'Farmácia' and PLANO $=9$ and VALOR $>=0$ and VALOR $<92$ then PID $=135$; else */Farmácia 9 F01:0|--92/* if DESC_MACRORAMO = 'Geral' and PLANO $=9$ and VALOR $>=0$ and VALOR $<94$ then PID $=136$;else */Geral_9_F01:0|--94/* if DESC_MACRORAMO $=$ 'Joalherias' and PLANO $=9$ and VALOR $>=0$ and VALOR $<98$ then PID $=137$; else */Joalherias_9_F01:0|--98/* if DESC_MACRORAMO = 'Livraria' and PLANO $=9$ and VALOR $>=0$ and VALOR $<97$ then PID $=138$; ; else */Livraria_9_F01:0|--97/* if DESC_MACRORAMO = 'Lojas em Geral' and PLANO $=9$ and VALOR $>=0$ and VALOR $<99$ then PID $=139$;else */Lojas em Geral_9_F01:0|--99/* if DESC_MACRORAMO = 'Móveis' and PLANO $=9$ and VALOR $>=0$ and VALOR $<376$ then PID $=140$; else */Móveis 99 F01:0|--376/* if DESC_MACRORAMO $=$ 'Móveis' and PLANO $=9$ and VALOR $>=0$ and VALOR $<376$ then PID $=140$;else */Móveis_9_F01:0|--376/*
if DESC MACRORAMO $=$ 'Serviços' and PLANO $=9$ and VALOR $>=0$ and VALOR $<132$ then PID $=141$; else */Serviços 9 F01:0|-132/*

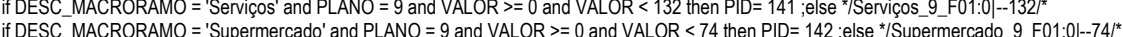

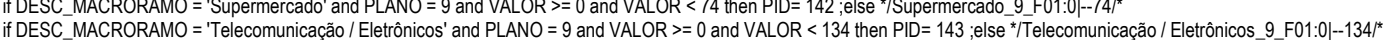

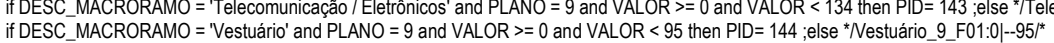
if DESC_MACRORAMO = 'Vestuario' and PLANO $=9$ and VALOR $>=0$ and VALOR $<95$ then PID $=144$; else * Nestuario_9_F01:0|--95/* if DESC_MACRORAMO $=$ 'Veículos' and PLANO $=9$ and VALOR $>=0$ and VALOR $<480$ then PID $=145$;else *Veículos_9_F01:0|--480/*
if DESC_MACRORAMO $=$ 'Viagens' and PLANO $=9$ and VALOR $>=0$ and VALOR $<466$ then PID $=146$;else *Niagens_ 9 F01:0|--466/*

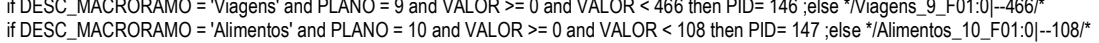
if DESC_MACRORAMO = 'Construção' and PLANO $=10$ and VALOR $>=0$ and VALOR $<350$ then $\mathrm{PID}=148$;else */Construção_10_F01:0|--350/* if DESC_MACRORAMO = 'Diversão' and PLANO $=10$ and VALOR $>=0$ and VALOR $<234$ then PID= 149 ;else */Diversão_10_F01:0|-234/" if DESC_MACRORAMO $=$ 'Farmácia' and PLANO $=10$ and VALOR $>=0$ and VALOR $<63$ then PID $=150$;else */Farmácia_10_F01:0|--63/" if DESC_MACRORAMO = 'Geral' and PLANO $=10$ and VALOR $>=0$ and VALOR $<229$ then PID $=151$; else */Geral 10 F01:0| $-229 /{ }^{*}$ if DESC_MACRORAMO $=$ 'Joalherias' and PLANO $=10$ and VALOR $>=0$ and VALOR $<300$ then $P I D=152$; else $* /$ Joalherias_10_F01:0|--300/* if DESC_MACRORAMO = 'Livraria' and PLANO $=10$ and VALOR $>=0$ and VALOR $<126$ then PID $=153$; else */Livraria_10_F01:0|-126/* if DESC_MACRORAMO = 'Lojas em Geral' and PLANO $=10$ and VALOR $>=0$ and VALOR $<149$ then PID $=154$;else */Lojas em Geral_10 F01:0|--149/ if DESC_MACRORAMO = 'Móveis' and PLANO $=10$ and VALOR $>=0$ and VALOR $<299$ then PID $=155$;else */Móveis 10 F01:0|-299/* if DESC_MACRORAMO $=$ 'Perfumaria' and PLANO $=10$ and VALOR $>=0$ and VALOR $<148$ then PID $=156$;else */Perfumaria_10 F01:0|-148 ${ }^{*}$ if DESC_MACRORAMO $=$ 'Postos de Gasolina' and PLANO $=10$ and VALOR $>=0$ and VALOR $<303$ then PID $=157$;else */Postos de Gasolina 10 F01:0|-303/* if DESC_MACRORAMO = 'Serviços' and PLANO $=10$ and VALOR $>=0$ and VALOR $<249$ then PID $=158$;else */Serviços_10_F01:0|--249/* if DESC_MACRORAMO = 'Supermercado' and PLANO $=10$ and VALOR $>=0$ and VALOR $<99$ then PID $=159$; else */Supermercado 10 F01:0|--99/* if DESC_MACRORAMO $=$ 'Telecomunicação/ Eletrônicos' and PLANO $=10$ and VALOR $>=0$ and VALOR $<199$ then PID $=160$;else */Telecomunicação/Eletrônicos_10_F01:01--199/* if DESC_MACRORAMO = 'Vestuário' and PLANO $=10$ and VALOR $>=0$ and VALOR $<134$ then PID $=161$; else * $/$ estuário_10_F01:0|--134/ ${ }^{*}$

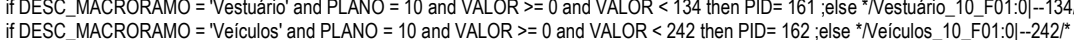
if DESC_MACRORAMO $=$ 'Veiculos' and PLANO $=10$ and VALOR $>=0$ and VALOR $<242$ then PID $=162$;else */Veiculos_10_F01:0| $\mid-242{ }^{*}$
if DESC_MACRORAMO $=$ 'Viagens' and PLANO $=10$ and VALOR $>=0$ and VALOR $<297$ then PID $=163$;else * $/$ Viagens_10_F01:0|-297/* if DESC_MACRORAMO $=$ 'Viagens' and PLANO $=10$ and VALOR $>=0$ and VALOR $<297$ then PID $=163$;else *Niagens_10_FO1:0|--297
if DESC_MACRORAMO $=$ 'Geral' and PLANO $=11$ and VALOR $>=0$ and VALOR $<220$ then PID $=164$;else */Geral_11_F01:0|-220/* if DESC_MACRORAMO = 'Geral' and PLANO $=11$ and VALOR $>=0$ and VALOR $<220$ then PID $=164$;else */Geral_11_F01:0|-220/* if DESC_MACRORAMO $=$ 'Lojas em Geral' and PLANO $=11$ and VALOR $>=0$ and VALOR $<341$ then PID $=166$; ; else ${ }^{*} /$ Lojas em Geral_11_F01:00|-341/ if DESC_MACRORAMO = 'Móveis' and PLANO $=11$ and VALOR $>=0$ and VALOR $<300$ then PID $=167$;else */Móveis_11_F01:0|--300/* if DESC_MACRORAMO = 'Supermercado' and PLANO = 11 and VALOR $>=0$ and VALOR $<99$ then PID $=168$;else */Supermercado_11_F01:01--99/* if DESC_MACRORAMO = 'Telecomunicação / Eletrônicos' and PLANO = 11 and VALOR $>=0$ and VALOR $<588$ then PID = 169 ;else */Telecomunicação / Eletrônicos_11_F01:0|--588/ ${ }^{*}$ if DESC_MACRORAMO = 'Vestuário' and PLANO $=11$ and VALOR $>=0$ and VALOR $<166$ then PID= 170 ;else *Nestuário_11_F01:00|-166/ if DESC_MACRORAMO = 'Construção' and PLANO $=12$ and VALOR $>=0$ and VALOR $<307$ then PID $=171$; else */Construção_12_F01:00|--307/* if DESC_MACRORAMO = 'Farmácia' and PLANO $=12$ and VALOR $>=0$ and VALOR $<114$ then PID = 172 ;else */Farmácia_12_F01:0|-114/* if DESC MACRORAMO = 'Geral' and PLANO $=12$ and VALOR $>=0$ and VALOR $<327$ then PID $=173$;else */Geral 12_F01:0|--327/* if DESC_MACRORAMO = 'Joalherias' and PLANO $=12$ and VALOR $>=0$ and VALOR $<300$ then PID $=174$;else */Joalherias_12_F01:0|--300/* if DESC_MACRORAMO = 'Livraria' and PLANO $=12$ and VALOR $>=0$ and VALOR $<132$ then PID= 175 ;else */Livraria_12_F01:0|--132/* if DESC_MACRORAMO = 'Lojas em Geral' and PLANO $=12$ and VALOR $>=0$ and VALOR $<215$ then PID= 176 ; else */Lojas em Geral_12_F01:0|-215/* if DESC_MACRORAMO = 'Móveis' and PLANO $=12$ and VALOR $>=0$ and VALOR $<369$ then PID = 177 ;else */Móveis 12 F01:0|--369/* if DESC_MACRORAMO = 'Perfumaria' and PLANO $=12$ and VALOR $>=0$ and VALOR $<95$ then PID $=178$;else */Perfumaria 12 F01:00|--95 if DESC MACRORAMO = 'Serviços' and PLANO = 12 and VALOR $>=0$ and VALOR $<205$ then PID= 179 ;else */Servicos 12 F01:01.-205/* if DESC_MACRORAMO = 'Supermercado' and PLANO $=12$ and VALOR $>=0$ and VALOR $<168$ then PID $=180$;else */Supermercado_12_F01:0|--168/* if DESC_MACRORAMO = 'Telecomunicação / Eletrônicos' and PLANO $=12$ and VALOR $>=0$ and VALOR $<319$ then PID $=181$; else */Telecomunicação/ Eletrônicos_12 F01:0|--319/* if DESC_MACRORAMO = 'Vestuário' and PLANO $=12$ and VALOR $>=0$ and VALOR $<189$ then PID $=182$; else * Nestuário_12_F01:0|--189/*

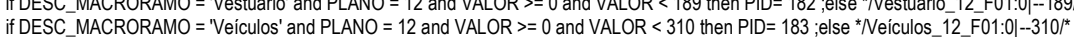

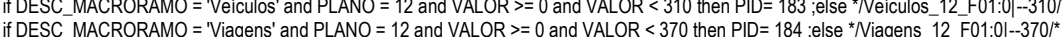
if DESC_MACRORAMO $=$ 'Viagens' and PLANO $=12$ and VALOR $>=0$ and VALOR $<370$ then PID $=184 ;$;else * $/$ Viagens_12_F01:0|--370/*
if DESC MACRORAMO $=$ 'Supermercado' and PLANO $=13$ and VALOR $>=0$ and VALOR $<598$ then PID $=185 ;$;else */Supermercado_13_F01:0|--598 ${ }^{*}$ if DESC_MACRORAMO $=$ 'Supermercado' and PLANO $=13$ and VALOR $>=0$ and VALOR $<598$ then PID $=185$;else */Supermercado_13_F01:0|--598/ ${ }^{*}$
if DESC_MACRORAMO $=$ 'Lojas em Geral' and PLANO $=14$ and VALOR $>=0$ and VALOR $<577$ then PID $=186$;else */Lojas em Geral_14_F01:0|--577/ if DESC_MACRORAMO $=$ 'Lojas em Geral' and PLANO $=14$ and VALOR $>=0$ and VALOR $<577$ then PID $=186$; else */Lojas em Geral_14_F01:0|--577/
if DESC_MACRORAMO $=$ 'Supermercado' and PLANO $=14$ and VALOR $>=0$ and VALOR $<40$ then PID $=187$ else */Supermercado 14 F01:0|--40/* if DESC_MACRORAMO $=$ 'Supermercado' and PLANO $=14$ and VALOR $>=0$ and VALOR $<40$ then PID $=187$;else */Supermercado_14_F01:0|--40/"
if DESC_MACRORAMO $=$ 'Construção' and PLANO $=15$ and VALOR $>=0$ and VALOR $<228$ then PID $=188$ else */Construcão 15 F01:0|--228/* if DESC_MACRORAMO $=$ 'Construção' and PLANO $=15$ and VALOR $>=0$ and VALOR $<228$ then PID $=188$; else */Construção_15_F01:01-22
if DESC_MACRORAMO = 'Livraria' and PLANO $=15$ and VALOR $>=0$ and VALOR $<910$ then PID $=189$ else */Livraria_15_F01:0|--910/* if DESC_MACRORAMO $=$ 'Livraria' and PLANO $=15$ and VALOR $>=0$ and VALOR $<910$ then PID $=189$;else */Livraria_15_F01:0|--910/*
if DESC_MACRORAMO $=$ 'Lojas em Geral' and PLANO $=15$ and VALOR $>=0$ and VALOR $<159$ then PID $=190 ;$;else $* /$ Lojas em Geral_15_F01:00|-159/" if DESC_MACRORAMO = 'Supermercado' and PLANO $=15$ and VALOR $>=0$ and VALOR $<248$ then PID $=191$;else */Supermercado_15_F01:0|--248/* if DESC_MACRORAMO = 'Telecomunicação / Eletrônicos' and PLANO = 15 and VALOR >= 0 and VALOR < 1415 then PID = 192 ;else */Telecomunicação/Eletrônicos_15_F01:0|--1415/* 
if DESC_MACRORAMO = 'Supermercado' and PLANO $=18$ and VALOR $>=0$ and VALOR $<388$ then PID $=193$;else */Supermercado_18_F01:0|--388/ ${ }^{*}$ if DESC MACRORAMO $=$ 'Supermercado' and PLANO $=24$ and VALOR $>=0$ and VALOR $<158$ then $\mathrm{PID}=194$ "else */Supermercado 24 F01:01- 158 " if DESC_MACRORAMO = 'Alimentos' and PLANO $=1$ and VALOR $>=12$ and VALOR $<21$ then PID $=195$; else */Alimentos_1_F02:12|-21/* if DESC_MACRORAMO = 'Construção' and PLANO $=1$ and VALOR $>=15$ and VALOR $<26$ then PID= 196 ;else */Construção_1_F02:15|-26/* if DESC_MACRORAMO $=$ 'Diversão' and PLANO $=1$ and VALOR $>=12$ and VALOR $<19$ then PID $=197$; else */Diversão_1_FO2:12|-19/*

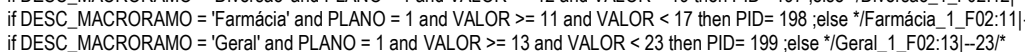
if DESC_MACRORAMO = 'Geral' and PLANO $=1$ and VALOR $>=13$ and VALOR $<23$ then PID $=199 ;$;else */Geral_1_FO2:13|--23/*
if DESC_MACRORAMO = 'Joalherias' and PLANO $=1$ and VALOR $>=12$ and VALOR $<19$ then PID 200 ;else */Joalherias 1 FO2:12|-19/* if DESC_MACRORAMO $=$ 'Joalherias' and PLANO $=1$ and VALOR $>=12$ and VALOR $<19$ then PID $=200$;else */Joalherias_1_F02:12|- $19{ }^{*}$ if DESC_MACRORAMO $=$ 'Livraria' and PLANO $=1$ and VALOR $>=13$ and VALOR $<22$ then PID $=201$;else */Livraria_1_F02:13|-22/* if DESC_MACRORAMO $=$ 'Livraria' and PLANO $=1$ and VALOR $>=13$ and VALOR $<22$ then PID $=201$;else */Livraria_1_FO2:13|-22/*
if DESC_MACRORAMO $=$ 'Lojas em Geral' and PLANO $=1$ and VALOR $>=13$ and VALOR $<21$ then PID $=202$;else $* /$ Lojas em Geral_1_F02:13|--21/* if DESC_MACRORAMO $=$ 'Lojas em Geral' and PLANO $=1$ and VALOR $>=13$ and VALOR $<21$ then PID $=202$;else */Lojas em Geral 1 -
if DESC MACRORAMO $=$ 'Móveis' and PLANO $=1$ and VALOR $>=16$ and VALOR $<27$ then PID $=203$;else */Móveis 1 F02:16|--27/*

if DESC_MACRORAMO $=$ 'Móveis' and PLANO $=1$ and VALOR $>=16$ and VALOR $<27$ then PID $=203 ;$;else */Móveis_1_FO2:16|--27/*
if DESC_MACRORAMO $=$ 'Perfumaria' and PLANO $=1$ and VALOR $>=14$ and VALOR $<21$ then PID $204:$ else */Perfumaria_1_F02:14|--21/*

if DESC_MACRORAMO $=$ 'Perfumaria' and PLANO $=1$ and VALOR $>=14$ and VALOR $<21$ then PID $=204$;else */Perfumaria_1_F02:14|--21/ ${ }^{*}$
if DESC_MACRORAMO $=$ 'Postos de Gasolina' and PLANO $=1$ and VALOR $>=18$ and VALOR $<30$ then PID $=205$;else */Postos de Gasolina_1_F02:18|-30/* if DESC_MACRORAMO = 'Serviços' and PLANO $=1$ and VALOR $>=16$ and VALOR $<30$ then PID $=206$;else */Serviços_1_F02:16|--30/* if DESC_MACRORAMO = 'Supermercado' and PLANO = 1 and VALOR $>=13$ and VALOR $<28$ then PID= 207 ;else */Supermercado_1_F02:13|-28/" if DESC_MACRORAMO = 'Telecomunicação / Elettrônicos' and PLANO $=1$ and VALOR $>=12$ and VALOR $<20$ then PID $=208$; ;lse ${ }^{*} /$ Telecomunicação / Eletrônicos_1_F02:12|--20/* if DESC_MACRORAMO = 'Vestuário' and PLANO $=1$ and VALOR $>=15$ and VALOR $<24$ then PID= 209 ;else *Vestuário_1_FO2:15|--24/* if DESC_MACRORAMO $=$ 'Veículos' and PLANO $=1$ and VALOR $>=16$ and VALOR $<27$ then PID $=210$;else *Neículos_ 1 - F02:16|-27/ if DESC_MACRORAMO = 'Viagens' and PLANO $=1$ and VALOR $>=20$ and VALOR $<32$ then PID $=211$;else */Viagens_1_F02:20|--32/ if DESC_MACRORAMO = 'Alimentos' and PLANO $=2$ and VALOR $>=50$ and VALOR $<75$ then PID= 212 ; else */Alimentos_2_F02:50|--75/* if DESC_MACRORAMO = 'Construção' and PLANO $=2$ and VALOR $>=50$ and VALOR $<74$ then PID= 213 ;else */Construção_2_F02:50|-74/ $\left.\right|^{*}$ if DESC_MACRORAMO = 'Diversão' and PLANO = 2 and VALOR >= 47 and VALOR $<64$ then PID $=214$;else */Diversão_2_F02:47|--64/* if DESC_MACRORAMO = 'Farmácia' and PLANO $=2$ and VALOR $>=27$ and VALOR $<38$ then PID $=215$;else */Farmácia 2 2F02:27|--38/* if DESC_MACRORAMO = 'Geral' and PLANO $=2$ and VALOR $>=40$ and VALOR $<56$ then PID= 216 ;else */Geral_2_F02:40|-56/* if DESC_MACRORAMO = 'Joalherias' and PLANO $=2$ and VALOR $>=37$ and VALOR $<50$ then PID $=217$; else */Joalherias_2_F02:37|-50/* if DESC_MACRORAMO $=$ 'Livraria' and PLANO $=2$ and VALOR $>=38$ and VALOR $<51$ then PID $=218$; else */Livraria_2_F02:38|-51/* if DESC_MACRORAMO $=$ 'Livraria' and PLANO $=2$ and VALOR $>=38$ and VALOR $<51$ then PID $=218$;else */Livraria_2_FO2:38|-51/*
if DESC_MACRORAMO $=$ 'Lojas em Geral' and PLANO $=2$ and VALOR $>=33$ and VALOR $<49$ then PID $=219 ;$;else */Lojas em Geral_2_F02:33|--49/*

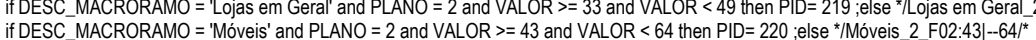
if DESC_MACRORAMO $=$ 'Móveis' and PLANO $=2$ and VALOR $>=43$ and VALOR $<64$ then PID $=220$;else */Móveis_2_F02:43|--64/*
if DESC_MACRORAMO $=$ 'Perfumaria' and PLANO $=2$ and VALOR $>=35$ and VALOR $<49$ then PID $=221$;else */Perfumaria_2_F02:35|--49/* if DESC_MACRORAMO $=$ 'Postos de Gasolina' and PLANO $=2$ and VALOR $>=34$ and VALOR $<50$ then PID $=222$; else */Postos de Gasolina 2 F02:34|--50/* if DESC_MACRORAMO = 'Postos de Gasolina' and PLANO $=2$ and VALOR $>=34$ and VALOR $<50$ then $P I D=222$;else */Postos de Gas if DESC_MACRORAMO $=$ 'Serviços' and PLANO $=2$ and VALOR $>=45$ and VALOR $<67$ then PID $=223$;else */Serviços_2_FO2:45|--67/*
if DESC_MACRORAMO = 'Supermercado' and PLANO $=2$ and VALOR $>=20$ and VALOR $<35$ then PID $=224$ :else */Supermercado 2 2 F02:20|--35/*

if DESC_MACRORAMO $=$ 'Supermercado' and PLANO $=2$ and VALOR $>=20$ and VALOR $<35$ then PID $=224$;else */Supermercado_2_F02:20|--35/*
if DESC_MACRORAMO $=$ 'Telecomunicaçãa / Eletrônicos' and PLANO $=2$ and VALOR $>=41$ and VALOR $<60$ then PID $=225$;else */Telecomunicação / Eletrônicos_2_F02:41|--60/* if DESC_MACRORAMO = 'Vestuário' and PLANO $=2$ and VALOR $>=35$ and VALOR $<49$ then PID $=226 ;$;else * $/$ Vestuário_2_F02:35|--49/* if DESC_MACRORAMO $=$ 'Veículos' and PLANO $=2$ and VALOR $>=50$ and VALOR $<73$ then PID $=227$;else * Neículos_2_F02:50|--73/* if DESC_MACRORAMO = 'Viagens' and PLANO $=2$ and VALOR $>=71$ and VALOR $<112$ then PID $=228$;else * $*$ Viagens_2_F02:71|--112/* if DESC_MACRORAMO = 'Alimentos' and PLANO $=3$ and VALOR $>=56$ and VALOR $<96$ then PID $=229$;else */Alimentos_3_F02:56|--96/* if DESC_MACRORAMO = 'Construção' and PLANO = 3 and VALOR $>=99$ and VALOR $<150$ then PID= 230 ;else */Construçãa_3_F02:99|--150/ if DESC_MACRORAMO = 'Diversão' and PLANO = 3 and VALOR >= 75 and VALOR < 109 then PID= 231 ;else */Diversão_3_F02:75|--109/* if DESC_MACRORAMO = 'Farmácia' and PLANO $=3$ and VALOR $>=41$ and VALOR $<60$ then PID= 232 ;else */Farmácia_3_F02:41|--60/* if DESC_MACRORAMO = 'Geral' and PLANO $=3$ and VALOR $>=71$ and VALOR $<110$ then PID $=233$;else */Geral_3_F02:71|--110/* if DESC_MACRORAMO = 'Joalherias' and PLANO $=3$ and VALOR >= 68 and VALOR $<99$ then PID= 234 ;else */Joalherias_3_F02:68|--99/* if DESC_MACRORAMO = 'Livraria' and PLANO $=3$ and VALOR $>=63$ and VALOR $<90$ then PID= 235 ;else */Livraria_3_F02:63|--90/*

if DESC_MACRORAMO = 'Lojas em Geral' and PLANO $=3$ and VALOR $>=55$ and VALOR $<82$ then PID $=236$;else */Lojas em Geral_ 3 F02:55|--82/* if DESC_MACRORAMO $=$ 'Móveis' and PLANO $=3$ and VALOR $>=86$ and VALOR $<149$ then PID= 237 ; ;lse */Móveis_3_F02:86|--149/*

if DESC_MACRORAMO = 'Perfumaria' and PLANO $=3$ and VALOR $>=67$ and VALOR $<9$ then PID $=238$;else */Perfumaria 3 F02:67|--89/* if DESC MACRORAMO $=$ 'Postos de Gasolina' and PLANO $=3$ and VALOR $>=40$ and VALOR $<60$ then PID $=239$ else */Postos de Gasolina 3 F02:40|--60/* if DESC_MACRORAMO = 'Serviços' and PLANO $=3$ and VALOR $>=85$ and VALOR $<137$ then PID $=240$;else */Serviços_3_FO2:85|--137/ ${ }^{*}$

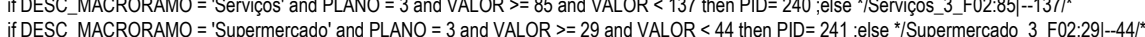

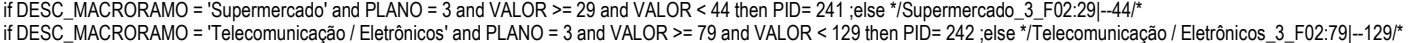

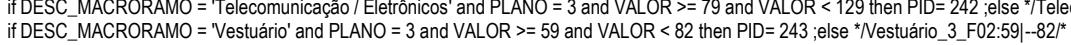
if DESC_MACRORAMO $=$ 'Vestuário' and PLANO $=3$ and VALOR $>=59$ and VALOR $<82$ then PID $=243$;else * $*$ Nestuário_3_F02:59|--82/*
if DESC_MACRORAMO $=$ 'Veículos' and PLANO $=3$ and VALOR $>=95$ and VALOR $<148$ then PID $=244$;else * $/$ veiculos_3_F02:95|--148/*

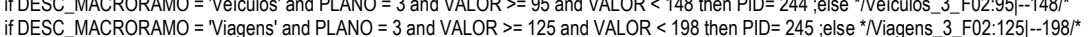

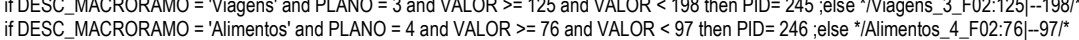

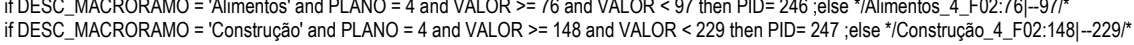
if DESC_MACRORAMO = 'Diversão' and PLANO $=4$ and VALOR $>=96$ and VALOR $<160$ then PID $=248$;else */Diversão_4_F02:96|--160/* if DESC_MACRORAMO = 'Farmácia' and PLANO $=4$ and VALOR $>=52$ and VALOR $<84$ then PID= 249 ;else */Farmácia_4_F02:52|--84/* if DESC_MACRORAMO $=$ 'Geral' and PLANO $=4$ and VALOR $>=94$ and VALOR $<144$ then PID $=250$;else */Geral_4_F02:94 $\mid-144 /{ }^{*}$ if DESC_MACRORAMO $=$ 'Joalherias' and PLANO $=4$ and VALOR $>=108$ and VALOR $<161$ then PID $=251$;else */Joalherias_4_F02:108|--161/ if DESC_MACRORAMO $=$ 'Livraria' and PLANO $=4$ and VALOR $>=87$ and VALOR $<131$ then PID $=252$;else */Livraria_4_F02:87|-131/* if DESC_MACRORAMO = 'Lojas em Geral' and PLANO $=4$ and VALOR $>=67$ and VALOR $<105$ then PID $=253$; ;ise */Lojas em Geral_4_F02:67|-105/* if DESC_MACRORAMO = 'Móveis' and PLANO $=4$ and VALOR $>=120$ and VALOR $<200$ then PID $=254$;else */Móveis_4_F02:120|--200/ if DESC_MACRORAMO = 'Perfumaria' and PLANO $=4$ and VALOR $>=99$ and VALOR $<130$ then PID $=255$;else */Perfumaria_4_F02:99|--130/* if DESC MACRORAMO = 'Postos de Gasolina' and PLANO $=4$ and VALOR $>=50$ and VALOR $<60$ then PID= 256 ;else */Postos de Gasolina 4 F02:50|--60/* if DESC_MACRORAMO = 'Serviços' and PLANO = 4 and VALOR $>=120$ and VALOR $<200$ then PID= 257 ; else */Serviços_4_F02:120| $-200{ }^{*}$

if DESC_MACRORAMO = 'Supermercado' and PLANO $=4$ and VALOR $>=39$ and VALOR $<65$ then PID $=258$; else */Supermercado 4 F F2:39|--65/ ${ }^{*}$

if DESC_MACRORAMO = 'Telecomunicação / Eletrônicos' and PLANO $=4$ and VALOR $>=99$ and VALOR $<160$ then PID $=259$;else */Telecomunicação / Eletrônicos 4 F02:99|--160/* if DESC_MACRORAMO = 'Vestuário' and PLANO $=4$ and VALOR $>=80$ and VALOR $<118$ then PID $=260$;else *Nestuário_4_F02:80|--118/*

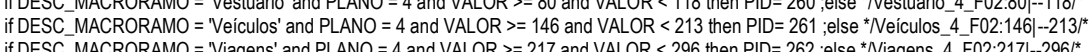
if DESC_MACRORAMO = 'Viagens' and PLANO $=4$ and VALOR $>=217$ and VALOR $<296$ then PID= 262 ;else */Viagens_4_F02:217|--296/*

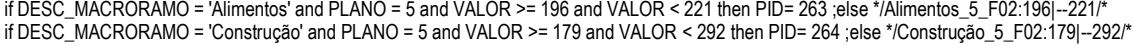
if DESC_MACRORAMO $=$ 'Construçäa' and PLANO $=5$ and VALOR $>=179$ and VALOR $<292$ then PID $=264$; else */Construção_5_F02:179|--292
if DESC_MACRORAMO $=$ 'Diversão' and PLANO $=5$ and VALOR $>=131$ and VALOR $<216$ then PID $=265$;else */Diversão 5 F02:131|-216/* if DESC_MACRORAMO = 'Diversão' and PLANO $=5$ and VALOR $>=131$ and VALOR $<216$ then PID $=265$;else */Diversão_5_F02:131|--216/*

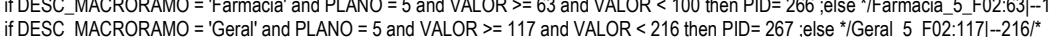
if DESC_MACRORAMO $=$ 'Geral' and PLANO $=5$ and VALOR $>=117$ and VALOR $<216$ then $\mathrm{PID}=267$;else */Geral_5_F02:117|-216/*
if DESC_MACRORAMO $=$ 'Joalherias' and PLANO $=5$ and VALOR $>=153$ and VALOR $<239$ then $\mathrm{PID}=268$;else */Joalherias 5 F02:153|-239/* if DESC_MACRORAMO $=$ 'Joalherias' and PLANO $=5$ and VALOR $>=153$ and VALOR $<239$ then PID $=268$;else */Joalherias_5_F02:153|--23
if DESC_MACRORAMO = 'Livraria' and PLANO $=5$ and VALOR $>=110$ and VALOR $<163$ then PID $=269 ;$;se */Livraria_5_F02:110|--163/* if DESC_MACRORAMO = 'Lojas em Geral' and PLANO $=5$ and VALOR $>=88$ and VALOR $<136$ then PID $=270$;else */Lojas em Geral_5_F02:88|--136/* if DESC_MACRORAMO = 'Móveis' and PLANO $=5$ and VALOR $>=150$ and VALOR $<253$ then PID $=271$; else */Móveis_5_F02:150|--253/* if DESC_MACRORAMO $=$ 'Perfumaria' and PLANO $=5$ and VALOR $>=105$ and VALOR $<154$ then PID $=272$; ;lse $* /$ Perfumaria_5_F02:105|-154/* if DESC_MACRORAMO = 'Postos de Gasolina' and PLANO $=5$ and VALOR $>=30$ and VALOR $<50$ then PID $=273$; else */Postos de Gasolina_5_F02:30|--50/* if DESC_MACRORAMO = 'Serviços' and PLANO = 5 and VALOR >= 169 and VALOR $<270$ then PID= 274 ;else */Serviços_5_F02:169|--270/* if DESC_MACRORAMO = 'Supermercado' and PLANO $=5$ and VALOR $>=49$ and VALOR $<89$ then PID $=275$;else */Supermercado_5_F02:49|--89/" if DESC_MACRORAMO = 'Telecomunicação / Eletrônicos' and PLANO = 5 and VALOR $>=119$ and VALOR $<219$ then PID= 276 ;else */Telecomunicação / Eletrônicos_5_F02:119|--219/* if DESC_MACRORAMO = 'Vestuário' and PLANO $=5$ and VALOR $>=94$ and VALOR $<139$ then PID= 277 ;else */Vestuário_5_F02:94|--139/* if DESC MACRORAMO = 'Veículos' and PLANO $=5$ and VALOR $>=193$ and VALOR $<278$ then PID $=278$;else * $/$ Veículos 5 F02:193|--278/ if DESC MACRORAMO = 'Viagens' and PLANO $=5$ and VALOR $>=248$ and VALOR $<308$ then PID $=279$;else *Viagens $5 \quad$ F02:248 $--308 /^{*}$ if DESC_MACRORAMO $=$ 'Alimentos' and PLANO $=6$ and VALOR $>=89$ and VALOR $<145$ then PID $=280$;else */Alimentos 6 F02:89|--145/* if DESC_MACRORAMO = 'Construção' and PLANO $=6$ and VALOR $>=236$ and VALOR $<392$ then PID $=281$;else */Construção_6_F02:236|-392/* if DESC_MACRORAMO $=$ 'Diversão' and PLANO $=6$ and VALOR $>=290$ and VALOR $<340$ then PID $=282$; else */Diversão 6_F02:290|--340/* if DESC_MACRORAMO = 'Farmácia' and PLANO $=6$ and VALOR $>=42$ and VALOR $<67$ then PID $=283$; ; else */Farmácia_6_F02:42|-67/* if DESC_MACRORAMO $=$ 'Farmácia' and PLANO $=6$ and VALOR $>=42$ and VALOR $<67$ then PID $=283$;else */Farmácia_6_F02:42|--6/*
if DESC_MACRORAMO $=$ 'Geral' and PLANO $=6$ and VALOR $>=123$ and VALOR $<250$ then PID $=284 ;$ else */Geral 6 F02:123|-250/* if DESC_MACRORAMO $=$ 'Geral' and PLANO $=6$ and VALOR $>=123$ and VALOR $<250$ then PID $=284$;else */Geral_6_FF2:123|-250/*
if DESC_MACRORAMO $=$ 'Joalherias' and PLANO $=6$ and VALOR $>=200$ and VALOR $<298$ then PID $=285 ;$; else */Joalherias_6_F02:200|--298/* if DESC_MACRORAMO $=$ 'Joalherias' and PLANO $=6$ and VALOR $>=200$ and VALOR $<298$ then PID $=285$;else */Joalherias_6_F02:200|--298r
if DESC_MACRORAMO $=$ 'Livraria' and PLANO $=6$ and VALOR $>=137$ and VALOR $<214$ then PID $=286$;else */Livraria 6 F02:137|-214/*

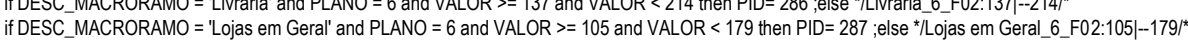
if DESC_MACRORAMO $=$ 'Lojas em Geral' and PLANO $=6$ and VALOR $>=105$ and VALOR $<179$ then PID $=287$; else */Lojas em Geral_6 6 -
if DESC_MACRORAMO $=$ 'Móveis' and PLANO $=6$ and VALOR $>=208$ and VALOR $<322$ then PID $=288$ else */Móveis 6 F02:208|--322/*

if DESC_MACRORAMO $=$ 'Móveis' and PLANO $=6$ and VALOR $>=208$ and VALOR $<322$ then PID $=288$;else */Móveis_6_F02:208|--322/*
if DESC_MACRORAMO $=$ 'Perfumaria' and PLANO $=6$ and VALOR $>=140$ and VALOR $<212$ then PID 289 ;else */Perfumaria 6 F02:140|-212

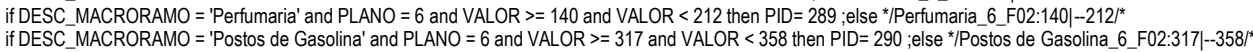
if DESC_MACRORAMO = 'Serviços' and PLANO $=6$ and VALOR $>=167$ and VALOR $<320$ then PID $=291$;else */Serviços_6_F02:167|--320/* if DESC_MACRORAMO = 'Supermercado' and PLANO $=6$ and VALOR $>=43$ and VALOR $<71$ then PID $=292$;else */Supermercado_6_F02:43|--71/ 
if DESC_MACRORAMO = 'Telecomunicação / Eletrônicos' and PLANO = 6 and VALOR >= 149 and VALOR <259 then PID= 293 ;else */Telecomunicação / Eletrônicos_6_F02:149|--259/* if DESC_MACRORAMO = 'Vestuário' and PLANO $=6$ and VALOR $>=99$ and VALOR $<151$ then PID $=294$;else *Nestuário_6_F02:99|--151/* if DESC_MACRORAMO = 'Veículos' and PLANO $=6$ and VALOR $>=209$ and VALOR $<330$ then PID $=295$;else * $/$ Veículos_6_F02:209|--330/* if DESC_MACRORAMO $=$ 'Viagens' and PLANO $=6$ and VALOR $>=384$ and VALOR $<517$ then PID $=296$; else * ${ }^{*}$ Viagens 6 F $F 2: 384 \mid--5177^{*}$ if DESC_MACRORAMO = 'Construção' and PLANO $=7$ and VALOR $>=250$ and VALOR $<347$ then PID $=297 ;$;else */Construção_7_F02:250|--347/*

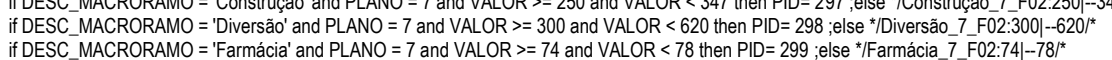

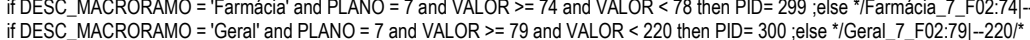
if DESC_MACRORAMO $=$ 'Geral' and PLANO $=7$ and VALOR $>=79$ and VALOR $<220$ then PID $=300$;else */Geral_7_FO2:79|--220/*
if DESC_MACRORAMO $=$ 'Joalherias' and PLANO $=7$ and VALOR $>=220$ and VALOR $<307$ then PID $=301$;else */Joalherias_7_FO2:220|--307/* if DESC_MACRORAMO = 'Joalherias' and PLANO $=7$ and VALOR $>=220$ and VALOR $<307$ then PID $=301$;else */Joalherias_7_FO2:220|--307"
if DESC_MACRORAMO = 'Livraria' and PLANO $=7$ and VALOR $>=156$ and VALOR $<209$ then PID $=302$;else */Livraria 7 F02:156|--209/* if DESC_MACRORAMO = 'Lojas em Geral' and PLANO $=7$ and VALOR $>=97$ and VALOR $<187$ then PID $=303$; else */Lojas em Geral_7_F02:97|--187/* if DESC_MACRORAMO = 'Móveis' and PLANO $=7$ and VALOR >= 219 and VALOR < 374 then PID= 304 ;else */Móveis_7_F02:219|--374/* if DESC_MACRORAMO $=$ 'Perfumaria' and PLANO $=7$ and VALOR $>=100$ and VALOR $<135$ then PID $=305$; ; 1 se ${ }^{*} /$ Perfumaria_7 F02:100|- $135 /^{*}$ if DESC_MACRORAMO = 'Serviços' and PLANO = 7 and VALOR $>=79$ and VALOR $<363$ then PID = 306 ;else */Serviços_7_F02:79|--363/* if DESC_MACRORAMO = 'Supermercado' and PLANO $=7$ and VALOR $>=70$ and VALOR $<114$ then PID $=307 ;$; else $* /$ Supermercado_7_F02:70|--114/* if DESC_MACRORAMO = 'Telecomunicação / Eletrônicos' and PLANO $=7$ and VALOR $>=183$ and VALOR $<301$ then PID $=308$;else */T/Telecomunicação / Eletrônicos_7_F02:183|--301/ ${ }^{*}$ if DESC_MACRORAMO = 'Vestuário' and PLANO $=7$ and VALOR $>=97$ and VALOR $<181$ then PID $=309$;else * Nestuário_7_F02:97|--181/* if DESC_MACRORAMO = 'Veículos' and PLANO $=7$ and VALOR $>=157$ and VALOR $<259$ then PID $=310$; if DESC_MACRORAMO = 'Viagens' and PLANO $=7$ and VALOR $>=322$ and VALOR $<325$ then PID $=311$;else */Viagens_7 _F02:322|--325/* if DESC_MACRORAMO = 'Construção' and PLANO = 8 and VALOR >= 359 and VALOR $<551$ then PID= 312 ;else */Construção_8_F02:359|--551/* if DESC_MACRORAMO = 'Diversão' and PLANO $=8$ and VALOR $>=560$ and VALOR $<560$ then PID $=313$;else */Diversão_8_F02:560|--560/* if DESC_MACRORAMO = 'Farmácia' and PLANO $=8$ and VALOR $>=81$ and VALOR $<93$ then PID= 314 ;else */Farmácia 8 F02:81|--93/* if DESC_MACRORAMO $=$ 'Geral' and PLANO $=8$ and VALOR $>=182$ and VALOR $<262$ then PID $=315$; ; lse */Geral 8 F $02: 182 \mid-2622^{*}$ if DESC_MACRORAMO $=$ 'Joalherias' and PLANO $=8$ and VALOR $>=345$ and VALOR $<440$ then PID $=316$;else $* / J$ Joalherias_8_F02:345|--440/* if DESC_MACRORAMO $=$ 'Livraria' and PLANO $=8$ and VALOR $>=100$ and VALOR $<206$ then PID $=317$; else */Livraria_8_FO2:100|--206/* if DESC_MACRORAMO = 'Lojas em Geral' and PLANO $=8$ and VALOR $>=123$ and VALOR $<250$ then PID $=318$ else */Lojas em Geral_ 8 F02:123|-250/* if DESC_MACRORAMO = 'Ḿ́,

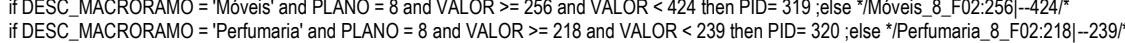

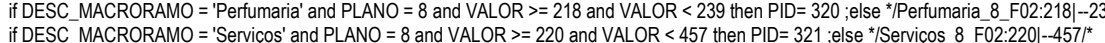
if DESC_MACRORAMO $=$ 'ServiçOS' and PLANO $=8$ and VALOR $>=220$ and VALOR $<457$ then PID $=321$; ;se */Serviços_8_F02:220|--457/*
if DESC_MACRORAMO $=$ 'Supermercado' and PLANO $=8$ and VALOR $>=89$ and VALOR $<158$ then PID $=322 ;$;else */Supermercado_8_F02:89|-158/* if DESC_MACRORAMO = 'Supermercado' and PLANO $=8$ and VALOR $>=89$ and VALOR $<158$ then PID $=322$;else */Supermercado_8_F02:89|--158/ ${ }^{*}$ if DESC_MACRORAMO $=$ 'Telecomunicação / Eletrônicos' and PLANO $=8$ and VALOR $>=209$ and VALOR $<336$ then PID $=323$;else */Telecom
if DESC_MACRORAMO $=$ 'Vestuário' and PLANO $=8$ and VALOR $>=115$ and VALOR $<174$ then PID $=324$;else *Nestuário 8 F02:115|--174/* if DESC_MACRORAMO = 'Veículos' and PLANO $=8$ and VALOR $>=264$ and VALOR $<499$ then PID $=325$;else *Neículos_8_F02:264|--499" if DESC_MACRORAMO $=$ 'Viagens' and PLANO $=8$ and VALOR $>=408$ and VALOR $<658$ then PID $=326$;else */Viagens_8_F02:408|--658/ if DESC_MACRORAMO = 'Construção' and PLANO = 9 and VALOR >= 416 and VALOR < 695 then PID = 327; ;else */Construção_9_F02:416|--695/ if DESC_MACRORAMO = 'Diversão' and PLANO $=9$ and VALOR $>=337$ and VALOR $<337$ then PID $=328$; else */Diversão_9_F02:337|--337/* if DESC_MACRORAMO $=$ 'Farmácia' and PLANO $=9$ and VALOR $>=92$ and VALOR $<95$ then PID $=329$;else */Farmácia_9_F02:92|--95/* if DESC_MACRORAMO $=$ 'Geral' and PLANO $=9$ and VALOR $>=94$ and VALOR $<97$ then PID $=330$;else $/ /$ Geral_9_F02:94|--97/* if DESC_MACRORAMO = 'Joalherias' and PLANO $=9$ and VALOR $>=98$ and VALOR $<208$ then PID $=331$; else */Joalherias 9 F02:98|--208/* if DESC_MACRORAMO = 'Livraria' and PLANO $=9$ and VALOR $>=97$ and VALOR $<242$ then PID $=332$; ; if DESC_MACRORAMO = 'Lojas em Geral' and PLANO $=9$ and VALOR $>=99$ and VALOR $<219$ then PID $=333$;else */Lojas em Geral_9_F02:99|--219/* if DESC_MACRORAMO = 'Móveis' and PLANO $=9$ and VALOR $>=376$ and VALOR $<556$ then PID $=334$;else */Móveis_9_F02:376|--556/* if DESC_MACRORAMO = 'Serviços' and PLANO = 9 and VALOR $>=132$ and VALOR $<292$ then PID= 335 ; ;lse */Serviços_9_F02:132|-292/* if DESC_MACRORAMO = 'Supermercado' and PLANO $=9$ and VALOR $>=74$ and VALOR $<149$ then PID= 336 ;else */Supermercado 9 F02:74|-149/* if DESC_MACRORAMO = 'Telecomunicação / Eletrônicos' and PLANO $=9$ and VALOR >= 134 and VALOR <282 then PID $=337$; else */Telecomunicação / Eletrônicos_9_F02:134|--282/* if DESC_MACRORAMO = 'Vestuário' and PLANO $=9$ and VALOR $>=95$ and VALOR $<99$ then PID $=338$; else */Vestuário_9_F02:95|--99/* if DESC_MACRORAMO $=$ 'Veículos' and PLANO $=9$ and VALOR $>=480$ and VALOR $<480$ then PID $=339$; ; else * $*$ Veículos_9_F02:480|--480/* if DESC_MACRORAMO $=$ 'Veiculos' and PLANO $=9$ and VALOR $>=480$ and VALOR $<480$ then PID $=339$;else $*$ Veiculos_9_F02:480|--480" if DESC_MACRORAMO $=$ 'Viagens' and PLANO $=9$ and VALOR $>=466$ and VALOR $<520$ then PID $=340$;else * $/$ iagens_9_F02:466|--520/*
if DESC_MACRORAMO $=$ 'Alimentos' and PLANO $=10$ and VALOR $>=108$ and VALOR $<108$ then PID $=341$;else $* /$ Alimentos_10_F02:108|--108/* if DESC_MACRORAMO $=$ 'Alimentos' and PLANO $=10$ and VALOR $>=108$ and VALOR $<108$ then PID $=341$;else */Alimentos_10_F02:108|--108/*
if DESC_MACRORAMO $=$ 'Construção' and PLANO $=10$ and VALOR $>=350$ and VALOR $<600$ then PID $=342 ;$;else $* /$ Construçẫo_10_F02:350|---600/ if DESC_MACRORAMO = 'Construção' and PLANO $=10$ and VALOR $>=350$ and VALOR $<600$ then PID $=342$; else */Construção_10_FO2:350|--600
if DESC_MACRORAMO = 'Diversão' and PLANO $=10$ and VALOR $>=234$ and VALOR $<300$ then PID $=343$;else */Diversão_10_F02:234|-300/*

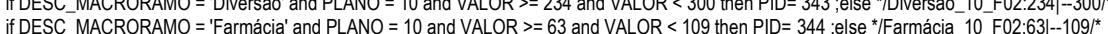

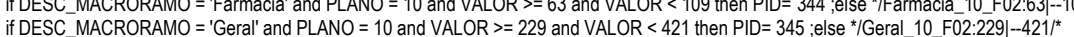
if DESC_MACRORAMO $=$ 'Geral' and PLANO $=10$ and VALOR $>=229$ and VALOR $<421$ then PID $=345$;else */Geral_10_F02:229|--421/*
if DESC_MACRORAMO $=$ 'Joalherias' and PLANO $=10$ and VALOR $>=300$ and VALOR $<450$ then PID $=346$;else $/ /$ Joalherias $10 \quad F 02: 300 \mid-450 /{ }^{*}$ if DESC_MACRORAMO $=$ 'Joalherias' and PLANO $=10$ and VALOR $>=300$ and VALOR $<450$ then PID $=346$; else $* / J$ Joalherias_10_F02:300|--450
if DESC MACRORAMO $=$ 'Livraria' and PLANO $=10$ and VALOR $>=126$ and VALOR $<295$ then PID $=347$;else */Livraria 10 F02:126 $--295 /^{*}$ if DESC_MACRORAMO $=$ 'Livraria' and PLANO $=10$ and VALOR $>=126$ and VALOR $<295$ then PID $=347$;else */Livraria_10_F2:126|--295/*
if DESC_MACRORAMO $=$ 'Lojas em Geral' and PLANO $=10$ and VALOR $>=149$ and VALOR $<259$ then PID $=348 ;$;else */Lojas em Geral_10_F02:149|-259/* if DESC_MACRORAMO = 'Móveis' and PLANO = 10 and VALOR > 299 and VALOR < 499 then PID = 349 ;else */Móveis_10_F02:299|--499/ if DESC_MACRORAMO $=$ 'Perfumaria' and PLANO $=10$ and VALOR $>=148$ and VALOR $<319$ then PID $=350$; else */Perfumaria_10_F02:148|--319/* if DESC_MACRORAMO $=$ 'Postos de Gasolina' and PLANO $=10$ and VALOR $>=303$ and VALOR $<454$ then PID $=351$;else */Postos de Gasolina_10_F02:303|--454/* if DESC_MACRORAMO = 'Serviços' and PLANO $=10$ and VALOR > 249 and VALOR < 478 then PID= 352 ;else */Serviços 10 F02:249|--478

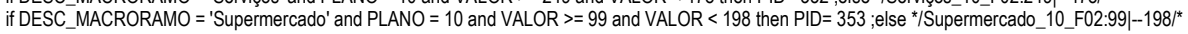
if DESC_MACRORAMO = 'Telecomunicação / Eletrônicos' and PLANO = 10 and VALOR >= 199 and VALOR < 350 then PID= 354 ;else */Telecomunicação / Eletrônicos_10_F02:199|--350/* if DESC_MACRORAMO = 'Vestuário' and PLANO $=10$ and VALOR $>=134$ and VALOR $<199$ then PID $=355$;else * Nestuário_10_FO2:134|--1999/* if DESC_MACRORAMO = 'Veiculos' and PLANO $=10$ and VALOR $>=242$ and VALOR $<418$ then PID $=356$;else *Neículos 10 F02:242|--418/* if DESC MACRORAMO $=$ 'Viagens' and PLANO $=10$ and VALOR $>=297$ and VALOR $<558$ then PID $=357$; else */Viagens 10 F02:297|--558/* if DESC_MACRORAMO = 'Geral' and PLANO $=11$ and VALOR $>=220$ and VALOR $<220$ then PID $=358$;else */Geral 11 F02:220|--220/* if DESC MACRORAMO = 'Livraria' and PLANO = 11 and VALOR >= 116 and VALOR $<296$ then PID = 359 ; else */Livraria 11 F02:116|--296/* if DESC_MACRORAMO $=$ 'Lojas em Geral' and PLANO $=11$ and VALOR $>=341$ and VALOR $<465$ then PID $=360$;else */Lojas em Geral_11_F02:341|--465/* if $\mathrm{DESC}$ MACRORAMO $=$ 'Móveis' and PLANO $=11$ and VALOR $>=300$ and VALOR $<530$ then PID $=361$; else * $*$ Móveis_11_F02:300|- $-530^{*}$ if DESC_MACRORAMO = 'Supermercado' and PLANO $=11$ and VALOR $>=99$ and VALOR $<205$ then PID $=362$; else * $/$ Supermercado 11 F02:99|--205/* if DESC_MACRORAMO $=$ 'Supermercado' and PLANO $=11$ and VALOR $>=99$ and VALOR $<205$ then PID $=362$;else */Supermercado_11_FO2:99|--205/*
if DESC_MACRORAMO $=$ 'Telecomunicacãão / Eletrônicos' and PLANO $=11$ and VALOR $>=588$ and VALOR $<606$ then PID $=363$;else */Telecomunicação / Eletrônicos 11 F02:588|--606/* If DESC_MACRORAMO $=$ 'Telecomunicação/ Eletrônicos' and PLANO $=11$ and VALOR $>=588$ and VALOR $<606$ then PID $=363 ;$;else */Telecomun
if DESC_MACRORAMO $=$ 'Vestuário' and PLANO $=11$ and VALOR $>=166$ and VALOR $<223$ then PID $=364 ;$;else *Nestuário_11_F02:166|--223/*

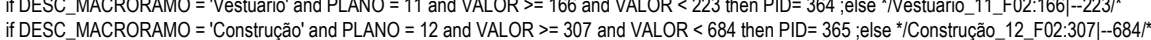
if DESC_MACRORAMO $=$ 'Construção' and PLANO $=12$ and VALOR $>=307$ and VALOR $<684$ then PID $=365$;else */Construção_12_F02:307|--684" if DESC_MACRORAMO $=$ 'Geral' and PLANO $=12$ and VALOR $>=327$ and VALOR $<509$ then PID $=367 \cdot$ else */Geral 12 F02:327|--509/* if DESC_MACRORAMO $=$ 'Geral' and PLANO $=12$ and VALOR $>=327$ and VALOR $<509$ then PID $=367$;else */Geral_12_F02:327|--509/*
if DESC_MACRORAMO = 'Joalherias' and PLANO $=12$ and VALOR $>=300$ and VALOR $<502$ then PID $=368$ else*/Joalherias 12 F02:300 $--502 / *$ if DESC_MACRORAMO $=$ 'Joalherias' and PLANO $=12$ and VALOR $>=300$ and VALOR $<502$ then PID $=368$;else */Joalherias_12_F02:300|--502"
if DESC_MACRORAMO = 'Livraria' and PLANO $=12$ and VALOR $>=132$ and VALOR $<249$ then PID $=369$;else */Livraria 12_F02:132|-249/* if DESC_MACRORAMO $=$ 'Livraria' and PLANO $=12$ and VALOR $>=132$ and VALOR $<249$ then PID $=369$;else */Livraria_12_F02:132|--249/*
if DESC_MACRORAMO $=$ 'Lojas em Geral' and PLANO $=12$ and VALOR $>=215$ and VALOR $<442$ then PID $=370$;else */Lojas em Geral_12_F02:215|--442/*

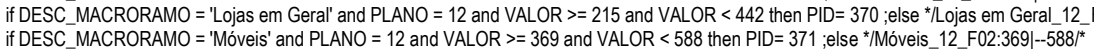
if DESC_MACRORAMO = 'Perfumaria' and PLANO $=12$ and VALOR $>=95$ and VALOR $<295$ then PID $=372$; else $* /$ Perfumaria_12_F02:95|--295/ if DESC_MACRORAMO = 'Serviços' and PLANO = 12 and VALOR $>=205$ and VALOR $<358$ then PID= 373 ;else */Serviços_12_F02:205|--358/* if DESC_MACRORAMO = 'Supermercado' and PLANO $=12$ and VALOR $>=168$ and VALOR $<346$ then PID $=374 ;$;else */Supermercado_12_F02:168|--346/* if DESC_MACRORAMO = 'Telecomunicação / Eletrônicos' and PLANO $=12$ and VALOR $>=319$ and VALOR $<500$ then PID $=375$;else */Telecomunicação / Eletrônicos_12_F02:319|--500/* if DESC_MACRORAMO = 'Vestuário' and PLANO $=12$ and VALOR $>=189$ and VALOR $<259$ then PID = 376 ;else *Nestuário_12_F02:189|-259/* if DESC_MACRORAMO = 'Veículos' and PLANO $=12$ and VALOR $>=310$ and VALOR $<430$ then PID $=377$;else *Neículos 12 F02:310|- -430 /* if DESC MACRORAMO $=$ 'Viagens' and PLANO $=12$ and VALOR $>=370$ and VALOR $<589$ then PID $=378$; else * $N$ Viagens $12 \quad$ F02:370|--589/* if DESC_MACRORAMO = 'Supermercado' and PLANO $=13$ and VALOR $>=598$ and VALOR $<787$ then PID $=379$;else */Supermercado_13_F02:598|--787/* if DESC MACRORAMO $=$ 'Lojas em Geral' and PLANO $=14$ and VALOR $>=577$ and VALOR $<577$ then PID $=380$ else */Lojas em Geral 14 F02:577|--577/* if DESCMACRORAMO = 'Supermat if DESC_MACRORAMO $=$ 'Construção' and PLANO $=15$ and VALOR $>=228$ and VALOR $<228$ then PID $=382$; else */Construção 15 F02:228|--228/*

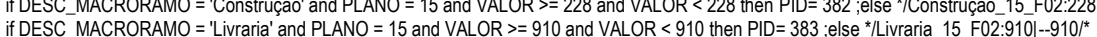
if DESC_MACRORAMO $=$ 'Livraria' and PLANO $=15$ and VALOR $>=910$ and VALOR $<910$ then PID $=383$;else */Livraria_15_FO2:910|--910/*
if DESC_MACRORAMO $=$ 'Lojas em Geral' and PLANO $=15$ and VALOR $>=159$ and VALOR $<178$ then PID $=384$;else */Lojas em Geral 15 F02:159|--178/* if DESC_MACRORAMO $=$ 'Lojas em Geral' and PLANO $=15$ and VALOR $>=159$ and VALOR $<178$ then PID $=384$;else */Lojas em Geral_15_FO2:159|--178/*
if DESC_MACRORAMO $=$ 'Supermercado' and PLANO $=15$ and VALOR $>=248$ and VALOR $<548$ then PID $=385$ eelse */Supermercado 15 F02:248|--548/* if DESC_MACRORAMO = 'Supermercado' and PLANO $=15$ and VALOR $>=248$ and VALOR $<548$ then PID $=385$;else */Supermercado_15_F02:248|--548/*
if DESC_MACRORAMO = 'Telecomunicação / Eletrônicos' and PLANO $=15$ and VALOR $>=1415$ and VALOR $<1415$ then PID $=386$;else */Telecomunicação / Eletrônicos_15_F02:1415|--

if DESC_MACRORAMO = 'Supermercado' and PLANO $=18$ and VALOR $>=388$ and VALOR $<762$ then PID $=387$;else */Supermercado_18_F02:388|--762/ ${ }^{*}$

if DESC_MACRORAMO = 'Supermercado' and PLANO = 24 and VALOR $>=158$ and VALOR $<243$ then PID = 388 ;else */Supermercado_24_F02:158|--243/*

if DESC_MACRORAMO = 'Alimentos' and PLANO $=1$ and VALOR $>=21$ and VALOR $<39$ then PID = 389 ;else */Alimentos_1_F03:21|--39/*

if DESC_MACRORAMO $=$ 'Construção' and PLANO $=1$ and VALOR $>=26$ and VALOR $<47$ then PID $=390$;else */Construção_1_F03:26|--47/*

if DESC_MACRORAMO $=$ 'Diversão' and PLANO $=1$ and VALOR $>=19$ and VALOR $<31$ then PID $=391$;else */Diversão_1_F03:19|--31/*
if DESC_MACRORAMO = 'Farmácia' and PLANO $=1$ and VALOR $>=17$ and VALOR $<27$ then PID $=392$;else */Farmácia_1_F03:17|-27/ 
if DESC_MACRORAMO $=$ 'Geral' and PLANO $=1$ and VALOR $>=23$ and VALOR $<38$ then PID $=393$;else */Geral_1_F03:23|-38/ ${ }^{*}$

if DESC MACRORAMO = 'Joalherias' and PLANO $=1$ and VALOR $>=19$ and VALOR $<30$ then PID $=394$;else */Joalherias 1 F03:19|--30/*

if DESC MACRORAMO = 'Livraria' and PLANO = 1 and VALOR $>=22$ and VALOR $<36$ then PID $=395$;else */Livraria 1 F03.221-36/*

if DESC_MACRORAMO = 'Lojas em Geral' and PLANO $=1$ and VALOR $>=21$ and VALOR $<37$ then PID $=396$;else */Lojas em Geral_1_F03:21|--37/*

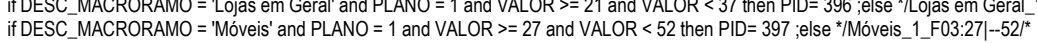

if DESC_MACRORAMO $=$ 'Móveis' and PLANO $=1$ and VALOR $>=27$ and VALOR $<52$ then PID $=397$;else */Móveis_1_F03:27|--52/*
if DESC_MACRORAMO $=$ 'Perfumaria' and PLANO $=1$ and VALOR $>=21$ and VALOR $<36$ then PID $=398$;else */Perfumaria_1_F03:21|--36/*

if DESC_MACRORAMO $=$ 'Perfumaria' and PLANO $=1$ and VALOR $>=21$ and VALOR $<36$ then PID $=398 ;$ else */Perfumaria_1_FO3:21|--36/*
if DESC_MACRORAMO $=$ 'Postos de Gasolina' and PLANO $=1$ and VALOR $>=30$ and VALOR $<50$ then PID $=399 ;$;lse */Postos de Gasolina_1_F03:30|--50/*

if DESC_MACRORAMO $=$ 'Postos de Gasolina' and PLANO $=1$ and VALOR $>=30$ and VALOR $<50$ then PID $=399$;else */Postos de Gasolity
if DESC_MACRORAMO $=$ 'Serviços' and PLANO $=1$ and VALOR $>=30$ and VALOR $<50$ then PID $=400 ;$;else */Serviços_1_F03:30|--50/*

if DESC_MACRORAMO = 'Serviços' and PLANO $=1$ and VALOR $>=30$ and VALOR $<50$ then PID $=400$;else */Serviços_1_FO3:30|--50/*
if DESC_MACRORAMO = 'Supermercado' and PLANO $=1$ and VALOR $>=28$ and VALOR $<61$ then PID $=401$;else */Supermercado 1 F03:28|--61/*

if DESC_MACRORAMO $=$ 'Supermercado' and PLANO $=1$ and VALOR $>=28$ and VALOR $<61$ then PID $=401$;else */Supermercado_1_F03:28|--61/ ${ }^{*}$
if DESC_MACRORAMO $=$ 'Telecomunicação / Eletrônicos' and PLANO $=1$ and VALOR $>=20$ and VALOR $<29$ then PID $=402$;else */Telecomunicação / Eletrônicos_1_F03:20|--29/*

if DESC_MACRORAMO $=$ 'Telecomunicação / Eletrônicos' and PLANO $=1$ and VALOR $>=20$ and VALOR $<29$ then PID $=402$; else */Telecomu

if DESC_MACRORAMO $=$ 'Vestuário' and PLANO $=1$ and VALOR $>=24$ and VALOR $<38$ then PID $=403 ;$;else *Nestuário_1_F03:24|--38/*
if DESC_MACRORAMO $=$ 'Veículos' and PLANO $=1$ and VALOR $>=27$ and VALOR $<50$ then PID $=404$;else *Neículos_1_F03:27|--50/*

if DESC_MACRORAMO $=$ 'Veículos' and PLANO $=1$ and VALOR $>=27$ and VALOR $<50$ then PID $=404 ;$;else * $/$ eículos_1_FO3:27|--50/
if DESC_MACRORAMO $=$ 'Viagens' and PLANO $=1$ and VALOR $>=32$ and VALOR $<51$ then PID $=405 ;$ else * Niagens_1_F03:32|-51/*

if DESC_MACRORAMO $=$ 'Alimentos' and PLANO $=2$ and VALOR $>=75$ and VALOR $<116$ then PID $=406$;else */Alimentos_2_F03:75|--116/*

if DESC_MACRORAMO = 'Construção' and PLANO $=2$ and VALOR $>=74$ and VALOR $<122$ then PID = 407 ; else */Construção_2_F03:74|--122/

if DESC_MACRORAMO $=$ 'Diversão' and PLANO $=2$ and VALOR $>=64$ and VALOR $<92$ then PID $=408$; else */Diversão_2_F03:64|--92/*

if DESC_MACRORAMO = 'Farmácia' and PLANO $=2$ and VALOR $>=38$ and VALOR $<54$ then PID $=409$;else */Farmácia_2_F03:38|--54/

if DESC_MACRORAMO $=$ 'Geral' and PLANO $=2$ and VALOR $>=56$ and VALOR $<85$ then PID $=410$;else $/ / G$ eral_2_F03:56|--85/*

if DESC_MACRORAMO = 'Joalherias' and PLANO $=2$ and VALOR $>=50$ and VALOR $<79$ then PID= 411 ; ;lse */Joalherias_2_F03:50|-79/*

if DESC_MACRORAMO = 'Livraria' and PLANO $=2$ and VALOR $>=51$ and VALOR $<72$ then PID $=412$;else */Livraria_2_F03:51 $|-72|^{*}$

if DESC_MACRORAMO = 'Lojas em Geral' and PLANO $=2$ and VALOR $>=49$ and VALOR $<70$ then PID $=413$; else */Lojas em Geral_2 F03:49|--70/*

if DESC_MACRORAMO = 'Móveis' and PLANO $=2$ and VALOR $>=64$ and VALOR $<115$ then PID= 414 ;else */Móveis_2_F03:64|-115/*

if DESC_MACRORAMO = 'Perfumaria' and PLANO $=2$ and VALOR $>=49$ and VALOR $<67$ then PID $=415$;

if DESC_MACRORAMO = 'Postos de Gasolina' and PLANO $=2$ and VALOR $>=50$ and VALOR $<75$ then PID $=416$; else */Postos de Gasolina_2_F03:50|-75/*

if DESC_MACRORAMO = 'Serviços' and PLANO $=2$ and VALOR $>=67$ and VALOR $<106$ then PID $=417$; ;else */Serviços_2_F03:67|--106/*

if DESC_MACRORAMO = 'Supermercado' and PLANO $=2$ and VALOR $>=35$ and VALOR $<55$ then $\mathrm{PID}=418$; else */Supermercado_ 2 F03:35|--55/*

if DESC_MACRORAMO $=$ 'Supermercado' and PLANO $=2$ and VALOR $>=35$ and VALOR $<55$ then PID $=418$; ; else */Supermercado_2_F03:35|--55/*
if DESC_MACRORAMO $=$ 'Telecomunicação / Eletrônicos' and PLANO $=2$ and VALOR $>=60$ and VALOR $<99$ then PID $=419$;else */Telecomunicação / Eletrônicos_2_F03:60|--99/*

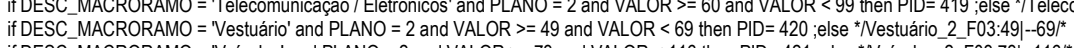

作

if DESC_MACRORAMO $=$ 'Viagens' and PLANO $=2$ and VALOR $>=112$ and VALOR $<175$ then PID $=422 ;$;else * $/$ Viagens_2_F03:112|--175/*

if DESC_MACRORAMO $=$ 'Construção' and PLANO $=3$ and VALOR $>=150$ and VALOR $<256$ then PID $=424$; else */Construção_3_Fo3:150|-256/ ${ }^{*}$

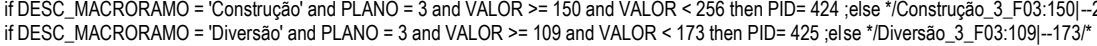

if DESC_MACRORAMO = 'Farmácia' and PLANO $=3$ and VALOR $>=60$ and VALOR $<87$ then PID $=426$;else */Farmácia_3_F03:60| --87

if DESC_MACRORAMO $=$ 'Geral' and PLANO $=3$ and VALOR $>=110$ and VALOR $<191$ then PID $=427$;else */Geral_3_F03:110|-191/*

if DESC_MACRORAMO $=$ 'Joalherias' and PLANO $=3$ and VALOR $>=99$ and VALOR $<154$ then PID $=428$;else */Joalherias_3_FO3:99|--154/*

if DESC_MACRORAMO = 'Livraria' and PLANO $=3$ and VALOR $>=90$ and VALOR $<133$ then PID $=429$; else */Livraria_3_F03:90|--133/*

if DESC_MACRORAMO = 'Lojas em Geral' and PLANO $=3$ and VALOR $>=82$ and VALOR $<129$ then PID $=430$;else */Lojas em Geral_3_F03:82|-129/*

if DESC_MACRORAMO = 'Móveis' and PLANO = 3 and VALOR >= 149 and VALOR $<269$ then PID= 431 ; else */Móveis_3_F03:149|--269/*

if DESC_MACRORAMO = 'Perfumaria' and PLANO $=3$ and VALOR $>=89$ and VALOR $<119$ then PID= 432 ;else */Perfumaria_3_F03:89|--119/*

if DESC_MACRORAMO = 'Postos de Gasolina' and PLANO $=3$ and VALOR $>=60$ and VALOR < 90 then PID= 433 ; else */Postos de Gasolina 3 F F03:60|--90/*

if DESC_MACRORAMO = 'Serviços' and PLANO $=3$ and VALOR $>=137$ and VALOR $<221$ then PID $=434$;else */Serviços_3_F03:137|--221/*

if DESC_MACRORAMO = 'Supermercado' and PLANO $=3$ and VALOR $>=44$ and VALOR $<80$ then PID $=435$;else */Supermercado_3_F03:44|--80/*

if DESC_MACRORAMO = 'Telecomunicação / Eletrônicos' and PLANO = 3 and VALOR $>=129$ and VALOR $<214$ then PID= 436 ; else */Telecomunicação / Eletrônicos_ 3 _F03:129|--214/*

if DESC_MACRORAMO = 'Vestuário' and PLANO $=3$ and VALOR $>=82$ and VALOR $<120$ then PID $=437$; else *Nestuário_3_F03:82|--120/*

if DESC_MACRORAMO $=$ 'Veículos' and PLANO $=3$ and VALOR $>=148$ and VALOR $<227$ then PID $=438$;else * $/$ Veículos_3_F03:148|-227/*

if DESC_MACRORAMO $=$ 'Viagens' and PLANO $=3$ and VALOR $>=198$ and VALOR $<337$ then PID $=439$; else * Niagens_3_F03:198|--337/*

if DESC_MACRORAMO $=$ 'Alimentos' and PLANO $=4$ and VALOR $>=97$ and VALOR $<185$ then PID $=440$; else */Alimentos_4_F03:97|--185/*

if DESC_MACRORAMO = 'Construção' and PLANO $=4$ and VALOR $>=229$ and VALOR $<384$ then PID $=441$; else */Construção_4_F03:229|--384/*

if DESC_MACRORAMO $=$ 'Construção' and PLANO $=4$ and VALOR $>=229$ and VALOR $<384$ then PID $=441$; else */Construção_4_FO3:229|--384
if DESC_MACRORAMO $=$ 'Diversão' and PLANO $=4$ and VALOR $>=160$ and VALOR $<244$ then PID $=442$;else */Diversão__F03:160|- $-244 /^{*}$

if DESC_MACRORAMO = 'Farmácia' and PLANO $=4$ and VALOR $>=84$ and VALOR $<133$ then PID $=443$; else */Farmácia_4_F03:84|-133"

if DESC_MACRORAMO = 'Geral' and PLANO $=4$ and VALOR > 144 and VALOR $<272$ then PID $=444$;else */Geral_4_F03:144|-272/

if DESC_MACRORAMO $=$ 'Joalherias' and PLANO $=4$ and VALOR $>=161$ and VALOR $<253$ then PID $=445$; else */Joalherias_4_F03:161|-253/

if DESC_MACRORAMO = 'Livraria' and PLANO $=4$ and VALOR > $>131$ and VALOR $<215$ then PID= 446 ;else */Livraria_4_F03:131|-215/"

if DESC_MACRORAMO $=$ 'Lojas em Geral' and PLANO $=4$ and VALOR $>=105$ and VALOR $<178$ then PID $=447$; ;else */Lojas em Geral_4_F03:105|-178/

if DESC_MACRORAMO = 'Móveis' and PLANO $=4$ and VALOR $>=200$ and VALOR $<322$ then PID $=448$;else */Móveis_4_F03:200|--322/ ${ }^{*}$

if DESC_MACRORAMO = 'Perfumaria' and PLANO $=4$ and VALOR $>=130$ and VALOR $<168$ then PID= 449 ;else */Perfumaria_4_F03:130|--168/*

if DESC_MACRORAMO = 'Postos de Gasolina' and PLANO $=4$ and VALOR $>=60$ and VALOR $<80$ then PID $=450 ;$; else */Postos de Gasolina_4_F03:60|--80/*

if DESC_MACRORAMO = 'Serviços' and PLANO $=4$ and VALOR $>=200$ and VALOR $<360$ then PID $=451$; else */Serviços_4_F03:200|--360/

if DESC_MACRORAMO = 'Supermercado' and PLANO $=4$ and VALOR $>=65$ and VALOR $<110$ then PID $=452$;else */Supermercado 4 F03:65|--110/"

if DESC_MACRORAMO = 'Telecomunicação / Eletrônicos' and PLANO $=4$ and VALOR $>=160$ and VALOR $<279$ then PID $=453$; else $* / \bar{T}$ elecomunicação / Eletrônicos_4_F03:160|--279/*

if DESC_MACRORAMO = 'Vestuário' and PLANO $=4$ and VALOR $>=118$ and VALOR $<178$ then PID $=454$;else * Nestuário_4_F03:118|--178/*

if DESC_MACRORAMO = 'Veículos' and PLANO $=4$ and VALOR $>=213$ and VALOR $<335$ then PID $=455$;else */Veiculos $4 \_$F03:213|- $-335 / *$

if DESC_MACRORAMO = 'Viagens' and PLANO $=4$ and VALOR $>=296$ and VALOR $<425$ then PID $=456$; else */Viagens 4 F F03:296 $\mid-425 / *$

if DESC_MACRORAMO $=$ 'Alimentos' and PLANO $=5$ and VALOR $>=221$ and VALOR $<247$ then PID $=457$; else */Alimentos_5_F03:221 $\mid-247{ }^{*}$

if DESC_MACRORAMO = 'Construção' and PLANO $=5$ and VALOR $>=292$ and VALOR $<510$ then PID $=458$; ;lse */Construção_5_F03:292|-510/*

if DESC_MACRORAMO $=$ 'Diversão' and PLANO $=5$ and VALOR $>=216$ and VALOR $<451$ then PID $=459$;

if DESC_MACRORAMO $=$ 'Farmácia' and PLANO $=5$ and VALOR $>=100$ and VALOR $<139$ then PID $=460$; else */Farmácia_5_F03:100|- $-139 /^{\star}$

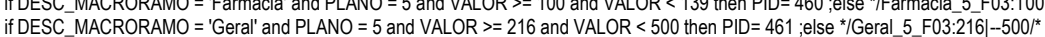

if DESC_MACRORAMO $=$ 'Joalherias' and PLANO $=5$ and VALOR $>=239$ and VALOR $<340$ then PID $=462$; else */Joalherias_5_F03:239|--340/*

if DESC_MACRORAMO $=$ 'Joalherias' and PLANO $=5$ and VALOR $>=239$ and VALOR $<340$ then PID $=462$; else */Joalherias_5_F03:239|--340
if DESC_MACRORAMO $=$ 'Livraria' and PLANO $=5$ and VALOR $>=163$ and VALOR $<274$ then PID $=463$;else */Livraria_5_F03:163|-274/*

if DESC_MACRORAMO $=$ 'Livraria' and PLANO $=5$ and VALOR $>=163$ and VALOR $<274$ then PID $=463$;else */Livraria_5_FO3:163|--274/ ${ }^{*}$
if DESC_MACRORAMO $=$ 'Lojas em Geral' and PLANO $=5$ and VALOR $>=136$ and VALOR $<223$ then PID $=464 ;$;else */Lojas em Geral_5 F03:136|-223/ ${ }^{*}$

if DESC_MACRORAMO $=$ 'Lojas em Geral' and PLANO $=5$ and VALOR $>=136$ and VALOR $<223$ then PID $=464$; else */Lojas em Geral_5_F
if DESC_MACRORAMO $=$ 'Móveis' and PLANO $=5$ and VALOR $>=253$ and VALOR $<425$ then PID $=465$;else */Móveis 5 F03:253|--425/*

if DESC_MACRORAMO $=$ 'Móveis' and PLANO $=5$ and VALOR $>253$ and VALOR $<425$ then PID $=465$; else */Móveis_5_F03:253|--425/*
if DESC MACRORAMO $=$ 'Perfumaria' and PLANO $=5$ and VALOR $>=154$ and VALOR $<204$ then PID 466 ;else */Perfumaria 5 F03:154|--204/*

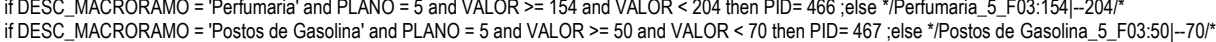

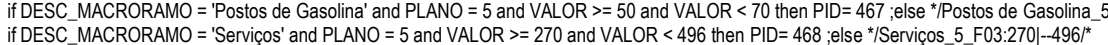

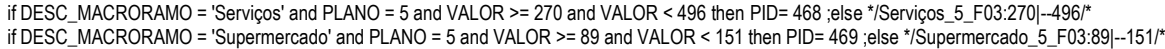

if DESC_MACRORAMO $=$ 'Telecomunicação / Eletrônicos' and PLANO $=5$ and VALOR $>=219$ and VALOR $<360$ then PID $=470$; ;else */Telecomunicação / Eletrônicos_5_F03:219|--360/*

if DESC_MACRORAMO = 'Vestuário' and PLANO $=5$ and VALOR $>=139$ and VALOR $<209$ then PID $=471$;else *Nestuário_5_F03:139|--209/*

if DESC_MACRORAMO $=$ 'Veiculos' and PLANO $=5$ and VALOR $>=278$ and VALOR $<481$ then PID $=472$; else *Neículos_5_F03:278|--481/*

if DESC_MACRORAMO $=$ 'Viagens' and PLANO $=5$ and VALOR $>=308$ and VALOR $<494$ then PID $=473$; else * $/$ Viagens_5_F03:308|--494/*

if DESC_MACRORAMO = 'Alimentos' and PLANO $=6$ and VALOR $>=145$ and VALOR $<164$ then PID $=474$;else */Alimentos_6_F03:145|--164/ ${ }^{*}$

if DESC_MACRORAMO $=$ 'Construção' and PLANO $=6$ and VALOR $>=392$ and VALOR $<654$ then PID $=475$;else */Construção_6_F03:392|--654/ ${ }^{*}$

if DESC_MACRORAMO $=$ 'Diversão' and PLANO $=6$ and VALOR $>=340$ and VALOR $<550$ then PID $=476$; else */Diversão_6_F03:340|--550/*
if DESC_MACRORAMO $=$ 'Farmácia' and PLANO $=6$ and VALOR $>=67$ and VALOR $<119$ then PID $=477$;else */Farmácia_6_F03:67|-119/*

if DESC_MACRORAMO = 'Geral' and PLANO $=6$ and VALOR $>=250$ and VALOR $<509$ then PID $=478$;else */Geral_6_F03:250|-509/*

if DESC_MACRORAMO $=$ 'Joalherias' and PLANO $=6$ and VALOR $>=298$ and VALOR $<415$ then PID $=479$;else */Joalherias_6_F03:298|--415/*

if DESC_MACRORAMO = 'Livraria' and PLANO $=6$ and VALOR $>=214$ and VALOR $<380$ then PID $=480$; ;

if DESC_MACRORAMO $=$ 'Lojas em Geral' and PLANO $=6$ and VALOR $>=179$ and VALOR $<317$ then PID $=481$; else */Lojas em Geral_6_F03:179|--317/

if DESC_MACRORAMO $=$ 'Lojas em Geral' and PLANO $=6$ and VALOR $>=179$ and VALOR $<317$ then PID $=481$; else *Lojas em Geral_6
if DESC_MACRORAMO $=$ 'Móveis' and PLANO $=6$ and VALOR $>=322$ and VALOR $<492$ then PID $=482$;else */Móveis_6_Fo3:322|--492/

if DESC_MACRORAMO $=$ 'Móveis' and PLANO $=6$ and VALOR $>=322$ and VALOR $<492$ then PID $=482$;else */Móveis_6_F03:322|--492/ ${ }^{*}$
if DESC_MACRORAMO $=$ 'Perfumaria' and PLANO $=6$ and VALOR $>=212$ and VALOR $<300$ then PID $=483 ;$;else $*$ Perfumaria_6_F03:212|--300/*

if DESC_MACRORAMO $=$ 'Perfumaria' and PLANO $=6$ and VALOR $>=212$ and VALOR $<300$ then PID $=483$;else */Perfumaria_6_F03:212|-300/ ${ }^{*}$
if DESC_MACRORAMO $=$ 'Postos de Gasolina' and PLANO $=6$ and VALOR $>=358$ and VALOR $<399$ then PID $=484$;else */Postos de Gasolina_6_F03:358|-399/*

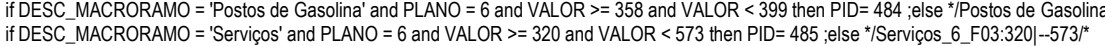

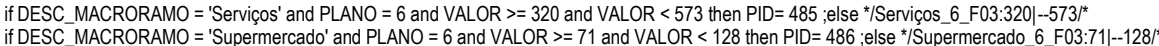

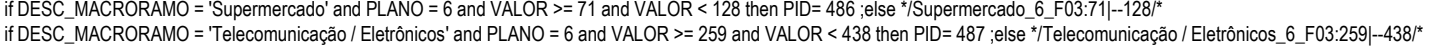

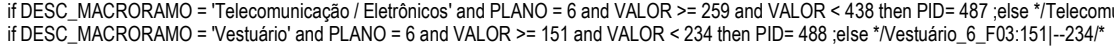

if DESC_MACRORAMO $=$ 'Veículos' and PLANO $=6$ and VALOR $>=330$ and VALOR $<588$ then PID $=489$;

if DESC_MACRORAMO $=$ 'Viagens' and PLANO $=6$ and VALOR $>=517$ and VALOR $<728$ then PID $=490$;else */Viagens_6_F03:517|--728/*

if DESC_MACRORAMO = 'Construção' and PLANO $=7$ and VALOR $>=347$ and VALOR $<591$ then PID $=491$; else $*$ Construção_7_FO3:347|--591/

if DESC_MACRORAMO = 'Diversão' and PLANO $=7$ and VALOR $>=620$ and VALOR < 693 then PID $=492$; else */Diversão_7_F03:620|--693/* 
if DESC_MACRORAMO = 'Farmácia' and PLANO $=7$ and VALOR $>=78$ and VALOR $<119$ then PID $=493$;else */Farmácia_7_F03:78|-119/* if DESC_MACRORAMO = 'Geral' and PLANO $=7$ and VALOR $>=220$ and VALOR $<499$ then PID $=494$;else */Geral_7_F03:220|--499/* if DESC_MACRORAMO $=$ 'Joalherias' and PLANO $=7$ and VALOR $>=307$ and VALOR $<452$ then PID $=495$;else */Joalherias_7_F03:307|--452/* if DESC_MACRORAMO = 'Livraria' and PLANO $=7$ and VALOR $>=209$ and VALOR $<410$ then PID $=496$; else */Livraria_7_F03:209|--410/* if DESC_MACRORAMO = 'Lojas em Geral' and PLANO $=7$ and VALOR $>=187$ and VALOR $<349$ then PID $=497$;else */Lojas em Geral_7_F03:187|--349/* if DESC_MACRORAMO = 'Lojas em Geral and PLANO $=7$ and VALOR $>=187$ and VALOR $<349$ then PID $=497$; else * $*$ Lojas em Geral_7_ if DESC_MACRORAMO $=$ 'Móveis' and PLANO $=7$ and VALOR $>=374$ and VALOR $<677$ then PID $=498$;else */Móveis_7_F03:374|--677/ $\left.\right|^{*}$
if DESC MACRORAMO $=$ 'Perfumaria' and PLANO $=7$ and VALOR $>=135$ and VALOR $<169$ then PID $=499$;else */Perfumaria 7 F03:135|--169/*

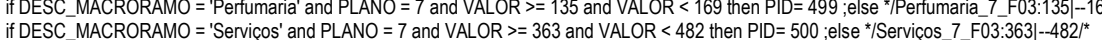
if DESC_MACRORAMO $=$ 'Serviços' and PLANO $=7$ and VALOR $>=363$ and VALOR $<482$ then PID $=500$;else */ServiçoS_7_F03:363|--482/*
if DESC MACRORAMO $=$ 'Supermercado' and PLANO $=7$ and VALOR $>=114$ and VALOR $<297$ then PID $=501 ;$ else */Supermercado_7_F03:114|-297/*

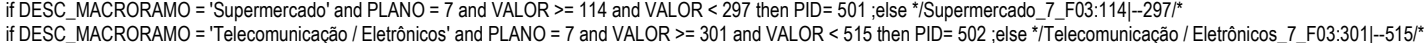

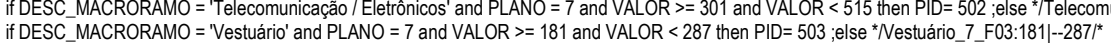
if DESC_MACRORAMO = 'Vestuário' and PLANO $=7$ and VALOR $>=181$ and VALOR $<287$ then PID $=503$;else * Nestuário_7_F03:181|--287/" if DESC_MACRORAMO $=$ 'Veículos' and PLANO $=7$ and VALOR $>=259$ and VALOR $<439$ then PID $=504$;else */Veículos_7_F03:259|--439/*
if DESC_MACRORAMO $=$ 'Viagens' and PLANO $=7$ and VALOR $>=325$ and VALOR $<700$ then PID $=505$;else */Viagens_7_F03:325|--700/* if DESC_MACRORAMO $=$ 'Construção' and PLANO $=8$ and VALOR $>=551$ and VALOR $<903$ then PID $=506$;else */Construção_8_F03:551|--903/* if DESC_MACRORAMO = 'Diversão' and PLANO $=8$ and VALOR $>=560$ and VALOR $<560$ then PID= 507 ;else */Diversão_8_F03:560|--560/* if DESC_MACRORAMO $=$ 'Farmácia' and PLANO $=8$ and VALOR $>=93$ and VALOR $<164$ then PID $=508$;else */Farmácia_8_F03:93|--164/" if DESC_MACRORAMO = 'Geral' and PLANO $=8$ and VALOR $>=262$ and VALOR $<563$ then PID $=509$;else */Geral____F03:262|--563/* if DESC_MACRORAMO $=$ 'Joalherias' and PLANO $=8$ and VALOR $>=440$ and VALOR $<640$ then PID $=510$; else */Joalherias_8_F03:440|--640/* if DESC_MACRORAMO = 'Livraria' and PLANO $=8$ and VALOR $>=206$ and VALOR $<425$ then PID = 511; ;else */Livraria_8_F03:206|--425/* if DESC_MACRORAMO = 'Lojas em Geral' and PLANO = 8 and VALOR $>=250$ and VALOR $<399$ then PID= 512 ;else */Lojas em Geral_8_F03:250|-399/* if DESC_MACRORAMO = 'Móveis' and PLANO $=8$ and VALOR $>=424$ and VALOR $<699$ then PID $=513$;else */Móveis_8_F03:424|--699/* if DESC_MACRORAMO $=$ 'Perfumaria' and PLANO $=8$ and VALOR $>=239$ and VALOR $<300$ then PID $=514$; else */Perfumaria_8_F03:239|--300/* if DESC_MACRORAMO = 'Serviços' and PLANO = 8 and VALOR $>=457$ and VALOR $<1000$ then PID $=515$;else */Serviços 8 F F $03: 457 \mid-1000$ /* if DESC_MACRORAMO = 'Supermercado' and PLANO $=8$ and VALOR $>=158$ and VALOR $<337$ then PID $=516$; ;lse */Supermercado_8_F03:158|--337/* if DESC_MACRORAMO = 'Telecomunicação / Eletrônicos' and PLANO $=8$ and VALOR $>=336$ and VALOR $<584$ then PID $=517$; else */Telecomunicação / Eletrônicos_8_F03:336|--584/* if DESC MACRORAMO $=$ 'Vestuário' and PLANO $=8$ and VALOR $>=174$ and VALOR $<271$ then PID $=518$;else * $*$ Vestuário 8 FO3:174|--271/* if DESCMACRORAMO = 'Vestuanto' and PLANO $=8$ and VALOR $>=174$ and VALOR $<271$ then PID $=518$,else "Nestuario_8_F03:174|--271/* if DESC_MACRORAMO $=$ 'Veículos' and PLANO $=8$ and VALOR $>=499$ and VALOR $<785$ then PID $=519$; else * $/$ Veículos_8_F03:499|--785/* if DESC_MACRORAMO $=$ 'Viagens' and PLANO $=8$ and VALOR $>=658$ and VALOR $<1206$ then PID $=520$;else *Niagens_8_F03:658|--1206/*
if DESC_MACRORAMO $=$ 'Construção' and PLANO $=9$ and VALOR $>=695$ and VALOR $<973$ then PID $=521$;else */Construção_9_F03:695|--973/ if DESC_MACRORAMO $=$ 'Construçãa' and PLANO $=9$ and VALOR $>=695$ and VALOR $<973$ then PID $=521$; else */Construção_9_FO3:695|--973"
if DESC_MACRORAMO $=$ 'Diversão' and PLANO $=9$ and VALOR $>=337$ and VALOR $<337$ then PID $=522$;else */Diversão_9_F03:337|--337/*

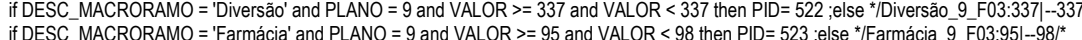
if DESC_MACRORAMO $=$ 'Farmácia' and PLANO $=9$ and VALOR $>=95$ and VALOR $<98$ then PID $=523$; else */Farmácia_ 9 _FO3:95|--
if DESC_MACRORAMO $=$ 'Geral' and PLANO $=9$ and VALOR $>=97$ and VALOR $<180$ then PID $=524$;else $/$ Geral 9 F03:97|--180/* if DESC_MACRORAMO $=$ 'Geral' and PLANO $=9$ and VALOR $>=97$ and VALOR $<180$ then PID $=524$;else */Geral_9_FO3:97|--180/*
if DESC_MACRORAMO $=$ 'Joalherias' and PLANO $=9$ and VALOR $>=208$ and VALOR $<290$ then PID $=525$;else */Joalherias_9_F03:208|--290/*

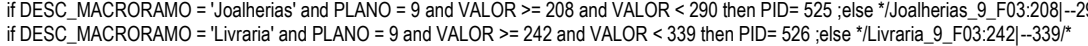
if DESC_MACRORAMO $=$ 'Lojas em Geral' and PLANO $=9$ and VALOR $>=219$ and VALOR $<364$ then PID $=527$; else */Lojas em Geral_9_F03:219|--364/* if DESC_MACRORAMO = 'Móveis' and PLANO $=9$ and VALOR $>=556$ and VALOR $<854$ then PID $=528$;else */Móveis_9_F03:556|--854/* if DESC_MACRORAMO $=$ 'Serviços' and PLANO $=9$ and VALOR $>292$ and VALOR $<422$ then PID $=529$; if DESC_MACRORAMO $=$ 'Supermercado' and PLANO $=9$ and VALOR $>=149$ and VALOR $<358$ then PID $=530 ;$;else $* /$ Supermercado 9 F F 03:149|-358/* if DESC_MACRORAMO = 'Telecomunicação / Eletrônicos' and PLANO $=9$ and VALOR $>=282$ and VALOR $<482$ then PID $=531$;else */Telecomunicação / Eletrônicos_9_F03:282|--482/ ${ }^{*}$ if DESC_MACRORAMO = 'Vestuário' and PLANO $=9$ and VALOR $>=99$ and VALOR $<137$ then PID $=532$; else *Nestuário_9_F03:99|--137/ ${ }^{*}$ if DESC_MACRORAMO $=$ 'Veículos' and PLANO $=9$ and VALOR $>=480$ and VALOR $<480$ then PID $=533$;else *Veículos 9 _F03:480|--480/* if DESC_MACRORAMO $=$ 'Viagens' and PLANO $=9$ and VALOR $>=520$ and VALOR $<649$ then PID $=534$;else *Niagens_ 9 F03:520|- $-649{ }^{*}$ if DESC_MACRORAMO = 'Alimentos' and PLANO $=10$ and VALOR > 108 and VALOR $<108$ then PID = 535 ;else */Alimentos_10_F03:108|--108/* if DESC_MACRORAMO = 'Construção' and PLANO = 10 and VALOR >= 600 and VALOR $<1036$ then PID = 536 ;else */Construção_10_F03:600|--1036/* if DESC_MACRORAMO = 'Diversão' and PLANO $=10$ and VALOR $>=300$ and VALOR $<540$ then PID $=537$; else */Diversão_10_F03:300|-540/* if DESC_MACRORAMO = 'Farmácia' and PLANO $=10$ and VALOR $>=109$ and VALOR $<160$ then PID= 538 ;else */Farmácia_10_F03:109|-160/* if DESC_MACRORAMO $=$ 'Geral' and PLANO $=10$ and VALOR $>=421$ and VALOR $<800$ then PID $=539$; if DESC_MACRORAMO $=$ 'Joalherias' and PLANO $=10$ and VALOR $>=450$ and VALOR $<650$ then PID $=540$;else */Joalherias_10_F03:450|--650/* if DESC_MACRORAMO $=$ 'Joalherias' and PLANO $=10$ and VALOR $>=450$ and VALOR $<650$ then PID $=540$;else $* /$ Joalherias_10_F03:450|---
if DESC MACRORAMO $=$ 'Livraria' and PLANO $=10$ and VALOR $>=295$ and VALOR $<445$ then PID $=541$;else */Livraria_10_F03:295|--445/* if DESC_MACRORAMO $=$ 'Livraria' and PLANO $=10$ and VALOR $>=295$ and VALOR $<445$ then PID= 541 ;else */Livraria_10_F03:295|--445/*
if DESC_MACRORAMO $=$ 'Lojas em Geral' and PLANO $=10$ and VALOR $>=259$ and VALOR $<440$ then PID $=542$;else */Lojas em Geral_10_F03:259|--440/* if DESC_MACRORAMO $=$ 'Lojas em Geral' and PLANO $=10$ and VALOR $>=259$ and VALOR $<440$ then PID $=542$;else */Lojas em Geral_10_F03:2
if DESC_MACRORAMO $=$ 'Móveis' and PLANO $=10$ and VALOR $>=499$ and VALOR $<809$ then PID $=543$;else */Móveis 10 F03:499|--809/* if DESC_MACRORAMO $=$ 'Móveis' and PLANO $=10$ and VALOR $>=499$ and VALOR $<809$ then PID $=543$;else $* /$ Móveis_10_F03:499|--809/* ${ }^{*}$
if DESC_MACRORAMO $=$ 'Perfumaria' and PLANO $=10$ and VALOR $>=319$ and VALOR $<557$ then PID $=544$;else $* /$ Perfumaria_10_F03:319|--557/

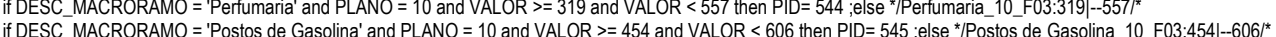
if DESC_MACRORAMO $=$ 'Postos de Gasolina' and PLANO $=10$ and VALOR $>=454$ and VALOR $<606$ then PID $=545$;else */Postos de Gasolina
if DESC_MACRORAMO = 'Servicos' and PLANO $=10$ and VALOR $>=478$ and VALOR $<672$ then PID $=546$;else */Servicos 10 F03:478|--672/* if DESC_MACRORAMO $=$ 'Serviços' and PLANO $=10$ and VALOR $>=478$ and VALOR $<672$ then PID $=546$;else */Serviços_10_F03:478|--672/* ${ }^{*}$ if DESC_MACRORAMO $=$ 'Supermercado' and PLANO $=10$ and VALOR $>=198$ and VALOR $<351$ then PID $=547$;else */Supermercado_10_F03:198|--351/ ${ }^{*}$
if DESC_MACRORAMO = 'Telecomunicação / Eletrônicos' and PLANO $=10$ and VALOR $>=350$ and VALOR $<603$ then PID= 548 ;else */Telecomunicação / Eletrônicos_10_F03:350|--603/* if DESC_MACRORAMO $=$ 'Telecomunicação / Eletrônicos' and PLANO $=10$ and VALOR $>=350$ and VALOR $<603$ then PID $=548 ;$;else $* / T e l e c o m$
if DESC_MACRORAMO $=$ 'Vestuário' and PLANO $=10$ and VALOR $>=199$ and VALOR $<303$ then PID $=549 ;$;else * $*$ Vestuário_10_F03:199|--303/ if DESC_MACRORAMO $=$ 'Veículos' and PLANO $=10$ and VALOR $>=418$ and VALOR $<730$ then PID $=550$;else ${ }^{*}$ Neículos_10_F03:418|--730/* if DESC_MACRORAMO $=$ 'Viagens' and PLANO $=10$ and VALOR $>=558$ and VALOR $<1139$ then PID $=551 ;$;else * ${ }^{*}$ Viagens_10_F03:558|--1139/ if DESC MACRORAMO $=$ 'Geral' and PLANO $=11$ and VALOR $>=220$ and VALOR $<220$ then PID $=552$; else */Geral 11 F03:220|-220/* if DESC_MACRORAMO = 'Livraria' and PLANO $=11$ and VALOR $>=296$ and VALOR $<399$ then PID= 553 ;else */Livraria_11_F03:296|--399/* if DESC_MACRORAMO = 'Lojas em Geral' and PLANO $=11$ and VALOR $>=465$ and VALOR $<862$ then PID $=554$;else */Lojas em Geral_11_F03:465|--862/* if DESC MACRORAMO = 'Móveis' and PLANO = 11 and VALOR $>=530$ and VALOR $<665$ then PID= 555 ;else */Móveis 11 F03:530|- $665{ }^{*}$ if DESC_MACRORAMO = 'Supermercado' and PLANO $=11$ and VALOR $>=205$ and VALOR $<475$ then PID $=556$;else */Supermercado 11 F03:205|--475/* if DESC_MACRORAMO = 'Telecomunicação / Eletrônicos' and PLANO $=11$ and VALOR > 606 and VALOR $<887$ then PID $=557$; :else */Telecomunicação / Eletrônicos 11 F03:606/--887/ ${ }^{*}$ if DESC_MACRORAMO = 'Vestuário' and PLANO $=11$ and VALOR $>=223$ and VALOR $<227$ then PID $=558$;else *Nestuário_11_F03:223|-227/* if DESC_MACRORAMO = 'Construção' and PLANO $=12$ and VALOR $>=684$ and VALOR $<1100$ then PID $=559$;else */Construção_12_F03:684|--1100/* if DESC MACRORAMO = 'Farmácia' and PLANO $=12$ and VALOR $>=261$ and VALOR $<443$ then $\mathrm{PID}=560$-else */Farmácia 12 F03-261/-443/* if DESC_MACRORAMO $=$ 'Geral' and PLANO $=12$ and VALOR $>=509$ and VALOR $<717$ then PID $=561$; else * */Geral 12 F $03: 509 \mid--7177^{*}$ if DESC_MACRORAMO $=$ 'Joalherias' and PLANO $=12$ and VALOR $>=502$ and VALOR $<798$ then PID $=562$; else */Joalherias 12 F03:502|--798/* if DESC_MACRORAMO $=$ 'Joalherias' and PLANO $=12$ and VALOR $>=502$ and VALOR $<798$ then PID $=562$;else */Joalherias_12_F03:502|---7
if DESC_MACRORAMO $=$ 'Livraria' and PLANO $=12$ and VALOR $>=249$ and VALOR $<510$ then PID $=563$; else */Livraria_12_F03:249|--510/* if DESC_MACRORAMO $=$ 'Livraria' and PLANO $=12$ and VALOR $>=249$ and VALOR $<510$ then PID $=563$;else */Livraria_12_F03:249|--510/*
if DESC_MACRORAMO $=$ 'Lojas em Geral' and PLANO $=12$ and VALOR $>=442$ and VALOR $<871$ then PID $=564$;else */Lojas em Geral_12_F03:442|--871/*

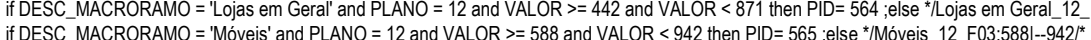
if DESC_MACRORAMO $=$ 'Móveis' and PLANO $=12$ and VALOR $>=588$ and VALOR $<942$ then PID $=565$;else */Móveis_12_F03:588|--942/*
if DESC_MACRORAMO $=$ 'Perfumaria' and PLANO $=12$ and VALOR $>=295$ and VALOR $<495$ then PID $=566$; else */Perfumaria_12 F03:295|--495/*

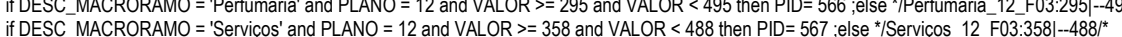
if DESC_MACRORAMO = 'Serviços' and PLANO $=12$ and VALOR $>=358$ and VALOR $<488$ then PID $=567$; else */Serviços_12_F03:358|--488/* ${ }^{*}$

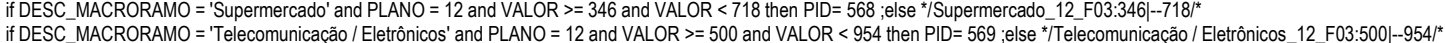
if DESC_MACRORAMO $=$ 'Telecomunicação / Eletrônicos' and PLANO $=12$ and VALOR $>=500$ and VALOR $<954$ then PID $=569$;else */Telecom
if DESC_MACRORAMO $=$ 'Vestuário' and PLANO $=12$ and VALOR $>=259$ and VALOR $<379$ then PID $=570$;else *Nestuário 12 F03:259|--379/* if DESC MACRORAMO $=$ 'Veiculos' and PLANO $=12$ and VALOR $>=430$ and VALOR $<752$ then PID $=571$; else * Neículos 12 F03:430|--752/* if DESC_MACRORAMO = 'Viagens' and PLANO $=12$ and VALOR $>=589$ and VALOR $<595$ then PID $=572$; else *Niagens_12_F03:589|--595/* if DESC_MACRORAMO = 'Supermercado' and PLANO $=13$ and VALOR $>=787$ and VALOR $<1398$ then PID $=573$;else */Supermercado_13_F03:787|-1398/ if DESC_MACRORAMO = 'Lojas em Geral' and PLANO $=14$ and VALOR $>=577$ and VALOR $<577$ then PID $=574$;else */Lojas em Geral_14_F03:577|--577/ if DESC_MACRORAMO = 'Supermercado' and PLANO = 14 and VALOR $>=121$ and VALOR $<202$ then PID $=575$;else */Supermercado_14_F03:121|--202/* if DESC_MACRORAMO = 'Construção' and PLANO $=15$ and VALOR $>=228$ and VALOR $<228$ then PID= 576 ;else */Construção 15 F03:228|--228/* if DESC MACRORAMO = 'Livraria' and PLANO $=15$ and VALOR $>=910$ and VALOR $<910$ then PID $=577$; else */Livraria 15 F03:910|--910/* if DESC_MACRORAMO = 'Lojas em Geral' and PLANO $=15$ and VALOR $>=178$ and VALOR $<1103$ then PID $=578$;else ${ }^{*} /$ Lojas em Geral_15_F03:178|--1103/* if DESC MACRORAMO = 'Supermercado' and PLANO $=15$ and VALOR $>=548$ and VALOR $<1197$ then PID $=579$;else */Supermercado 15 F03:548|-1197/* if DESC_MACRORAMO = 'Telecomunicação / Eletrônicos' and PLANO = 15 and VALOR >= 1415 and VALOR < 1415 then PID= 580 ;else */Telecomunicação / Eletrônicos_15_F03:1415|--

if DESC MACRORAMO = 'Supermercado' and PLANO $=18$ and VALOR $>=762$ and VALOR $<1298$ then PID $=581$;else */Supermercado 18 F03:762|-1298*

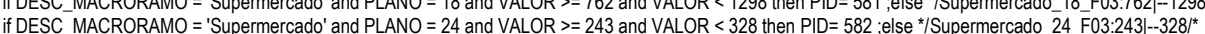
if DESC_MACRORAMO $=$ 'Supermercado' and PLANO $=24$ and VALOR $>=243$ and VALOR $<328$ then PID $=582$; ;else */Supermercado_24_*
if DESC_MACRORAMO $=$ 'Alimentos' and PLANO $=1$ and VALOR $>=39$ and VALOR $<82$ then PID $=583$;else */Alimentos_1_F04:39|--82 ${ }^{*}$ if DESC_MACRORAMO $=$ 'Alimentos' and PLANO $=1$ and VALOR $>=39$ and VALOR $<82$ then PID $=583$;else */Alimentos_1_F04:39|--82 ${ }^{*}$
if DESC_MACRORAMO $=$ 'Construção' and PLANO $=1$ and VALOR $>=47$ and VALOR $<102$ then PID $=584$;else */Construçâa__1_F04:47|--102/ if DESC_MACRORAMO $=$ 'Construção' and PLANO $=1$ and VALOR $>=47$ and VALOR $<102$ then PID $=584$;else */Construção_1_F04:47/|-
if DESC_MACRORAMO = 'Diversão' and PLANO $=1$ and VALOR $>=31$ and VALOR $<45$ then PID $=585$;else */Diversão_1_F04:31|-45/* if DESC_MACRORAMO $=$ 'Diversão' and PLANO $=1$ and VALOR $>=31$ and VALOR $<45$ then PID $=585$;else */Diversão_1_F04:31|--45/"
if DESC MACRORAMO $=$ 'Farmácia' and PLANO $=1$ and VALOR $>=27$ and VALOR $<44$ then PID $=586$;else */Farmácia 1 FO4:27|--44/ if DESC_MACRORAMO $=$ 'Farmácia' and PLANO $=1$ and VALOR $>=27$ and VALOR $<44$ then PID $=586$;else */Farmácia_1_F04:27-
if DESC_MACRORAMO $=$ 'Geral' and PLANO $=1$ and VALOR $>=38$ and VALOR $<83$ then PID $=587$;else */Geral_1_F04:38|--83/* if DESC_MACRORAMO = 'Joalherias' and PLANO $=1$ and VALOR $>=30$ and VALOR $<68$ then PID $=588$;else */Joalherias_1_F04:30|-68/* $\mathrm{PID}=9999 ; * /$ Outros $^{*}$ 


\section{Apêndice 2: Regras utilizadas na segmentação}

\section{Regras da ACC}

\begin{tabular}{|c|c|c|c|c|c|c|}
\hline $\begin{array}{l}\text { ID da } \\
\text { Regra }\end{array}$ & Consequente & Antecedente & Lift & Support Lift & Confidence & Support \\
\hline 1 & Viagens_1 & Viagens & 43,97 & $-0,34$ & 66,47 & 1,51 \\
\hline 16 & Livraria_1 & Livraria & 20,67 & $-0,44$ & 55,86 & 2,70 \\
\hline 17 & Joalherias_1 & Joalherias & 20,24 & $-0,62$ & 37,75 & 1,87 \\
\hline 40 & Servicos_1_F03:30|--50 & Servicos_1 & 15,95 & 0,18 & 29,61 & 1,86 \\
\hline 47 & Perfumaria_3 & Perfumaria & 14,39 & $-0,46$ & 18,03 & 1,25 \\
\hline 52 & Veiculos_4 & Veiculos & 12,21 & $-0,51$ & 12,27 & 1,00 \\
\hline 53 & Veiculos_3 & Veiculos & 12,21 & $-0,02$ & 24,59 & 2,01 \\
\hline 54 & Veiculos_1 & Veiculos & 12,21 & 0,82 & 45,44 & 3,72 \\
\hline 55 & Veiculos_2 & Veiculos & 12,21 & $-0,01$ & 24,74 & 2,03 \\
\hline 62 & Servicos_1 & Servicos & 11,07 & 1,08 & 69,39 & 6,27 \\
\hline 74 & Alimentos_1_F03:21|--39 & Alimentos_1 & 9,53 & 0,40 & 34,93 & 3,66 \\
\hline 78 & Alimentos_1 & Alimentos & 9,23 & $-0,03$ & 96,81 & 10,49 \\
\hline 93 & Diversao_1_F01:0|--12 & Diversao_1 & 8,19 & 0,40 & 28,07 & 3,43 \\
\hline 96 & Diversao_1 & Diversao & 8,00 & $-0,02$ & 97,69 & 12,21 \\
\hline 97 & $\begin{array}{l}\text { Postos de Gasolina_1_F02:18|--30 \& } \\
\text { Supermercado }\end{array}$ & Postos de Gasolina_1_F01:0|--18 & 5,29 & 0,07 & 20,20 & 1,14 \\
\hline 99 & $\begin{array}{l}\text { Postos de Gasolina_1_F02:18|--30 \& } \\
\text { Supermercado_1 }\end{array}$ & Postos de Gasolina_1_F01:0|--18 & 5,29 & 0,13 & 18,29 & 1,03 \\
\hline 102 & Postos de Gasolina_1_F02:18|--30 & $\begin{array}{l}\text { Postos de Gasolina_1_F01:0|--18 \& } \\
\text { Supermercado }\end{array}$ & 5,22 & 0,07 & 34,89 & 1,14 \\
\hline 103 & Postos de Gasolina_1_F02:18|--30 & $\begin{array}{l}\text { Postos de Gasolina_1_F01:0|--18 \& } \\
\text { Supermercado_1 }\end{array}$ & 5,20 & 0,13 & 34,77 & 1,03 \\
\hline 106 & Postos de Gasolina_1_F02:18|--30 & Postos de Gasolina_1_F01:0|--18 & 5,10 & 0,00 & 34,08 & 1,92 \\
\hline 108 & Postos de Gasolina_1_F02:18|--30 & Postos de Gasolina_1 & 5,09 & 1,04 & 33,98 & 6,68 \\
\hline 122 & Postos de Gasolina_1 & Postos de Gasolina & 4,94 & $-0,03$ & 97,09 & 19,66 \\
\hline 135 & Postos de Gasolina_1_F02:18|--30 & $\begin{array}{l}\text { Postos de Gasolina_1_F03:30|--50 \& } \\
\text { Supermercado_1 }\end{array}$ & 4,34 & $-0,08$ & 29,01 & 1,06 \\
\hline 142 & $\begin{array}{l}\text { Postos de Gasolina_1_F02:18|--30 \& } \\
\text { Supermercado_1 }\end{array}$ & Postos de Gasolina_1_F03:30|--50 & 4,30 & $-0,08$ & 14,88 & 1,06 \\
\hline 147 & Postos de Gasolina_1_F02:18|--30 & $\begin{array}{l}\text { Postos de Gasolina_1_F03:30|--50 \& } \\
\text { Supermercado }\end{array}$ & 4,29 & $-0,14$ & 28,66 & 1,16 \\
\hline 149 & $\begin{array}{l}\text { Postos de Gasolina_1_F02:18|--30 \& } \\
\text { Supermercado }\end{array}$ & Postos de Gasolina_1_F03:30|--50 & 4,25 & $-0,14$ & 16,23 & 1,16 \\
\hline 165 & Postos de Gasolina_1_F02:18|--30 & Postos de Gasolina_1_F03:30|--50 & 4,02 & $-0,21$ & 26,85 & 1,92 \\
\hline 169 & $\begin{array}{l}\text { Supermercado_1_F01:0|--13 \& Postos de } \\
\text { Gasolina }\end{array}$ & Supermercado_1_F02:13|--28 \& Farmacia & 3,96 & 0,92 & 15,02 & 1,01 \\
\hline 174 & Diversao_1 \& Vestuario & Farmacia \& Lojas em Geral \& Supermercado & 3,88 & 0,00 & 19,60 & 1,02 \\
\hline 179 & Diversao \& Vestuario & Farmacia \& Lojas em Geral \& Supermercado & 3,86 & 0,00 & 20,02 & 1,04 \\
\hline 187 & Diversao \& Lojas em Geral & Farmacia \& Supermercado \& Vestuario & 3,79 & 0,00 & 17,01 & 1,04 \\
\hline 190 & Diversao_1 \& Lojas em Geral & Farmacia \& Supermercado \& Vestuario & 3,78 & 0,00 & 16,64 & 1,02 \\
\hline 196 & $\begin{array}{l}\text { Postos de Gasolina_1 \& } \\
\text { Supermercado_1_F02:13|--28 }\end{array}$ & $\begin{array}{l}\text { Supermercado_1_F01:0|--13 \& } \\
\text { Supermercado_1_F03:28|--61 }\end{array}$ & 3,77 & 0,95 & 16,50 & 1,02 \\
\hline 199 & $\begin{array}{l}\text { Supermercado_1_F02:13|--28 \& Postos de } \\
\text { Gasolina }\end{array}$ & $\begin{array}{l}\text { Supermercado_1_F01:0|--13 \& } \\
\text { Supermercado__F03:28|--61 }\end{array}$ & 3,75 & 0,94 & 16,90 & 1,04 \\
\hline 201 & $\begin{array}{l}\text { Supermercado_1_F02:13|--28 \& Postos de } \\
\text { Gasolina }\end{array}$ & Supermercado_1_F01:0|--13 \& Farmacia & 3,74 & 0,92 & 16,85 & 1,01 \\
\hline 225 & Diversao_1 \& Supermercado \& Vestuario & Farmacia \& Lojas em Geral & 3,60 & 0,00 & 12,18 & 1,02 \\
\hline 228 & Diversao \& Supermercado \& Vestuario & Farmacia \& Lojas em Geral & 3,59 & 0,00 & 12,45 & 1,04 \\
\hline 237 & $\begin{array}{l}\text { Postos de Gasolina_1 \& } \\
\text { Supermercado_1_F01:0|--13 }\end{array}$ & $\begin{array}{l}\text { Supermercado_1_F02:13|--28 \& } \\
\text { Supermercado_1_F03:28|--61 }\end{array}$ & 3,57 & 0,95 & 13,14 & 1,02 \\
\hline
\end{tabular}




\begin{tabular}{|c|c|c|c|c|c|c|}
\hline 238 & $\begin{array}{l}\text { Supermercado_1_F01:0|--13 \& Postos de } \\
\text { Gasolina }\end{array}$ & $\begin{array}{l}\text { Supermercado_1_F02:13|--28 \& } \\
\text { Supermercado_1_F03:28|--61 }\end{array}$ & 3,54 & 0,94 & 13,45 & 1,04 \\
\hline 253 & Diversao \& Farmacia & Lojas em Geral \& Supermercado \& Vestuario & 3,47 & 0,00 & 18,14 & 1,04 \\
\hline 256 & Diversao_1 \& Farmacia & Lojas em Geral \& Supermercado \& Vestuario & 3,47 & 0,00 & 17,76 & 1,02 \\
\hline 267 & Diversao_1 \& Vestuario & Farmacia_1 \& Lojas em Geral & 3,40 & 0,09 & 17,22 & 1,08 \\
\hline 274 & Diversao \& Vestuario & Farmacia_1 \& Lojas em Geral & 3,39 & 0,09 & 17,59 & 1,10 \\
\hline 275 & Diversao \& Farmacia & Postos de Gasolina_1 \& Vestuario & 3,38 & 0,00 & 17,69 & 1,00 \\
\hline 276 & Postos de Gasolina_1 \& Vestuario & Diversao \& Farmacia & 3,38 & 0,00 & 19,16 & 1,00 \\
\hline 281 & Postos de Gasolina \& Vestuario & Diversao_1 \& Farmacia & 3,38 & 0,01 & 19,77 & 1,01 \\
\hline 282 & Diversao_1 \& Farmacia & Postos de Gasolina \& Vestuario & 3,38 & 0,01 & 17,31 & 1,01 \\
\hline 283 & $\begin{array}{l}\text { Diversao \& Lojas em Geral \& } \\
\text { Supermercado }\end{array}$ & Farmacia \& Vestuario & 3,38 & 0,00 & 10,27 & 1,04 \\
\hline 286 & $\begin{array}{l}\text { Diversao_1 \& Lojas em Geral \& } \\
\text { Supermercado }\end{array}$ & Farmacia \& Vestuario & 3,37 & 0,00 & 10,05 & 1,02 \\
\hline 288 & Diversao \& Farmacia & Postos de Gasolina \& Vestuario & 3,37 & 0,00 & 17,60 & 1,03 \\
\hline 289 & Postos de Gasolina \& Vestuario & Diversao \& Farmacia & 3,37 & 0,00 & 19,70 & 1,03 \\
\hline 303 & Diversao \& Lojas em Geral & Farmacia_1 \& Vestuario & 3,27 & 0,09 & 14,68 & 1,10 \\
\hline 307 & Diversao_1 \& Lojas em Geral & Farmacia_1 \& Vestuario & 3,27 & 0,09 & 14,36 & 1,08 \\
\hline 320 & Diversao_1 \& Vestuario & Farmacia \& Lojas em Geral & 3,17 & 0,00 & 16,05 & 1,35 \\
\hline 322 & Diversao_1 \& Supermercado_1 & Postos de Gasolina_1 \& Lojas em Geral & 3,17 & 0,11 & 22,23 & 1,09 \\
\hline 323 & Postos de Gasolina_1 \& Lojas em Geral & Diversao_1\& Supermercado_1 & 3,17 & 0,11 & 15,58 & 1,09 \\
\hline 324 & Diversao \& Vestuario & Farmacia \& Lojas em Geral & 3,17 & 0,00 & 16,42 & 1,38 \\
\hline 328 & Diversao_1 \& Supermercado_1 & Lojas em Geral \& Postos de Gasolina & 3,15 & 0,11 & 22,08 & 1,12 \\
\hline 329 & Lojas em Geral \& Postos de Gasolina & Diversao_1\& Supermercado_1 & 3,15 & 0,11 & 16,02 & 1,12 \\
\hline 330 & Postos de Gasolina_1 \& Lojas em Geral & Supermercado_1 \& Diversao & 3,15 & 0,10 & 15,46 & 1,10 \\
\hline 331 & Supermercado_1 \& Diversao & Postos de Gasolina_1 \& Lojas em Geral & 3,15 & 0,10 & 22,49 & 1,10 \\
\hline 332 & Diversao_1 \& Farmacia & Alimentos_1 \& Supermercado & 3,14 & 0,02 & 16,12 & 1,00 \\
\hline 333 & Alimentos_1\& Supermercado & Diversao_1 \& Farmacia & 3,14 & 0,02 & 19,60 & 1,00 \\
\hline 334 & $\begin{array}{l}\text { Diversao_1 \& Supermercado_1_F02:13|-- } \\
28\end{array}$ & Supermercado_1_F01:0|--13 & 3,14 & 0,91 & 10,79 & 1,70 \\
\hline 336 & Alimentos \& Supermercado & Diversao_1 \& Farmacia & 3,14 & 0,01 & 20,07 & 1,03 \\
\hline 337 & Diversao_1 \& Farmacia & Alimentos \& Supermercado & 3,14 & 0,01 & 16,07 & 1,03 \\
\hline 338 & Lojas em Geral \& Postos de Gasolina & Supermercado_1\& Diversao & 3,13 & 0,10 & 15,90 & 1,14 \\
\hline 339 & Supermercado_1 \& Diversao & Lojas em Geral \& Postos de Gasolina & 3,13 & 0,10 & 22,35 & 1,14 \\
\hline 341 & Diversao_1 \& Vestuario & Lojas em Geral_1\& Supermercado & 3,13 & 0,20 & 15,83 & 1,02 \\
\hline 345 & Supermercado_1_F02:13|--28 \& Diversao & Supermercado_1_F01:0|--13 & 3,12 & 0,90 & 10,93 & 1,73 \\
\hline 347 & Diversao \& Farmacia & Alimentos_1\& Supermercado & 3,12 & 0,01 & 16,31 & 1,02 \\
\hline 348 & Alimentos_1 \& Supermercado & Diversao \& Farmacia & 3,12 & 0,01 & 19,45 & 1,02 \\
\hline 355 & Alimentos \& Supermercado & Diversao \& Farmacia & 3,11 & 0,00 & 19,92 & 1,04 \\
\hline 356 & Diversao \& Farmacia & Alimentos \& Supermercado & 3,11 & 0,00 & 16,26 & 1,04 \\
\hline 360 & Diversao \& Vestuario & Lojas em Geral_1\& Supermercado & 3,10 & 0,19 & 16,08 & 1,04 \\
\hline 365 & Postos de Gasolina_1 \& Lojas em Geral & Diversao_1 \& Supermercado & 3,08 & 0,01 & 15,13 & 1,15 \\
\hline 366 & Diversao_1 \& Supermercado & Postos de Gasolina_1 \& Lojas em Geral & 3,08 & 0,01 & 23,50 & 1,15 \\
\hline 370 & Diversao \& Supermercado & Farmacia \& Lojas em Geral \& Vestuario & 3,07 & 0,00 & 23,89 & 1,04 \\
\hline 372 & Diversao_1 \& Supermercado & Lojas em Geral \& Postos de Gasolina & 3,07 & 0,01 & 23,40 & 1,19 \\
\hline 373 & Lojas em Geral \& Postos de Gasolina & Diversao_1 \& Supermercado & 3,07 & 0,01 & 15,59 & 1,19 \\
\hline 376 & Diversao_1 \& Supermercado & Farmacia \& Lojas em Geral \& Vestuario & 3,07 & 0,00 & 23,39 & 1,02 \\
\hline 378 & Diversao \& Supermercado & Postos de Gasolina_1 \& Lojas em Geral & 3,06 & 0,00 & 23,83 & 1,17 \\
\hline
\end{tabular}




\begin{tabular}{|c|c|c|c|c|c|c|}
\hline 379 & Postos de Gasolina_1 \& Lojas em Geral & Diversao \& Supermercado & 3,06 & 0,00 & 15,04 & 1,17 \\
\hline 382 & Diversao_1 & $\begin{array}{l}\text { Postos de Gasolina_1 \& Supermercado_1 \& } \\
\text { Lojas em Geral }\end{array}$ & 3,06 & 0,11 & 37,35 & 1,09 \\
\hline 385 & Lojas em Geral \& Postos de Gasolina & Diversao \& Supermercado & 3,05 & 0,00 & 15,50 & 1,21 \\
\hline 386 & Diversao \& Supermercado & Lojas em Geral \& Postos de Gasolina & 3,05 & 0,00 & 23,74 & 1,21 \\
\hline 390 & Diversao_1 & $\begin{array}{l}\text { Supermercado_1 \& Lojas em Geral \& Postos } \\
\text { de Gasolina }\end{array}$ & 3,04 & 0,11 & 37,15 & 1,12 \\
\hline 394 & Alimentos \& Vestuario & Farmacia \& Lojas em Geral & 3,04 & 0,00 & 12,14 & 1,02 \\
\hline 404 & Diversao & $\begin{array}{l}\text { Postos de Gasolina_1 \& Supermercado_1 \& } \\
\text { Lojas em Geral }\end{array}$ & 3,02 & 0,10 & 37,78 & 1,10 \\
\hline 407 & $\begin{array}{l}\text { Postos de Gasolina_1 \& } \\
\text { Supermercado_1_F03:28|--61 }\end{array}$ & $\begin{array}{l}\text { Supermercado_1_F01:0|--13 \& } \\
\text { Supermercado_1_F02:13|--28 }\end{array}$ & 3,02 & 0,95 & 13,57 & 1,02 \\
\hline 408 & Diversao_1 & Farmacia \& Postos de Gasolina \& Vestuario & 3,02 & 0,01 & 36,86 & 1,01 \\
\hline 410 & Diversao \& Lojas em Geral & Farmacia \& Vestuario & 3,02 & 0,00 & 13,55 & 1,38 \\
\hline 412 & $\begin{array}{l}\text { Supermercado_1_F03:28|--61 \& Postos de } \\
\text { Gasolina }\end{array}$ & $\begin{array}{l}\text { Supermercado_1_F01:0|--13 \& } \\
\text { Supermercado_1_F02:13|--28 }\end{array}$ & 3,01 & 0,94 & 13,89 & 1,04 \\
\hline 414 & Diversao_1\& Supermercado_1 & Postos de Gasolina_1 \& Vestuario & 3,01 & 0,10 & 21,11 & 1,19 \\
\hline 415 & Postos de Gasolina_1 \& Vestuario & Diversao_1 \& Supermercado_1 & 3,01 & 0,10 & 17,06 & 1,19 \\
\hline 416 & Diversao_1 \& Lojas em Geral & Farmacia \& Vestuario & 3,01 & 0,00 & 13,25 & 1,35 \\
\hline 420 & Diversao_1 & $\begin{array}{l}\text { Postos de Gasolina_1 \& Supermercado_1 \& } \\
\text { Vestuario }\end{array}$ & 3,01 & 0,10 & 36,75 & 1,19 \\
\hline 422 & Diversao & $\begin{array}{l}\text { Postos de Gasolina_1 \& Farmacia \& } \\
\text { Vestuario }\end{array}$ & 3,01 & 0,00 & 37,60 & 1,00 \\
\hline 423 & Diversao & $\begin{array}{l}\text { Supermercado_1 \& Lojas em Geral \& Postos } \\
\text { de Gasolina }\end{array}$ & 3,01 & 0,10 & 37,60 & 1,14 \\
\hline 428 & Diversao_1 & $\begin{array}{l}\text { Supermercado_1 \& Postos de Gasolina \& } \\
\text { Vestuario }\end{array}$ & 3,00 & 0,10 & 36,65 & 1,23 \\
\hline 429 & $\begin{array}{l}\text { Supermercado_1 \& Postos de Gasolina \& } \\
\text { Vestuario }\end{array}$ & Diversao_1 & 3,00 & 0,10 & 10,06 & 1,23 \\
\hline 430 & Supermercado_1\& Diversao & Postos de Gasolina_1 \& Vestuario & 3,00 & 0,09 & 21,41 & 1,21 \\
\hline 431 & Postos de Gasolina_1 \& Vestuario & Supermercado_1 \& Diversao & 3,00 & 0,09 & 16,97 & 1,21 \\
\hline 432 & Diversao & Farmacia \& Postos de Gasolina \& Vestuario & 3,00 & 0,00 & 37,47 & 1,03 \\
\hline 433 & Postos de Gasolina \& Vestuario & Diversao_1 \& Supermercado_1 & 3,00 & 0,10 & 17,54 & 1,23 \\
\hline 434 & Diversao_1\& Supermercado_1 & Postos de Gasolina \& Vestuario & 3,00 & 0,10 & 21,00 & 1,23 \\
\hline 435 & Diversao \& Farmacia & Lojas em Geral_1 \& Supermercado & 3,00 & 0,23 & 15,66 & 1,01 \\
\hline 437 & Diversao_1 & $\begin{array}{l}\text { Postos de Gasolina_1 \& Lojas em Geral \& } \\
\text { Supermercado }\end{array}$ & 2,99 & 0,01 & 36,50 & 1,15 \\
\hline 440 & Diversao & $\begin{array}{l}\text { Postos de Gasolina_1 \& Supermercado_1 \& } \\
\text { Vestuario }\end{array}$ & 2,98 & 0,09 & 37,29 & 1,21 \\
\hline 441 & Supermercado_1\& Diversao & Postos de Gasolina \& Vestuario & 2,98 & 0,09 & 21,29 & 1,25 \\
\hline 442 & Postos de Gasolina \& Vestuario & Supermercado_1\& Diversao & 2,98 & 0,09 & 17,45 & 1,25 \\
\hline 443 & Alimentos_1\& Farmacia & Diversao_1 \& Supermercado & 2,98 & 0,02 & 13,17 & 1,00 \\
\hline 444 & Diversao_1 \& Supermercado & Alimentos_1 \& Farmacia & 2,98 & 0,02 & 22,74 & 1,00 \\
\hline 445 & Postos de Gasolina_1 & Supermercado_1\&Veiculos_1 & 2,98 & 0,32 & 58,58 & 1,02 \\
\hline 447 & Diversao_1 \& Vestuario & Supermercado_1 \& Lojas em Geral & 2,98 & 0,09 & 15,07 & 1,62 \\
\hline 448 & Diversao_1\& Supermercado_1 & Farmacia_1 \& Postos de Gasolina_1 & 2,98 & 0,23 & 20,87 & 1,15 \\
\hline 449 & Farmacia_1 \& Postos de Gasolina_1 & Diversao_1\& Supermercado_1 & 2,98 & 0,23 & 16,36 & 1,15 \\
\hline 450 & Diversao_1 & $\begin{array}{l}\text { Lojas em Geral \& Postos de Gasolina \& } \\
\text { Supermercado }\end{array}$ & 2,98 & 0,01 & 36,35 & 1,19 \\
\hline 451 & Diversao & $\begin{array}{l}\text { Supermercado_1 \& Postos de Gasolina \& } \\
\text { Vestuario }\end{array}$ & 2,97 & 0,09 & 37,17 & 1,25 \\
\hline 452 & Postos de Gasolina & Supermercado_1 \& Veiculos_1 & 2,97 & 0,31 & 60,21 & 1,05 \\
\hline 456 & Farmacia_1 \& Postos de Gasolina & Diversao_1 \& Supermercado_1 & 2,97 & 0,23 & 16,72 & 1,17 \\
\hline 457 & Diversao_1\& Supermercado_1 & Farmacia_1 \& Postos de Gasolina & 2,97 & 0,23 & 20,80 & 1,17 \\
\hline 459 & Alimentos \& Farmacia & Diversao_1 \& Supermercado & 2,96 & 0,01 & 13,49 & 1,03 \\
\hline 460 & Diversao_1 \& Supermercado & Alimentos \& Farmacia & 2,96 & 0,01 & 22,61 & 1,03 \\
\hline 463 & Diversao & $\begin{array}{l}\text { Postos de Gasolina_1 \& Lojas em Geral \& } \\
\text { Supermercado }\end{array}$ & 2,96 & 0,00 & 37,02 & 1,17 \\
\hline
\end{tabular}




\begin{tabular}{|c|c|c|c|c|c|c|}
\hline 464 & Supermercado_1 \& Diversao & Farmacia_1 \& Postos de Gasolina_1 & 2,96 & 0,22 & 21,13 & 1,16 \\
\hline 465 & Farmacia_1 \& Postos de Gasolina_1 & Supermercado_1 \& Diversao & 2,96 & 0,22 & 16,25 & 1,16 \\
\hline 466 & Diversao \& Supermercado & Alimentos_1\& Farmacia & 2,96 & 0,01 & 23,01 & 1,02 \\
\hline 467 & Alimentos_1 \& Farmacia & Diversao \& Supermercado & 2,96 & 0,01 & 13,06 & 1,02 \\
\hline 468 & Postos de Gasolina_1 \& Vestuario & Diversao_1 \& Supermercado & 2,96 & 0,01 & 16,74 & 1,28 \\
\hline 469 & Diversao_1 \& Supermercado & Postos de Gasolina_1 \& Vestuario & 2,96 & 0,01 & 22,55 & 1,28 \\
\hline 471 & Diversao \& Vestuario & Supermercado_1 \& Lojas em Geral & 2,96 & 0,09 & 15,33 & 1,65 \\
\hline 473 & Diversao_1 \& Supermercado & Postos de Gasolina \& Vestuario & 2,95 & 0,01 & 22,51 & 1,32 \\
\hline 474 & Postos de Gasolina \& Vestuario & Diversao_1 \& Supermercado & 2,95 & 0,01 & 17,27 & 1,32 \\
\hline 479 & Diversao & $\begin{array}{l}\text { Lojas em Geral \& Postos de Gasolina \& } \\
\text { Supermercado }\end{array}$ & 2,95 & 0,00 & 36,87 & 1,21 \\
\hline 480 & Farmacia_1 \& Postos de Gasolina & Supermercado_1 \& Diversao & 2,95 & 0,21 & 16,60 & 1,19 \\
\hline 481 & Supermercado_1 \& Diversao & Farmacia_1 \& Postos de Gasolina & 2,95 & 0,21 & 21,06 & 1,19 \\
\hline 482 & $\begin{array}{l}\text { Postos de Gasolina_1 \& Supermercado \& } \\
\text { Vestuario }\end{array}$ & Diversao_1 & 2,95 & 0,01 & 10,45 & 1,28 \\
\hline 483 & Diversao_1 & $\begin{array}{l}\text { Postos de Gasolina_1 \& Supermercado \& } \\
\text { Vestuario }\end{array}$ & 2,95 & 0,01 & 36,01 & 1,28 \\
\hline 485 & Diversao_1 & $\begin{array}{l}\text { Postos de Gasolina \& Supermercado \& } \\
\text { Vestuario }\end{array}$ & 2,95 & 0,01 & 35,96 & 1,32 \\
\hline 486 & $\begin{array}{l}\text { Postos de Gasolina \& Supermercado \& } \\
\text { Vestuario }\end{array}$ & Diversao_1 & 2,95 & 0,01 & 10,79 & 1,32 \\
\hline 487 & Postos de Gasolina_1 \& Vestuario & Diversao \& Supermercado & 2,94 & 0,00 & 16,66 & 1,30 \\
\hline 488 & Diversao \& Supermercado & Postos de Gasolina_1 \& Vestuario & 2,94 & 0,00 & 22,91 & 1,30 \\
\hline 489 & Diversao \& Supermercado & Alimentos \& Farmacia & 2,94 & 0,00 & 22,88 & 1,04 \\
\hline 490 & Alimentos \& Farmacia & Diversao \& Supermercado & 2,94 & 0,00 & 13,38 & 1,04 \\
\hline 491 & Postos de Gasolina \& Vestuario & Diversao \& Supermercado & 2,94 & 0,00 & 17,19 & 1,34 \\
\hline 492 & Diversao \& Supermercado & Postos de Gasolina \& Vestuario & 2,94 & 0,00 & 22,86 & 1,34 \\
\hline 495 & Diversao_1 & $\begin{array}{l}\text { Farmacia \& Lojas em Geral \& Supermercado } \\
\text { \& Vestuario }\end{array}$ & 2,93 & 0,00 & 35,81 & 1,02 \\
\hline 496 & Diversao_1 \& Supermercado_1 & Postos de Gasolina_1 \& Farmacia & 2,93 & 0,11 & 20,52 & 1,40 \\
\hline 497 & Postos de Gasolina_1 \& Farmacia & Diversao_1\& Supermercado_1 & 2,93 & 0,11 & 20,05 & 1,40 \\
\hline 498 & Diversao & $\begin{array}{l}\text { Farmacia \& Lojas em Geral \& Supermercado } \\
\& \text { Vestuario }\end{array}$ & 2,93 & 0,00 & 36,59 & 1,04 \\
\hline 499 & Diversao & $\begin{array}{l}\text { Postos de Gasolina_1 \& Supermercado \& } \\
\text { Vestuario }\end{array}$ & 2,93 & 0,00 & 36,58 & 1,30 \\
\hline 500 & $\begin{array}{l}\text { Postos de Gasolina_1 \& Supermercado \& } \\
\text { Vestuario }\end{array}$ & Diversao & 2,93 & 0,00 & 10,37 & 1,30 \\
\hline 501 & Diversao & $\begin{array}{l}\text { Postos de Gasolina \& Supermercado \& } \\
\text { Vestuario }\end{array}$ & 2,92 & 0,00 & 36,52 & 1,34 \\
\hline 502 & $\begin{array}{l}\text { Postos de Gasolina \& Supermercado \& } \\
\text { Vestuario }\end{array}$ & Diversao & 2,92 & 0,00 & 10,70 & 1,34 \\
\hline 505 & Alimentos \& Lojas em Geral & Farmacia \& Vestuario & 2,91 & 0,00 & 10,02 & 1,02 \\
\hline 507 & Supermercado_1 \& Diversao & Postos de Gasolina_1 \& Farmacia & 2,91 & 0,10 & 20,79 & 1,42 \\
\hline 508 & Postos de Gasolina_1 \& Farmacia & Supermercado_1 \& Diversao & 2,91 & 0,10 & 19,93 & 1,42 \\
\hline 511 & Diversao_1\& Supermercado_1 & Farmacia \& Postos de Gasolina & 2,91 & 0,10 & 20,37 & 1,44 \\
\hline 512 & Farmacia \& Postos de Gasolina & Diversao_1\& Supermercado_1 & 2,91 & 0,10 & 20,54 & 1,44 \\
\hline 514 & Postos de Gasolina_1 & Veiculos_1 \& Supermercado & 2,90 & 0,23 & 57,04 & 1,12 \\
\hline 520 & Postos de Gasolina & Veiculos_1 \& Supermercado & 2,90 & 0,23 & 58,68 & 1,15 \\
\hline 522 & Farmacia_1 \& Postos de Gasolina_1 & Diversao_1 \& Supermercado & 2,89 & 0,12 & 15,88 & 1,21 \\
\hline 523 & Diversao_1 \& Supermercado & Farmacia_1 \& Postos de Gasolina_1 & 2,89 & 0,12 & 22,05 & 1,21 \\
\hline 524 & Diversao_1 & $\begin{array}{l}\text { Farmacia_1 } 8 \text { Postos de Gasolina_1 \& } \\
\text { Supermercado_1 }\end{array}$ & 2,89 & 0,23 & 35,31 & 1,15 \\
\hline 525 & Farmacia \& Postos de Gasolina & Supermercado_1 \& Diversao & 2,89 & 0,09 & 20,42 & 1,46 \\
\hline 526 & Supermercado_1 \& Diversao & Farmacia \& Postos de Gasolina & 2,89 & 0,09 & 20,64 & 1,46 \\
\hline 527 & Farmacia_1 \& Postos de Gasolina & Diversao_1 \& Supermercado & 2,89 & 0,11 & 16,26 & 1,24 \\
\hline 528 & Diversao_1 \& Supermercado & Farmacia_1 \& Postos de Gasolina & 2,89 & 0,11 & 22,03 & 1,24 \\
\hline
\end{tabular}




\begin{tabular}{|c|c|c|c|c|c|c|}
\hline 534 & Diversao_1 \& Farmacia_1 & Postos de Gasolina_1 \& Supermercado_1 & 2,89 & 0,23 & 11,59 & 1,15 \\
\hline 535 & Postos de Gasolina_1 \& Supermercado_1 & Diversao_1 \& Farmacia_1 & 2,89 & 0,23 & 28,52 & 1,15 \\
\hline 536 & Diversao_1 & $\begin{array}{l}\text { Farmacia_1 \& Supermercado_1 \& Postos de } \\
\text { Gasolina }\end{array}$ & 2,88 & 0,23 & 35,22 & 1,17 \\
\hline 537 & Postos de Gasolina_1 & Farmacia_1\& Veiculos & 2,88 & 0,09 & 56,69 & 1,09 \\
\hline 540 & Diversao \& Farmacia \& Supermercado & Lojas em Geral \& Vestuario & 2,88 & 0,00 & 10,39 & 1,04 \\
\hline 544 & Postos de Gasolina & Farmacia_1 \& Veiculos & 2,88 & 0,09 & 58,27 & 1,12 \\
\hline 546 & Diversao_1 \& Farmacia \& Supermercado & Lojas em Geral \& Vestuario & 2,87 & 0,00 & 10,17 & 1,02 \\
\hline 547 & Diversao \& Supermercado & Farmacia_1 \& Postos de Gasolina_1 & 2,87 & 0,11 & 22,34 & 1,23 \\
\hline 548 & Farmacia_1 \& Postos de Gasolina_1 & Diversao \& Supermercado & 2,87 & 0,11 & 15,77 & 1,23 \\
\hline 550 & Farmacia_1 \& Diversao & Postos de Gasolina_1 \& Supermercado_1 & 2,87 & 0,22 & 11,74 & 1,16 \\
\hline 551 & Postos de Gasolina_1 \& Supermercado_1 & Farmacia_1 \& Diversao & 2,87 & 0,22 & 28,34 & 1,16 \\
\hline 552 & Diversao \& Supermercado & Farmacia_1 \& Postos de Gasolina & 2,87 & 0,10 & 22,31 & 1,26 \\
\hline 553 & Farmacia_1\& Postos de Gasolina & Diversao \& Supermercado & 2,87 & 0,10 & 16,14 & 1,26 \\
\hline 556 & Diversao_1 \& Supermercado & Postos de Gasolina_1 \& Farmacia & 2,87 & 0,01 & 21,85 & 1,50 \\
\hline 557 & Postos de Gasolina_1 \& Farmacia & Diversao_1 \& Supermercado & 2,87 & 0,01 & 19,62 & 1,50 \\
\hline 558 & Supermercado_1 \& Postos de Gasolina & Diversao_1 \& Farmacia_1 & 2,86 & 0,23 & 29,14 & 1,17 \\
\hline 559 & Diversao_1 \& Farmacia_1 & Supermercado_1 \& Postos de Gasolina & 2,86 & 0,23 & 11,51 & 1,17 \\
\hline 560 & $\begin{array}{l}\text { Postos de Gasolina_1 \& Supermercado_1 \& } \\
\text { Farmacia }\end{array}$ & Diversao_1 & 2,86 & 0,11 & 11,50 & 1,40 \\
\hline 561 & Diversao_1 & $\begin{array}{l}\text { Postos de Gasolina_1 \& Supermercado_1 \& } \\
\text { Farmacia }\end{array}$ & 2,86 & 0,11 & 34,94 & 1,40 \\
\hline 562 & Diversao & $\begin{array}{l}\text { Farmacia_1 \& Postos de Gasolina_1 \& } \\
\text { Supermercado_1 }\end{array}$ & 2,86 & 0,22 & 35,76 & 1,16 \\
\hline 566 & Diversao & $\begin{array}{l}\text { Farmacia_1 \& Supermercado_1 \& Postos de } \\
\text { Gasolina }\end{array}$ & 2,85 & 0,21 & 35,66 & 1,19 \\
\hline 567 & Diversao_1 \& Supermercado & Farmacia \& Postos de Gasolina & 2,85 & 0,01 & 21,75 & 1,54 \\
\hline 568 & Farmacia \& Postos de Gasolina & Diversao_1 \& Supermercado & 2,85 & 0,01 & 20,15 & 1,54 \\
\hline 570 & Postos de Gasolina_1 \& Farmacia & Diversao \& Supermercado & 2,85 & 0,00 & 19,49 & 1,52 \\
\hline 571 & Diversao \& Supermercado & Postos de Gasolina_1 \& Farmacia & 2,85 & 0,00 & 22,16 & 1,52 \\
\hline 572 & Supermercado_1 \& Postos de Gasolina & Farmacia_1 \& Diversao & 2,85 & 0,21 & 28,96 & 1,19 \\
\hline 573 & Farmacia_1\& Diversao & Supermercado_1 \& Postos de Gasolina & 2,85 & 0,21 & 11,65 & 1,19 \\
\hline 574 & Diversao_1 & $\begin{array}{l}\text { Supermercado_1 \& Farmacia \& Postos de } \\
\text { Gasolina }\end{array}$ & 2,85 & 0,10 & 34,75 & 1,44 \\
\hline 575 & $\begin{array}{l}\text { Supermercado_1 \& Farmacia \& Postos de } \\
\text { Gasolina }\end{array}$ & Diversao_1 & 2,85 & 0,10 & 11,78 & 1,44 \\
\hline 576 & Diversao_1 & $\begin{array}{l}\text { Farmacia_1 \& Postos de Gasolina_1 \& } \\
\text { Supermercado }\end{array}$ & 2,84 & 0,12 & 34,73 & 1,21 \\
\hline 578 & Diversao_1 \& Vestuario & Lojas em Geral \& Supermercado & 2,84 & 0,00 & 14,37 & 1,73 \\
\hline 582 & Diversao_1 & $\begin{array}{l}\text { Farmacia_1 \& Postos de Gasolina \& } \\
\text { Supermercado }\end{array}$ & 2,84 & 0,11 & 34,66 & 1,24 \\
\hline 583 & $\begin{array}{l}\text { Farmacia_1 \& Postos de Gasolina \& } \\
\text { Supermercado }\end{array}$ & Diversao_1 & 2,84 & 0,11 & 10,16 & 1,24 \\
\hline 585 & Diversao_1 \& Vestuario & Farmacia \& Postos de Gasolina & 2,84 & 0,01 & 14,34 & 1,01 \\
\hline 586 & Farmacia \& Postos de Gasolina & Diversao_1 \& Vestuario & 2,84 & 0,01 & 20,03 & 1,01 \\
\hline 589 & Farmacia \& Postos de Gasolina & Diversao \& Supermercado & 2,83 & 0,00 & 20,02 & 1,56 \\
\hline 590 & Diversao \& Supermercado & Farmacia \& Postos de Gasolina & 2,83 & 0,00 & 22,05 & 1,56 \\
\hline 593 & $\begin{array}{l}\text { Postos de Gasolina_1 \& Supermercado_1 \& } \\
\text { Farmacia }\end{array}$ & Diversao & 2,83 & 0,10 & 11,39 & 1,42 \\
\hline
\end{tabular}

Tabela 47: Regras de associação da ACC utilizadas na segmentação 


\section{Regras das AAs}

\begin{tabular}{|c|c|c|c|c|c|c|c|}
\hline Visão & Análise & $\begin{array}{l}\text { ID da } \\
\text { Regra }\end{array}$ & Consequênte & Antecedente & Lift & $\begin{array}{l}\text { Confidence } \\
(\%)\end{array}$ & Support(\%) \\
\hline Mensal & $\begin{array}{l}\text { Família de } \\
\text { Produto }\end{array}$ & 1 & Postos de Gasolina & Veículos \& Diversão & 3,18 & 64,4 & 0,95 \\
\hline Mensal & $\begin{array}{l}\text { Família de } \\
\text { Produto }\end{array}$ & 2 & Postos de Gasolina \& Construção & Veículos & 2,96 & 8,49 & 0,7 \\
\hline Mensal & $\begin{array}{l}\text { Família de } \\
\text { Produto }\end{array}$ & 6 & Serviços \& Postos de Gasolina & Diversão & 2,89 & 6,66 & 0,83 \\
\hline Mensal & $\begin{array}{l}\text { Família de } \\
\text { Produto }\end{array}$ & 8 & Postos de Gasolina & Veículos \& Alimentos & 2,89 & 58,43 & 0,64 \\
\hline Mensal & $\begin{array}{l}\text { Família de } \\
\text { Produto }\end{array}$ & 12 & Postos de Gasolina \& Alimentos & Diversão & 2,77 & 8,2 & 1,03 \\
\hline Mensal & $\begin{array}{l}\text { Família de } \\
\text { Produto }\end{array}$ & 15 & Telecomunicação / Eletrônicos \& Postos de Gasolina & Veículos & 2,75 & 7,37 & 0,6 \\
\hline Mensal & $\begin{array}{l}\text { Família de } \\
\text { Produto }\end{array}$ & 18 & Postos de Gasolina & Veículos \& Farmácia & 2,74 & 55,47 & 1,4 \\
\hline Mensal & $\begin{array}{l}\text { Família de } \\
\text { Produto }\end{array}$ & 25 & Postos de Gasolina \& Alimentos & Veículos & 2,63 & 7,79 & 0,64 \\
\hline Mensal & $\begin{array}{l}\text { Família de } \\
\text { Produto }\end{array}$ & 27 & Postos de Gasolina \& Lojas em Geral & Diversão & 2,62 & 13,3 & 1,66 \\
\hline Mensal & $\begin{array}{l}\text { Família de } \\
\text { Produto }\end{array}$ & 30 & Postos de Gasolina & Veículos \& Construção & 2,6 & 52,69 & 0,7 \\
\hline Mensal & $\begin{array}{l}\text { Família de } \\
\text { Produto }\end{array}$ & 31 & Postos de Gasolina \& Diversão & Veículos & 2,59 & 11,58 & 0,95 \\
\hline Mensal & $\begin{array}{l}\text { Família de } \\
\text { Produto }\end{array}$ & 35 & Postos de Gasolina \& Lojas em Geral & Veículos & 2,58 & 13,13 & 1,08 \\
\hline Mensal & $\begin{array}{l}\text { Família de } \\
\text { Produto }\end{array}$ & 45 & Telecomunicação / Eletrônicos \& Postos de Gasolina & Diversão & 2,54 & 6,82 & 0,85 \\
\hline Mensal & $\begin{array}{l}\text { Família de } \\
\text { Produto }\end{array}$ & 48 & Postos de Gasolina & Veículos \& Supermercado & 2,53 & 51,3 & 2,05 \\
\hline Mensal & $\begin{array}{l}\text { Família de } \\
\text { Produto }\end{array}$ & 54 & Vestuário \& Postos de Gasolina & Diversão & 2,51 & 14,69 & 1,84 \\
\hline Mensal & $\begin{array}{l}\text { Família de } \\
\text { Produto }\end{array}$ & 57 & Postos de Gasolina & Veículos \& Lojas em Geral & 2,51 & 50,78 & 1,08 \\
\hline Mensal & $\begin{array}{l}\text { Família de } \\
\text { Produto }\end{array}$ & 60 & Postos de Gasolina & Veículos \& Telecomunicação / Eletrônicos & 2,49 & 50,46 & 0,6 \\
\hline Mensal & $\begin{array}{l}\text { Família de } \\
\text { Produto }\end{array}$ & 74 & Postos de Gasolina \& Farmácia & Diversão & 2,43 & 17,16 & 2,14 \\
\hline
\end{tabular}




\begin{tabular}{|c|c|c|c|c|c|c|c|}
\hline Mensal & $\begin{array}{l}\text { Família de } \\
\text { Produto }\end{array}$ & 76 & Postos de Gasolina \& Farmácia & Veículos & 2,42 & 17,09 & 1,4 \\
\hline Mensal & $\begin{array}{l}\text { Família de } \\
\text { Produto }\end{array}$ & 118 & Vestuário \& Postos de Gasolina & Veículos & 2,3 & 13,44 & 1,1 \\
\hline Mensal & $\begin{array}{l}\text { Família de } \\
\text { Produto }\end{array}$ & 123 & Postos de Gasolina & Veículos \& Vestuário & 2,29 & 46,47 & 1,1 \\
\hline Mensal & $\begin{array}{l}\text { Família de } \\
\text { Produto }\end{array}$ & 151 & Supermercado \& Postos de Gasolina & Veículos & 2,22 & 25,03 & 2,05 \\
\hline Mensal & $\begin{array}{l}\text { Família de } \\
\text { Produto }\end{array}$ & 176 & Postos de Gasolina & Diversão \& Construção & 2,15 & 43,55 & 0,77 \\
\hline Mensal & $\begin{array}{l}\text { Família de } \\
\text { Produto }\end{array}$ & 181 & Postos de Gasolina & Veículos & 2,14 & 43,38 & 3,55 \\
\hline Mensal & $\begin{array}{l}\text { Família de } \\
\text { Produto }\end{array}$ & 185 & Postos de Gasolina \& Construção & Diversão & 2,14 & 6,13 & 0,77 \\
\hline Mensal & $\begin{array}{l}\text { Família de } \\
\text { Produto }\end{array}$ & 187 & Veículos \& Postos de Gasolina & Diversão & 2,14 & 7,59 & 0,95 \\
\hline Mensal & $\begin{array}{l}\text { Família de } \\
\text { Produto }\end{array}$ & 192 & Supermercado \& Postos de Gasolina & Diversão & 2,13 & 23,94 & 2,99 \\
\hline Mensal & $\begin{array}{l}\text { Família de } \\
\text { Produto }\end{array}$ & 200 & Postos de Gasolina \& Diversão & Alimentos & 2,12 & 9,46 & 1,03 \\
\hline Mensal & $\begin{array}{l}\text { Família de } \\
\text { Produto }\end{array}$ & 206 & Vestuário \& Postos de Gasolina & Joalherias & 2,12 & 12,38 & 0,61 \\
\hline Mensal & $\begin{array}{l}\text { Família de } \\
\text { Produto }\end{array}$ & 241 & Postos de Gasolina \& Diversão & Serviços & 2,07 & 9,22 & 0,83 \\
\hline Mensal & $\begin{array}{l}\text { Família de } \\
\text { Produto }\end{array}$ & 269 & Postos de Gasolina & Diversão \& Alimentos & 2,03 & 41,13 & 1,03 \\
\hline Mensal & $\begin{array}{l}\text { Família de } \\
\text { Produto }\end{array}$ & 271 & Postos de Gasolina & Farmácia \& Diversão & 2,03 & 41,03 & 2,14 \\
\hline Mensal & $\begin{array}{l}\text { Família de } \\
\text { Produto }\end{array}$ & 296 & Postos de Gasolina \& Lojas em Geral & Geral & 2,01 & 10,19 & 0,59 \\
\hline Mensal & Produto & 87 & $\begin{array}{l}\text { Postos de Gasolina_1_F02:18|--30 \& Diversao_1_F03:19|-- } \\
31\end{array}$ & Diversao_1_F02:12|--19 & 7,78 & 5,43 & 0,2 \\
\hline Mensal & Produto & 94 & Postos de Gasolina_1_F02:18|--30 & $\begin{array}{l}\text { Postos de Gasolina_1_F03:30|--50 \& Postos de } \\
\text { Gasolina_1_F01:0|--18 }\end{array}$ & 7,75 & 51,81 & 0,58 \\
\hline Mensal & Produto & 110 & Postos de Gasolina_1_F02:18|--30 & $\begin{array}{l}\text { Postos de Gasolina_1_F04:50|--62 \& Postos de } \\
\text { Gasolina_1_F01:0|--18 }\end{array}$ & 7,23 & 48,33 & 0,23 \\
\hline Mensal & Produto & 141 & $\begin{array}{l}\text { Postos de Gasolina_1_F04:50|--62 \& Postos de } \\
\text { Gasolina_1_F02:18|--30 }\end{array}$ & Postos de Gasolina_1_F03:30|--50 & 6,64 & 5,55 & 0,4 \\
\hline Mensal & Produto & 152 & Postos de Gasolina_1_F02:18|--30 & Postos de Gasolina_1_F01:0|--18 \& Diversao_1_F02:12|--19 & 6,29 & 42,03 & 0,21 \\
\hline Mensal & Produto & 158 & Postos de Gasolina_1_F02:18|--30 & Postos de Gasolina_1_F01:0|--18 \& Diversao_1_F03:19|--31 & 6,15 & 41,07 & 0,25 \\
\hline
\end{tabular}




\begin{tabular}{|c|c|c|c|c|c|c|c|}
\hline Mensal & Produto & 163 & $\begin{array}{l}\text { Supermercado_1_F01:0|--13 \& Postos de } \\
\text { Gasolina_1_F02:18|--30 }\end{array}$ & Postos de Gasolina_1_F01:0|--18 & 6,11 & 8,5 & 0,48 \\
\hline Mensal & Produto & 170 & Postos de Gasolina_1_F02:18|--30 & Postos de Gasolina_1_F01:0|--18 \& Farmacia_1_F03:17|--27 & 6,03 & 40,32 & 0,24 \\
\hline Mensal & Produto & 180 & $\begin{array}{l}\text { Supermercado_1_F02:13|--28 \& Postos de } \\
\text { Gasolina } 1 \text { F02:18|--30 }\end{array}$ & Postos de Gasolina_1_F01:0|--18 & 5,76 & 9,54 & 0,54 \\
\hline Mensal & Produto & 182 & Postos de Gasolina_1_F02:18|--30 & Postos de Gasolina_1_F03:30|--50 \& Diversao_1_F02:12|--19 & 5,75 & 38,43 & 0,22 \\
\hline Mensal & Produto & 187 & Postos de Gasolina_1_F02:18|--30 & Postos de Gasolina_1_F01:0|--18 \& Farmacia_1_F01:0|--11 & 5,69 & 38,03 & 0,27 \\
\hline Mensal & Produto & 188 & Postos de Gasolina_1_F02:18|--30 & Postos de Gasolina_1_F03:30|--50 \& Diversao_1_F03:19|--31 & 5,62 & 37,57 & 0,27 \\
\hline Mensal & Produto & 189 & Postos de Gasolina_1_F02:18|--30 & $\begin{array}{l}\text { Supermercado_1_F04:61|--122 \& Postos de } \\
\text { Gasolina 1 F01:0|--18 }\end{array}$ & 5,6 & 37,44 & 0,26 \\
\hline Mensal & Produto & 190 & Postos de Gasolina_1_F02:18|--30 & Postos de Gasolina_1_F01:0|--18 \& Farmacia_1_F02:11|--17 & 5,6 & 37,4 & 0,24 \\
\hline Mensal & Produto & 195 & Postos de Gasolina_1_F02:18|--30 & $\begin{array}{l}\text { Supermercado_1_F02:13|--28 \& Postos de Gasolina_1_F01:0|- } \\
\text {-18 }\end{array}$ & 5,52 & 36,91 & 0,54 \\
\hline Mensal & Produto & 197 & Postos de Gasolina_1_F02:18|--30 & $\begin{array}{l}\text { Supermercado_1_F03:28|--61 \& Postos de Gasolina_1_F01:0|- } \\
-18\end{array}$ & 5,47 & 36,55 & 0,48 \\
\hline Mensal & Produto & 200 & $\begin{array}{l}\text { Postos de Gasolina_1_F03:30|--50 \& Postos de } \\
\text { Gasolina_1_F02:18|--30 }\end{array}$ & Postos de Gasolina_1_F01:0|--18 & 5,4 & 10,37 & 0,58 \\
\hline Mensal & Produto & 210 & $\begin{array}{l}\text { Supermercado_1_F03:28|--61 \& Postos de } \\
\text { Gasolina_1_F02:18|--30 }\end{array}$ & Postos de Gasolina_1_F01:0|--18 & 5,28 & 8,46 & 0,48 \\
\hline Mensal & Produto & 217 & Postos de Gasolina_1_F02:18|--30 & Postos de Gasolina_1_F01:0|--18 & 5,1 & 34,08 & 1,92 \\
\hline Mensal & Produto & 219 & Postos de Gasolina_1_F02:18|--30 & $\begin{array}{l}\text { Supermercado_1_F01:0|--13 \& Postos de Gasolina_1_F01:0|-- } \\
18\end{array}$ & 5,08 & 33,92 & 0,48 \\
\hline Mensal & Produto & 227 & Postos de Gasolina_1_F02:18|--30 & Postos de Gasolina_1_F03:30|--50 \& Farmacia_1_F02:11|--17 & 4,92 & 32,86 & 0,23 \\
\hline Mensal & Produto & 241 & Postos de Gasolina_1_F02:18|--30 & $\begin{array}{l}\text { Supermercado_1_F02:13|--28 \& Postos de } \\
\text { Gasolina_1_F03:30|--50 }\end{array}$ & 4,81 & 32,11 & 0,55 \\
\hline Mensal & Produto & 242 & Postos de Gasolina_1_F02:18|--30 & Postos de Gasolina_1_F03:30|--50 \& Farmacia_1_F03:17|--27 & 4,79 & 32,01 & 0,25 \\
\hline Mensal & Produto & 244 & Postos de Gasolina_1_F02:18|--30 & $\begin{array}{l}\text { Postos de Gasolina_1_F04:50|--62 \& Postos de } \\
\text { Gasolina_1_F03:30|--50 }\end{array}$ & 4,77 & 31,88 & 0,4 \\
\hline Mensal & Produto & 245 & Postos de Gasolina_1_F02:18|--30 & Postos de Gasolina_1_F03:30|--50 \& Farmacia_1_F01:0|--11 & 4,77 & 31,87 & 0,21 \\
\hline Mensal & Produto & 252 & $\begin{array}{l}\text { Postos de Gasolina_1_F03:30|--50 \& Postos de } \\
\text { Gasolina_1_F02:18|--30 }\end{array}$ & Postos de Gasolina_1_F04:50|--62 & 4,69 & 9,02 & 0,4 \\
\hline Mensal & Produto & 259 & Postos de Gasolina_1_F02:18|--30 & $\begin{array}{l}\text { Supermercado_1_F01:0|--13 \& Postos de Gasolina_1_F03:30|- } \\
-50\end{array}$ & 4,68 & 31,24 & 0,43 \\
\hline Mensal & Produto & 265 & $\begin{array}{l}\text { Supermercado_1_F02:13|--28 \& Postos de } \\
\text { Gasolina_1_F02:18|--30 }\end{array}$ & Postos de Gasolina_1_F03:30|--50 & 4,6 & 7,62 & 0,55 \\
\hline Mensal & Produto & 269 & $\begin{array}{l}\text { Supermercado_1_F03:28|--61 \& Postos de } \\
\text { Gasolina_1_F02:18|--30 }\end{array}$ & Postos de Gasolina_1_F03:30|--50 & 4,58 & 7,33 & 0,52 \\
\hline Mensal & Cidade & 35 & Salvador & Dias d Ávila & 6,53 & 78,51 & 0,17 \\
\hline
\end{tabular}




\begin{tabular}{|c|c|c|c|c|c|c|c|}
\hline Mensal & Cidade & 36 & Salvador & Lauro de Freitas & 6,49 & 77,97 & 0,59 \\
\hline Mensal & Cidade & 37 & Salvador & Simões Filho & 6,45 & 77,51 & 0,26 \\
\hline Mensal & Cidade & 38 & Salvador & Candeias & 6,01 & 72,21 & 0,19 \\
\hline Mensal & Cidade & 42 & Salvador & Camaçari & 5,28 & 63,52 & 0,41 \\
\hline Anual & $\begin{array}{l}\text { Família de } \\
\text { Produto }\end{array}$ & 7 & Postos de Gasolina \& Diversão & Viagens & 2,45 & 42,24 & 3,53 \\
\hline Anual & $\begin{array}{l}\text { Família de } \\
\text { Produto }\end{array}$ & 13 & Viagens \& Postos de Gasolina & Diversão & 2,41 & 11,74 & 3,53 \\
\hline Anual & $\begin{array}{l}\text { Família de } \\
\text { Produto }\end{array}$ & 17 & Viagens \& Postos de Gasolina & Veículos & 2,4 & 11,7 & 3,02 \\
\hline Anual & $\begin{array}{l}\text { Família de } \\
\text { Produto }\end{array}$ & 37 & Serviços \& Postos de Gasolina & Viagens & 2,32 & 31,5 & 2,63 \\
\hline Anual & $\begin{array}{l}\text { Família de } \\
\text { Produto }\end{array}$ & 72 & Postos de Gasolina & Viagens \& Veículos & 2,27 & 82,47 & 3,02 \\
\hline Anual & $\begin{array}{l}\text { Família de } \\
\text { Produto }\end{array}$ & 119 & Postos de Gasolina \& Construção & Veículos & 2,2 & 37,8 & 9,76 \\
\hline Anual & $\begin{array}{l}\text { Família de } \\
\text { Produto }\end{array}$ & 128 & Postos de Gasolina & Veículos \& Diversão & 2,2 & 80,06 & 9,09 \\
\hline Anual & $\begin{array}{l}\text { Família de } \\
\text { Produto }\end{array}$ & 155 & Postos de Gasolina \& Livraria & Diversão & 2,18 & 21,25 & 6,38 \\
\hline Anual & $\begin{array}{l}\text { Família de } \\
\text { Produto }\end{array}$ & 232 & Postos de Gasolina & Veículos \& Alimentos & 2,14 & 77,96 & 6,87 \\
\hline Anual & $\begin{array}{l}\text { Família de } \\
\text { Produto }\end{array}$ & 243 & Postos de Gasolina \& Perfumaria & Diversão & 2,14 & 23,39 & 7,03 \\
\hline Anual & $\begin{array}{l}\text { Família de } \\
\text { Produto }\end{array}$ & 249 & Veículos \& Postos de Gasolina & Viagens & 2,13 & 36,2 & 3,02 \\
\hline Anual & $\begin{array}{l}\text { Família de } \\
\text { Produto }\end{array}$ & 251 & Postos de Gasolina \& Móveis & Veículos & 2,13 & 16,98 & 4,39 \\
\hline Anual & $\begin{array}{l}\text { Família de } \\
\text { Produto }\end{array}$ & 259 & Telecomunicação / Eletrônicos \& Postos de Gasolina & Veículos & 2,13 & 35,83 & 9,25 \\
\hline Anual & $\begin{array}{l}\text { Família de } \\
\text { Produto }\end{array}$ & 265 & Telecomunicação / Eletrônicos \& Postos de Gasolina & Viagens & 2,12 & 35,79 & 2,99 \\
\hline Anual & $\begin{array}{l}\text { Família de } \\
\text { Produto }\end{array}$ & 289 & Postos de Gasolina \& Alimentos & Diversão & 2,12 & 27,89 & 8,38 \\
\hline Anual & $\begin{array}{l}\text { Família de } \\
\text { Produto }\end{array}$ & 319 & Postos de Gasolina \& Joalherias & Diversão & 2,1 & 21,09 & 6,34 \\
\hline Anual & $\begin{array}{l}\text { Família de } \\
\text { Produto }\end{array}$ & 323 & Postos de Gasolina \& Perfumaria & Livraria & 2,1 & 22,97 & 4,44 \\
\hline Anual & $\begin{array}{l}\text { Família de } \\
\text { Produto }\end{array}$ & 331 & Postos de Gasolina \& Geral & Veículos & 2,09 & 22,62 & 5,84 \\
\hline
\end{tabular}

133 


\begin{tabular}{|c|c|c|c|c|c|c|c|}
\hline Anual & $\begin{array}{l}\text { Família de } \\
\text { Produto }\end{array}$ & 353 & Postos de Gasolina \& Perfumaria & Joalherias & 2,08 & 22,81 & 4,92 \\
\hline Anual & $\begin{array}{l}\text { Familia de } \\
\text { Produto }\end{array}$ & 355 & Serviços \& Postos de Gasolina & Diversão & 2,08 & 28,29 & 8,5 \\
\hline Anual & $\begin{array}{l}\text { Família de } \\
\text { Produto }\end{array}$ & 367 & Postos de Gasolina \& Joalherias & Perfumaria & 2,08 & 20,87 & 4,92 \\
\hline Anual & $\begin{array}{l}\text { Família de } \\
\text { Produto }\end{array}$ & 391 & Serviços \& Postos de Gasolina & Veículos & 2,07 & 28,08 & 7,25 \\
\hline Anual & $\begin{array}{l}\text { Familia de } \\
\text { Produto }\end{array}$ & 415 & Postos de Gasolina \& Joalherias & Livraria & 2,06 & 20,69 & 4 \\
\hline Anual & $\begin{array}{l}\text { Família de } \\
\text { Produto }\end{array}$ & 426 & Postos de Gasolina & Veículos \& Geral & 2,06 & 74,9 & 5,84 \\
\hline Anual & $\begin{array}{l}\text { Famillia de } \\
\text { Produto }\end{array}$ & 457 & Postos de Gasolina \& Diversão & Veículos & 2,04 & 35,19 & 9,09 \\
\hline Anual & $\begin{array}{l}\text { Familia de } \\
\text { Produto }\end{array}$ & 479 & Postos de Gasolina \& Joalherias & Veículos & 2,03 & 20,38 & 5,26 \\
\hline Anual & $\begin{array}{l}\text { Família de } \\
\text { Produto }\end{array}$ & 511 & Postos de Gasolina \& Alimentos & Veículos & 2,02 & 26,6 & 6,87 \\
\hline Anual & $\begin{array}{l}\text { Famillia de } \\
\text { Produto }\end{array}$ & 554 & Postos de Gasolina & Veículos \& Livraria & 2 & 72,75 & 5,01 \\
\hline Anual & Produto & 153 & $\begin{array}{l}\text { Postos de Gasolina_1_F02:18|--30 \& Diversao_1_F05:45|-- } \\
71\end{array}$ & Diversao_1_F04:31|--45 & 5,95 & 15,8 & 1,61 \\
\hline Anual & Produto & 167 & $\begin{array}{l}\text { Postos de Gasolina_1_F02:18|--30 \& Diversao_1_F04:31|-- } \\
45\end{array}$ & Diversao_1_F05:45|--71 & 5,89 & 23,14 & 1,61 \\
\hline Anual & Produto & 181 & $\begin{array}{l}\text { Postos de Gasolina_1_F05:62|--94 \& Postos de } \\
\text { Gasolina_1_F02:18|--30 }\end{array}$ & Postos de Gasolina_1_F04:50|--62 & 5,79 & 20,32 & 2,59 \\
\hline Anual & Produto & 481 & $\begin{array}{l}\text { Postos de Gasolina_1_F02:18|--30 \& Alimentos_1_F02:12|-- } \\
21\end{array}$ & Alimentos_1_F01:0|--12 & 5,23 & 18,59 & 1,78 \\
\hline Anual & Produto & 485 & $\begin{array}{l}\text { Postos de Gasolina_1_F04:50|--62 \& Postos de } \\
\text { Gasolina_1_F02:18|--30 }\end{array}$ & Postos de Gasolina_1_F05:62|--94 & 5,23 & 33,66 & 2,59 \\
\hline Anual & Produto & 489 & Veiculos_1_F01:0|--16 \& Postos de Gasolina_1_F02:18|--30 & Postos de Gasolina_1_F01:0|--18 & 5,22 & 13,66 & 1,98 \\
\hline Anual & Produto & 547 & $\begin{array}{l}\text { Postos de Gasolina_1_F02:18|--30 \& Alimentos_1_F01:0|-- } \\
12\end{array}$ & Alimentos_1_F02:12|--21 & 5,17 & 15,53 & 1,78 \\
\hline Anual & Produto & 755 & Postos de Gasolina_1_F02:18|--30 \& Diversao_1_F01:0|--12 & Diversao_1_F02:12|--19 & 4,97 & 20,27 & 2,61 \\
\hline Anual & Produto & 871 & Veiculos_1_F02:16|--27 \& Postos de Gasolina_1_F02:18|--30 & Postos de Gasolina_1_F01:0|--18 & 4,88 & 13,56 & 1,97 \\
\hline Anual & Produto & 983 & $\begin{array}{l}\text { Postos de Gasolina_1_F02:18|--30 \& Alimentos_1_F04:39|-- } \\
82\end{array}$ & Alimentos_1_F03:21|--39 & 4,82 & 12,42 & 1,51 \\
\hline Anual & Produto & 1063 & $\begin{array}{l}\text { Postos de Gasolina_1_F02:18|--30 \& Diversao_1_F03:19|-- } \\
31\end{array}$ & Diversao_1_F05:45|--71 & 4,77 & 27,21 & 1,9 \\
\hline Anual & Produto & 1069 & $\begin{array}{l}\text { Postos de Gasolina_1_F02:18|--30 \& Diversao_1_F02:12|-- } \\
19\end{array}$ & Diversao_1_F04:31|--45 & 4,76 & 22,52 & 2,3 \\
\hline
\end{tabular}




\begin{tabular}{|c|c|c|c|c|c|c|c|}
\hline Anual & Produto & 1085 & $\begin{array}{l}\text { Postos de Gasolina_1_F02:18|--30 \& Diversao_1_F03:19|-- } \\
31\end{array}$ & Diversao_1_F04:31|--45 & 4,75 & 27,13 & 2,77 \\
\hline Anual & Produto & 1101 & $\begin{array}{l}\text { Postos de Gasolina_1_F02:18|--30 \& Diversao_1_F02:12|-- } \\
19\end{array}$ & Diversao_1_F01:0|--12 & 4,74 & 22,43 & 2,61 \\
\hline Anual & Produto & 1147 & $\begin{array}{l}\text { Postos de Gasolina_1_F02:18|--30 \& Postos de } \\
\text { Gasolina_1_F01:0|--18 }\end{array}$ & Veiculos_1_F01:0|--16 & 4,71 & 43,91 & 1,98 \\
\hline Anual & Produto & 1163 & $\begin{array}{l}\text { Postos de Gasolina_1_F02:18|--30 \& Alimentos_1_F03:21|-- } \\
39\end{array}$ & Alimentos_1_F02:12|--21 & 4,7 & 16,76 & 1,92 \\
\hline Anual & Produto & 1189 & $\begin{array}{l}\text { Postos de Gasolina_1_F02:18|--30 \& Construcao_1_F01:0|-- } \\
15\end{array}$ & Postos de Gasolina_1_F01:0|--18 & 4,67 & 11,55 & 1,67 \\
\hline Anual & Produto & 1239 & Veiculos_1_F03:27|--50 \& Postos de Gasolina_1_F02:18|--30 & Postos de Gasolina_1_F01:0|--18 & 4,65 & 13,56 & 1,97 \\
\hline Anual & Produto & 1251 & $\begin{array}{l}\text { Postos de Gasolina_1_F02:18|--30 \& Diversao_1_F02:12|-- } \\
19\end{array}$ & Diversao_1_F05:45|--71 & 4,64 & 21,96 & 1,53 \\
\hline Anual & Produto & 1257 & $\begin{array}{l}\text { Postos de Gasolina_1_F02:18|--30 \& Alimentos_1_F03:21|-- } \\
39\end{array}$ & Alimentos_1_F04:39|--82 & 4,64 & 16,54 & 1,51 \\
\hline Anual & Produto & 1311 & $\begin{array}{l}\text { Postos de Gasolina_1_F02:18|--30 \& Diversao_1_F05:45|-- } \\
71\end{array}$ & Diversao_1_F03:19|--31 & 4,61 & 12,24 & 1,9 \\
\hline Anual & Produto & 1343 & $\begin{array}{l}\text { Postos de Gasolina_1_F02:18|--30 \& Alimentos_1_F01:0|-- } \\
12\end{array}$ & Postos de Gasolina_1_F01:0|--18 & 4,58 & 13,77 & 1,99 \\
\hline Anual & Produto & 1405 & $\begin{array}{l}\text { Postos de Gasolina_1_F02:18|--30 \& Diversao_1_F04:31|-- } \\
45\end{array}$ & Diversao_1_F03:19|--31 & 4,55 & 17,87 & 2,77 \\
\hline Anual & Produto & 1415 & $\begin{array}{l}\text { Postos de Gasolina_1_F02:18|--30 \& Diversao_1_F04:31|-- } \\
45\end{array}$ & Diversao_1_F02:12|--19 & 4,54 & 17,86 & 2,3 \\
\hline Anual & Cidade & 33 & Salvador & Simões Filho & 8,23 & 97,42 & 0,71 \\
\hline Anual & Cidade & 38 & Salvador & Lauro de Freitas & 8,09 & 95,85 & 1,55 \\
\hline Anual & Cidade & 42 & Salvador & Camaçari & 7,47 & 88,52 & 1,01 \\
\hline Anual & Cidade & 85 & Salvador & Feira de Santana & 4,39 & 51,98 & 0,78 \\
\hline Anual & Cidade & 102 & Salvador & Vitória da Conquista & 2,06 & 24,35 & 0,56 \\
\hline Mensal & $\begin{array}{l}\text { Família de } \\
\text { Produto }\end{array}$ & 3 & Veículos & Postos de Gasolina \& Construção & 2,96 & 24,25 & 0,7 \\
\hline Mensal & $\begin{array}{l}\text { Família de } \\
\text { Produto }\end{array}$ & 16 & Veículos & Telecomunicação / Eletrônicos \& Postos de Gasolina & 2,75 & 22,53 & 0,6 \\
\hline Mensal & $\begin{array}{l}\text { Família de } \\
\text { Produto }\end{array}$ & 17 & Veículos \& Farmácia & Postos de Gasolina & 2,74 & 6,91 & 1,4 \\
\hline Mensal & $\begin{array}{l}\text { Família de } \\
\text { Produto }\end{array}$ & 26 & Veículos & Postos de Gasolina \& Alimentos & 2,63 & 21,56 & 0,64 \\
\hline Mensal & $\begin{array}{l}\text { Família de } \\
\text { Produto }\end{array}$ & 32 & Veículos & Postos de Gasolina \& Diversão & 2,59 & 21,24 & 0,95 \\
\hline
\end{tabular}




\begin{tabular}{|c|c|c|c|c|c|c|c|}
\hline Mensal & $\begin{array}{l}\text { Família de } \\
\text { Produto }\end{array}$ & 36 & Veículos & Postos de Gasolina \& Lojas em Geral & 2,58 & 21,17 & 1,08 \\
\hline Mensal & $\begin{array}{l}\text { Família de } \\
\text { Produto }\end{array}$ & 47 & Veículos \& Supermercado & Postos de Gasolina & 2,53 & 10,12 & 2,05 \\
\hline Mensal & $\begin{array}{l}\text { Família de } \\
\text { Produto }\end{array}$ & 56 & Veículos \& Lojas em Geral & Postos de Gasolina & 2,51 & 5,31 & 1,08 \\
\hline Mensal & $\begin{array}{l}\text { Família de } \\
\text { Produto }\end{array}$ & 77 & Veículos & Postos de Gasolina \& Farmácia & 2,42 & 19,81 & 1,4 \\
\hline Mensal & $\begin{array}{l}\text { Família de } \\
\text { Produto }\end{array}$ & 119 & Veículos & Vestuário \& Postos de Gasolina & 2,3 & 18,82 & 1,1 \\
\hline Mensal & $\begin{array}{l}\text { Família de } \\
\text { Produto }\end{array}$ & 122 & Veículos \& Vestuário & Postos de Gasolina & 2,29 & 5,44 & 1,1 \\
\hline Mensal & $\begin{array}{l}\text { Família de } \\
\text { Produto }\end{array}$ & 140 & Veículos \& Farmácia & Diversão & 2,25 & 5,67 & 0,71 \\
\hline Mensal & $\begin{array}{l}\text { Família de } \\
\text { Produto }\end{array}$ & 152 & Veículos & Supermercado \& Postos de Gasolina & 2,22 & 18,2 & 2,05 \\
\hline Mensal & $\begin{array}{l}\text { Família de } \\
\text { Produto }\end{array}$ & 177 & Veículos \& Vestuário & Diversão & 2,15 & 5,08 & 0,64 \\
\hline Mensal & $\begin{array}{l}\text { Família de } \\
\text { Produto }\end{array}$ & 182 & Veículos & Postos de Gasolina & 2,14 & 17,55 & 3,55 \\
\hline Mensal & $\begin{array}{l}\text { Família de } \\
\text { Produto }\end{array}$ & 187 & Veículos \& Postos de Gasolina & Diversão & 2,14 & 7,59 & 0,95 \\
\hline Mensal & Cidade & 18 & Recife \& Paulista & Olinda & 14,92 & 13,82 & 0,33 \\
\hline Mensal & Cidade & 20 & Recife \& Olinda & Paulista & 12,69 & 21,24 & 0,33 \\
\hline Mensal & Cidade & 32 & Recife \& Jaboatão dos Guararapes & Cabo de Santo Agostinho & 7,38 & 18,49 & 0,13 \\
\hline Mensal & Cidade & 34 & Recife & Olinda \& Jaboatão dos Guararapes & 6,8 & 85,81 & 0,15 \\
\hline Mensal & Cidade & 39 & Recife & Paulista \& Olinda & 5,68 & 71,7 & 0,33 \\
\hline Mensal & Cidade & 41 & Recife & Olinda & 5,61 & 70,88 & 1,67 \\
\hline Mensal & Cidade & 43 & Recife & Camaragibe & 5,15 & 65 & 0,55 \\
\hline Mensal & Cidade & 45 & Recife & Paulista & 4,78 & 60,3 & 0,93 \\
\hline Mensal & Cidade & 47 & Recife & Jaboatão dos Guararapes & 4,49 & 56,73 & 2,5 \\
\hline Mensal & Cidade & 48 & Recife & Jaboatão dos Guararapes \& Cabo de Santo Agostinho & 4,48 & 56,53 & 0,13 \\
\hline Mensal & Cidade & 49 & Recife & Abreu e Lima & 4,42 & 55,74 & 0,27 \\
\hline Mensal & Cidade & 50 & Recife & Igarassu & 4,25 & 53,71 & 0,14 \\
\hline Mensal & Cidade & 51 & Recife & São Lourenço da Mata & 4,04 & 51,02 & 0,16 \\
\hline Mensal & Cidade & 52 & Recife & Cabo de Santo Agostinho & 3,38 & 42,65 & 0,3 \\
\hline
\end{tabular}




\begin{tabular}{|c|c|c|c|c|c|c|c|}
\hline Mensal & Cidade & 56 & Recife & Vitória de Santo Antão & 2,7 & 34,14 & 0,16 \\
\hline Mensal & Cidade & 57 & Recife \& Jaboatão dos Guararapes & Olinda & 2,5 & 6,27 & 0,15 \\
\hline Anual & $\begin{array}{l}\text { Família de } \\
\text { Produto }\end{array}$ & 1 & Veículos \& Diversão & Viagens & 2,75 & 31,28 & 2,61 \\
\hline Anual & $\begin{array}{l}\text { Família de } \\
\text { Produto }\end{array}$ & 18 & Veículos & Viagens \& Postos de Gasolina & 2,4 & 61,99 & 3,02 \\
\hline Anual & $\begin{array}{l}\text { Família de } \\
\text { Produto }\end{array}$ & 25 & Viagens \& Veículos & Diversão & 2,37 & 8,69 & 2,61 \\
\hline Anual & $\begin{array}{l}\text { Familia de } \\
\text { Produto }\end{array}$ & 71 & Viagens \& Veículos & Postos de Gasolina & 2,27 & 8,3 & 3,02 \\
\hline Anual & $\begin{array}{l}\text { Família de } \\
\text { Produto }\end{array}$ & 89 & Veículos \& Telecomunicação / Eletrônicos & Viagens & 2,24 & 29,53 & 2,47 \\
\hline Anual & $\begin{array}{l}\text { Família de } \\
\text { Produto }\end{array}$ & 111 & Veículos \& Perfumaria & Joalherias & 2,21 & 16,66 & 3,59 \\
\hline Anual & $\begin{array}{l}\text { Família de } \\
\text { Produto }\end{array}$ & 120 & Veículos & Postos de Gasolina \& Construção & 2,2 & 56,9 & 9,76 \\
\hline Anual & $\begin{array}{l}\text { Família de } \\
\text { Produto }\end{array}$ & 127 & Veículos \& Diversão & Postos de Gasolina & 2,2 & 24,97 & 9,09 \\
\hline Anual & $\begin{array}{l}\text { Familia de } \\
\text { Produto }\end{array}$ & 205 & Veículos \& Perfumaria & Livraria & 2,15 & 16,24 & 3,14 \\
\hline Anual & $\begin{array}{l}\text { Família de } \\
\text { Produto }\end{array}$ & 231 & Veículos \& Alimentos & Postos de Gasolina & 2,14 & 18,87 & 6,87 \\
\hline Anual & $\begin{array}{l}\text { Família de } \\
\text { Produto }\end{array}$ & 241 & Veículos \& Livraria & Diversão & 2,14 & 14,7 & 4,42 \\
\hline Anual & $\begin{array}{l}\text { Família de } \\
\text { Produto }\end{array}$ & 249 & Veículos \& Postos de Gasolina & Viagens & 2,13 & 36,2 & 3,02 \\
\hline Anual & $\begin{array}{l}\text { Família de } \\
\text { Produto }\end{array}$ & 252 & Veículos & Postos de Gasolina \& Móveis & 2,13 & 55,07 & 4,39 \\
\hline Anual & $\begin{array}{l}\text { Família de } \\
\text { Produto }\end{array}$ & 260 & Veículos & Telecomunicação / Eletrônicos \& Postos de Gasolina & 2,13 & 54,92 & 9,25 \\
\hline Anual & $\begin{array}{l}\text { Familia de } \\
\text { Produto }\end{array}$ & 332 & Veículos & Postos de Gasolina \& Geral & 2,09 & 54,1 & 5,84 \\
\hline Anual & $\begin{array}{l}\text { Família de } \\
\text { Produto }\end{array}$ & 357 & Veículos \& Alimentos & Diversão & 2,08 & 18,34 & 5,51 \\
\hline Anual & $\begin{array}{l}\text { Família de } \\
\text { Produto }\end{array}$ & 381 & Veículos \& Joalherias & Móveis & 2,07 & 15,43 & 2,72 \\
\hline Anual & $\begin{array}{l}\text { Família de } \\
\text { Produto }\end{array}$ & 392 & Veículos & Serviços \& Postos de Gasolina & 2,07 & 53,35 & 7,25 \\
\hline Anual & $\begin{array}{l}\text { Família de } \\
\text { Produto }\end{array}$ & 405 & Veículos \& Perfumaria & Serviços & 2,06 & 15,55 & 4,24 \\
\hline
\end{tabular}




\begin{tabular}{|c|c|c|c|c|c|c|c|}
\hline Anual & $\begin{array}{l}\text { Família de } \\
\text { Produto }\end{array}$ & 410 & Veículos & Viagens \& Telecomunicação / Eletrônicos & 2,06 & 53,23 & 2,47 \\
\hline Anual & $\begin{array}{l}\text { Família de } \\
\text { Produto }\end{array}$ & 425 & Veículos \& Geral & Postos de Gasolina & 2,06 & 16,05 & 5,84 \\
\hline Anual & $\begin{array}{l}\text { Família de } \\
\text { Produto }\end{array}$ & 437 & Veículos \& Perfumaria & Diversão & 2,05 & 15,46 & 4,64 \\
\hline Anual & $\begin{array}{l}\text { Família de } \\
\text { Produto }\end{array}$ & 441 & Veículos \& Joalherias & Perfumaria & 2,05 & 15,25 & 3,59 \\
\hline Anual & $\begin{array}{l}\text { Família de } \\
\text { Produto }\end{array}$ & 458 & Veículos & Postos de Gasolina \& Diversão & 2,04 & 52,72 & 9,09 \\
\hline Anual & $\begin{array}{l}\text { Família de } \\
\text { Produto }\end{array}$ & 465 & Veículos \& Farmácia & Viagens & 2,04 & 36,24 & 3,03 \\
\hline Anual & $\begin{array}{l}\text { Familia de } \\
\text { Produto }\end{array}$ & 472 & Veículos & Viagens \& Diversão & 2,03 & 52,52 & 2,61 \\
\hline Anual & $\begin{array}{l}\text { Família de } \\
\text { Produto }\end{array}$ & 475 & Veículos \& Joalherias & Serviços & 2,03 & 15,12 & 4,13 \\
\hline Anual & $\begin{array}{l}\text { Família de } \\
\text { Produto }\end{array}$ & 480 & Veículos & Postos de Gasolina \& Joalherias & 2,03 & 52,4 & 5,26 \\
\hline Anual & $\begin{array}{l}\text { Família de } \\
\text { Produto }\end{array}$ & 491 & Veículos \& Joalherias & Livraria & 2,03 & 15,09 & 2,92 \\
\hline Anual & $\begin{array}{l}\text { Família de } \\
\text { Produto }\end{array}$ & 495 & Veículos \& Livraria & Serviços & 2,02 & 13,93 & 3,8 \\
\hline Anual & $\begin{array}{l}\text { Família de } \\
\text { Produto }\end{array}$ & 512 & Veículos & Postos de Gasolina \& Alimentos & 2,02 & 52,14 & 6,87 \\
\hline Anual & $\begin{array}{l}\text { Família de } \\
\text { Produto }\end{array}$ & 523 & Veículos \& Lojas em Geral & Viagens & 2,01 & 36,47 & 3,04 \\
\hline Anual & $\begin{array}{l}\text { Família de } \\
\text { Produto }\end{array}$ & 527 & Veículos \& Diversão & Livraria & 2,01 & 22,83 & 4,42 \\
\hline Anual & $\begin{array}{l}\text { Família de } \\
\text { Produto }\end{array}$ & 553 & Veículos \& Livraria & Postos de Gasolina & 2 & 13,75 & 5,01 \\
\hline Anual & $\begin{array}{l}\text { Família de } \\
\text { Produto }\end{array}$ & 557 & Veículos \& Perfumaria & Geral & 2 & 15,05 & 3,21 \\
\hline Anual & Produto & 9576 & Veiculos_1_F04:50|--96 & Postos de Gasolina_1_F04:50|--62 & 3,03 & 11,1 & 1,41 \\
\hline Anual & Cidade & 5 & Recife \& Abreu e Lima & Paulista & 23,91 & 22,42 & 0,52 \\
\hline Anual & Cidade & 7 & Recife \& Paulista & Abreu e Lima & 23,87 & 51,1 & 0,52 \\
\hline Anual & Cidade & 13 & Recife \& Paulista & Olinda & 15,93 & 34,1 & 1,33 \\
\hline Anual & Cidade & 17 & Recife \& Olinda & Paulista & 15,75 & 57,26 & 1,33 \\
\hline Anual & Cidade & 27 & Recife \& Cabo de Santo Agostinho & Jaboatão dos Guararapes & 10,54 & 10,32 & 0,7 \\
\hline Anual & Cidade & 29 & Recife \& Jaboatão dos Guararapes & Cabo de Santo Agostinho & 10,14 & 59,74 & 0,7 \\
\hline
\end{tabular}




\begin{tabular}{|c|c|c|c|c|c|c|c|}
\hline Anual & Cidade & 39 & São Paulo \& Recife & Olinda & 7,48 & 13,83 & 0,54 \\
\hline Anual & Cidade & 43 & Recife & Paulista \& Jaboatão dos Guararapes & 6,48 & 99,38 & 0,6 \\
\hline Anual & Cidade & 45 & Recife & Olinda \& Jaboatão dos Guararapes & 6,48 & 99,38 & 1,19 \\
\hline Anual & Cidade & 46 & Recife & Jaboatão dos Guararapes \& Camaragibe & 6,42 & 98,5 & 0,49 \\
\hline Anual & Cidade & 47 & Recife & São Paulo \& Olinda & 6,3 & 96,64 & 0,54 \\
\hline Anual & Cidade & 48 & Recife & Paulista \& Abreu e Lima & 6,25 & 95,86 & 0,52 \\
\hline Anual & Cidade & 50 & Recife & Paulista \& Olinda & 6,17 & 94,67 & 1,33 \\
\hline Anual & Cidade & 51 & Recife & Jaboatão dos Guararapes \& Cabo de Santo Agostinho & 6,09 & 93,5 & 0,7 \\
\hline Anual & Cidade & 52 & Recife \& Jaboatão dos Guararapes & Camaragibe & 6,09 & 35,89 & 0,49 \\
\hline Anual & Cidade & 55 & Recife & Olinda & 6,09 & 93,47 & 3,64 \\
\hline Mensal & $\begin{array}{l}\text { Família de } \\
\text { Produto }\end{array}$ & 4 & Vestuário \& Diversão & Joalherias & 2,92 & 15,17 & 0,75 \\
\hline Mensal & $\begin{array}{l}\text { Família de } \\
\text { Produto }\end{array}$ & 7 & Diversão & Serviços \& Postos de Gasolina & 2,89 & 36,1 & 0,83 \\
\hline Mensal & $\begin{array}{l}\text { Família de } \\
\text { Produto }\end{array}$ & 9 & Diversão & Serviços \& Alimentos & 2,83 & 35,33 & 0,58 \\
\hline Mensal & $\begin{array}{l}\text { Família de } \\
\text { Produto }\end{array}$ & 10 & Lojas em Geral \& Diversão & Livraria & 2,79 & 12,53 & 0,61 \\
\hline Mensal & $\begin{array}{l}\text { Família de } \\
\text { Produto }\end{array}$ & 13 & Diversão & Postos de Gasolina \& Alimentos & 2,77 & 34,65 & 1,03 \\
\hline Mensal & $\begin{array}{l}\text { Família de } \\
\text { Produto }\end{array}$ & 14 & Diversão & Lojas em Geral \& Livraria & 2,75 & 34,4 & 0,61 \\
\hline Mensal & $\begin{array}{l}\text { Famillia de } \\
\text { Produto }\end{array}$ & 19 & Vestuário \& Diversão & Perfumaria & 2,7 & 13,98 & 0,97 \\
\hline Mensal & $\begin{array}{l}\text { Família de } \\
\text { Produto }\end{array}$ & 21 & Lojas em Geral \& Diversão & Joalherias & 2,66 & 11,93 & 0,59 \\
\hline Mensal & $\begin{array}{l}\text { Familia de } \\
\text { Produto }\end{array}$ & 24 & Diversão & Livraria \& Farmácia & 2,64 & 33,01 & 0,63 \\
\hline Mensal & $\begin{array}{l}\text { Familia de } \\
\text { Produto }\end{array}$ & 28 & Diversão & Postos de Gasolina \& Lojas em Geral & 2,62 & 32,71 & 1,66 \\
\hline Mensal & $\begin{array}{l}\text { Família de } \\
\text { Produto }\end{array}$ & 29 & Diversão & Lojas em Geral \& Joalherias & 2,6 & 32,54 & 0,59 \\
\hline Mensal & $\begin{array}{l}\text { Família de } \\
\text { Produto }\end{array}$ & 31 & Postos de Gasolina \& Diversão & Veículos & 2,59 & 11,58 & 0,95 \\
\hline Mensal & $\begin{array}{l}\text { Família de } \\
\text { Produto }\end{array}$ & 34 & Diversão & Serviços \& Lojas em Geral & 2,59 & 32,35 & 0,97 \\
\hline Mensal & Família de & 37 & Diversão \& Alimentos & Serviços & 2,58 & 6,44 & 0,58 \\
\hline
\end{tabular}




\begin{tabular}{|c|c|c|c|c|c|c|c|}
\hline & Produto & & & & & & \\
\hline Mensal & $\begin{array}{l}\text { Família de } \\
\text { Produto }\end{array}$ & 39 & Lojas em Geral \& Diversão & Perfumaria & 2,58 & 11,58 & 0,8 \\
\hline Mensal & $\begin{array}{l}\text { Família de } \\
\text { Produto }\end{array}$ & 42 & Diversão & Lojas em Geral \& Alimentos & 2,56 & 32,05 & 1,1 \\
\hline Mensal & $\begin{array}{l}\text { Família de } \\
\text { Produto }\end{array}$ & 43 & Serviços \& Diversão & Alimentos & 2,56 & 5,37 & 0,58 \\
\hline Mensal & $\begin{array}{l}\text { Família de } \\
\text { Produto }\end{array}$ & 46 & Diversão & Telecomunicação / Eletrônicos \& Postos de Gasolina & 2,54 & 31,81 & 0,85 \\
\hline Mensal & $\begin{array}{l}\text { Família de } \\
\text { Produto }\end{array}$ & 49 & Diversão & Joalherias \& Farmácia & 2,53 & 31,59 & 0,6 \\
\hline Mensal & $\begin{array}{l}\text { Família de } \\
\text { Produto }\end{array}$ & 50 & Diversão & Viagens & 2,51 & 31,4 & 0,71 \\
\hline Mensal & $\begin{array}{l}\text { Família de } \\
\text { Produto }\end{array}$ & 53 & Diversão & Perfumaria \& Lojas em Geral & 2,51 & 31,38 & 0,8 \\
\hline Mensal & $\begin{array}{l}\text { Família de } \\
\text { Produto }\end{array}$ & 55 & Diversão & Vestuário \& Postos de Gasolina & 2,51 & 31,38 & 1,84 \\
\hline Mensal & $\begin{array}{l}\text { Família de } \\
\text { Produto }\end{array}$ & 59 & Diversão & Perfumaria \& Farmácia & 2,5 & 31,2 & 0,87 \\
\hline Mensal & $\begin{array}{l}\text { Família de } \\
\text { Produto }\end{array}$ & 61 & Farmácia \& Diversão & Livraria & 2,48 & 12,99 & 0,63 \\
\hline Mensal & $\begin{array}{l}\text { Família de } \\
\text { Produto }\end{array}$ & 63 & Vestuário \& Diversão & Livraria & 2,47 & 12,81 & 0,62 \\
\hline Mensal & $\begin{array}{l}\text { Família de } \\
\text { Produto }\end{array}$ & 68 & Diversão & Serviços \& Farmácia & 2,46 & 30,78 & 1,13 \\
\hline Mensal & $\begin{array}{l}\text { Família de } \\
\text { Produto }\end{array}$ & 70 & Diversão & Vestuário \& Alimentos & 2,45 & 30,66 & 1,23 \\
\hline Mensal & $\begin{array}{l}\text { Família de } \\
\text { Produto }\end{array}$ & 71 & Vestuário \& Diversão & Serviços & 2,44 & 12,68 & 1,15 \\
\hline Mensal & $\begin{array}{l}\text { Família de } \\
\text { Produto }\end{array}$ & 73 & Diversão & Vestuário \& Livraria & 2,44 & 30,53 & 0,62 \\
\hline Mensal & $\begin{array}{l}\text { Família de } \\
\text { Produto }\end{array}$ & 75 & Diversão & Postos de Gasolina \& Farmácia & 2,43 & 30,37 & 2,14 \\
\hline Mensal & $\begin{array}{l}\text { Família de } \\
\text { Produto }\end{array}$ & 81 & Diversão & Supermercado \& Livraria & 2,42 & 30,19 & 0,8 \\
\hline Mensal & $\begin{array}{l}\text { Família de } \\
\text { Produto }\end{array}$ & 85 & Diversão & Vestuário \& Serviços & 2,41 & 30,12 & 1,15 \\
\hline Mensal & $\begin{array}{l}\text { Família de } \\
\text { Produto }\end{array}$ & 87 & Diversão & Vestuário \& Joalherias & 2,4 & 30,04 & 0,75 \\
\hline Mensal & $\begin{array}{l}\text { Família de } \\
\text { Produto }\end{array}$ & 88 & Farmácia \& Diversão & Alimentos & 2,4 & 12,55 & 1,36 \\
\hline
\end{tabular}




\begin{tabular}{|c|c|c|c|c|c|c|c|}
\hline Mensal & Produto & 9 & Diversao_1_F03:19|--31 \& Diversao_1_F01:0|--12 & Diversao_1_F02:12|--19 & 11,82 & 8,76 & 0,33 \\
\hline Mensal & Produto & 29 & Diversao_1_F01:0|--12 & Diversao_1_F03:19|--31 \& Diversao_1_F02:12|--19 & 9,72 & 33,31 & 0,33 \\
\hline Mensal & Produto & 35 & Diversao_1_F01:0|--12 & Supermercado_1_F01:0|--13 \& Diversao_1_F02:12|--19 & 9,34 & 32,02 & 0,37 \\
\hline Mensal & Produto & 63 & Diversao_1_F01:0|--12 & Supermercado_1_F02:13|--28 \& Diversao_1_F02:12|--19 & 8,43 & 28,9 & 0,34 \\
\hline Mensal & Produto & 68 & Supermercado_1_F03:28|--61 \& Diversao_1_F01:0|--12 & Diversao_1_F02:12|--19 & 8,21 & 8,23 & 0,31 \\
\hline Mensal & Produto & 74 & Supermercado_1_F02:13|--28 \& Diversao_1_F01:0|--12 & Diversao_1_F02:12|--19 & 8,09 & 9,13 & 0,34 \\
\hline Mensal & Produto & 79 & Diversao_1_F01:0|--12 & Supermercado_1_F03:28|--61 \& Diversao_1_F02:12|--19 & 8,08 & 27,7 & 0,31 \\
\hline Mensal & Produto & 80 & Supermercado_1_F01:0|--13 \& Diversao_1_F01:0|--12 & Diversao_1_F02:12|--19 & 8,04 & 9,74 & 0,37 \\
\hline Mensal & Produto & 84 & Diversao_1_F02:12|--19 \& Diversao_1_F01:0|--12 & Diversao_1_F03:19|--31 & 7,92 & 7,2 & 0,33 \\
\hline Mensal & Produto & 119 & Diversao_1_F01:0|--12 & Diversao_1_F02:12|--19 & 7,06 & 24,2 & 0,91 \\
\hline Mensal & Produto & 129 & Diversao_1_F01:0|--12 & Supermercado_1_F01:0|--13 \& Diversao_1_F03:19|--31 & 6,92 & 23,71 & 0,28 \\
\hline Mensal & Produto & 157 & Diversao_1_F01:0|--12 & Supermercado_1_F02:13|--28 \& Diversao_1_F03:19|--31 & 6,18 & 21,18 & 0,28 \\
\hline Mensal & Produto & 167 & Diversao_1_F01:0|--12 & Supermercado_1_F03:28|--61 \& Diversao_1_F03:19|--31 & 6,04 & 20,71 & 0,27 \\
\hline Mensal & Produto & 175 & Supermercado_1_F03:28|--61 \& Diversao_1_F01:0|--12 & Diversao_1_F03:19|--31 & 5,82 & 5,84 & 0,27 \\
\hline Mensal & Produto & 198 & Supermercado_1_F02:13|--28 \& Diversao_1_F01:0|--12 & Diversao_1_F03:19|--31 & 5,45 & 6,15 & 0,28 \\
\hline Mensal & Produto & 214 & Supermercado_1_F01:0|--13 \& Diversao_1_F01:0|--12 & Diversao_1_F03:19|--31 & 5,14 & 6,23 & 0,28 \\
\hline Mensal & Produto & 249 & Diversao_1_F01:0|--12 & Diversao_1_F03:19|--31 & 4,73 & 16,21 & 0,74 \\
\hline Mensal & Produto & 362 & Diversao_1_F01:0|--12 & Supermercado_1_F01:0|--13 \& Farmacia_1_F01:0|--11 & 3,88 & 13,29 & 0,27 \\
\hline Mensal & Produto & 374 & Diversao_1_F01:0|--12 & Alimentos_1_F01:0|--12 & 3,78 & 12,94 & 0,38 \\
\hline Mensal & Produto & 392 & Diversao_1_F01:0|--12 & Diversao_1_F04:31|--45 & 3,72 & 12,75 & 0,32 \\
\hline Mensal & Produto & 450 & Diversao_1_F01:0|--12 & Supermercado_1_F02:13|--28 \& Farmacia_1_F01:0|--11 & 3,5 & 11,99 & 0,24 \\
\hline Mensal & Produto & 658 & Diversao_1_F01:0|--12 & Lojas em Geral_1_F01:0|--13 & 2,97 & 10,18 & 0,3 \\
\hline Mensal & Produto & 788 & Diversao_1_F01:0|--12 & Farmacia_1_F01:0|--11 & 2,79 & 9,55 & 0,56 \\
\hline Mensal & Produto & 820 & Diversao_1_F01:0|--12 & Alimentos_1_F02:12|--21 & 2,73 & 9,37 & 0,32 \\
\hline Mensal & Produto & 944 & Diversao_1_F01:0|--12 & Vestuario_1_F01:0|--15 & 2,53 & 8,68 & 0,23 \\
\hline Mensal & Produto & 967 & Diversao_1_F01:0|--12 & Supermercado_1_F02:13|--28 \& Supermercado_1_F01:0|--13 & 2,51 & 8,61 & 0,64 \\
\hline Mensal & Produto & 972 & Diversao_1_F01:0|--12 & Lojas em Geral_1_F02:13|--21 & 2,5 & 8,58 & 0,31 \\
\hline Mensal & Produto & 1006 & Diversao_1_F01:0|--12 & Supermercado_1_F03:28|--61 \& Supermercado_1_F01:0|--13 & 2,46 & 8,43 & 0,52 \\
\hline
\end{tabular}




\begin{tabular}{|c|c|c|c|c|c|c|c|}
\hline Mensal & Produto & 1043 & Diversao_1_F01:0|--12 & Postos de Gasolina_1_F01:0|--18 & 2,42 & 8,31 & 0,47 \\
\hline Mensal & Produto & 1173 & Diversao_1_F01:0|--12 & Supermercado_1_F04:61|--122 \& Supermercado_1_F01:0|--13 & 2,31 & 7,9 & 0,24 \\
\hline Mensal & Produto & 1265 & Diversao_1_F01:0|--12 & Supermercado_1_F01:0|--13 & 2,24 & 7,67 & 1,21 \\
\hline Mensal & Produto & 1320 & Diversao_1_F01:0|--12 & Vestuario_1_F02:15|--24 & 2,18 & 7,48 & 0,29 \\
\hline Mensal & Produto & 1337 & Diversao_1_F01:0|--12 & Farmacia_1_F02:11|--17 & 2,17 & 7,43 & 0,47 \\
\hline Mensal & Produto & 1399 & Diversao_1_F01:0|--12 & Postos de Gasolina_1_F02:18|--30 & 2,08 & 7,13 & 0,48 \\
\hline Mensal & Produto & 1406 & Diversao_1_F01:0|--12 & Lojas em Geral_1_F03:21|--37 & 2,07 & 7,11 & 0,26 \\
\hline Anual & $\begin{array}{l}\text { Família de } \\
\text { Produto }\end{array}$ & 1 & Veículos \& Diversão & Viagens & 2,75 & 31,28 & 2,61 \\
\hline Anual & $\begin{array}{l}\text { Família de } \\
\text { Produto }\end{array}$ & 4 & Diversão & Viagens \& Alimentos & 2,48 & 74,4 & 2,55 \\
\hline Anual & $\begin{array}{l}\text { Família de } \\
\text { Produto }\end{array}$ & 5 & Serviços \& Diversão & Viagens & 2,46 & 33,87 & 2,83 \\
\hline Anual & $\begin{array}{l}\text { Família de } \\
\text { Produto }\end{array}$ & 7 & Postos de Gasolina \& Diversão & Viagens & 2,45 & 42,24 & 3,53 \\
\hline Anual & $\begin{array}{l}\text { Familia de } \\
\text { Produto }\end{array}$ & 11 & Telecomunicação / Eletrônicos \& Diversão & Viagens & 2,41 & 38,21 & 3,19 \\
\hline Anual & $\begin{array}{l}\text { Família de } \\
\text { Produto }\end{array}$ & 14 & Diversão & Viagens \& Postos de Gasolina & 2,41 & 72,34 & 3,53 \\
\hline Anual & $\begin{array}{l}\text { Familia de } \\
\text { Produto }\end{array}$ & 16 & Diversão & Viagens \& Serviços & 2,4 & 72,23 & 2,83 \\
\hline Anual & $\begin{array}{l}\text { Família de } \\
\text { Produto }\end{array}$ & 26 & Diversão & Viagens \& Veículos & 2,37 & 71,25 & 2,61 \\
\hline Anual & $\begin{array}{l}\text { Família de } \\
\text { Produto }\end{array}$ & 29 & Joalherias \& Diversão & Perfumaria & 2,35 & 24,85 & 5,86 \\
\hline Anual & $\begin{array}{l}\text { Família de } \\
\text { Produto }\end{array}$ & 35 & Joalherias \& Diversão & Livraria & 2,32 & 24,53 & 4,74 \\
\hline Anual & $\begin{array}{l}\text { Família de } \\
\text { Produto }\end{array}$ & 45 & Diversão \& Alimentos & Viagens & 2,3 & 30,56 & 2,55 \\
\hline Anual & $\begin{array}{l}\text { Familia de } \\
\text { Produto }\end{array}$ & 52 & Diversão & Livraria \& Alimentos & 2,29 & 68,88 & 5,47 \\
\hline Anual & $\begin{array}{l}\text { Família de } \\
\text { Produto }\end{array}$ & 54 & Diversão & Viagens \& Telecomunicação / Eletrônicos & 2,29 & 68,87 & 3,19 \\
\hline Anual & $\begin{array}{l}\text { Família de } \\
\text { Produto }\end{array}$ & 55 & Geral \& Diversão & Livraria & 2,29 & 23,37 & 4,52 \\
\hline Anual & $\begin{array}{l}\text { Família de } \\
\text { Produto }\end{array}$ & 57 & Perfumaria \& Diversão & Livraria & 2,28 & 27,29 & 5,28 \\
\hline Anual & $\begin{array}{l}\text { Família de } \\
\text { Produto }\end{array}$ & 60 & Diversão & Viagens \& Farmácia & 2,28 & 68,48 & 4,22 \\
\hline
\end{tabular}




\begin{tabular}{|c|c|c|c|c|c|c|c|}
\hline Anual & $\begin{array}{l}\text { Família de } \\
\text { Produto }\end{array}$ & 63 & Perfumaria \& Diversão & Joalherias & 2,27 & 27,15 & 5,86 \\
\hline Anual & $\begin{array}{l}\text { Família de } \\
\text { Produto }\end{array}$ & 66 & Diversão & Joalherias \& Alimentos & 2,27 & 68,23 & 5,62 \\
\hline Anual & $\begin{array}{l}\text { Família de } \\
\text { Produto }\end{array}$ & 85 & Geral \& Diversão & Alimentos & 2,24 & 22,82 & 5,67 \\
\hline Anual & $\begin{array}{l}\text { Família de } \\
\text { Produto }\end{array}$ & 87 & Diversão \& Construção & Viagens & 2,24 & 32,89 & 2,75 \\
\hline Anual & Produto & 49 & Diversao_1_F05:45|--71 \& Diversao_1_F01:0|--12 & Diversao_1_F04:31|--45 & 6,72 & 16,86 & 1,72 \\
\hline Anual & Produto & 83 & Diversao_1_F04:31|--45 \& Diversao_1_F01:0|--12 & Diversao_1_F05:45|--71 & 6,37 & 24,69 & 1,72 \\
\hline Anual & Produto & 89 & Diversao_1_F01:0|--12 \& Alimentos_1_F02:12|--21 & Alimentos_1_F01:0|--12 & 6,36 & 20,85 & 1,99 \\
\hline Anual & Produto & 131 & Diversao_1_F05:45|--71\& Diversao_1_F01:0|--12 & Diversao_1_F02:12|--19 & 6,03 & 15,11 & 1,95 \\
\hline Anual & Produto & 191 & Diversao_1_F04:31|--45 \& Diversao_1_F01:0|--12 & Diversao_1_F02:12|--19 & 5,77 & 22,35 & 2,88 \\
\hline Anual & Produto & 237 & Diversao_1_F03:19|--31 \& Diversao_1_F01:0|--12 & Diversao_1_F02:12|--19 & 5,64 & 33,75 & 4,35 \\
\hline Anual & Produto & 298 & Diversao_1_F01:0|--12 & Diversao_1_F02:12|--19\& Alimentos_1_F01:0|--12 & 5,56 & 64,69 & 2,2 \\
\hline Anual & Produto & 389 & Diversao_1_F01:0|--12 \& Alimentos_1_F03:21|--39 & Alimentos_1_F01:0|--12 & 5,43 & 16,13 & 1,54 \\
\hline Anual & Produto & 450 & Diversao_1_F01:0|--12 & Lojas em Geral_1_F01:0|--13 \& Diversao_1_F02:12|--19 & 5,32 & 61,88 & 2,22 \\
\hline Anual & Produto & 468 & Diversao_1_F01:0|--12 & Servicos_1_F02:16|--30 \& Diversao_1_F02:12|--19 & 5,26 & 61,28 & 1,54 \\
\hline Anual & Produto & 501 & Diversao_1_F01:0|--12 \& Alimentos_1_F02:12|--21 & Diversao_1_F02:12|--19 & 5,21 & 17,08 & 2,2 \\
\hline Anual & Produto & 513 & Diversao_1_F01:0|--12 \& Alimentos_1_F01:0|--12 & Alimentos_1_F02:12|--21 & 5,2 & 17,42 & 1,99 \\
\hline Anual & Produto & 517 & Diversao_1_F01:0|--12 \& Alimentos_1_F03:21|--39 & Alimentos_1_F02:12|--21 & 5,19 & 15,43 & 1,77 \\
\hline Anual & Produto & 528 & Diversao_1_F01:0|--12 ～～～～ & Farmacia_1_F01:0|--11 \& Diversao_1_F02:12|--19 & 5,18 & 60,35 & 3,33 \\
\hline Anual & Produto & 553 & Servicos_1_F02:16|--30 \& Diversao_1_F01:0|--12 & Diversao_1_F02:12|--19 & 5,16 & 11,97 & 1,54 \\
\hline Anual & Produto & 569 & Diversao_1_F05:45|--71 \& Diversao_1_F01:0|--12 & Diversao_1_F03:19|--31 & 5,13 & 12,86 & 1,99 \\
\hline Anual & Produto & 578 & Diversao_1_F01:0|--12 & Supermercado_3_F01:0|--29 \& Diversao_1_F02:12|--19 & 5,13 & 59,69 & 1,57 \\
\hline Anual & Produto & 597 & Diversao_1_F01:0|--12 \& Alimentos_1_F01:0|--12 & Diversao_1_F02:12|--19 & 5,1 & 17,11 & 2,2 \\
\hline Anual & Produto & 602 & Diversao_1_F01:0|--12 & Vestuario_1_F01:0|--15 \& Diversao_1_F02:12|--19 & 5,1 & 59,38 & 1,94 \\
\hline Anual & Produto & 612 & Diversao_1_F01:0|--12 & Diversao_1_F05:45|--71 \& Diversao_1_F02:12|--19 & 5,09 & 59,27 & 1,95 \\
\hline Anual & Produto & 616 & Diversao_1_F01:0|--12 & Diversao_1_F02:12|--19 \& Alimentos_1_F02:12|--21 & 5,09 & 59,2 & 2,2 \\
\hline Anual & Produto & 631 & Diversao_1_F04:31|--45 \& Diversao_1_F01:0|--12 & Diversao_1_F03:19|--31 & 5,07 & 19,63 & 3,04 \\
\hline Anual & Produto & 661 & Diversao_1_F01:0|--12 \& Alimentos_1_F03:21|--39 & Diversao_1_F02:12|--19 & 5,05 & 14,99 & 1,93 \\
\hline
\end{tabular}




\begin{tabular}{|c|c|c|c|c|c|c|c|}
\hline Anual & Produto & 666 & Diversao_1_F01:0|--12 & Supermercado_3_F02:29|--44 \& Diversao_1_F02:12|--19 & 5,04 & 58,7 & 1,69 \\
\hline Anual & Produto & 671 & Lojas em Geral_1_F04:37|--81 \& Diversao_1_F01:0|--12 & Diversao_1_F02:12|--19 & 5,03 & 12,96 & 1,67 \\
\hline Anual & Produto & 693 & Postos de Gasolina_1_F03:30|--50 \& Diversao_1_F01:0|--12 & Diversao_1_F02:12|--19 & 5,01 & 20,53 & 2,65 \\
\hline Anual & Produto & 718 & Diversao_1_F01:0|--12 & Diversao_1_F03:19|--31 \& Diversao_1_F02:12|--19 & 4,99 & 58,14 & 4,35 \\
\hline Anual & Produto & 725 & Vestuario_2_F04:69|--104 \& Diversao_1_F01:0|--12 & Diversao_1_F02:12|--19 & 4,99 & 11,68 & 1,51 \\
\hline Anual & Produto & 743 & Diversao_1_F03:19|--31 \& Diversao_1_F01:0|--12 & Diversao_1_F04:31|--45 & 4,98 & 29,8 & 3,04 \\
\hline Anual & Produto & 755 & Postos de Gasolina_1_F02:18|--30 \& Diversao_1_F01:0|--12 & Diversao_1_F02:12|--19 & 4,97 & 20,27 & 2,61 \\
\hline Anual & Produto & 788 & Diversao_1_F01:0|--12 & Diversao_1_F03:19|--31 \& Alimentos_1_F01:0|--12 & 4,94 & 57,54 & 2,01 \\
\hline Anual & Produto & 796 & Diversao_1_F01:0|--12 & Diversao_1_F04:31|--45 \& Diversao_1_F02:12|--19 & 4,94 & 57,45 & 2,88 \\
\hline Anual & Produto & 802 & Diversao_1_F01:0|--12 & Vestuario_2_F04:69|--104 \& Diversao_1_F02:12|--19 & 4,92 & 57,33 & 1,51 \\
\hline Anual & Produto & 829 & Lojas em Geral_1_F01:0|--13 \& Diversao_1_F01:0|--12 & Diversao_1_F02:12|--19 & 4,9 & 17,22 & 2,22 \\
\hline Anual & Produto & 834 & Diversao_1_F01:0|--12 & Farmacia_1_F02:11|--17 \& Diversao_1_F02:12|--19 & 4,9 & 57,07 & 3,48 \\
\hline Anual & Produto & 847 & Postos de Gasolina_1_F01:0|--18 \& Diversao_1_F01:0|--12 & Diversao_1_F02:12|--19 & 4,89 & 18,53 & 2,39 \\
\hline Anual & Produto & 853 & Postos de Gasolina_1_F04:50|--62 \& Diversao_1_F01:0|--12 & Diversao_1_F02:12|--19 & 4,89 & 12,18 & 1,57 \\
\hline Anual & Produto & 877 & Lojas em Geral_1_F03:21|--37 \& Diversao_1_F01:0|--12 & Diversao_1_F02:12|--19 & 4,88 & 18,88 & 2,43 \\
\hline Anual & Produto & 891 & Vestuario_3_F03:82|--120 \& Diversao_1_F01:0|--12 & Diversao_1_F02:12|--19 & 4,87 & 13,34 & 1,72 \\
\hline Anual & Produto & 916 & Diversao_1_F01:0|--12 & Supermercado_3_F03:44|--80 \& Diversao_1_F02:12|--19 & 4,85 & 56,47 & 1,74 \\
\hline Anual & Produto & 932 & Diversao_1_F01:0|--12 & Postos de Gasolina_1_F01:0|--18 \& Diversao_1_F02:12|--19 & 4,84 & 56,4 & 2,39 \\
\hline Anual & Produto & 933 & Lojas em Geral_1_F02:13|--21 \& Diversao_1_F01:0|--12 & Diversao_1_F02:12|--19 & 4,84 & 19,31 & 2,49 \\
\hline Anual & Produto & 943 & Farmacia_1_F02:11|--17 \& Diversao_1_F01:0|--12 & Diversao_1_F02:12|--19 & 4,84 & 27,02 & 3,48 \\
\hline Anual & Produto & 962 & Diversao_1_F01:0|--12 & Lojas em Geral_1_F03:21|--37 \& Diversao_1_F02:12|--19 & 4,83 & 56,17 & 2,43 \\
\hline Anual & Produto & 969 & Vestuario_1_F02:15|--24 \& Diversao_1_F01:0|--12 & Diversao_1_F02:12|--19 & 4,82 & 19,22 & 2,48 \\
\hline Anual & Produto & 981 & Vestuario_1_F03:24|--38 \& Diversao_1_F01:0|--12 & Diversao_1_F02:12|--19 & 4,82 & 18,27 & 2,35 \\
\hline Anual & Produto & 989 & Vestuario_1_F04:38|--68 \& Diversao_1_F01:0|--12 & Diversao_1_F02:12|--19 & 4,82 & 12,47 & 1,61 \\
\hline Anual & Produto & 1001 & Vestuario_2_F02:35|--49 \& Diversao_1_F01:0|--12 & Diversao_1_F02:12|--19 & 4,81 & 14,99 & 1,93 \\
\hline Anual & Produto & 1005 & Supermercado_3_F01:0|--29 \& Diversao_1_F01:0|--12 & Diversao_1_F02:12|--19 & 4,81 & 12,15 & 1,57 \\
\hline Anual & Produto & 1008 & Diversao_1_F01:0|--12 & Vestuario_1_F02:15|--24 \& Diversao_1_F02:12|--19 & 4,81 & 56 & 2,48 \\
\hline Anual & Produto & 1009 & Supermercado_3_F02:29|--44 \& Diversao_1_F01:0|--12 & Diversao_1_F02:12|--19 & 4,81 & 13,11 & 1,69 \\
\hline Anual & Produto & 1018 & Diversao_1_F01:0|--12 & Vestuario_1_F04:38|--68 \& Diversao_1_F02:12|--19 & 4,8 & 55,92 & 1,61 \\
\hline
\end{tabular}




\begin{tabular}{|c|c|c|c|c|c|c|c|}
\hline Anual & Produto & 1019 & Farmacia_1_F03:17|--27 \& Diversao_1_F01:0|--12 & Diversao_1_F02:12|--19 & 4,8 & 25,43 & 3,28 \\
\hline Anual & Produto & 1023 & Vestuario_1_F01:0|--15 \& Diversao_1_F01:0|--12 & Diversao_1_F02:12|--19 & 4,8 & 15,05 & 1,94 \\
\hline Anual & Produto & 1027 & Farmacia_1_F04:27|--44 \& Diversao_1_F01:0|--12 & Diversao_1_F02:12|--19 & 4,79 & 16,27 & 2,1 \\
\hline Anual & Produto & 1034 & Diversao_1_F01:0|--12 & Lojas em Geral_1_F02:13|--21 \& Diversao_1_F02:12|--19 & 4,79 & 55,78 & 2,49 \\
\hline
\end{tabular}

Tabela 48: Regras de associação das AAs utilizadas na segmentação dos clientes 